\title{
Structure of charged two-component lipid membranes and their interaction with colloids studied by different X-ray and microscopy techniques
}

\author{
Dissertation \\ zur Erlangung des Doktorgrades \\ der Mathematisch-Naturwissenschaftlichen Fakultäten \\ der Georg-August-Universität zu Göttingen \\ vorgelegt von \\ Eva Nováková \\ aus Ružomberok, Slowakei
}

Göttingen 2008 
D7

Referent: Prof. Dr. Tim Salditt

Koreferent: Prof. Dr. Christoph F. Schmidt

Tag der mündlichen Prüfung: 9. Juli 2008 




\section{Contents}

$\begin{array}{lll}\text { Chapter 1. Introduction } & 1\end{array}$

Chapter 2. Biomembranes and Colloids 5

2.1. Biomembranes 5

$\begin{array}{lll}2.1 .1 . & \text { Membrane structure and properties } & 6\end{array}$

$\begin{array}{lll}2.1 .2 . & \text { Phase transition } & 6\end{array}$

$\begin{array}{lll}2.2 . & \text { Charged membranes } & 7\end{array}$

2.2.1. Diffuse double layer - Gouy-Chapman theory 9

\begin{tabular}{lll}
\hline 2.2 .2$. & Elastic properties of fluid-like membranes & 13
\end{tabular}

$\begin{array}{lll}2.3 . & \text { Solid supported biomimetic lipid bilayers } & 13\end{array}$

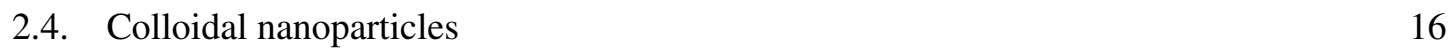

$\begin{array}{ll}\text { 2.4.1. Colloid-membrane interaction } & 17\end{array}$

$\begin{array}{lll}\text { Chapter 3. } & \text { Materials and Preparation Techniques } & 19\end{array}$

$\begin{array}{ll}\text { 3.1. Small unillamelar vesicles } & 19\end{array}$

3.2. Two-component solid supported lipid membranes 21

\begin{tabular}{|lll}
3.3. & Suppported lipid membrane mixed with amidine latex colloids & 24
\end{tabular}

3.4. Suppported lipid membrane mixed with gold colloids 25

3.5. Giant unilamellar vesicles mixed with gold colloids 26

\begin{tabular}{lll}
\hline 3.6. & Lipid multilayers & 27
\end{tabular}

Chapter 4. Introduction to X-ray interaction with matter, index of refraction 29

$\begin{array}{lll}\text { Chapter 5. X-ray reflectivity study of solid supported lipid membranes } & 33\end{array}$

$\begin{array}{lll}5.1 . & \text { Introduction } & 33\end{array}$

5.2. X-ray reflectivity: theory, experiment, and analysis 34

\begin{tabular}{|ll}
$5.2 .1 . \quad$ Critical angle of reflection & 34
\end{tabular}

\begin{tabular}{|lll}
5.2 .1 .1$. & Reflected intensity from ideally flat surface & 35
\end{tabular}

$\begin{array}{lll}5.2 .2 . & \text { Kinematical theory } & 36\end{array}$

$\begin{array}{lll}5.2 .3 . & \text { Rough surfaces and interfaces } & 37\end{array}$

\begin{tabular}{|lll}
\hline 5.2 .4$. & X-ray reflectivity from a single lipid membrane & 37
\end{tabular}

$\begin{array}{lll}5.2 .5 . & \text { Experimental Setup } & 38\end{array}$

$\begin{array}{lll}5.2 .5 .1 . & \text { X-ray reflectivity measurement } & 39\end{array}$

$\begin{array}{lll}5.2 .6 . & \text { Radiation damage and reproducibility } & 41\end{array}$

5.2.7. Data Treatment 42

5.3. Semi-kinematical model for lipid bilayer reflectivity 44 
$\begin{array}{lll}\text { 5.4. Results and Discussion } & 48\end{array}$

5.4.1. Mixed bilayer in the fluid phase $\quad 49$

$\begin{array}{lll}5.4 .1 .1 . \quad \mathrm{DOPC} & 49\end{array}$

$\begin{array}{lll}5.4 .1 .2 . & \text { DOPC/DOPS(4:1) } & 49\end{array}$

$\begin{array}{lll}5.4 .1 .3 . & \text { DOPC/DOTAP(9:1) } & 51\end{array}$

5.4.2. Mixed bilayer in the gel phase and lateral demixing 52

$\begin{array}{lll}5.4 .2 .1 . & \text { DPPC/DPPS(4:1) } & 52\end{array}$

$\begin{array}{lll}5.4 .2 .2 . & \text { DPPC/DPPS(1:1) } & 52\end{array}$

5.4.3. Reflectivity measurements of the dry silicon substrate 54

$\begin{array}{lll}5.5 . & \text { Summary and Conclusions } & 55\end{array}$

Chapter 6. X-ray spectro-microscopy study of solid supported two-component lipid membranes mixed with charged colloids $\quad 57$

$\begin{array}{ll}6.1 . \text { Introduction } & 57\end{array}$

$\begin{array}{lll}6.2 . & \text { Near-edge X-ray absorption fine structure } & 58\end{array}$

$\begin{array}{lll}6.2 .1 . & \text { X-ray absorption cross-section } & 59\end{array}$

$\begin{array}{lll}6.2 .2 . & \text { Transition energies } & 60\end{array}$

6.3. NEXAFS contrast soft X-ray spectromicroscopy 62

$\begin{array}{lll}6.4 . & \text { Instrumentation and data acquisition } & 63\end{array}$

\begin{tabular}{|lll}
6.4 .1$. & Scanning transmission X-ray microscope (STXM) at BESSY II & 63
\end{tabular}

$\begin{array}{lll}6.4 .2 . & \text { Laboratory scale XUV source } & 64\end{array}$

$\begin{array}{lll}6.5 . & \text { Results } & 65\end{array}$

6.5.1. Elemental Contrast Imaging 65

\begin{tabular}{|lll}
6.5 .2$. & Spectromicroscopy of dried amidine functionalized polystyrene particles & 67
\end{tabular}

\begin{tabular}{|lll}
6.5 .3$. & Spectromicroscopy of the lipid layer with polystyrene microspheres & 68
\end{tabular}

$\begin{array}{lll}6.5 .4 . \quad \text { NEXAFS spectra of phospholipid multilayers } & 71\end{array}$

\begin{tabular}{lll}
\hline 6.6. & Summary and Conclusion & 73
\end{tabular}

$\begin{array}{lll}\text { Chapter 7. } & \text { Interaction of two-component anionic lipid membrane with gold particles } & 77\end{array}$

$\begin{array}{ll}7.1 . & \text { Introduction } \\ & 77\end{array}$

$\begin{array}{lll}\text { 7.2. } & \text { Characterization of PSS/PAH coated gold particles } & 78\end{array}$

$\begin{array}{lll}7.3 . & \text { Dissociation constant of phosphatidylserine } & 78\end{array}$

7.4. $\quad$ Supported DOPC:DOPC(4:1) in fluid phase interacting with gold particles $\quad 83$

$\begin{array}{lll}7.5 . & \text { Supported DPPC:DPPC(4:1) in gel phase interacting with gold particles } & 86\end{array}$

$\begin{array}{lll}7.6 . & \text { DOPC:DOPC(4:1) GUV interacting with gold particles } & 86\end{array}$

$\begin{array}{lll}\text { 7.7. } & \text { Electrostatic interactions between membrane and gold particles } & 89\end{array}$

$\begin{array}{lll}7.8 . & \text { Conclusion } & 93\end{array}$

\begin{tabular}{lll}
\hline Chapter 8. Summary & 95
\end{tabular}

\begin{tabular}{lll}
\hline Appendix A. Fitting parameters & 97
\end{tabular}

\begin{tabular}{|ll}
\hline Appendix B. Igor Pro procedures & 99
\end{tabular} 
\begin{tabular}{lll}
\hline Appendix C. & Program listing & 103
\end{tabular}

\begin{tabular}{lr}
\hline List of abbreviations & 109
\end{tabular}

\begin{tabular}{ll}
\hline Bibliography & 111
\end{tabular}

\begin{tabular}{ll}
\hline Acknowledgements & 119
\end{tabular}

\begin{tabular}{ll}
\hline List of publications & 121
\end{tabular}

\begin{tabular}{lc}
\hline Lebenslauf & 123
\end{tabular} 



\section{CHAPTER 1}

\section{Introduction}

All cells are surrounded by a plasma membrane; eukaryotic cells also have membrane-bound organelles such as nuclei, endoplasmatic reticulum, Golgi complexes, lysosomes and mitochondria. A biological membrane is a complex aggregate of lipids, carbohydrates and proteins, formed as a result of noncovalent interactions. Such aggregates consist of hydrophobic regions separated from aqueous phases by interfaces [1]. Membranes not only define the boundaries of cells and divide the volume into discrete functional compartments but also regulate molecular traffic across boundaries, as well as other communications between cells and organelles [2]. Membranes have remarkable physical properties of being flexible, self-sealing, and selectively permeable to specific polar solutes [3]. Their flexibility permits changes in shapes essential to cell expansion or contraction. Fluid membranes can vary their topology; for instance in exocytosis, endocytosis and cell division. The selective permeability of membranes guarantees that certain compounds and ions can be retained within cells or specific membrane-enclosed organelles while other species are excluded [1]. In almost all of the above biological functions, membranes interact with other bodies, in the most general sense colloidal particles, regardless of whether the interaction partner is a protein network or a lipid structure such as a vesicle.

The aim of this thesis is to study the structure of charged lipid membranes and their interaction with oppositely charged colloidal particles.

Electrostatic interactions seem to be one of the most important forces in determining the way macromolecules interact with membranes. Charged lipids are common and versatile constituents of a biological membrane. Protein trafficking, adsorption and membrane insertion involve electrostatic interactions at some stage [4, 5, 6]. Membrane adsorption of small peptides like melittin [7] depends on the lipid bilayer's surface charge. Charged lipids participate in fusion events [8] , endocytosis [9], exocytosis [10]. The amount of charge on the surface of the lipid bilayer affects ion channel conductivity [11, 12].

Solid supported biomimetic lipid bilayers serve as simple model systems for the much more complex biological counterparts [13]. Supported lipid bilayers are single unilamellar lipid membranes, supported on a hydrophilic solid substrate, such as quartz, glass, or oxidized silicon. Planar bilayer systems are amenable to quantitative high-resolution analytical techniques, which can not (or not yet) be applied to real biological membranes.

Colloidal nanoparticles exhibit many functional properties, whose nature may be electronic, chemical, biological, mechanical, etc. Quantitative understanding of interactions between charged colloids and biological interfaces, in particular the cell membrane, is a prerequisite for the design 
of drug delivery systems based on synthetic nanoscale carrier systems, as well as for imaging agents such as fluorescent quantum dot markers. Functionally relevant issues are related to the binding affinity, the range of interaction, possible toxic effects due to membrane disruption or lysis [14, 15, 16], as well as the lateral reorganization of a multi-component membrane in response to nanoparticle binding [17].

In this thesis we study multi-component membrane model systems on solid support, from a structural point of view, mainly using X-ray techniques. X-rays were discovered by W.C. Röntgen in 1895. Since that time they have become an invaluable probe of the structure of matter. X-rays based techniques allow to probe structural features of surfaces and bulk on the length scales of a few $\AA$ and below. Specular X-ray reflectivity using synchrotron radiation is a powerful method to determine the structure of thin organic films by obtaining a one dimensional electron density profile along the surface normal.

The unique properties of $\mathrm{X}$-rays make them an attractive not only for diffraction but also for microscopy techniques. For example, soft X-ray microscopes have established capabilities in absorption contrast imaging of thick hydrated biological material in near-native environments at spatial resolutions well beyond those achievable with visible light microscopy. In near-edge $\mathrm{X}$-ray absorption spectro-microscopy, inner-shell electronic excitations are used as a chemically sensitive image-contrast mechanism [18]. In the energy range of the so called "water window" between oxygen and the carbon $K$ absorption edges $(283 \mathrm{eV}$ and $543 \mathrm{eV})$, specimens in water show a natural absorption contrast, which permits imaging of unstained specimens in a hydrated state.

In the first part of this thesis X-ray reflectivity is used to probe the structure of solid supported two-component lipid mixtures, with varied surface charges, both in the fluid and the gel phase. Structural parameters, such as membrane thickness, area per lipid, water thickness, and the number of water molecules per lipid, can be calculated directly from an electron density profile of the model bilayers. The resolution obtained in the density profile across the bilayer is high enough to distinguish two head-group maxima in the profile if the sample is in the phase coexistence regime. Another, more technical motivation for the present study is to demonstrate feasibility of single membrane reflectivity experiments with sealed tube in-house instrumentation.

In the second part, synchrotron-based scanning transmission soft X-ray spectro-microscopy is applied to address the lateral organization of two-component lipid membranes with addition of colloidal particles. Single bilayer patches with bound polystyrene microspheres, coated by a charged monolayer are imaged. The ability and current limits of scanning transmission X-ray spectro-microscopy to examine samples under physiologically relevant conditions in the presence of excess water is tested. We obtain reference spectra based on a compact laser driven plasma source, while the spectro-microscopy data are collected using synchrotron radiation at a lateral resolution of about $60 \mathrm{~nm}$. The main motivation is to show that single bilayer sensitivity can indeed be reached under physiological conditions, and that membrane colloid interaction as well as eventual lateral segregation of lipid components may be probed in future by this technique. 
The aim of the third part is to study how charged membranes response to oppositely charged polyelectrolyte-coated gold nanoparticles. We use fluorescence microscopy and X-ray reflectivity methods to probe the interactions between polyelectrolyte coated gold nanoparticles with supported lipid membranes and giant unilamellar vesicles. The motivation of this study is to demonstrate the importance of the $\mathrm{pH}$, ionic strength and membrane phase as a modulating parameter of the interaction.

This thesis is organized as follows. Chapter 2 addresses the biological and physical background of charged membranes and colloids, as well an overview of supported lipid membrane systems. In Chapter 3 , preparation techniques and materials used in this study are described. Chapter 4 presents a brief introduction to X-ray interaction with materials and derivation of the index of refraction. Chapter 5, 6 and 7, respectively, present the original research of this thesis: X-ray reflectivity study of solid supported lipid membranes, X-ray spectro-microscopy study of solid supported lipid membranes mixed with charged particles, and finally a study of interactions of two-component anionic lipid membranes with gold particles. Chapter 8 summarizes our conclusions and observations. 



\section{CHAPTER 2}

\section{Biomembranes and Colloids}

\subsection{Biomembranes}

The first comprehensive model of the biological membrane was presented by Singer and Nicolson, and is commonly referred to as 'the fluid mosaic model' [19]. According to this model, the biological membrane consists of a dynamic fluid like layer of phospholipids in which are embedded proteins that carry out all biologically relevant functions of membranes requiring specificity or selectivity. The 'fluid mosaic model' assumes the lipid bilayer to be a passive, uncomplicated structure fulfilling two basic functions: supporting proteins and forming a barrier for dissolved molecules in the aqueous phase. The structure and global behavior of model lipid bilayers were sufficiently described, within this model, by properly chosen macroscopic parameters such as fluidity, surface charge density, polarity, surface pressure, etc. [1, 20].

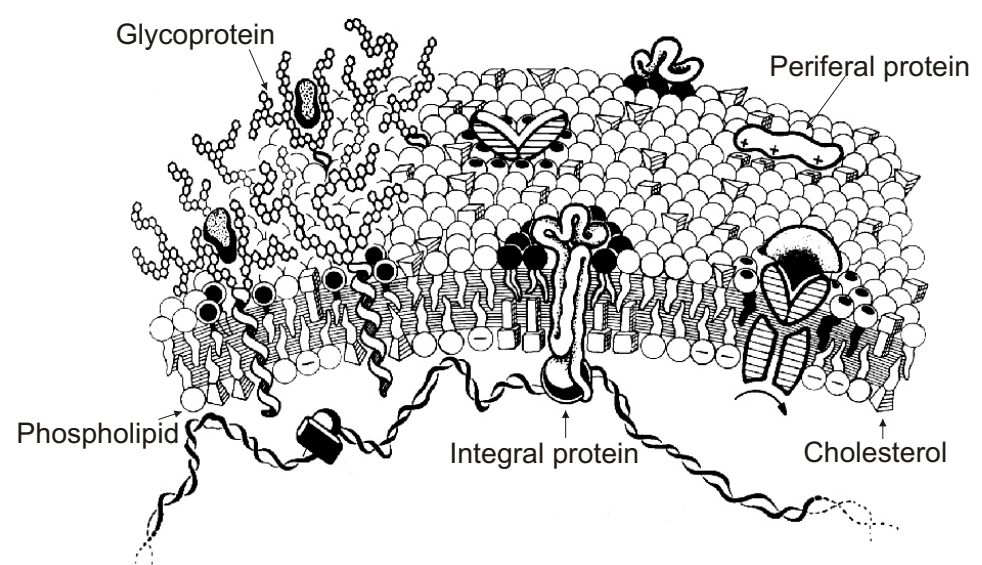

FIGURE 2.1.1. The schematic illustration of the plasma membrane of a cell [21]. The lipid membrane is the main structural element in which proteins are embedded.

By experimental data obtained in studies on biological and model membrane systems the original 'fluid mosaic model' was improved with the addition of some significant structural modifications, such as transversal asymmetry and the lateral non-uniform distribution of membrane components. Lipids were thus no longer assumed to be passive membrane components, but the lipid bilayer itself was recognized as a potent enhancer and regulator of surface associated reactions [22, 23] 


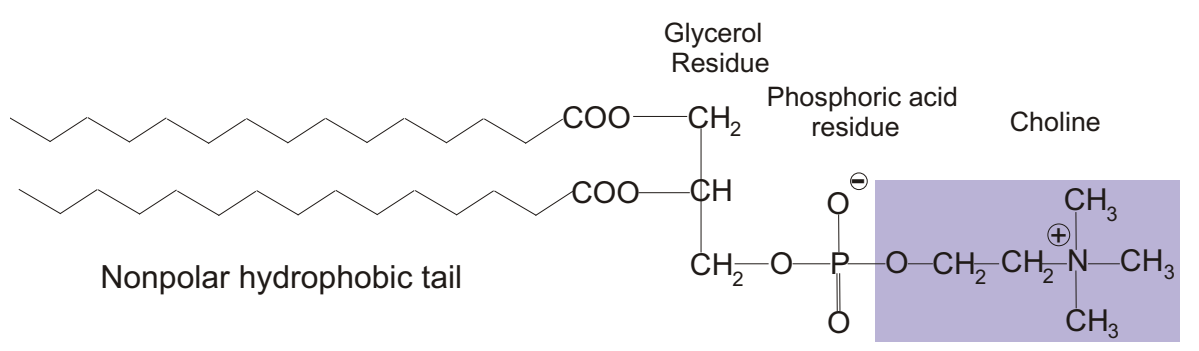

Polar hydrophilic head group

FIGURE 2.1.2. Chemical structure of a phospholipid 1,2 Dipalmitoyl-snGlycero-3-Phosphocholine, DPPC.

2.1.1. Membrane structure and properties. Lipids are surfactants with a hydrophilic headgroup and two hydrophobic chains. Lipid chains vary both in length and in saturation (number of double bonds). In the presence of water, lipids self-assemble into bilayers.

The term lipid comprises a diverse range of molecules such as fatty-acid derived phospholipids, sphingolipids, glycolipids and terpenoids, such as retinoids and steroids. Lipids are amphiphilic molecules since they consist of polar, hydrophilic headgroups and nonpolar, hydrophobic tails. This amphiphilic nature of lipids is responsible for their self assembly properties. The hydrophobic tails tend to be packed together away from the water surrounding while lipid heads face towards it. Therefore, lipids will aggregate in aqueous environment if a certain lipid concentration called critical micelle concentration is exceeded. Among a variety of structures lipids may form micelles, vesicles or bilayer structures depending on the exact size relation of the hydrophobic to the hydrophilic part. Cone-shaped molecules tend to form micelles while cylindrical molecules prefer to form bilayers. A bilayer consists of two monolayers of lipids where the hydrophobic tails face each other and the hydrophilic heads shield them from the surrounding water. This structure is also called a membrane and is the fundamental structure of biomembranes.

Phospholipids or, more precisely, glycerophospholipids, are built on a glycerol core to which two fatty acid-derived "tails" are linked by ester linkages and one "head" group by a phosphate ester linkage. Fatty acids are unbranched hydrocarbon chains, connected by single bonds alone (saturated fatty acids) or by both single and double bonds (unsaturated fatty acids). The chains are usually 10-24 carbon groups long. Common fatty acid chain residues are myristol which has 14 carbon groups and no double bond (14:0), palmitoyl (16:0), and oleoyl (18:1). Typical head groups of phospholipids found in biological membranes are phosphatidylcholine (lecithin), phosphatidylethanolamine, phosphatidylserine and phosphatidylinositol.

2.1.2. Phase transition. A lipid bilayer undergoes multiple phase transitions upon heating. The phase transition temperature, $T_{m}$, required to induce a change in the lipid physical state from the ordered gel phase to the disordered liquid crystalline phase. Below the chain melting temperature one-component bilayers are in the gel or solid phase where the acyl chains are stretched in an all-trans configuration and the lateral mobility is low. Above $T_{m}$ the lipids are in the fluid or liquid-crystalline phase which is characterized by high lateral mobility and disordered acyl chains. 
At low temperatures, a highly ordered sub-gel $\left(L_{c}\right)$ phase may exist; heating leads to the formation of a gel $\left(L_{\beta}\right)$ phase, which may be followed by an intermediate ripple $\left(P_{\beta^{\prime}}\right)$ phase, and finally a liquid-crystalline $\left(L_{\alpha}\right)$ phase [24] (Fig. 2.1.3). There are several factors which directly affect the phase transition temperature including hydrocarbon chain length, unsaturation, charge, and headgroup species [25].
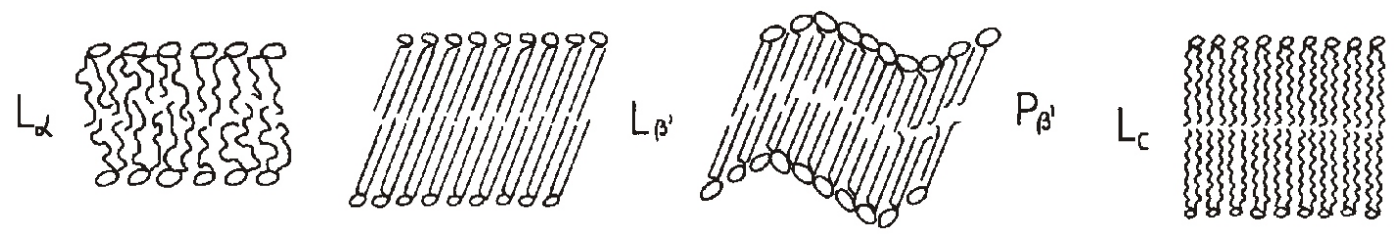

FIGURE 2.1.3. Schematic representation some of possible phases that occur in phospholipid bilayers: fluid $\left(L_{\alpha}\right)$ phase, tilted gel $\left(L_{\beta^{\prime}}\right)$ phase, ripple $\left(P_{\beta^{\prime}}\right)$ phase and sub-gel $\left(L_{c}\right)$ phase.

\subsection{Charged membranes}

Many biological membranes carry a net negative charge on their surface. Phosphatidylcholine (PC), sphingomyelin (SM) and phosphatidylethanolamine (PE) are the most abundant lipids in biological membranes, holding both negative and positive charge (such lipids are zwitterions). Headgroups of other lipids may carry negative net electrostatic charge (phosphatidylserine (PS), phosphatidic acid (PA), phosphatidylglycerol (PG)), positive charge (sphingosine and artificial cationic lipids) or lack charged residues (diacylglycerol and cerebroside) [23].

The major naturally occurring anionic phospholipids are phosphatidylserine, phosphatidylinositol (PI), phosphatidic acid and cardiolipin. Some bacterial systems also contain phosphatidylglycerol. Positively charged lipids are not found in the nature. They can, however, be chemically synthesized for gene delivery purposes and for studies on the interactions between DNA and membranes [26, 27]. For example, rat liver cells have plasma membranes consisting of PE in 23\%, PC in $39 \%$, PI in $8 \%$, PS in $9 \%$, SM in $16 \%$, a substantial amount of cholesterol and a small fraction of other lipids [1]. Some examples of lipids with various charged residue arrangements within their headgroups are shown in Fig. 2.2.1.

Choline and ethanolamine surfaces are different in many respects. The most relevant is a result of the hydration of both headgroups. Choline methyl group is hydrophobic, and adjacent water molecules are hydrogen bonded between themselves, forming a clathrate shell around the PC headgroup. It is estimated that about 25-30 water molecules are needed to fully hydrate the choline headgroup, whereas ethanolamine requires only 10-12 water molecules [28]. The differences between PE and PC surfaces affect interactions between macromolecules and respective membranes.

Phosphatidylserine has three residual charges: two are negative and associated with the phosphate and carboxyl groups, while the remaining positive charge is contained in the ammonium group. The resulting net charge is negative. Molecular dynamic simulation shows that PS membrane shows some differences in its behavior relative to uncharged phospholipids, in particular 


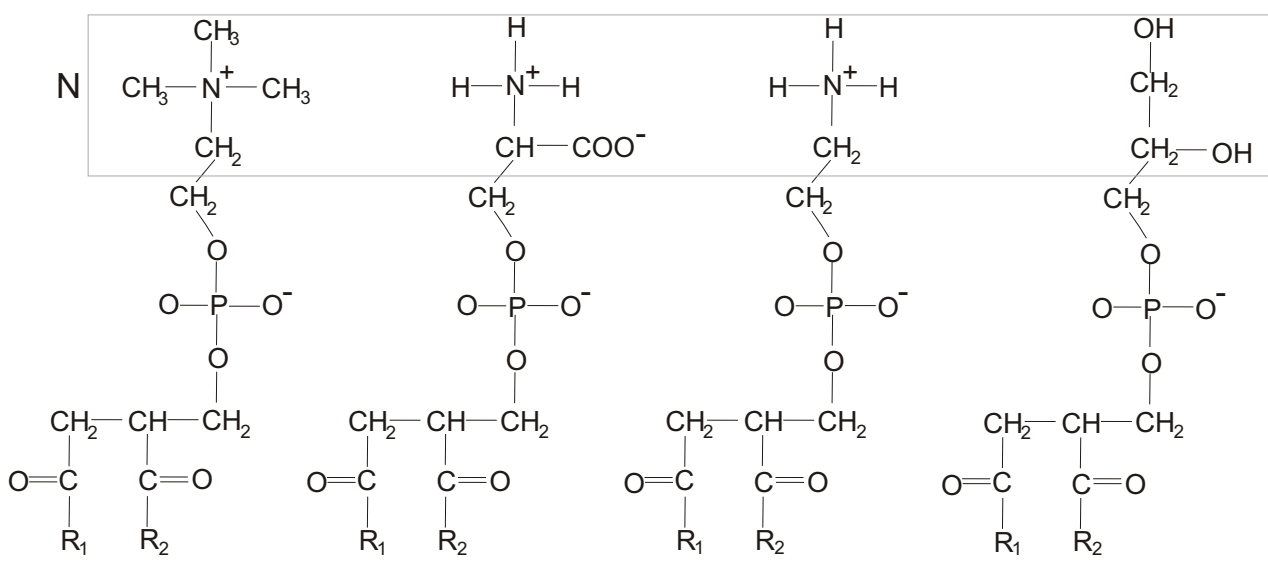

Phosphatidylcholine Phosphatidylserine Phosphatidylethanolamine Phosphatidylglycerol

FIGURE 2.2.1. Examples of some lipids with different charged residue arrangements within their headgroups: phosphatidylcholine, phosphatidylserine, phosphatidylethanolamine and phosphatidylglycerol. Negatively charged phosphate is a common feature of all presented molecules. The major difference between phospholipids is in the $\mathrm{N}$ region, where charges vary in quantity and size [23].

PC. These differences are mainly caused by charge interactions between adjacent phospholipids. These charge interactions are so effective that they are able to compensate the high electrostatic repulsions between neighboring phospholipids. They reduce the net surface area per phospholipid around $10 \%$ compared to PC. PS more closely resembles a model of two charged planes in a condensor. The local charge density is only partially compensated by water and causes an appreciable potential difference over the interface, with the water phase being positive with respect to the membrane interior [29, 30].

The intracellular side of the plasma membrane differs from the outer in the lipid composition, structure and function [23]. The presence of charged groups at the interface implies that electrostatic interactions are frequent in this region. In a living cell, the majority of PS is localized at the inner, cytosolic side of the plasma membrane; when located at the outer leaflet, PS is indicative of apoptosis [31]. Because of its net negative charge at physiological $\mathrm{pH}$, it may play an important role in biomembrane functioning as the properties of this negatively charged phospholipid are largely dependent on environmental conditions. The interaction of PS with monovalent and divalent cations may be involved in membrane-associated processes such as lipid phase modulation or separation, fusion events, and enzyme regulation [23]. In natural membranes, PS invariably coexists with zwitterionic phospholipids, namely, phosphatidylcholine.

Protonation and deprotonation of the phospholipid headgroups change the chemical nature and net charge of the lipid bilayers. It also may affect the molecular conformation the hydrogen bonding capabilities of individual lipid molecules and may modify the colloidal properties of the lipid aggregates, phospholipid phase transition, lipid bilayer interaction with protein molecules, etc. [20]. Proton binding can be described in terms of the logarithm of proton binding constant, 
$K_{H}$. One should distinguish between the apparent $p K_{a H}$ and the intrinsic $p K_{H}$ proton binding constants. The apparent $p K_{a H}$ for a single site group is determined as the bulk $\mathrm{pH}$ at which $50 \%$ of the group population is charged. The intrinsic $p K_{H}$ is determined as the surface $\mathrm{pH}$ at which 50 $\%$ of the group population is charged. The relation between these quantities is simply

$$
p K_{a H}=p K_{H}-0.434 \frac{e \psi_{S}}{k_{B} T}
$$

where $e$ is the elementary charge, $\psi_{S}$ is the electrostatic surface potential, and $k_{B}$ and $T$ have their usual meanings. The apparent $p K_{a H}$ is a directly measurable quantity, but depends on the experimental conditions such as the ionic strength of the medium and the amount of charged lipids in the membrane. The intrinsic $p K_{H}$, on the other hand, is a fundamental property of the system and is the most useful quantity. Negatively charged membranes became neutral upon protonation. The stepwise protonation of phosphatidylcholine and phosphatidylserine lipids is depicted in Fig. 2.2 .2 ,

The $p K_{a H}$ of a charged membrane is strongly influenced by ionic strength. The lower the ionic strength, the higher the $p K_{a H}[\mathbf{2 0}]$, as can be seen from the Eq. 7.3.2 by counterions screening of the negative potential $\psi_{S}$.

2.2.1. Diffuse double layer - Gouy-Chapman theory. The interplay between charged membranes and their surrounding ionic solution can simply be explained as the following. As any charged object immersed in an ionic solution, the membrane attracts a cloud of opposite charges forming a diffusive electric double layer [32, 33, 34]. The exact distribution of the charges is given by the competition between the electrostatic interactions and the entropy of the ions in the solution which tends to disperse them. This diffusive electric double layer in turn influences the overall electrostatic interactions of the membrane with its environment as well as the internal membrane properties [35].

The characterization of the electrostatic potential at the headgroups is limited to solution of the Poisson-Boltzmann equation (mean field theory). We consider a negatively charged singlesided flat membrane. The membrane surface occupies the $z=0$ plane, and has a constant surface charge density $\sigma$ (see Fig. 2.2.3). The aqueous solution occupies the positive half space, $z>0$. The electric field vanishes for large $z$ and is taken as zero for $z<0^{1}$. The surface charge of the interface is represented by the charged headgroups of the membrane phospholipid molecules.

The ionic solution contains, in general, both anions and cations, and is characterized by a dielectric constant $\varepsilon_{W}$ of the water throughout the fluid. The solution is in contact with an electrolyte (salt) reservoir of fixed concentration $n_{0}$. Two types of charge carriers are present in the solution: co-ions and counterions and both types are in thermal equilibrium with the reservoir. We assume for simplicity only one type of co-ions and one of counterions. The total charge density $\rho(\vec{r})$ at

\footnotetext{
${ }^{1}$ The simplifying assumption that the electric field does not penetrate inside the 'oily' part of the membrane, namely, where the aliphatic 'tails' are packed, can be justified [36] for typical values of membrane thickness and $\varepsilon_{\text {oil }} / \varepsilon_{W}$, where $\varepsilon_{o i l}$ and $\varepsilon_{W}$ are hydrocarbon and water dielectric constants, respectively. It is valid as long as the ratio of the two dielectric constants, $\varepsilon_{\text {oil }} / \varepsilon_{W}$, is much smaller than the ratio $t / \lambda_{D}$, where $t$ is the membrane thickness and $\lambda_{D}$ is the Debye-Hückel screening length, which will be defined later in this chapter [35].
} 


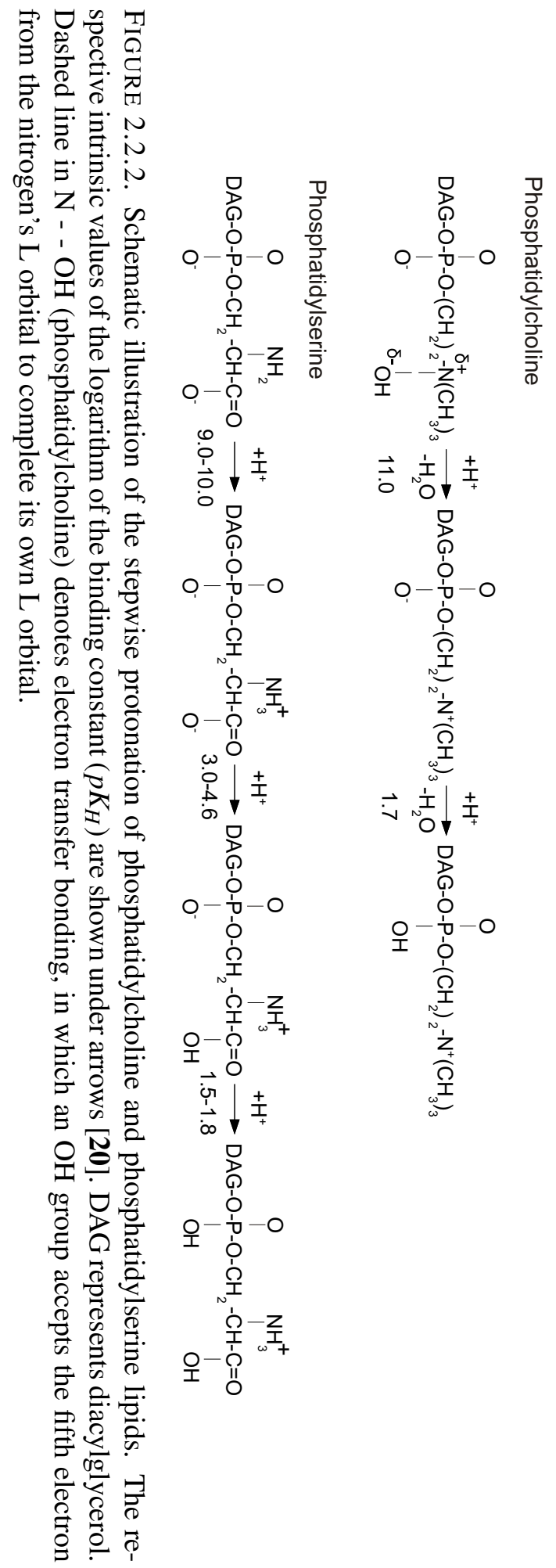




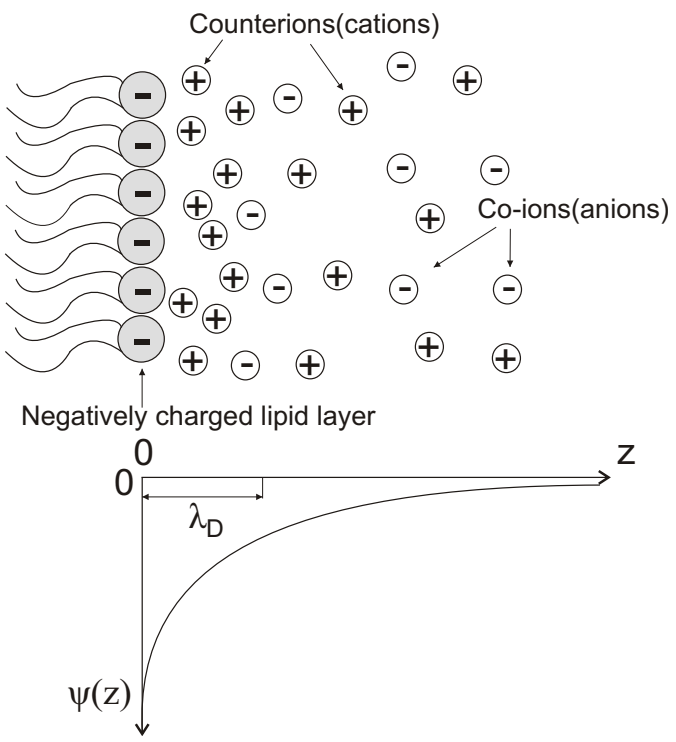

FIGURE 2.2.3. Schematic representation of a lipid layer, consisting of negatively charged lipids. A charged surface immersed in an aqueous solution is attracting (positive) counterions and creates a depletion zone of the (negative) coions. The graph shows the electrostatic potential $\phi(z)$ and Debye length $\lambda_{D}$. The $z$ axis denotes the distance from the surface.

each point $\vec{r}$ is the sum of the two ionic densities: $\rho(\vec{r})=e z_{+} n_{+}(\vec{r})+e z_{-} n_{-}(\vec{r})$, where $z_{+}\left(z_{-}\right)$is the valency of the counterions (co-ions).

The relation between the electric potential $\psi(\vec{r})$ and the charge distribution $\rho(\vec{r})$ at any point $\vec{r}$ is given by the Poisson equation

$$
\nabla^{2} \psi(\vec{r})=-\frac{\rho(\vec{r})}{\varepsilon_{0} \varepsilon_{w}}=-\frac{e}{\varepsilon_{0} \varepsilon_{w}}\left(z_{-} n_{-}+z_{+} n_{+}\right)
$$

The Poisson equation determines the electric potential for a given spatial charge distribution $\rho(\vec{r})$. Each ion density in the solution (in thermal equilibrium) obeys a Boltzmann distribution according to the electric potential it feels:

$$
n_{i}=n_{0}^{(i)} e^{-e z_{i} \psi / k_{B} T}
$$

where $i$ denotes different ionic species with valencies $z_{i}$. Combining Eqs. 2.2.2 and 2.2.3, we get the Poisson-Boltzmann equation ${ }^{2}$ which determines the potential $\psi$ self-consistently

$$
\nabla^{2} \psi(\vec{r})=-\frac{e}{\varepsilon_{0} \varepsilon_{w}}\left(z_{-} n_{0}^{(-)} e^{-e z_{-} \psi(\vec{r}) / k_{B} T}+z_{+} n_{0}^{(+)} e^{+e z_{+} \psi(\vec{r}) / k_{B} T}\right)
$$

\footnotetext{
${ }^{2}$ The assumptions which led to the derivation of the Poisson-Boltzmann equation 2.2 .4 can be summarized as follows: the ionic charge distributions are smeared out and are represented as smoothly varying functions. The discrete nature of the ions is not taken into account and no other molecular interaction between the ions and solvent molecules (water) is considered. Moreover, the Poisson-Boltzmann theory does not take into account any charge-charge correlations. Physical observables like the charge distributions are replaced by their thermal averages and, in this sense, resemble mean-field results [35].
} 
Generally speaking, the Poisson-Boltzmann theory is a good approximation in most physiological conditions, especially for monovalent ions and for surface potentials which are not too large.

In the situation where the system is in contact with a 1:1 electrolyte reservoir (e.g., $\mathrm{Na}^{+} \mathrm{Cl}^{-}$), $n_{0}^{( \pm)}=n_{0}$ is the electrolyte concentration in the reservoir (the bulk electrolyte concentration). The Poisson-Boltzmann equation 2.2.4 is then reduced to:

$$
\nabla^{2} \psi(\vec{r})=\frac{2 e n_{0}}{\varepsilon_{0} \varepsilon_{w}} \sinh \left(e \psi(\vec{r}) / k_{B} T\right)
$$

For valencies in Eq. 2.2.4 $z_{ \pm}= \pm 1$ is inserted. Divalent ions such as $\mathrm{Ca}^{2+}$ have important consequences on the electrostatics of membranes [37]. Most of those consequences go beyond the continuum approach of the Poisson-Boltzmann theory [35].

An approximation to the full Poisson-Boltzmann equation 2.2.4) is its linearized version, justified for surface potentials which are smaller than $25 \mathrm{mM}$ at room temperature [38]

$$
\nabla^{2} \psi(\vec{r})=\lambda_{D}^{-2} \psi
$$

where $\lambda_{D}=\left(2 e^{2} n_{0} / \varepsilon_{0} \varepsilon_{w} k_{B} T\right)^{-1 / 2} \sim n_{0}^{-1 / 2}$ is called the Debye-Hückel screening length. It varies from about $3 \AA$ for a $1 \mathrm{M}$ of 1:1 electrolyte like $\mathrm{NaCl}$ to about $1 \mu \mathrm{m}$ for pure water (due to the presence of $\mathrm{H}^{+}$and $\mathrm{OH}^{-}$ions even in pure water with an ionic strength of about $\left.10^{-7} \mathrm{M}\right)[\mathbf{3 8}]$.

The electric field is related to the surface charge density $\sigma$ by the electrostatic boundary conditions at $\mathrm{z}=0$

$$
\left.\frac{\partial \psi}{\partial z}\right|_{z=0}=-\frac{\sigma}{\varepsilon_{0} \varepsilon_{w}}>0 .
$$

Eq. 2.2.4 can be solved with this boundary condition 2.2.7 and with the assumption $n_{ \pm}(\infty)=$ $n_{0}$. The resulting potential is

$$
\psi(z)=-\frac{2 k_{B} T}{e} \ln \frac{1+\gamma e^{-z / \lambda_{D}}}{1-\gamma e^{-z / \lambda_{D}}}
$$

where the parameter $\gamma$ is the positive root of the quadratic equation:

$$
\gamma^{2}+\frac{2 \mu_{G C}}{\lambda_{D}} \gamma-1=0
$$

where $\mu_{G C}=e /\left(2 \pi|\sigma| l_{B}\right)$ is the so-called Gouy-Chapman length, with the Bjerrum length $l_{B}=$ $e^{2} / 4 \pi \varepsilon_{0} \varepsilon_{w} k_{B} T$. The Gouy-Chapman length $\mu_{G C}$ measures the extent of the counter-ion layer next to a charged surface and the Bjerrum length $l_{B}$ measures the distance at which two unit charges interact with thermal energy $k_{B} T$.

The surface potential $\psi_{S}=\psi(0)$ is related to $\gamma$ by

$$
\psi_{S}=-\frac{4 k_{B} T}{e} \operatorname{arctanh}(\gamma) .
$$


In the limit of a strong electrolyte, the surface potential $\psi_{S}$ is small enough so that a linearization of the Poisson-Boltzmann equation can be justified [35]. By either solving directly the linear Poisson-Boltzmann equation 2.2.6, or substituting the small $\psi_{S}$ limit in Eqs. 2.2.8 2.2.10 one obtains

$$
\psi(z)=\psi_{S} e^{-z / \lambda_{D}}=-\frac{4 \gamma k_{B} T}{e} e^{-z / \lambda_{D}}
$$

One can see from Eq. 2.2.11 that the 'diffusive layer' of ions in the solution is characterized by a 'thickness' $\lambda_{D}$.

2.2.2. Elastic properties of fluid-like membranes. The continuum model for the elastic energy of fluid-like membrane was proposed by Helfrich in analogy to smectic phase of liquid crystals [39]. The phenomenological elastic energy is expressed as an integral over the membrane area

$$
F_{b}=\frac{1}{2} k \int\left(c_{1}+c_{2}-c_{0}\right)^{2} d S+k_{G} \int c_{1} c_{2} d S
$$

where $k$ and $k_{G}$ are the mean and Gaussian elastic moduli, respectively. The two principal curvatures are $c_{1}$ and $c_{2}$ whereas $c_{0}$ is the the spontaneous curvature. For a single amphiphilic layer $c_{0}$ expresses the internal tendency of the layer to curve towards the water, $c_{0}>0$, or away from the water, $c_{0}<0$. The tendency to curve is a result of the different molecular structure and interactions of the head and tail moieties of the amphiphiles. For bilayer membranes composed of two identical layers $c_{0}=0$ from symmetry reasons. But $c_{0}$ can be non-zero if the composition of the two layers of the membrane differs. The membrane also is assumed to be incompressible [35].

\subsection{Solid supported biomimetic lipid bilayers}

Supported lipid bilayers (SLBs) have been developed as an alternative membrane model system to complement studies that can be performed on lipid monolayers or lipid vesicles [13]. Solid supports open the possibility for using surface specific analytical techniques not available for black lipid membranes [40].

Supported bilayers may be prepared by three different techniques: (1) In the LangmuirBlodgett-Schäfer method [13], a monolayer is first transferred from the air-water interface of a Langmuir trough to the hydrophilic support at a constant surface pressure of $32-36 \mathrm{mN} / \mathrm{m}$, that is, the bilayer equivalence pressure. A second monolayer is deposited by horizontal apposition of the substrate to a monolayer at the same surface pressure. (2) The second method is to fuse vesicles on a hydrophilic substrate [41]. A dispersion of small unilamellar vesicles spontaneously spreads and forms a continuous planar bilayer when brought into contact with a hydrophilic substrate. (3) The third method is a combination of the first two, where the vesicles are spread on a preexisting supported monolayer [42].

Vesicle fusion is one of the most convenient ways of creating supported lipid bilayers. The mechanism by which supported bilayers are formed from vesicles has been studied theoretically by 
Lipowsky and Seifert $[43,44]]^{3}$. The model, these authors suggested, is that vesicles deform upon adsorption and rupture if the free energy of the deformed bound vesicle are higher than the free energy of a single-bilayer disk [45]. Adsorption of a vesicle to an attractive wall is governed by the interplay between the (favorable) adhesion energy and the (unfavorable) bending energy. The energy gained by the vesicle upon adhering to the wall can be expressed as $F_{a}=-W A^{*}=-W 4 \pi R^{2}$ ( $A^{*}$ is the contact area, $W$ is the effective contact potential and $R$ is the radius of the vesicle). The deviation of the vesicle from a spherical shape (bending) upon adhesion, expressed as $F_{b}=$ $\frac{1}{2} k \int d A\left(c_{1}+c_{2}\right)^{2}$ (where $k$ is the bending rigidity of the bilayer, $c_{1}$ and $c_{2}$ are the two principle curvatures and the integration is performed over the surface area $A$ of the vesicle), depends on the bending modulus of the membrane $k$ but not on the vesicle size. Therefore, for a given $W$, the gain due to the size-dependent adhesion energy becomes higher than the cost due to the sizeindependent bending energy when the size of the vesicle $R$ is larger than the critical radius $R_{c}$ :

$$
R_{c}=(2 k / W)^{1 / 2}
$$

For strong adhesion, or, equivalently, for large vesicles, a simplification occurs because the scale-invariant curvature energy becomes irrelevant compared with the gain in contact energy. With respect to the formation of disks from adsorbed vesicles, the energy of the initial state (a bound vesicle, which in the limit of large $R$ and/or $W$ becomes flattened and resembles a pancake) has to be compared with that of the final state (a bound disk). According to Seifert:

$$
\begin{gathered}
F_{b v}=-2 \pi W R^{2}+2 \pi g(2 k W)^{1 / 2} R \\
F_{b d}=-4 \pi W R^{2}+4 \pi \Sigma R
\end{gathered}
$$

where $F_{b v}$ and $F_{b d}$ are the free energies of the bound vesicle and the disk, respectively. $R$ is the radius of the vesicle (disk radius $R_{d}=2 R$ ), $\Sigma$ is the line tension along the circumference of the disk and $g \simeq 2.8$ is a numerical constant [43, 44]. A bound vesicle will rupture if $F_{b d}<F_{b v}$, which leads to an expression for the rupture radius $R_{r}=\left[2 \Sigma-g(2 k W)^{1 / 2}\right] / W$. Isolated vesicles with $R \geq R_{r}$ will rupture while isolated vesicles with $R<R_{r}$ will remain intact. Small vesicles floating in the solution can fuse with those stuck on the substrate to form cylindrical shaped vesicles [46]. Eventually these bigger vesicles rupture to form planar membranes. Fig. 2.3.1 illustrates this process. There are also other mechanisms for this transition process, but the one shown in Fig. 2.3.1 is the main route. Provided the density of adsorbed vesicles is sufficiently high, such a process can propagate in a cascade of rupture events across several neighboring vesicles and leads to formation of extended bilayer patches. Furthermore, adjacent bilayer patches usually coalesce in order to minimize their edge length. These effects increase the size of individual bilayer patches and the overall bilayer coverage and will, in the ideal case, lead to a complete SLB [47].

\footnotetext{
${ }_{3}^{3}$ Seifert and Lipowsky [43, 44] elaborated a model in which the lipid bilayer is regarded as a macroscopic sheet with a certain curvature-dependent free energy [39]. In their model the structure of the lipid bilayer does not play a role and the Gaussian bending modulus $k_{G}=0$. They introduced an effective contact potential that, together with the interaction area between the vesicle and the substrate, determines the total adhesion energy. In addition some constraints for the volume and area of the vesicle were applied.
} 


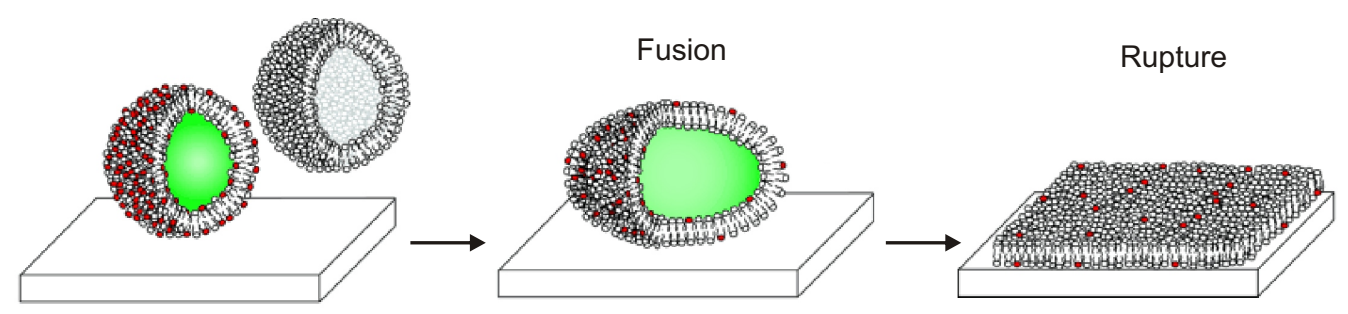

FIGURE 2.3.1. Proposed bilayer formation schematics [46]. Adsorbed vesicles fuse among themselves until a critical size is reached, then rupture to form bilayer disks.

Kasemo and co-workers [48] have reported an interesting effect of the cooperative action of surface-bound vesicles. They showed that isolated vesicles of egg-PC remain intact when bound to a silica support and that a certain surface density of vesicles (critical vesicular coverage) is required to initiate the decomposition of surface-bound vesicles into bilayer patches.

Interaction between membranes and surfaces involves electrostatic and hydration forces as well as attractive contributions from long-range van der Waals forces. An energetic minimum tightly traps the membrane near the surface [13]. There are extended theoretical and experimental efforts to understand and control the deposition and properties of the SLBs $[45,47,48,449,50,51$, 52, 53]. Despite these efforts, the precise balance of forces governing the formation and interaction between the membrane and surface is not fully understood [52].

The factors affecting formation and stability of SLBs include: the nature of the support (its surface charge, chemical composition and roughness), the lipid vesicles (their composition, charge, size, and physical state), as well as the aqueous environment (its composition, $\mathrm{pH}$ and ionic strength) $[\mathbf{5 2}, \mathbf{5 0}]$.

Solid support. In order to prepare high quality membrane (i.e. little defects and high lipid mobility) the surface should be hydrophilic, smooth and clean. A number of studies report difficulties to form SLBs on surfaces as gold, $\mathrm{TiO}_{2}$ or platinum [52]. The most common substrates are mica and silicon based materials, such as glass, $\mathrm{SiO}_{2}$ or $\mathrm{Si}_{3} \mathrm{~N}_{4}$. It was shown that the roughness on the nanometer range only slightly affect the SLBs formation [47, 49]. The surface manufacturing and preparation may influence the kinetics of lipid deposition. The charge of the support (for example the hydroxilation state of silica surface) is influenced by the manufacturing procedure, exposure to high temperature or to basic solutions.

Electrostatic interactions. Several studies have pointed out the influence of the charge of support and lipids as well as the ionic strength on the absorption of vesicles [50, 54, 55]. Adjustment in the $\mathrm{pH}$ or in the ionic strength are expected to optimize the formation of SLBs for given surface and a given lipid composition [56, 55]. Silicon oxide surface exhibits negative charge at $\mathrm{pH}$ values $>3.5$ and almost no charge (fully protonated species) at lower $\mathrm{pH}$ values [57]. The deposition and spreading of the negatively charged vesicles on the negatively charged support may be favored at high ionic strengths [56]. 
Calcium ions. The deposition of vesicles composed of charged and zwitterionic lipid species was found to be enhanced in the presence of calcium ions [47]. It is likely due to the ability of $\mathrm{Ca}^{2+}$ ions to bridge negatively charged entities, such as carboxylate groups and phosphate groups in charged and zwitterionic lipid species, or negative charges on the silica surface. Calcium, which is known to be a fusogenic agent, has a profound effect on the adsorption and rupture of vesicles $[\mathbf{5 0}, 45,[58]$. Calcium ions do not only participate in the screening of the charges, thereby modifying the electrostatic interactions, but they also directly interact with surfaces and lipids [58].

The fluidity of the membrane in terms of lateral self-diffusion is considered to be an important condition of biomimetic lipid membranes. The lateral diffusion coefficients of supported lipid bilayers vary in the range $1-5 \times 10^{-8} \mathrm{~cm}^{2} / \mathrm{s}$, depending on the substrate properties [59]. The water layer thickness between solid support and the bilayer was found by X-ray reflectivity method to be $3.5-6 \AA[\mathbf{6 0}, \mathbf{6 1}]$. Comparative studies of lipid diffusion in free giant unilamellar vesicles and mica supported lipid bilayers have shown that the diffusion coefficients of DOPC bilayers are reduced by a factor of two at the solid interface [59]. Rossetti et al. [62] have estimated the diffusion coefficients of 1- oleoyl-2-[6-[(7-nitro-2-1,3-benzoxadiazol4-yl)amino]dodecanoyl-sn-glycero-3-phosphocholine (NBD - PC) and 1-oleoyl-2-[6-[(7-nitro-21,3-benzoxadiazol-4-yl)amino]dodecanoyl-sn-glycero-3-phospho-L-serine (NDB-PS) in SPBs containing $20 \%$ DOPS (formed at room temperature on $\mathrm{SiO}_{2}$ in the presence of $\mathrm{Ca}^{2+}$ ) to be $\sim 1.1 \mathrm{x}$ $10^{-8} \mathrm{~cm}^{2} / \mathrm{s}$ for NDB-PC and $\sim 2.0 \times 10^{-8} \mathrm{~cm}^{2} / \mathrm{s}$ for NDB-PS.

One of the important questions is the interleaflet distribution of different lipid species in SLBs that are formed from vesicles containing lipid mixtures. Richter et al. [50] have found the distribution of DOPS in DOPC/DOPS (4:1) membrane supported on silica to be symmetrical, within the experimental error. In contrast, they have found asymmetrical distribution of DOPS on mica.

Furthermore, the desire to properly mimic the complex nature of two-dimensionally fluid plasma membranes is the driving force for the development of the polymer-cushioned, tethered, or pore-spanning lipid bilayers $[\mathbf{5 2}, 6 \mathbf{6 3}, 64]$.

\subsection{Colloidal nanoparticles}

Gold nanoparticles are now in widespread use for localizing cell components with immunochemistry and microscopy techniques. Suspensions of finely divided gold particles, often termed gold colloids, can be produced by chemical reduction of a gold salt in aqueous solution producing a supersaturated solution of elemental gold from which crystals of metallic gold condense out [65]. Careful manipulation of the reduction conditions enables the final size of the particles to be precisely controlled, yielding particles with diameters between 2 and $150 \mathrm{~nm}$ or more. Redgold colloids show a peak of absorption between 515 and $540 \mathrm{~nm}$ with the peak moving to larger wavelengths as the particle diameter increases.

In the absence of electrolytes the gold particles in suspension are quite stable because their negative surface charge ensures that they do not stick to each other. The surface charges are due to dissociation of salts such as $\mathrm{HAuCl}_{4}, \mathrm{HAuCl}_{2}, \mathrm{HAuOHCl}$ or $\mathrm{HAuOH}_{2}$, or alternatively, reducing 
agents adsorbed from reducing solution [65]. The addition of electrolytes to a gold colloid induces the particles to form aggregates, the color changing from red to blue or black within seconds.

Electrostatic interaction plays an important role in colloids. The linearized screening theory of Debye-Hücke always leads to a repulsive interaction between like-charged colloids in an aqueous solution. In the Derjaguin-Landau-Verwey-Overbeek (DLVO) theory [66, 67], the electrostatic part of the effective interaction between particles $U_{D L V O}(r)$ is given by

$$
U_{D L V O}(r)=\frac{Z^{2} e^{2}}{4 \pi \varepsilon}\left(\frac{e^{\kappa R}}{1+\kappa R}\right)^{2} \frac{e^{-\kappa D}}{D}
$$

where $Z, e, \kappa, R, \varepsilon$, and $D$ denote the surface charge of colloidal particles, the elementary charge of electron, the inverse of the Debye-Hückel screening length, the radius of colloidal particles, the dielectric constant of the medium, and the center-to center distance between two colloidal particles, respectively. The inverse screening length $\kappa$ is given by

$$
\kappa^{2}=4 \pi \lambda_{B} \sum_{j} n_{j} q_{j}^{2}
$$

where $\lambda_{B}=e^{2} / 4 \pi \varepsilon k_{B} T$ is the Bjerrum length and $n_{j}$ is the $q_{j}$-valent ion density. At high salt concentrations, when the electrostatics is sufficiently screened, the attractive dispersion forces result in flocculation.

2.4.1. Colloid-membrane interaction. Adhesion of colloidal particles to lipid membrane is usually caused by specific binding of ligands to membrane receptors or by electrostatic interactions. Lipid membranes are soft and self-assembled materials, able to simultaneously adjust their local charge density through compositional changes, as well as their curvature through elastic deformations. Electrostatic adsorption of colloids on a two-component membrane with ideal mixing properties can lead to compositional inhomogeneities at colloids adsorption sites [17]. Charges in such lipids differ in magnitude and location within the interface and can locally demix in response to charged colloids. If binding of charged colloids to a multi-component membrane results in a re-distribution of charged and uncharged lipids, this altered membrane state will in return affect the bilayer-nanoparticle interaction, so that strong non-linear effects can be expected. This effect is more pronounced in lipid mixtures with a tendency towards demixing than for ideal mixtures [68]. Ramos et al. [15] observed, that colloidal particles can form two-dimensional ordered colloidal crystals when they interact with surfactants of the opposite charge. Coulomb interactions lead to self-limited adsorption of the particles on the surface of vesicles formed by the surfactants. Adsorbed particles form ordered but fluid rafts on vesicle surfaces, and these form robust two-dimensional crystals. Schematic illustration of colloids enhanced microdomain formation in mixed lipid membranes is shown in Fig. 2.4.1.

Colloids are able to induce elastic deformations in charged membranes, giving rise to various phenomena such as membrane-mediated elastic interactions between colloids or wrapping of the membrane around the colloid [17, 69]. Koltover et al. [70] reported a light microscopy study of 


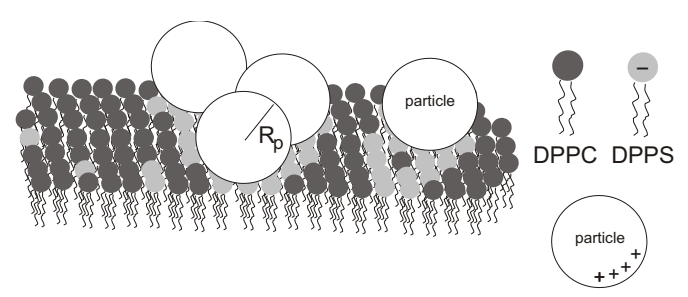

FIGURE 2.4.1. Schematic illustration of charged, two-component lipid membrane, consisting of neutral and negatively charged lipids. Electrostatic binding of oppositely charged colloidal particles can induce formation of colloid-coupled micro-domains.

interaction between colloidal particles bound onto flexible giant vesicle fluid membranes. Particles induced pinched shape deformations of the membrane and were driven to negative curvature regions on nonspherical vesicles. Membrane distortions were found to induce interparticle attraction with a range approximately equal to the particle diameter. Particles decorating membranes aggregate into finite-sized two-dimensional close packed aggregates. Deserno and Bickel [69] theoretically studied elastic deformations of a fluid membrane induced by an adhering spherical colloidal particle within the framework of a Helfrich energy. They presented structural wrapping phase diagram and predicted transition lines between partially and fully wrapping of particles. One particular consequence of the wrapping scenario is that colloid engulfment is extremely sensitive to the size of the particle. As long as the tension is low enough, a large sphere is wrapped much easier than a small one. Schematic illustration of colloid induced lipid membrane deformation is shown in Fig. 2.4.2.

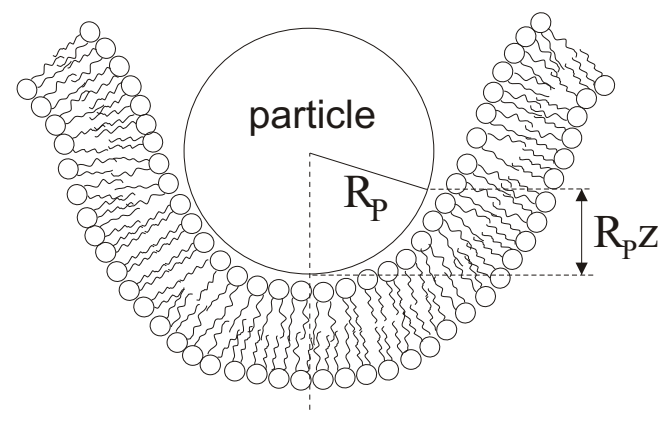

FIGURE 2.4.2. Schematic illustration of a bending deformation in a charged membrane induced by electrostatic binding of oppositely charged colloid of radius $R_{P} . z$ denotes the degree of wrapping.

Noguchi and Takasu [71] used a Brownian dynamics simulation to study the interaction of bilayer vesicles and adhesive nanoparticles. They have shown that nanoparticles induce structural changes of vesicles: budding, stalk formation, and fission.

Cytotoxicity of gold nanoparticles has been observed at high concentrations. Studies of Goodman et al. [72] have shown that cationic particles are moderately toxic, whereas anionic particles are quite nontoxic. The toxicity of cationic gold nanoparticles may be related to their strong electrostatic attraction to the negatively charged bilayer. 


\section{CHAPTER 3}

\section{Materials and Preparation Techniques}

\subsection{Small unillamelar vesicles}

Chemicals: 1,2-Dipalmitoyl-sn-Glycero-3-Phosphatidylserine (DPPS), 1,2-Dioleoyl-3-Trimethylammonium-Propane (DOTAP), 1,2-Dioleoyl-sn-Glycero-3-Phosphatidylcholine (DOPC), 1,2-Dioleoyl-sn-Glycero-3-Phosphatidylserine (DOPS), 1,2-Dipalmitoyl-sn-Glycero-3-Phosphatidylcholine (DPPC), 1,2-Dimyristoyl-3-Phosphatidylcholin (DMPC) were obtained from Avanti Polar Lipids (Alabaster, AL, USA). Texas red-DHPE (N-(Texas Red sulfonyl)-1,2-dihexadecanoyl-sn-glycero-3-phosphoethanolamine, triethylammonium salt) was obtained from Molecular Probes (Eugene, OR). Hepes (H-4034), NaCl (S-9888), sodium dodecyl sulfate (05030), chloroform (25693) were purchased from Sigma-Aldrich (Germany), and $\mathrm{CaCl}_{2}$ from Merck $\mathrm{KGaA}$ (Germany). Chemical structures, molecular weights and transition temperatures of the phospholipids used in this study are shown in Fig. 3.1.1.

Buffer solutions: A buffer solution (A), contained of $150 \mathrm{mM} \mathrm{NaCl}$ and $10 \mathrm{mM} \mathrm{HEPES} / \mathrm{NaOH}$, $\mathrm{pH} 7.4$ was prepared with ultrapure water and a buffer (B) was prepared by adding $2 \mathrm{mM} \mathrm{CaCl}$ to the buffer (A).

Lipids were mixed in desired amounts (DPPC/DPPS (molar ratio 4:1, 1:1), DOPC/DOPS (4:1), DOPC/DOTAP (9:1)) and dissolved in chloroform. The solvent was evaporated, followed by drying in a vacuum oven for $24 \mathrm{~h}$ in order to remove all traces of the solvent. DPPC/DPPS and DOPC/DOPS lipid mixtures were resuspended in a buffer solution (B) at total concentration $0.75 \mathrm{mg} / \mathrm{ml}$. DOPC/DOTAP lipid mixture was resuspended in $10 \mathrm{mM}$ HEPES buffer at the total concentration $2.5 \mathrm{mg} / \mathrm{ml}$ and DOPC in buffer (A) at the concentration $0.75 \mathrm{mg} / \mathrm{ml}$. Suspensions were vortexed for $1 \mathrm{~min}$. Small unilamellar vesicles (SUVs) were obtained by sonicating lipid solutions with a tip sonicator (Sonoplus; Bandelin, Berlin, Germany) for 15-30 min, followed by centrifugation in an Eppendorf centrifuge $(10 \mathrm{~min}$ at $14.000 \mathrm{x} \mathrm{g})$ to remove titanium particles. Before use, vesicle suspensions, except DOPC/DOTAP, were diluted to final concentrations 0.1 $\mathrm{mg} / \mathrm{ml}$ in the respective buffers.

Dynamic light scattering: The size of the vesicles was measured by dynamic light scattering at a $90^{\circ}$ scattering angle with ALV-CGS3, ALV Correlator 5000 (Langen, Germany). Sample was filled in shortened, round NMR-tube with outer diameter of $4.97 \mathrm{~mm}$ and inner diameter of 4.20 $\mathrm{mm}$. The final concentration of the used vesicle solutions was $1 \mathrm{mg} / \mathrm{ml}$. The diameter of DOPC vesicles was found to be $31.4 \pm 3.15 \mathrm{~nm}$ and the diameter of DOPC/DOPS (4:1) vesicles was found to be $29.5 \pm 2.95 \mathrm{~nm}$. 
(a) $\mathrm{DOPC}, \mathrm{MW}=786.150, \mathrm{~T}_{\mathrm{m}}=-20^{\circ} \mathrm{C}$

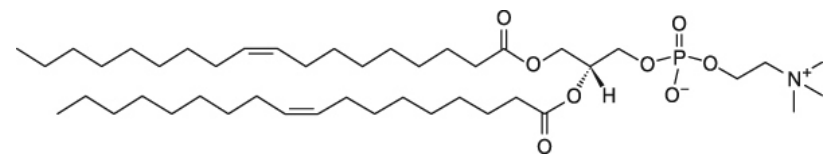

(b) DOPS, MW $=810.040, \mathrm{~T}_{\mathrm{m}}=-11^{\circ} \mathrm{C}$

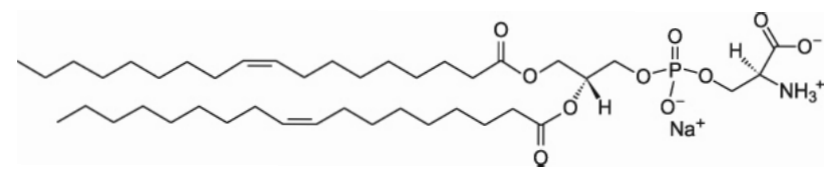

(c) $\mathrm{DPPC}, \mathrm{MW}=734.050 \mathrm{~T} \mathrm{~m}=41.5^{\circ} \mathrm{C}$

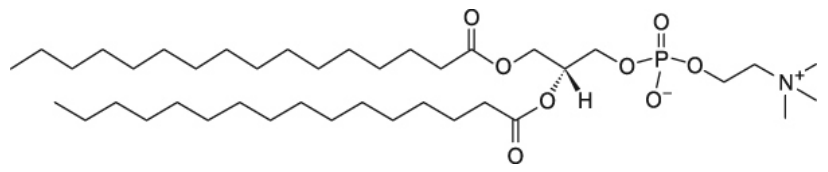

(d) DPPS, $\mathrm{MW}=757.960, \mathrm{~T}_{\mathrm{m}}=54^{\circ} \mathrm{C}$

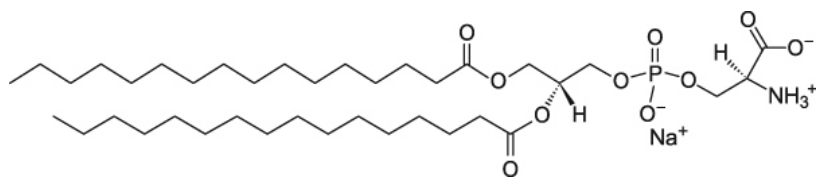

(e) DOTAP, $\mathrm{MW}=698.550, \mathrm{~T}_{\mathrm{m}}=-8^{\circ} \mathrm{C}$

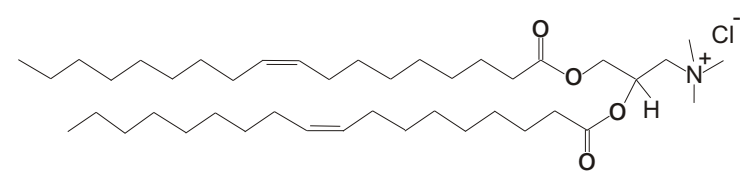

(f) DMPC, MW $=677.940, \mathrm{~T}_{\mathrm{m}}=23^{\circ} \mathrm{C}$<smiles>CCCCCCCCCCCCCCCCCCC(=O)OCC(COP(=O)([O-])OCC[N+](C)(C)C)OC(=O)CCCCCCCC</smiles>

(g) Texas Red DHPE (582/601), MW = 1381.850

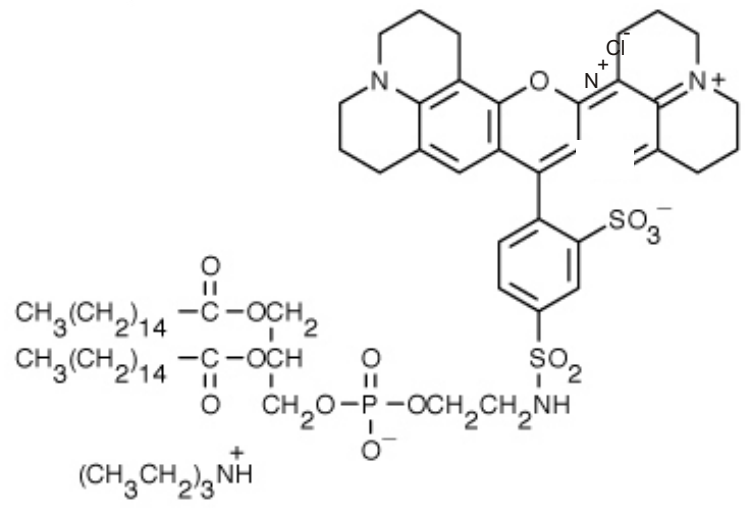

FigURE 3.1.1. Chemical structures, molecular weights and transition temperatures $([\mathbf{2 0}, \mathbf{7 3}])$ of the phospholipids used in this study. Structural sketches are adapted from Avanti Polar Lipids (www.avantilipids.com). 


\subsection{Two-component solid supported lipid membranes}

Substrates: (100)-silicon wafers (Silchem; Freiberg, Germany) were cut to the size $15 \times$ $10 \mathrm{~mm}^{2}$. The silicon nitride $\left(\mathrm{Si}_{3} \mathrm{~N}_{4}\right)$ windows were obtained from Silson Ltd (Northampton, England). The size of silicon nitride foils was $10 \times 10 \mathrm{~mm}^{2}$, with the membrane size $0.5 \times 0.5$ $\mathrm{mm}^{2}$, the membrane thickness $100 \mathrm{~nm}$ and the frame thickness $200 \mu \mathrm{m}$. The size of silicon foils was $15 \times 15 \mathrm{~mm}^{2}$ and the membrane thickness $200 \mathrm{~nm}$. Photograps of the silicon and the silicon nitride foils are shown in Fig. 3.2.1.

a)

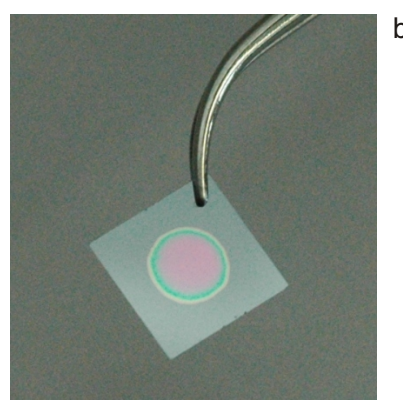

b)

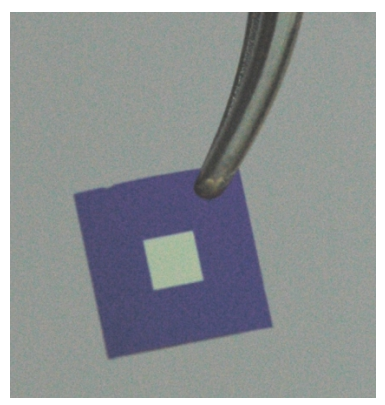

FIGURE 3.2.1. Photographs of the silicon (a) and the silicon nitride (b) foils.

Substrate preparation: Silicon wafers were cleaned by two 20 min cycles of the ultrasonic bath in $2 \%$ sodium dodecyl sulfate solution or by three $15 \mathrm{~min}$ cycles of the ultrasonic bath in methanol, followed by two $20 \mathrm{~min}$ cycles in ultrapure water (18 $\mathrm{M} \Omega \mathrm{cm}$, Millipore, Bedford, MA), and drying under nitrogen flow. The $\mathrm{Si}_{3} \mathrm{~N}_{4}$ and silicon foils were carefully cleaned subsequently in methanol, aceton and isopropanol, followed by extensive washing with ultrapure water, and drying under stream of nitrogen. Finally, the surface of wafers was rendered hydrophilic and cleaned of organic contaminants by etching in plasma cleaner (Harrick Plasma, NY, USA) for 3 min.

Lipid bilayers were prepared on cleaned substrates by vesicle fusion method [41, 49]. Methods discussed in the following were used to examine the surface of the substrates, to image supported lipid bilayers and to study the structure of two-component lipid membranes.

Atomic force microscopy (AFM): AFM was performed on a NanoWizard AFM (JPK Instruments, Berlin, Germany) operated in tapping mode in water. Ultrasharp silicon tips (NT-MDT, Moscow, Russia) were used. The bilayer was allowed to self-assemble by placing $1 \mathrm{ml}$ drop of vesicle suspension over the cleaned silicon wafer in a teflon dish (Fig. 3.2.2) for 30 - 60 minutes, followed by rinsing away the excess vesicles.

Fluorescence microscopy: Membranes were fluorescently labeled by adding $0.1 \mathrm{~mol} \%$ Texas red DHPE $\left(\lambda_{e x} / \lambda_{e m}=582 / 601 \mathrm{~nm}\right)$. The bilayer was allowed to self-assemble by placing $1 \mathrm{ml}$ drop of vesicle suspension over the cleaned silicon foil in a Petri dish for 30-60 minutes, followed by rinsing away the excess vesicles. The sample was kept in the buffer all the time during the preparation and measurement. Fluorescence microscopy was performed on 1) an AxioTech Vario (Zeiss, Jena, Germany), which was equipped with a 40x water immersion objective (ACHROPLAN, NA $=0.8$ water, Zeiss, Hamburg, Germany) and a filter set allowing the excitation of fluorescence at around $560 \mathrm{~nm}$ and emission at around $630 \mathrm{~nm}$ (filter no. 45, Zeiss, Jena, Germany), and 2) an 


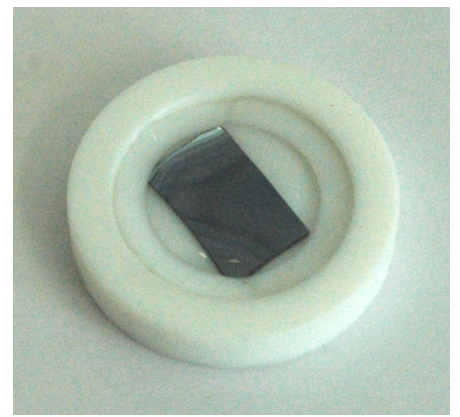

FIGURE 3.2.2. Teflon chamber used for atomic force microscopy experiments.

Axio Observer.Z1 (Zeiss, Jena, Germany), equipped with a 40× objective (Plan-Neofluar, NA = $0.6)$ and a filter set 560/630 $\mathrm{nm}$ (Colibri).

X-ray reflectivity: Cleaned silicon wafers with dimensions $15 \pm 10 \mathrm{~mm}$ were placed into a chamber with kapton windows (see Fig. 5.2.5 in chapter 5), designed for this reflectivity measurement. The chamber frame was made of teflon that was chemically inert and easy to clean. The chamber was filled with lipid vesicle solution heated above the transition temperature of the lipids used and incubated for $30 \mathrm{~min}$. In the case of DOPC/DOTAP lipid mixture the incubation time was $10 \mathrm{~h}$ to ensure a symmetric partitioning into both leaflets of the bilayer, which might be kinetically more difficult to achieve for cationic lipids [74]. Excess vesicles were rinsed away, while the membrane remained hydrated all the time during preparation and measurement. The temperature $23.8{ }^{\circ} \mathrm{C}$ of the chamber was kept by a flow of $1: 2$ glycol:water mixture from a temperature-controlled reservoir (Julabo, Seelbach, Germany).

(a)

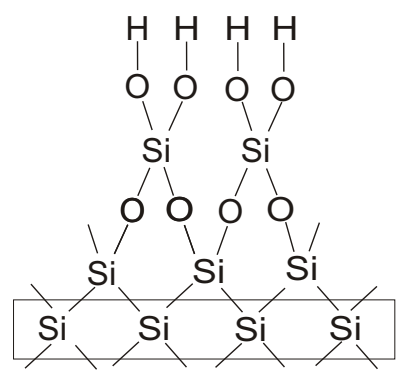

(b)

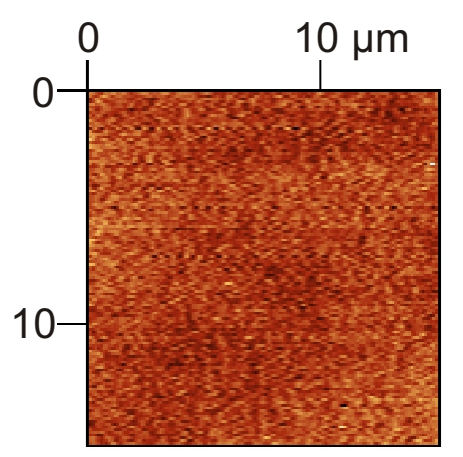

FIGURE 3.2.3. (a) The structure of a vertical slice through the $\mathrm{Si} /$ native $\mathrm{SiO}_{2}$. The terminating chemical groups of hydrophilic silica are the Si-OH silanol groups, where the hydroxyl group -OH is the terminating unit. (b) The atomic force microscopy image ( $15 \times 15 \mu \mathrm{m}^{2}$ ) of a bare silicon wafer. A surface rms roughness was estimated to be of the order of $2-3 \AA$.

The bare silicon wafer has a surface covered with a thin native oxide layer. The structure of $\mathrm{Si} /$ native $\mathrm{SiO}_{2}$ is shown in Fig. 3.2.3 (a). The bulk silicon is in crystalline form, while the terminating native oxide layer is composed of amorphous silica. Usually, this layer has a thickness of 6-20 [75]. Terminating chemical groups of hydrophilic silica are Si-OH silanol groups, 
where the hydroxyl group -OH is the terminating unit. Surface roughness of the substrate was examined by tapping mode AFM. Typical AFM image of a bare silicon wafer is shown in Fig. 3.2.3 (b). A surface roughness was calculated from AFM images of different parts of the sample as a root-mean-square ( $\mathrm{rms}$ ) value of the height irregularities computed from data variance, using the image analysis software Gwyddion 2 The surface rms roughness was estimated to be of the order of $2-3 \AA$.

Fluorescence image of the spreading of a membrane front (DOPC) across a silicon oxide substrate is shown in Fig. 3.2 .4 .

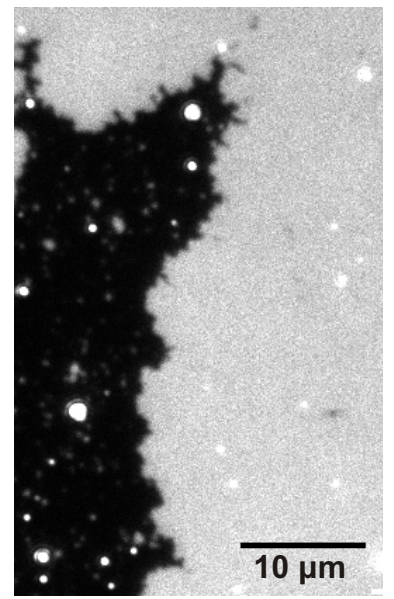

FIGURE 3.2.4. Fluorescence gray-scale image of the spreading of a membrane front (DOPC) across a silicon foil after vesicle rupture. Brighter fluorescence intensities correspond to the preformed bilayer or to floating lipid vesicles and darker to the Si foil.

Gray-scale fluorescence images of the various lipid mixtures with low positive (DOPC/DOTAP (1:9)), neutral (DOPC) and negative charge (DOPC/DOPS (4:1), DPPC/DPPS (4:1), (1:1)) are shown in Fig. 3.2.5. The lipid bilayers were deposited by vesicle fusion on Si foils. Fluorescence microscopy was carried out at a constant temperature $23.8^{\circ} \mathrm{C}$. The main transition temperatures of DOPC, DOTAP and DOPS are below $0{ }^{\circ} \mathrm{C}[\mathbf{2 0}]$, therefore all compositions of these lipids, we used in our study, are expected to be in the fully hydrated fluid phase at $23.8{ }^{\circ} \mathrm{C}$. Fig. 3.2.5 (a, b, c) shows images of substrates highly covered with DOPC, DOPC/DOPS (4:1) and DOPC/DOTAP (9:1) lipid bilayers, respectively. The phase transitions of DPPC and DPPS from the ordered gel phase to the disordered liquid-crystalline phase are reported in the literature to occur at $41.4{ }^{\circ} \mathrm{C}$ and $54{ }^{\circ} \mathrm{C}[20]$. The deposition of DPPC/DPPS (4:1), (1:1) bilayers onto substrates was done at $60{ }^{\circ} \mathrm{C}$, followed by slow cooling of samples to $23.8^{\circ} \mathrm{C}$ (gel phase). Fluorescence images of DPPC/DPPS (4:1) and DPPC/DPPS (1:1) bilayers are shown in Fig. 3.2.5 (d, e), respectively. In these images dark areas represent bare silicon surfaces, and the light areas represent lipid bilayers. Defects in gel phase bilayers were observed. This is believed to be inevitable due $1_{r m s}=\sqrt{\frac{1}{N-1} \sum_{i}\left(x_{i}-x\right)^{2}}$, where $x$ is the average height value within the given area, $x_{i}$ is the current height value and $N$ is the number of points within a given area.

${ }^{2}$ http://gwyddion.net/ 
to the considerable shrinkage of the area per lipid molecule in the gel phase: 15 - $20 \%$ less than in the fluid phase [76, 77].
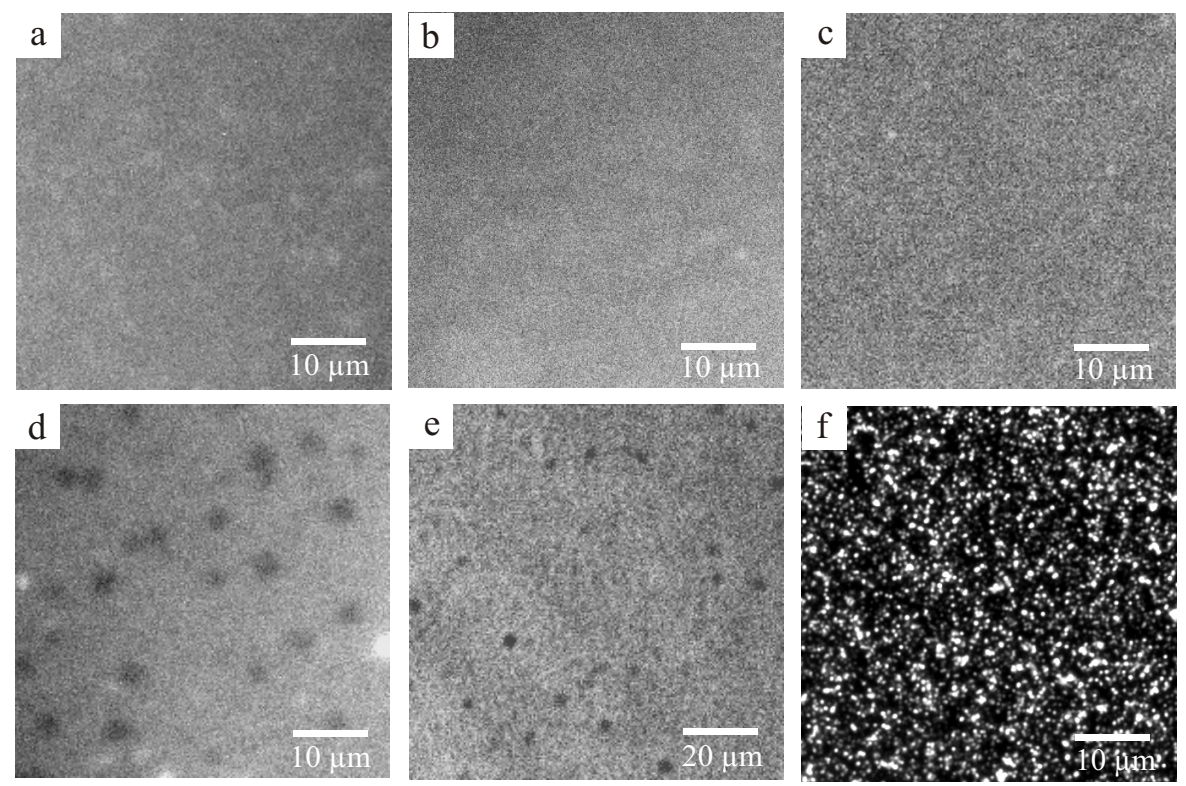

FIGURE 3.2.5. Gray-scale fluorescence images of the different lipid mixtures at room temperature covering silicon substrates: (a) DOPC, (b) DOPC/DOPS (4:1), (c) DOPC/DOTAP (9:1), (c) DPPC/DPPS (4:1), (d) DPPC/DPPS (4:1), (e) DPPC/DPPS (1:1) and (f) DPPC/DPPS (4:1) in the buffer without $\mathrm{Ca}^{2+}$ ions. (Abbreviations are listed in the List of Abbreviations.)

Fig. 3.2.5 (f) shows the fluorescence image of incomplete vesicle fusion of DPPC/DPPS (4:1) vesicles in the buffer solution (A) without $\mathrm{Ca}^{2+}$ ions. The deposition of vesicles composed of charged and zwitterionic lipid species was found to be enhanced in the presence of calcium ions [47]. This is most likely due to the ability of $\mathrm{Ca}^{2+}$ ions to bridge negatively charged entities such as the carboxylate groups and the phosphate groups in charged and zwitterionic lipid species, or negative charges on the silica surface. The most important effect of $\mathrm{Ca}^{2+}$ was suggested to be due to the well-known fact that this ion is a strong fusogenic agent, promoting the fusion of cells and liposomes [78]. The AFM image of the incomplete DPPC/DPPS (4:1) layer is shown in Fig. 3.3.2.

\subsection{Suppported lipid membrane mixed with amidine latex colloids}

Colloids: The positively charged polystyrene (PS) latex particles (amidine charge groups) were purchased from Interfacial Dynamics Corp. (Portland, OR). Latex colloids are reported by the manufacturer to have a mean radius of $0.195 \mu \mathrm{m}$ and a density of $1.055 \mathrm{~g} / \mathrm{cm}^{3}$. A scanning electron microscopy (SEM) image of amidine latex particles is shown in Fig. 3.3.1. (a).

A sample of air-dried PS microspheres was prepared. The droplet of colloid solution was deposited on the $\mathrm{Si}_{3} \mathrm{~N}_{4}$ membrane and allowed to dry out on the surface. The sample was examined by using scanning transmission X-ray spectro-microscopy. 
a)

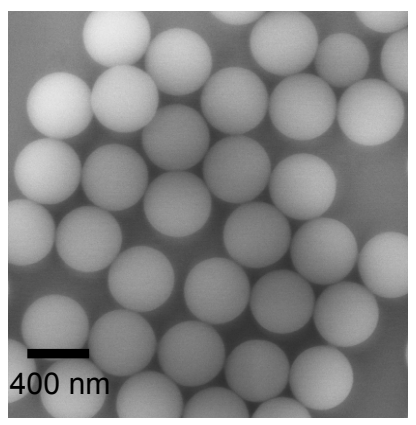

b)

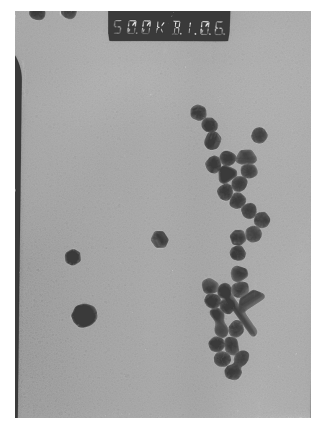

c)

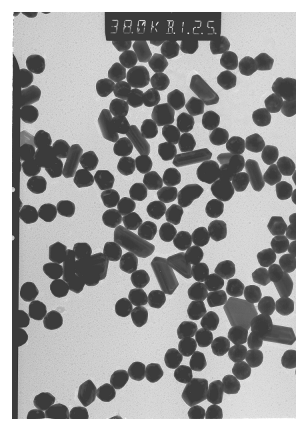

FIGURE 3.3.1. Scanning electron microscopy images showing (a) charged amidine polystyrene particles of diameter $\sim 390 \mathrm{~nm}$, (b) poly(sodium-4-styrene sulfonate)/poly(allylamine) hydrochloride coated gold particles of diameter $\mathrm{d}=64$ $\pm 5 \mathrm{~nm}$ and $(\mathrm{c}) \mathrm{d}=103 \pm 7 \mathrm{~nm}$.

DPPC/DPPS (4:1) lipid vesicles were prepared in the buffer solution (A) without $\mathrm{Ca}^{2+}$ ions as described above. The drop of lipid vesicles was placed over a cleaned $\mathrm{Si}_{3} \mathrm{~N}_{4}$ window and incubated at $60{ }^{\circ} \mathrm{C}$ for $30 \mathrm{~min}$. Excess vesicles were rinsed away, while the lipid membrane remained hydrated all the time during preparation and measurement. The vesicle fusion then results in an incomplete coverage shown in Fig. 3.3.2 (a). Incomplete coverage was found to be an advantage for scanning transmission X-ray spectro-microscopy experiments because of lateral contrast variation. The lipid layer thickness was measured to be $\sim 10 \mathrm{~nm}$, what might indicate the intermediate step in a single bilayer formation process: two bilayers stacked on top of each other.

The process of supported lipid bilayer formation can be generally divided into a number of steps: adhesion, vesicle flattening, rupture, and finally formation of a planar single-bilayer structure $[\mathbf{7 9},[50]$. In the first step the bound vesicle is believed to collapse onto itself resulting in a double-bilayer structure. This process continues until the entire vesicle transforms into two bilayers stacked on the top of each other [79]. The transition to a single bilayer takes place in a way that may be interpreted as sliding, or rolling motion $[\mathbf{5 4}, \mathbf{5 0}]$.

The deposited DPPC/DPPS (4:1) lipid layer was then incubated with added positively charged PS latex microspheres at volume fractions of about $0.04 \%$. After 30 minutes, the unbound microspheres were gently rinsed off with the buffer solution. The prepared sample was then capped by a second $\mathrm{Si}_{3} \mathrm{~N}_{4}$ window to form a wet cell (Fig. 3.3.2(b)). The wet cell was sealed and glued with a nail polish onto a metal shim, which was then fixed between two specimen chamber rings. The specimen chamber shown in Fig. 6.4.2 in chapter 6 was used for scanning transmission X-ray microscopy experiments at BESSY II.

\subsection{Suppported lipid membrane mixed with gold colloids}

Colloids: The positively charged poly(sodium-4-styrene sulfonate) (PSS)/poly(allylamine) hydrochloride (PAH) gold particles of diameter $\mathrm{d}=64 \pm 5 \mathrm{~nm}$ and $\mathrm{d}=103 \pm 7 \mathrm{~nm}$ were obtained from the Colloid chemistry group ${ }^{3}$. University Vigo, Spain. Gold particles are reported by

\footnotetext{
${ }^{3}$ Luis M. Liz-Marzán and Isabel Pastoriza Santos (http://webs.uvigo.es/coloides/nano/main.html)
} 

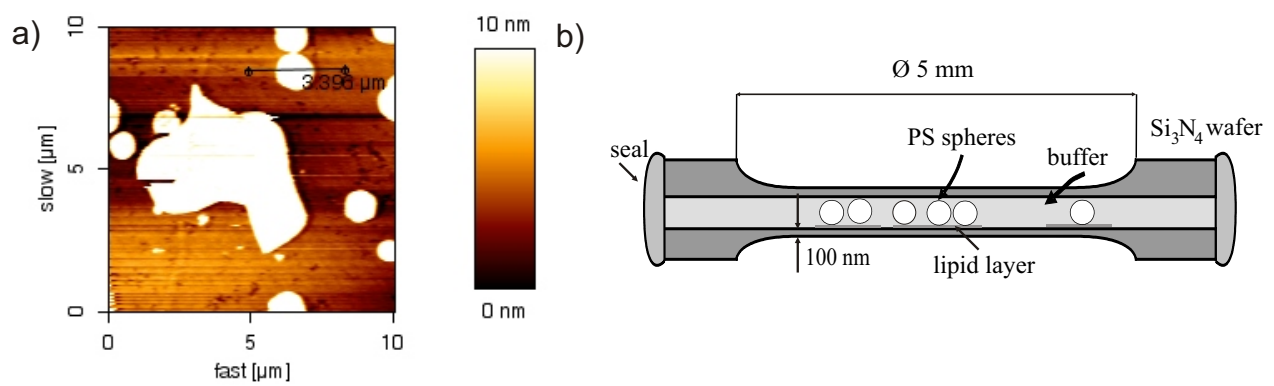

FIGURE 3.3.2. (a) The atomic force microscopy image of the incomplete DPPC/DPPS (4:1) layer, in the buffer without $\mathrm{Ca}_{2}^{+}$ions, used for future interaction with amidine polystyrene microspheres. (b) Schematic of the wet cell for scanning transmission X-ray spectro-microscopy measurements.

the producer to have a zeta potencial of $48.5 \mathrm{mV}$ and $30.8 \mathrm{mV}$, respectively. Scanning electron microscopy images of PSS/PAH gold particles are shown in Fig. 3.3.1 (b, c).

UV/visible spectroscopy: The UV/visible spectrum of PSS/PAH coated gold nanoparticle solution was recorded on a Lambda 40 (Perkin-Elmer) UV/visible spectrometer after adding 100 $\mathrm{mM} \mathrm{NaCl}$ to monitor a possible flocculation of gold particles in salt solution.

Buffer solutions: pH 4.6 was attained with $10 \mathrm{mM}$ acetic acid (HAc; Fluka, Deisenhofen, Germany) buffer and $\mathrm{pH} 7.4$ with $10 \mathrm{mM}$ HEPES/NaOH buffer. For the experiments performed at different ionic strenghts, $0 \mathrm{mM} \mathrm{NaCl}, 50 \mathrm{mM} \mathrm{NaCl}, 100 \mathrm{mM} \mathrm{NaCl}$ were added respectively.

SUVs vere prepared in respective buffers as described above and fused onto a clean Si wafer (for X-ray reflectivity measurements) or a clean Si foil (for fluorescence microscopy). PSS/PAH gold nanoparticles (size of $64 \pm 5 \mathrm{~nm}$ ) were added so that their final concentration was 0.33 $\mu \mathrm{M}\left(\sim 4.5 \times 10^{7}\right.$ particles $/ 2 \mathrm{ml}$ solution $)$, or $3.3 \mathrm{nM}\left(\sim 4.5 \times 10^{5}\right.$ particles $/ 2 \mathrm{ml}$ solution $)$. After the incubation time, unattached nanoparticles were gently washed and samples were observed by fluorescence microscopy and X-ray reflectivity methods.

\subsection{Giant unilamellar vesicles mixed with gold colloids}

Giant unilamellar vesicles (GUVs) were generated by the electroformation technique [80]. DOPC/DOPS (4:1) lipid mixture in chloroform $(1 \mathrm{mg} / \mathrm{ml})$ was prepared and droplets of $15 \mu 1$ were carefully deposited on indium tin oxide (ITO) treated glass slides (PGO, CA, USA). The film was then partially dried overnight in a vacuum oven. After partial dehydration, the two ITO slides were separated with $1 \mathrm{~mm}$ teflon spacers and sealed with paste sigillum wax (Vitrex, Copenhagen, Denmark). About $1 \mathrm{ml}$ buffer containing $10 \mathrm{mM}$ HEPES $(\mathrm{NaOH})$ with $\mathrm{pH} 7.4$ was added. The conductive glasses were connected to copper electrodes through two pieces of copper tape glued on the conductive side of two ITO slides in order to allow the application of voltage difference. For electroformation, an AC electric field provided by a pulse generator was applied for $3 \mathrm{~h}$ across the chamber and incremented every $5 \mathrm{~min}$ from $50 \mathrm{mV}$ to $1.6 \mathrm{~V}$ at $12 \mathrm{~Hz}$ frequency. Then, the $\mathrm{AC}$ frequency was lowered to $5 \mathrm{~Hz}$, and the voltage was raised to $2 \mathrm{~V}$ for $30 \mathrm{~min}$ to detach the giant vesicles from the glass slides. Solution of GUVs was mixed with PSS/PAH gold nanoparticles of diameter $64 \pm 5 \mathrm{~nm}$ so that their final concentration was $0.33 \mu \mathrm{M}\left(\sim 4.5 \times 10^{5}\right.$ particles $/ 20 \mu \mathrm{l}$ 
solution). $20 \mu \mathrm{l}$ of the sample were transferred into an observation chamber, made of a glass slide and a coverslip spaced by two strips of double-sided adhesive tape. Giant vesicles were observed by phase contrast with an inverted microscope (Axiovert 40; Carl Zeiss, Jena, Germany), equipped with a $40 \times$ objective (NA $=0.8$, Carl Zeiss, Jena, Germany) and a monochromatic camera CCD SONY (SONY Corp., USA). Phase-contrast microscopy image of DOPC/DOPS (4:1) GUV is shown in Fig. 3.5 .

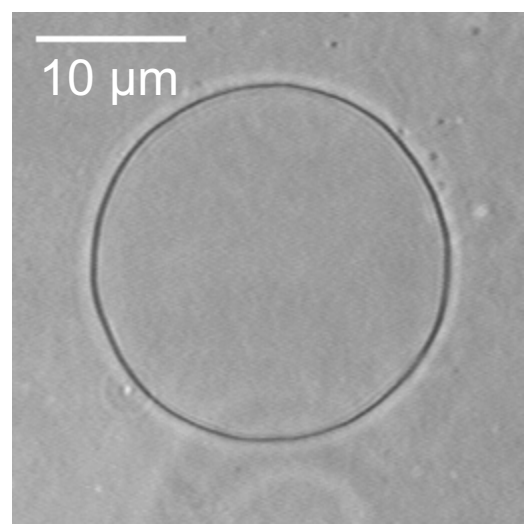

FIGURE 3.5.1. Phase-contrast microscopy image of DOPC/DOPS (4:1) giant unilamellar vesicle. The vesicle is $\sim 23 \mu \mathrm{m}$ in diameter.

\subsection{Lipid multilayers}

Lipid multilayer samples were prepared on $\mathrm{Si}_{3} \mathrm{~N}_{4}$ windows. Lipids (DOPC, DOPS, DMPC) were dissolved in chloroform, at total concentrations $5 \mathrm{mg} / \mathrm{ml}$. The drop of $1 \mu 1,0.5 \mu 1$ or $0.01 \mu 1$ was then carefully spread onto well-leveled and cleaned $\mathrm{Si}_{3} \mathrm{~N}_{4}$ windows. The spreaded solution was allowed to dry. Finally, the films were exposed to high vacuum over $24 \mathrm{~h}$ to remove all traces of solvent. The surface topography of lipid multilayers was characterized by the PL $\mu 2300$ Optical Imaging Profiler (Sensofar Tech S.L., Barcelona, Spain). Images were taken in the phase shifting interferometer modus. Sample was prepared for the near-edge X-ray absorption spectromicroscopy experiment described in chapter 6 .

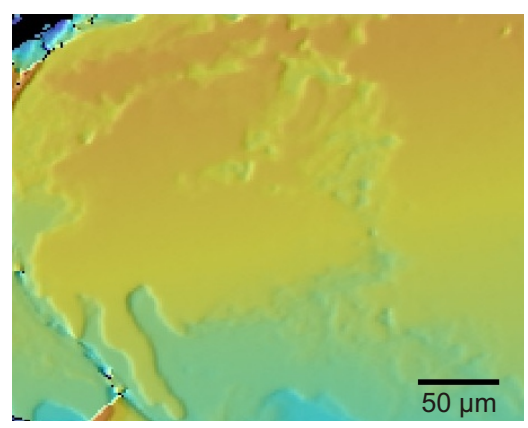

FIGURE 3.6.1. DMPC supported multilayers (of $\sim 120 \mathrm{~nm}$ in thickness) imaged in a dried state by Optical Imaging Profiler. 
Fourier transform infrared (FTIR) measurement: Transmission infrared spectra were recorded at a room temperature on a JASCO FTIR 4100 (Jasco Co., Tokyo, Japan) instrument. The FTIR spectrometer was equipped with a narrow band liquid-nitrogen-cooled mercury cadmium telluride (MCT) detector. The multilamellar DOPS stacks were deposited onto well-leveled and cleaned $\mathrm{CaF}_{2}$ substate as described above. Several stacks were prepared and hydrated with the respective buffer solution (10 $\mathrm{mM}$ acetic acid/ $\mathrm{HCl}(\mathrm{NaOH}), 50 \mathrm{mM} \mathrm{NaCl}, \mathrm{pH}$ 3.9-4.7). Prepared samples were then capped by a second $\mathrm{CaF}_{2}$ substrate and sealed with Parafilm to form a wet cell. Transmittance spectra were collected. Typically 600 interferograms were recorded with $0.5 \mathrm{~cm}^{-1}$ resolution. 


\section{CHAPTER 4}

\section{Introduction to X-ray interaction with matter, index of refraction}

$\mathrm{X}$-rays are electromagnetic waves as first demonstrated by Laue from X-ray diffraction patterns $[\mathbf{8 1}]$. X-rays can be produced by acceleration or deceleration of electrons, either in vacuum (synchrotrons), or in metallic targets (tubes). The most widely used X-rays have a typical wavelength $(\lambda)$ on the order of $0.1 \mathrm{~nm}$. This wavelength is associated with a very high frequency on the order of $10^{19} \mathrm{~Hz}$, which is at least four orders of magnitude higher than the eigen frequency of an electron bound to a nucleus.

When an electromagnetic plane wave with an electric field $\vec{E}$ is incident on a charge distribution, this oscillates in response to this driving field. This motion can be described as a series of damped harmonic oscillators. The displacement $\vec{x}$ of an electron from its equilibrium position leads to a dipole momentum $\vec{p}$ of the atom $[\overline{\mathbf{8 2}}]$

$$
\vec{p}=-e \vec{x}=\frac{e^{2}}{m_{e}} \frac{1}{\omega_{0}^{2}-\omega^{2}-i \omega \gamma} \vec{E},
$$

where $m_{e}$ is the mass of the electron, $\gamma$ the damping constant and $\omega, \omega_{0}$ are the frequency and the resonant frequency of the electromagnetic field respectively. For $n_{a}$ atoms per unit volume with $Z$ electrons per atom, the volume polarization of the material is

$$
\vec{P}=\frac{n_{a} e^{2}}{m_{e}}\left(\sum_{j} \frac{f_{j}}{\left(\omega_{j}^{2}-\omega^{2}-i \omega \gamma_{j}\right)}\right) \vec{E}
$$

The $f_{\mathrm{j}}$ terms, satisfying the requirement $\sum f_{\mathrm{j}}=Z$, are weighting factors known as oscillator strengths. They are a measure of the atomic transition probabilities. Each electron has its own resonance frequency $\omega_{j}$ and a damping coefficient $\gamma_{j}$.

The polarization is related to the electric field by the electric susceptibility $\chi_{e}$ as $\vec{P}=\varepsilon_{0} \chi_{e} \vec{E}$. From the relation between the polarization and the electric field the electric susceptibility becomes

$$
\chi_{e}=\frac{n_{a} e^{2}}{\varepsilon_{0} m_{e}}\left(\sum_{j} \frac{f_{j}}{\left(\omega_{j}^{2}-\omega^{2}-i \omega \gamma_{j}\right)}\right)=\frac{n_{a} r_{e} \lambda^{2}}{4 \pi^{2} \varepsilon_{0}}\left(\sum_{j} \frac{\omega^{2} f_{j}}{\left(\omega_{j}^{2}-\omega^{2}-i \omega \gamma_{j}\right)}\right) .
$$

Here $r_{e}=e^{2} / m_{e} c^{2}$ is the classical radius of the electron.

For X-rays, in the limit of high frequency $\left(\omega \gg \omega_{j}\right)$ and low damping $(\gamma \rightarrow 0)[\mathbf{8 3}]$, the $\lambda^{2}$ expression in Eq. 4.0.3 reduces to

$$
\chi_{e}=-\frac{n_{a} r_{e} \lambda^{2}}{4 \pi^{2} \varepsilon_{0}} \sum_{j} f_{j}=-\frac{n_{a} r_{e} \lambda^{2}}{4 \pi^{2} \varepsilon_{0}} Z
$$



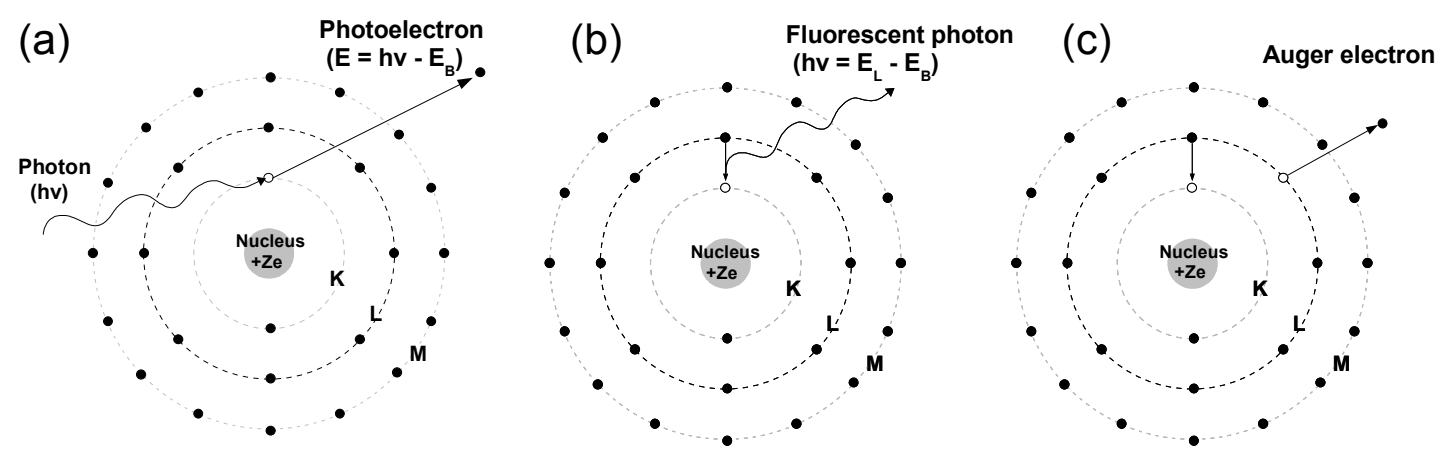

FIGURE 4.0.1. (a) Photoionization by an incident $x$-ray photon leaves the atom with a vacancy in a core shell. The energy difference is (b) released in form of a fluorescent photon, or (c) transferred to an Auger electron which is ejected from the atom. Processes are illustrated with a simplified model $[\mathbf{8 3}]$.

We can now introduce the index of refraction $n[\overline{\mathbf{8 2}}]$ in a medium with the wavevector $k=$ $2 \pi / \lambda$ as

$$
n=\frac{k}{k_{0}}=\frac{\sqrt{\mu \varepsilon}}{\sqrt{\mu_{0} \varepsilon_{0}}}
$$

where $\mu$ is a magnetic permeability, expressed as $\mu=\mu_{0}\left(1+\chi_{m}\right)$, in analogy with expression for dielectric constant $\varepsilon=\varepsilon_{0}\left(1+\chi_{e}\right)$. Inserting both equations into Eq. 4.0.5 gives

$$
n=\sqrt{\left(1+\chi_{e}\right)\left(1+\chi_{m}\right)} \simeq \sqrt{\left(1+\chi_{e}\right)} \simeq 1+\frac{1}{2} \chi_{e},
$$

neglecting the small magnetic susceptibility.

Inserting the Eq. 4.0.4 into Eq. 4.0.6 we end up with

$$
n \approx 1-\frac{1}{2} \frac{n_{a} r_{e} \lambda^{2}}{4 \pi^{2} \varepsilon_{0}} \sum f_{j}
$$

For X-rays, the index of refraction is often written as $n=1-\delta-i \beta=1-\frac{r_{e} \lambda^{2}}{2 \pi} n_{a}\left(f_{1}+i f_{2}\right)$, where

$$
\delta=\frac{r_{e} \lambda^{2}}{2 \pi} n_{a} f_{1}=\alpha \lambda^{2} f_{1}
$$

and

$$
\beta=\frac{r_{e} \lambda^{2}}{2 \pi} n_{a} f_{2}=\alpha \lambda^{2} f_{2}
$$

As a consequence, the interaction of X-rays with matter can be well described (in a classical way for a first approach) by an index of refraction which characterizes the change of direction of the X-ray beam when passing from air to a material. Values of $f_{1}$ and $f_{2}$ have been tabulated by Henke et al. [84] for all elements from hydrogen to uranium $(Z=92)$ and for photon energies extending from $50 \mathrm{eV}$ to $30 \mathrm{keV}$. 


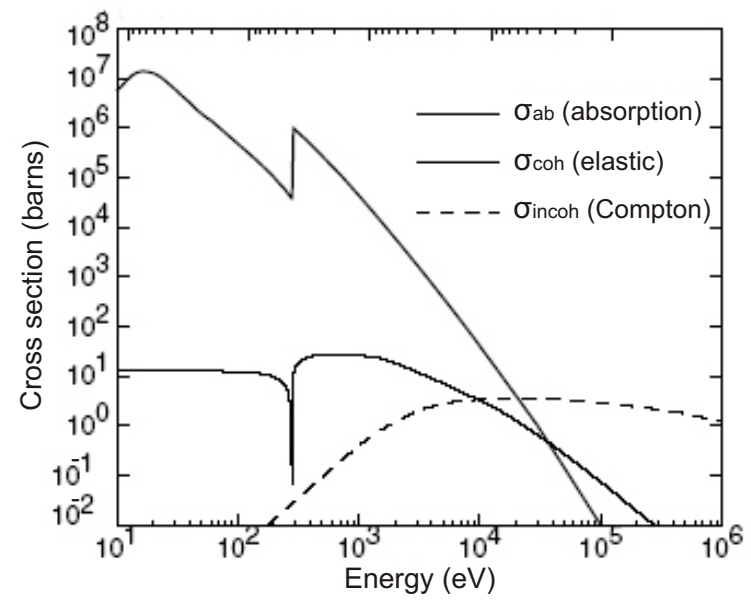

FIGURE 4.0.2. X-ray cross sections in carbon for photoelectric absorption and elastic and inelastic scattering. (Figure from Kirz et al. [85]).

The incident beam may be either absorbed, scattered elastically (coherent), or inelastically (Compton) by the atom $[\mathbf{8 3},[\mathbf{8 1}]$. The total cross section $\sigma$ describes the probability that an incident photon will interact with an atom. In the photoelectric absorption process, the X-ray is absorbed in the atom and its energy $E$ is transmitted to an electron in the $K$-shell, $L$-shell etc., with binding energy $E_{K}, E_{L}$ etc. The electron is expelled from the atom with an energy of $E-E_{K}, E-E_{L}$ etc. leaving the atom in an excited state with a core vacancy. The core vacancy can be than filled by an electron from one of the higher lying shells with concomitant fluorescent radiation (Fig. $4.0 .1(\mathrm{a}, \mathrm{b}))$. The atom can adjust to the core vacancy through the Auger process in which one electron makes a transition to the core vacancy, while a second electron of characteristic energy is emitted (Fig. 4.0.1 (c)). In the case of elastic scattering, the incident photon preserves its energy and is scattered at a new angle. Elastic scattering becomes important in the cases where scattered amplitudes can be added coherently. Compton scattering is explained as inelastic scattering of the incident photon off an electron, whereby energy and momentum are preserved between the photon and the electron. Part of the energy and momentum is transferred to the electron, and the photon is scattered off at a new angle, with reduced or enhanced photon energy and therefore increased wavelength. Fig. 4.0.2 shows the cross sections for the three interaction mechanisms listed above in the case of carbon.

It can be seen that in the soft X-ray region $(\lesssim 1 \mathrm{keV})$ Compton scattering is negligible compared to absorption and elastic scattering. At higher photon energies up to $10 \mathrm{keV}$, Compton scattering becomes a more relevant fraction of the total interaction cross section. Increasing the $\mathrm{X}$-ray energy primarily reduces photoelectric absorption and thereby minimizes radiation damage and incoherent background. However, the elastic scattering signal is reduced at higher energies as well and Compton scattering becomes the dominating interaction.

When X-rays interact with a dense medium consisting of a very large number of atoms or molecules and when the momentum transfer is small with respect to the inverse of the interatomic 
distance, it is sometimes more convenient to treat the material as a continuum with an interface to the surrounding medium. At the interface the X-ray beam is refracted and reflected [81] . These processes will be described in the Chapter 5 . 


\section{CHAPTER 5}

\section{X-ray reflectivity study of solid supported lipid membranes}

\subsection{Introduction}

Recent studies of solid supported lipid bilayers have demonstrated the potential of X-ray reflectivity to probe the molecular structure of lipid model membranes [61, 86]. The density profile across a single bilayer $\rho(z)$ in the fluid state can be retrieved on an absolute scale under physiologically relevant conditions of excess water or buffer solutions. Such systems serve as simple model systems for the much more complex biological counterparts [87] and are also interesting components for biotechnological applications, for example as bio-functionalized solid surfaces.

Here we use X-ray reflectivity to probe the structure of two-component lipid mixtures, with varied surface charges, both in the fluid and the gel phases. Protocols for the deposition of solid supported lipid bilayers by vesicle fusion go back to pioneering work by McConnel [41], and have also been adapted for deposition of charged bilayers [49, 45]. Cationic and mixed bilayers on solid surfaces have been used to study the absorption of biomolecules (such as DNA) with a membrane mainly by fluorescence microscopy $[\overline{\mathbf{8 8}}]$. The lipid molecules self-assemble into bilayers in the presence of a hydrophilic solid support, which stabilizes a thin water layer between the support and the bottom leaflet of the bilayer.

In this chapter we investigate the effect of the bilayer charge on the water spacing between bilayer and silicon substrate, as well as detailed structural characteristics of mixed two-component bilayers. Structural parameters, such as membrane thickness, area per lipid, water thickness, and the number of water molecules per lipid were calculated directly from an electron density profile of the model bilayers. For this purpose, the reflectivity signal above background has been measured up to $q_{z} \simeq 0.6 \AA^{-1}$ in the fluid phase and $q_{z} \simeq 0.7 \AA^{-1}$ in the gel phase of the bilayers, using synchrotron radiation, while a range of at least $0.3 \AA^{-1}$ and $0.35 \AA^{-1}$ was exploitable in-house. The dynamic range covered in the reflectivity signal was seven orders of magnitude for the inhouse sealed tube measurements, and more than eight orders of magnitude at the synchrotron. The bilayer was parameterized by an adaptable number $n$ of Fourier coefficients [89], depending on the $q_{z}$-range probed. Note, that reflectivity yields the bilayer scattering density profile $\rho(z)$ on an absolute scale without free scaling parameters. The parameters were optimized by a global search using a genetic optimization algorithm (Volker Türck, Optimize 5.0).

Phase separation and lateral demixing of lipids in model systems has presently attracted increased intention again, in view of the possible functionality in biological membranes associated with lateral heterogeneity. Structural characterization on molecular scale, capable to probe lipid segregation on the nanometer scale, is however challenging. Here we show that a splitting of the 
density profile in the headgroup region of mixed bilayers is observed, which we associate with lateral demixing of the two lipid components. We also show that the water layer $d_{w}$ between silicon and the headgroup of the lower leaflet can be determined with high precision and is found to vary with bilayer charge density $\sigma$.

A further, more technical motivation for the present study was to demonstrate the feasibility of single membrane reflectivity experiments with sealed tube in-house instrumentation. Recent work on X-ray reflectivity from solid-supported bilayers has exclusively concentrated on synchrotron experiments $[\mathbf{6 1}, \mathbf{8 6}]$. In this work we show that high resolution density profiles obtained from least-square fitting to a parameterized bilayer model can be obtained also with an in-house reflectivity setup using $M o K_{\alpha}$ radiation to penetrate the excess water above the bilayer. Note that in-house experiments are accessible to a broader research community. Furthermore, beam damage does not pose any problems, e.g., in contrast to intense undulator radiation. Finally, complicated and lengthy measurements, in particular addressing lipid-protein interaction and/or complex model systems, can be sometimes more easily be addressed in in-house experiments where the beam can be used over several weeks in a row.

\subsection{X-ray reflectivity: theory, experiment, and analysis}

Specular reflection of X-rays from a plane surface can yield information about its electronic structure in a perpendicular direction $[\mathbf{8 1}]$. X-ray reflectivity of surfactant films was first demonstrated by Pershan and Als-Nielsen [00].

5.2.1. Critical angle of reflection. For X-rays, the refractive index $n$ (derived in Chapter 4) of a material is slightly less than unity $[\mathbf{8 1}]$. Passing from air $(n=1)$ to the reflecting material $(n<1)$, it is possible to totally reflect the beam if the incident angle $\alpha_{i}$ (i.e. the angle between the surface of the sample and the incident beam) is small enough. It is known as the total external reflection of X-rays. For this to occur, the incident angle must be smaller than the critical angle $\alpha_{c}$ defined as

$$
\cos \alpha_{c}=n=1-\delta
$$

Since $n$ is very close to unity, this angle is very small and the Taylor approximation in $\alpha_{c}$ yields

$$
\begin{gathered}
\alpha_{c}^{2}=2 \delta=\frac{r_{e} \lambda^{2}}{\pi} \rho \\
\cos \theta_{i}=\cos \theta_{c}=1-\delta \\
1-\frac{\theta_{c}^{2}}{2 !}+\ldots=1-\delta \\
\theta_{c} \approx \sqrt{2 \delta}=\lambda \sqrt{r_{e} \rho(r) / \pi}
\end{gathered}
$$

where $r_{e}$ is the classical electron radius and $\rho(r)$ is the electron density.

The corresponding critical wave vector $q_{c}$ is then

$$
q_{c}=4 \sqrt{\rho(r) r_{e} \pi}
$$




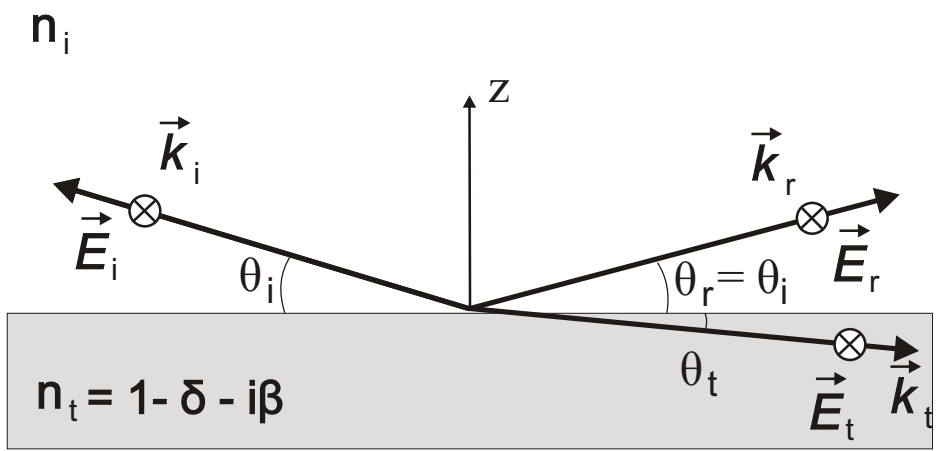

FIGURE 5.2.1. A plane electromagnetic wave with wavevector $\overrightarrow{k_{i}}$ hits a surface at grazing angle $\theta_{i}$. The wave splits into a reflected $\left(\theta_{i}=\theta_{r}\right)$ and a refracted wave transmitted at the angle $\theta_{t}$.

5.2.1.1. Reflected intensity from ideally flat surface. Now the reflectivity of a single perfectly smooth vacuum/medium interface will be calculated. The situation is shown in Fig. 5.2.1. A plane wave in vacuum, $\vec{E}_{i}(r)=\left(0, a_{i}, 0\right) \exp \left(i \vec{k}_{i} \vec{r}\right)$ with wavevector $\vec{k}_{i}=k\left(\cos \theta_{i}, 0,-\sin \theta_{i}\right)$, hits at a grazing angle $\theta_{i}$ a flat surface or a medium with the refractive index $n_{t}$. The reflected and transmitted fields can be described by $\vec{E}_{r}(r)=\left(0, a_{r}, 0\right) \exp \left(i \vec{k}_{r} \vec{r}\right)$, with $\vec{k}_{r}=k\left(\cos \theta_{i}, 0, \sin \theta_{i}\right)$, and $\vec{E}_{t}(r)=\left(0, a_{t}, 0\right) \exp \left(i \overrightarrow{k_{t}} \vec{r}\right)$, with $\vec{k}_{t}=\left(k_{t, x}, 0, k_{t, z}\right)$.

The reflectivity intensity $R$ is defined as the product of the amplitude reflectivity $r$ with its conjugate complex $r^{*}$ expressed by the ratio of incident and reflected amplitude $a_{i} / a_{r}$ :

$$
R=\left|a_{i} / a_{r}\right|^{2}=r r^{*}
$$

The incident, reflected and transmitted waves show for the amplitudes at $z=0$

$$
\begin{gathered}
a_{i}+a_{r}=a_{t} \\
a_{i} k_{i}+a_{r} k_{r}=a_{t} k_{t}
\end{gathered}
$$

with $k=\left|k_{i}\right|=\left|k_{r}\right|$ and $n k=k_{t}$ follows

$$
\begin{gathered}
a_{t} n k \cos \theta_{t}=a_{i} k \cos \theta_{i}+a_{r} k \cos \theta_{r} \\
n a_{t} k \sin \theta_{t}=\left(a_{i}-a_{r}\right) k \sin \theta_{i} \\
n=\frac{\left(a_{i}-a_{r}\right) k \sin \theta_{i}}{\left(a_{i}+a_{r}\right) k \sin \theta_{t}} \\
n \frac{\sin \theta_{t}}{\sin \theta_{i}}=\frac{\left(a_{i}-a_{r}\right)}{\left(a_{i}+a_{r}\right)} \approx \frac{\theta_{r}}{\theta_{i}}
\end{gathered}
$$

Finally,

$$
r \equiv \frac{a_{r}}{a_{i}}=\frac{\theta_{i}-\theta_{r}}{\theta_{i}+\theta_{r}}
$$

is the Fresnel equation of reflectivity and

$$
t \equiv \frac{a_{t}}{a_{i}}=\frac{2 \theta_{i}}{\theta_{i}+\theta_{r}}
$$

is the Fresnel equation of transmittivity. The resulting reflectivity intensity then is 


$$
R_{F}=r r^{*}=\left(\frac{\theta_{i}-\theta_{r}}{\theta_{i}+\theta_{r}}\right)^{2}
$$

With $q_{z}=4 \pi / \lambda \sin \theta_{r}=2 k \sin \theta_{r} \approx 2 k \theta_{r}$ it can be rewritten as:

$$
R_{F}=r r^{*}=\left(\frac{q_{i}-q_{t}}{q_{i}+q_{t}}\right)^{2}
$$

Subsequently using $q_{t}^{2}=q_{i}^{2}-q_{c}^{2}$

$$
R_{F}=r r^{*}=\left(\frac{q_{i}-\sqrt{q_{i}^{2}-q_{c}^{2}}}{q_{i}+\sqrt{q_{i}^{2}-q_{c}^{2}}}\right)^{2}=\left(\frac{1-\sqrt{q_{i}^{2}-q_{c}^{2}} / q_{i}}{1+\sqrt{q_{i}^{2}-q_{c}^{2}} / q_{i}}\right)^{2}=\left(\frac{1-\sqrt{1-q_{c}^{2} / q_{i}^{2}}}{1+\sqrt{1-q_{c}^{2} / q_{i}^{2}}}\right)^{2}
$$

For $q_{i}^{2}>q_{c}^{2}$ the reflectivity results in

$$
R_{F} \approx\left(\frac{q_{c}}{q_{i}}\right)^{4}
$$

Figure 5.2.2 (left) shows the Fresnel reflectivity as a function of the angle of incidence $\theta_{i}$ normalized by the critical angle $\theta_{c}$ of a silicon/vacuum interface $\left(\theta_{c}=0.22^{\circ}\right)$ for the $\lambda=1.54 \AA$ and different absorption/dispersion ratios $(\beta / \delta)$. The absorption only plays a role in the vicinity of the critical angle, leading to a "rounding" in this region, and is negligible for larger angles of $\theta_{i}$. The function $R_{F}$ decreases rapidly for incident angles $\theta_{i}>\theta_{c}$ (see Eq. 5.2.7). Figure 5.2.2 (right) shows the Fresnel transmission again as a function of the angle of incidence $\theta_{i}$ normalized by the critical angle $\theta_{c}$ of a silicon/vacuum interface $\left(\theta_{c}=0.22^{\circ}\right)$ for the $\lambda=1.54 \AA$ and different $\beta / \delta$ ratios. For $\theta_{i} \approx \theta_{c}$ this function reaches a maximum. This is due to the fact, that the reflected and the transmitted waves interfere constructively, thus enhancing the amplitude of the transmitted wave by a factor of two $[\overline{\mathbf{9 0}}]$. For larger angles $\theta_{i}$ the Fresnel transmission tends to unity.

5.2.2. Kinematical theory. The kinematical theory or first Born approximation simplifies the expression for the intensity of specular reflectivity for non-ideal surfaces that are either rough or exhibit structure. The basic assumption of the Born approximation is that the amplitude of the transmitted wave is equal to that of the incident wave [90, 82]. This amounts to neglecting refraction effects. The kinematical approach is valid in the so-called weak scattering regime, i.e. when the cross section for the scattered radiation is small and therefore multiple scattering effects may be neglected. This approximation breaks down in the region of the critical angle, while it is valid for incident angles of, say $\theta_{i}>3 \theta_{c}[\mathbf{9 0}]$.

Within the first Born approximation, the X-ray reflectivity signal is a direct measure of the average electron density of the sample, perpendicular to the surface normal $z, \rho(z)$. More specifically, the ratio of the X-ray reflectivity to the Fresnel reflectivity will deviate from unity by the absolute value of the square of amplitude of a surface structure factor $F\left(q_{z}\right)$. The structure factor 

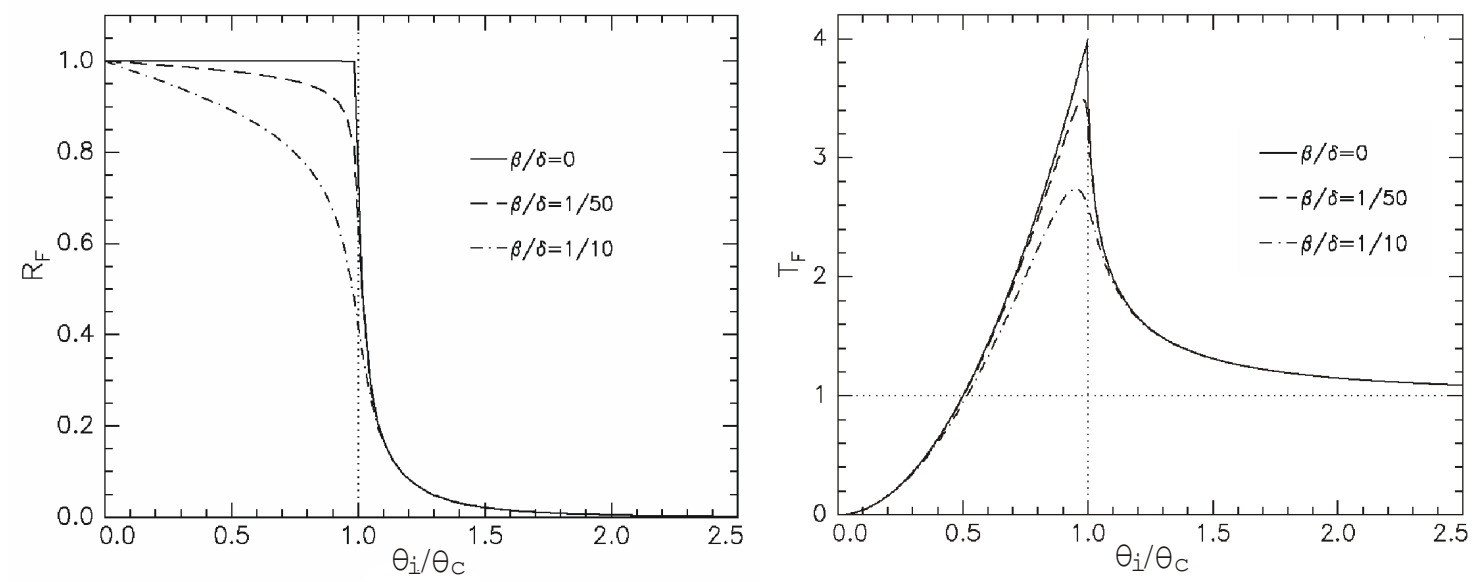

FIGURE 5.2.2. Fresnel reflectivity $R_{F}$ (left) and Fresnel transmission $T_{F}$ (right) vs. the angle of incidence $\theta_{i}$ normalized by the critical angle $\theta_{c}$ of a silicon/vacuum interface $\left(\theta_{c}=0.22^{\circ}\right)$ for the $\lambda=1.54 \AA$ and different absorption/dispersion ratios $\beta / \delta$. The absorption is only of importance in the region of the critical angle (left). The enhanced transmission for $\theta_{i} \approx \theta_{c}$ can be seen (right). (Figure from Tolan [90]).

is defined as the Fourier transform of the derivative of the in-plane average of the electron density along the surface normal $\rho_{z}[\mathbf{8 1}]$ :

$$
R(q)=R_{F}\left(q_{z}\right)\left|\frac{1}{\rho_{\infty}} \int \frac{\rho(z)}{d z} e^{i q_{z} z} d z\right|^{2}=R_{F}\left(q_{z}\right)\left|F\left(q_{z}\right)\right|^{2},
$$

where $\rho_{\infty}$ is taken to be the electron density of the bulk, $\rho(z)$ the electron density profile along $z$, $R_{F}(q)$ the Fresnel reflectivity and $F\left(q_{z}\right)$ is the structure factor. The equation 5.2.8 is known as the Master formula.

5.2.3. Rough surfaces and interfaces. Usually an interface is not ideally sharp and the reflectivity is damped by diffuse scattering due to surface roughness expressed as

$$
R(q)=R_{F}(q) e^{-q^{2} \sigma^{2}}
$$

The damping of the diffuse scattering is the Fourier transform of the derivative of the error function $f(z)=\operatorname{erf}\left(\frac{z}{\sqrt{2 \sigma}}\right)$, which is a Gaussian. The error function describes the density distribution at a rough interface. $\sigma$ is the interface rms-roughness. The scattering of the uncorrelated surface is confined to the specular direction.

5.2.4. X-ray reflectivity from a single lipid membrane. In the case of a flat substrate, covered homogeneously by a lipid bilayer, incident waves may be reflected by the interface waterbilayer or bilayer-substrate. At distances far away from the sample, the reflectivity spectra exhibits interference effects, caused by superposition of different waves reaching the detector. If the additional flight path, traveled by the wave scattered at the bilayer/substrate interface, is a multiple of 
the X-ray wavelength, constructive interference occurs. Otherwise, if the path equals an uneven multiple of half the wavelength, the waves interfere destructively and the signal completely vanishes. Usually, full destructive interference is not observed, since the interfaces always exhibit a certain roughness. These oscillations in the reflectivity spectra are the so-called Kiessig fringes [91]. Kiessig fringes indicate that the film is very smooth and homogeneous.

5.2.5. Experimental Setup. Sealed $X$-ray tubes $[\mathbf{8 1}]$ are the most widely used $x$-ray sources. The characteristic radiation is created whenever the energy of incident electrons is large enough to excite a $K$ electron of the target material. In-house laboratory experiments always suffer from the insufficient high flux of the radiation produced by X-ray tubes. Because the radiation is emitted into the whole upper half-space of the tube, the photon flux toward a particular direction is relatively low. This problem cannot be overcome using flat monochromators or collimators but it can be partially solved using a parabolically bent multilayer Göbel mirror [92]. This mirror increases the intensity at the sample site by a factor of ten compared to a beam which is conventionally defined by a collimation line of two slits. The Göbel mirror attached to a sealed tube yields an intensity at the sample site that is comparable with that of a slit arrangement and a rotation-anode tube.

Storage ring facilities provide the most intensive $\mathrm{X}$-rays, i.e. the synchrotron radiation. Extensive reviews has been published on the properties and application of synchrotron radiation [81, 93]. Synchrotron radiation facilities supply a nearly parallel and highly intense radiation beam. Here the problem of designing an X-ray reflectivity experiment is related to a proper choice of the energy and an angular resolution.

$\mathrm{X}$-ray reflectivity experiments in this work were performed both at the ID01 undulator beamline (ESRF, Grenoble), using $13.46 \mathrm{keV}$ photon energy, and at a sealed X-ray tube (D8 Advance, Bruker, Germany), operating with $\operatorname{Mo} K_{\alpha}$ radiation $(E=17.48 \mathrm{keV}, \lambda=0.0709 \mathrm{~nm})$. The in-house reflectometer was equipped with a collimating Göbel mirror system, automatic filter settings, a fast scintillation counter and the NaI-Detector which has a dynamic range up to about $2.5 \times 10^{5} \mathrm{cps}$. The beam geometry of the instrument is shown in Figure 5.2.3. The beam size in the reflectivity plane was defined by entrance slits to $50 \mu \mathrm{m}$. The collimating Göbel mirrors, $50 \mu \mathrm{m}$ beam size in the scattering plane, large divergence in the horizontal plane (due to small source-sample distance) optimizes signal to noise ratio.

The design of the ID01 beamline is described in detail in [94]. A schematic of the setup, as it was used during the experiments, is shown in Figure 5.2.3. The polychromatic X-ray beam was monochromatized by a double crystal $\mathrm{Si}(111)$ monochromator $(\mathrm{M})$ with an energy resolution of $1 \mathrm{eV}$. A computer controlled set of attenuators (A) in front of the sample was used. Then, the beam passes through a slit (S1), which is used to determine the size of the X-ray beam $(100 \mathrm{x}$ $100 \mu \mathrm{m}$ ) in front of the sample. The beam is then passed through a ion chamber (I) to measure the incident flux, and subsequently the beam impacts the sample surface (S). After reflection from the sample surface, the X-ray beam passes through two slits (S1, S2) in order to enter the X-ray detector (D). The two slits between the detector and the sample surface are used in order to define the solid angle of acceptance of the detector. As a detector, a Cyberstar scintillation point detector 


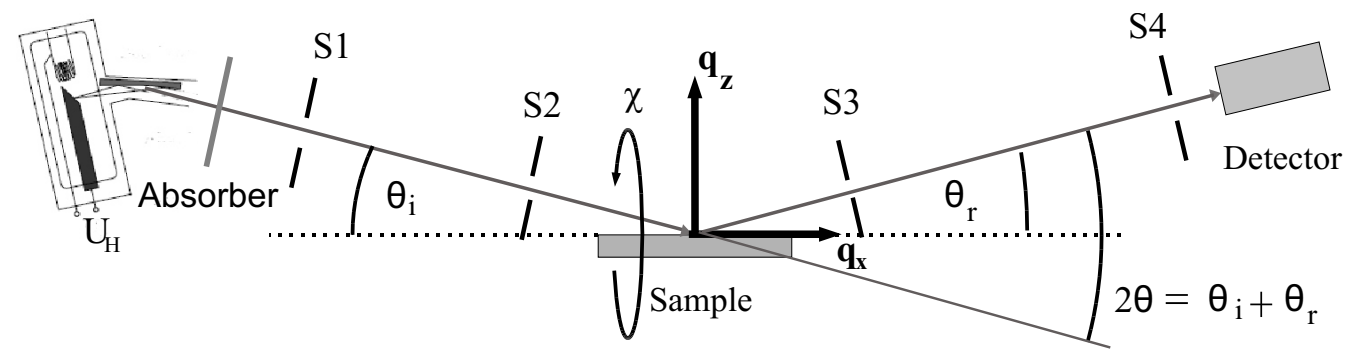

FIGURE 5.2.3. Schematic of the D8 beam geometry: A sealed X-ray tube generates $\operatorname{Mo} K_{\alpha}$ radiation, which is collimated by a Göbel mirror system. Fixed slits ( $\mathrm{S} 1$ and S2) are used to adjust the vertical angular divergence of the beam, before it hits the sample. For collimation of the reflected beam another system of two slits (S3 and S4) is used. The sample can be moved in the vertical z-direction and along the angular direction around the optical axis.

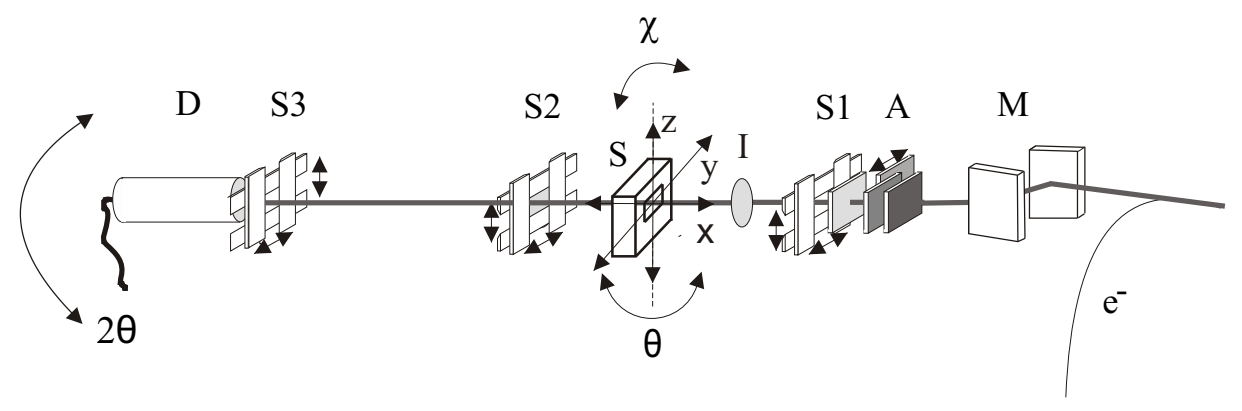

FIGURE 5.2.4. Setup at the ID01 beamline (ESRF).

was used (Oxford Danfysik, Oxford, UK). The ID01 beamline is set up as a $\theta / 2 \theta$ diffractometer, where the incoming beam has a fixed direction and the sample is moved, so that the beam hits the substrate surface at an angle $\theta_{i}$. In specular condition the detector is then moved at the same time by an angle $2 \theta_{i}$ with respect to the optical axis, so that the incoming and reflected beam obey the specular condition $\theta_{i}=\theta_{r}$.

Fig. 5.2.5 shows the picture of the measurement chamber $\left(42 \times 32 \times 10 \mathrm{~mm}^{3}\right)$, designed for this reflectivity measurement. The chamber frame is made of Teflon that is chemically inert and easy to clean. The X-ray beam enters and exits the chamber through Kapton windows. Cleaned silicon wafers with dimensions $15 \times 10 \mathrm{~mm}^{2}$ were placed into a chamber. The temperature $23.8^{\circ} \mathrm{C}$ of the chamber was kept by a flow of 1:2 glycol:water mixture from a temperature-controlled reservoir (Julabo, Germany).

At the undulator beamline ID01, at an energy $13.46 \mathrm{keV}$, we could show that the intensity loss due to beam attenuation within the liquid-filled chamber is $\sim 40 \%$. Figure 5.2.6 shows the primary beam intensity at the ID01 beamline (a) before and (b) after inserting the liquid-filled chamber.

5.2.5.1. X-ray reflectivity measurement. The impinging X-rays enclose an angle $\theta_{i}$ with the surface and have a wavevector $\overrightarrow{k_{i}}$. When the detector encloses an angle $\theta_{r}$ with the surface, 


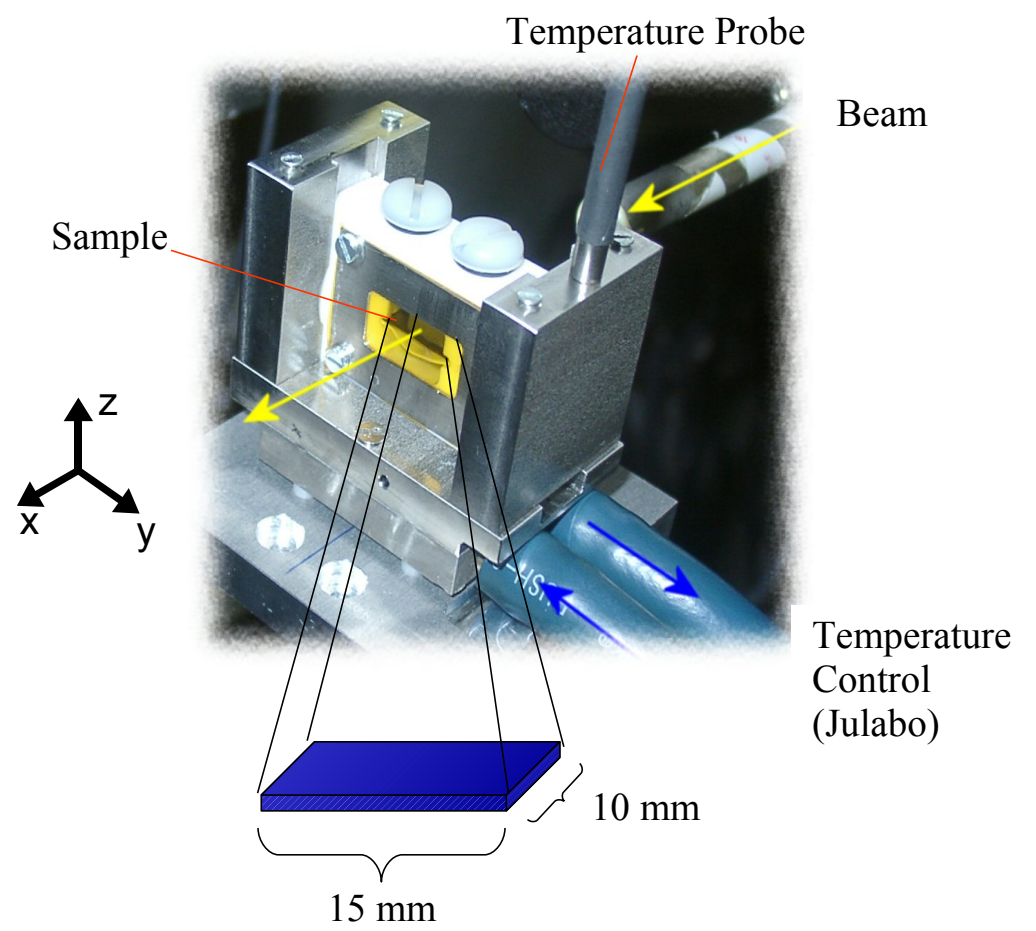

FIGURE 5.2.5. Image of the measurement cell mounted at the goniometer.
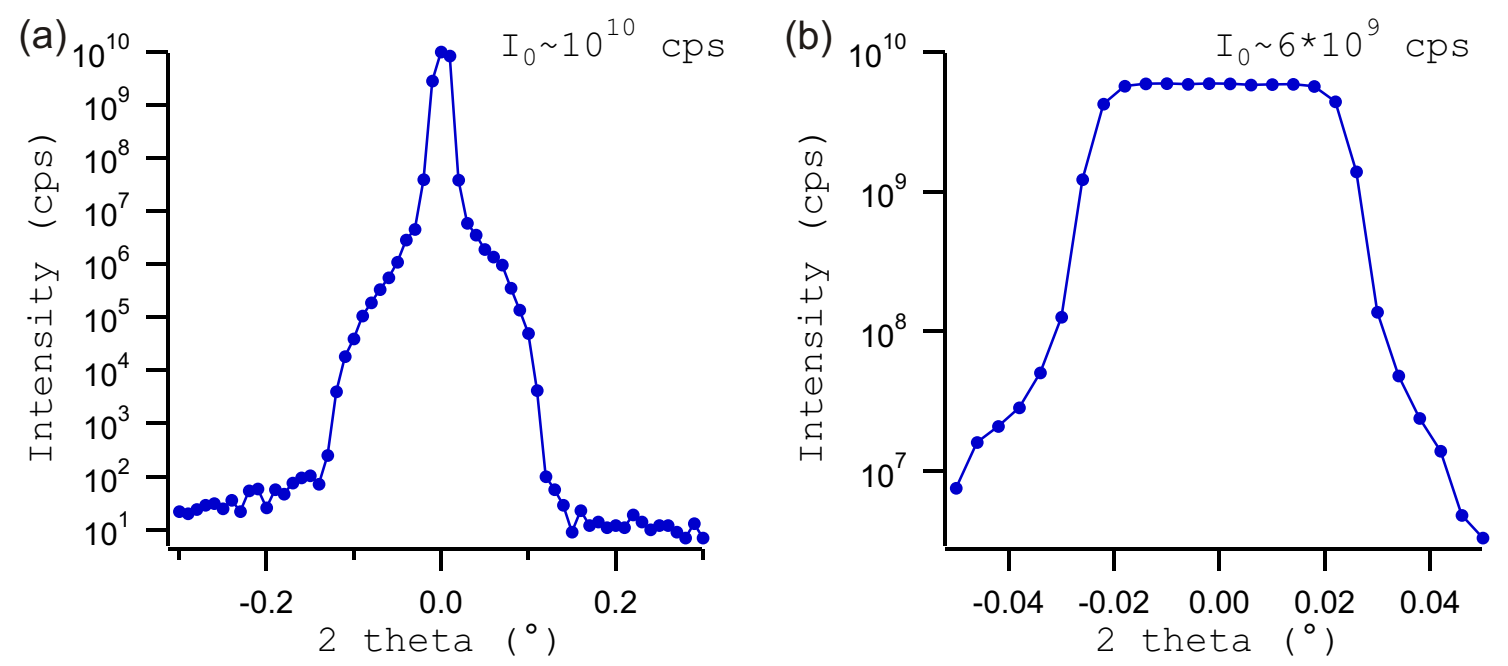

FIGURE 5.2.6. (a) The primary beam profile at the ID01 beamline and (b) the intensity loss due to beam attenuation within the liquid-filled chamber 5.2.5 $(200 \times$ $200 \mu \mathrm{m}$ slit setting).

scattered photons with the momentum $\hbar \overrightarrow{k_{r}}$ are probed. The components $q_{x}, q_{y} q_{z}$ of the wavevector transfer $\vec{q}=\overrightarrow{k_{r}}-\overrightarrow{k_{i}}$ are given by

$$
q_{x}=\frac{2 \pi}{\lambda}\left(\cos \theta_{r} \cos \phi-\cos \theta_{i}\right)
$$




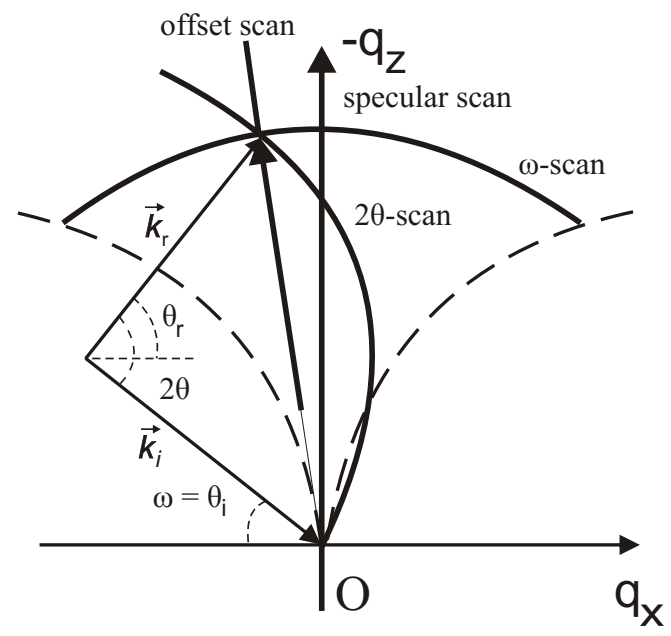

FIGURE 5.2.7. Drawing of the in plane scattering geometry (the wavevector of the incident and scattered x-rays are $\overrightarrow{k_{i}}$ and $\overrightarrow{k_{r}}$, with the incident and exit angles $\theta_{i}$ and $\theta_{r}$ respectively, and the scattering angle $2 \theta$ ), and the scans in the reciprocal plane $\left(q_{x}, q_{z}\right)$ : specular scan, offset scan, detector scan $(2 \theta$-scan) and rocking scan $(\omega-$ scan $)$. The region below the dashed lines is inaccessible for in-plane scattering experiments $\left(\theta_{i}<0\right.$ or $\left.\theta_{f}<0\right)$.

$$
\begin{gathered}
q_{y}=\frac{2 \pi}{\lambda} \cos \theta_{i} \sin \phi \\
q_{z}=\frac{2 \pi}{\lambda}\left(\sin \theta_{i}+\sin \theta_{r}\right)
\end{gathered}
$$

Figure 5.2.7 shows some typical scans in reciprocal space.

Rocking scan ( $\omega$-scan). The scattering angle $2 \theta=\theta_{i}+\theta_{r}$ remains constant and $\omega=\theta_{i}-\theta_{r}$ is changed within the limits $2 \theta / 2$. This corresponds to a rotation of the sample at fixed X-ray source and detector positions. For small $q_{z}$ the scan runs approximately parallel to the $q_{x}$ axis.

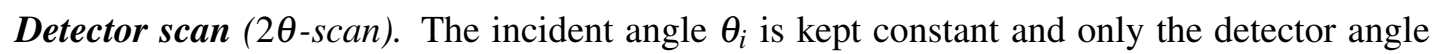
$\theta_{r}$ is changed. The scan contains information about the lateral and the vertical structure of the sample.

Specular scan. $\theta_{i}$ and $\theta_{r}$ are changed simultaneously such that the specular condition $\theta_{i}=$ $\theta_{r}=2 \theta / 2$ holds. The scan runs along the $q_{z}$ axis, i.e. $q_{x}=0$.

Offset scan (longitudinal diffuse scan). $\theta_{i}$ and $\theta_{r}$ vary simultaneously so that the condition $\theta_{i}=2 \theta / 2+\delta \theta_{i}$ holds. The scan runs on a line at an angle $\delta \theta_{i}$ with respect to the $q_{z}$ axis. $\delta \theta_{i}$ should be greater than the width of the specular peak and is typically on the order of a few hundredth of a degree.

5.2.6. Radiation damage and reproducibility. In the full synchrotron beam, radiation damage was observed. The high brilliance of synchrotron source is commonly reported to cause major damage in biological samples [95]. A main reason for this damage is the creation of photoelectrons in the (silicon) substrate, which ionize atoms in the biological or organic sample on top of it, so that chemical reactions are induced that destroy the sample structure. We have detected a systematic shift of the minima position, shown in Figure 5.2.8, by measuring a reflectivity around the second minima $\left(1.3<q_{z}<1.6\right)$ with increased illumination time (exposure time was $0.5 \mathrm{sec}$ ). 


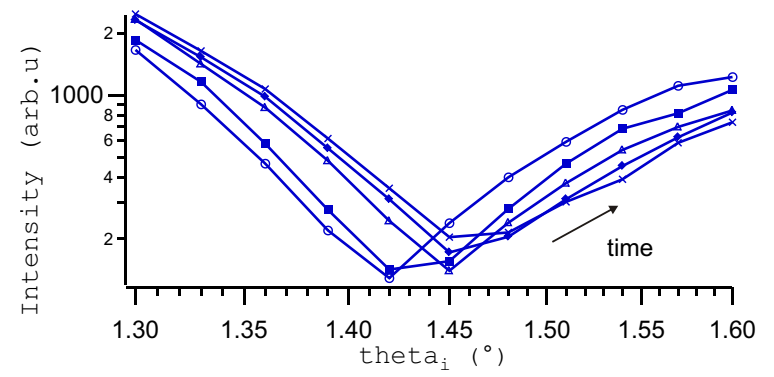

FIGURE 5.2.8. The reflectivity curves in the region around the second minima with increasing exposure time using the full beam with no attenuators. The shift in position of the minima as a function of illumination time (exposure time was $0.5 \mathrm{sec}$ ) was already reported in [86]. This effect can be ascribed to radiation damage, most likely originating from free radical generation by photoelectrons at the solid surface.

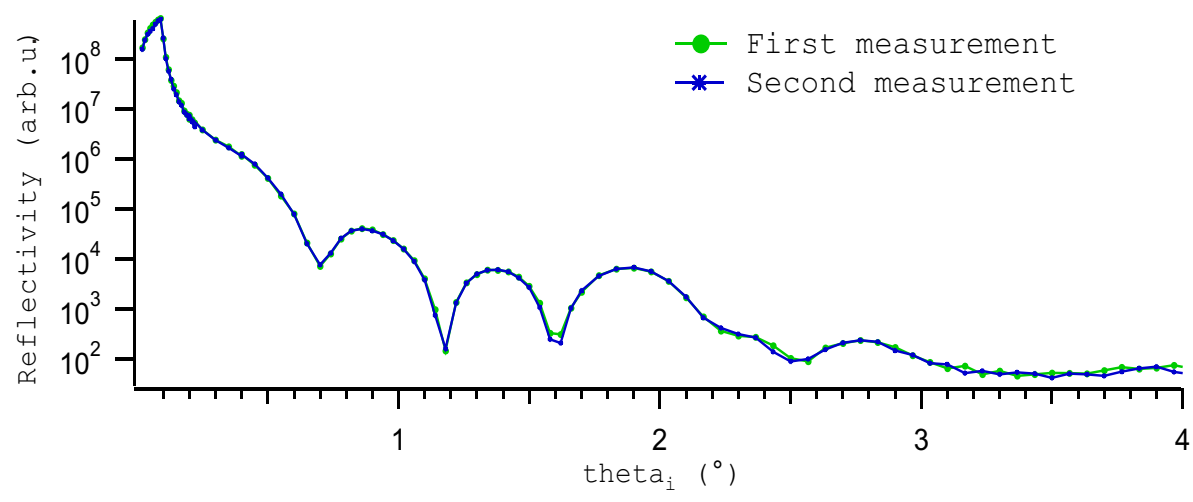

FIGURE 5.2.9. Two reflectivity measurements of the same sample (DPPC/DPPS $(4: 1))$, measured with the same scanning configurations at two different positions of the sample.

To achieve data sets unspoiled by radiation damage, the following measures were combined: (i) A computer controlled set of attenuators in front of the sample was used, see Figure 5.2.11 (a), limiting the full beam exposure only to a selected number of points in the high $q_{z}$-range. (ii) The sample was translated during illumination in $y$-direction, perpendicularly to the direction of the incident beam and data were always collected from undamaged areas. (iii) A fast shutter system was installed to minimize the exposure time during motor movements. Reproducibility of the measured data was then checked along various parts of the reflectivity curve (Fig. 5.2.9. The scans were completed in approximately $30 \mathrm{~min}$. For reflectivity measurements carried out at D8 Advance reflectometer, automatic attenuator settings were used. Radiation damage was not observed. The scans were completed in approximately $6 \mathrm{~h}$.

5.2.7. Data Treatment. $X$-ray scattering and particularly raw reflectivity data cannot be directly compared with model calculation for two reasons: (i) In this particular experiment the specular reflectivity is considered the data with the diffuse reflectivity being the background. (ii) For very small incident angles the sample surface is almost parallel to the incident beam and parts 
of the incoming radiation do not hit the sample. This leads to a reduction of the reflected intensity and the measured reflectivity becomes less than unity in the region of total external reflection. The reflectivity curves were corrected for background (diffuse) scattering, illumination and normalized to primary beam intensity, measured by photodiode monitor.

Processing the raw data entails four main steps:

- Since the measured curve consists of several parts, these must first be added together so that a continuous curve forms. The scans are corrected for attenuation and normalized by the monitor signal.

- The measured intensity is plotted as a function of the momentum transfer $q_{z}$. The momentum transfer perpendicular to the interface is defined as a function of the incident angle $\theta_{i}$,

$$
q_{z}=\frac{4 \pi}{\lambda} \sin \left(\theta_{i}\right)
$$

- Next, the background is removed from the data. Background removal consists of simply subtracting the diffuse reflectivity from the specular reflectivity.

At this point the counting-statistics has to be considered. The measured quantity is not the scattered intensity $I$ itself, but a discrete number of photons $N$ detected by detector within a time $t$. Thus, $N$ is Poisson distributed with an error $\Delta N=\sqrt{N}$. For sufficiently large $N$, a normal distribution with $\sigma=\Delta N$ is assumed [96]. If $N$ counts have been measured in a time $t$, the intensity is equal to $I=N / t$ with an error $\Delta I=\sqrt{N} / t$. After the background substraction the error propagates as $\Delta I_{\text {corrected }}=\sqrt{\Delta I^{2}+\Delta I_{\text {bgr }}^{2}}$.

- The remaining step in the data processing is to correct the data for the sample footprint. For very small incident angles the sample surface is almost parallel to the incident beam and parts of the incoming radiation do not hit the sample. This leads to a reduction of the reflected intensity and the measured reflectivity becomes less than unity in the region of total external reflection. If a rectangular shaped beam of width $h$ and a sample of length $l$ is assumed (Fig. 5.2.10), the angle of complete irradiation of the sample is given by $\theta_{g}=\arcsin (h / l)$ and the reflected intensity is reduced by a factor $\beta=\sin \theta_{i} / \sin \theta_{g}$ for $\theta_{i} \leq \theta_{g} ;\left(\right.$ for $\left.\theta_{i}>\theta_{g}, \beta=1\right)$.

- Finally, the corrected specular reflectivity is normalized to primary beam intensity, measured by photodiode monitor. The data can be divided by the Fresnel reflectivity of the bare silicon substrate to obtain the signal coming from the bilayer itself.

X-ray reflectivity scan before and after correction are shown in Fig. 5.2.11. Igor Pro (Wavemetrics, Portland, USA) procedures for data correction are listed in Appendix B. Once the data is in this format it is possible to compare it with theory by fitting to find structural parameters. 


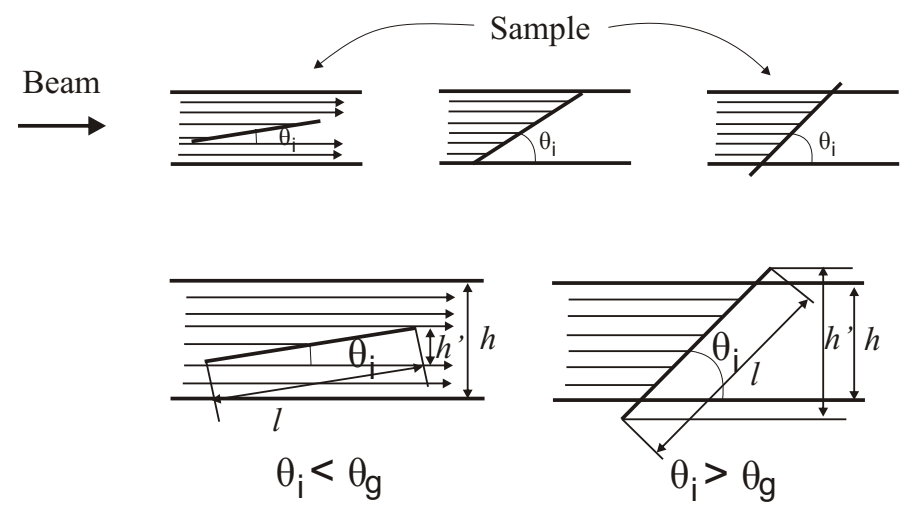

FIGURE 5.2.10. The dependence of the illumination of the sample (length $l$ ) on the incident $\theta_{i}$ : For the incident angles below the cover angle $\theta_{g}\left(\theta \leq \theta_{g}\right)$ larger beam size $h$ increases the integrated primary intensity and only a part of the primary beam is reflected. While for $\theta_{i}>\theta_{g}$, the whole beam is reflected.

\subsection{Semi-kinematical model for lipid bilayer reflectivity}

The method, we used for data analysis, is based on semi-kinematical approximation 11 of the reflectivity. The X-ray reflectivity from an interface is characterized by the electron density profile $\rho(z)$ between a medium 1 with electron density $\rho_{1}$ and a medium 2 with density $\rho_{2}$ and can be expressed by the so-called master formula $[\mathbf{8 1}]$ :

$$
R\left(q_{z}\right)=R_{F}\left(q_{z}\right)\left|\frac{1}{\Delta \rho_{12}} \int \frac{d \rho(z)}{d z} e^{i q_{z} z} d z\right|^{2}
$$

where $R_{F}\left(q_{z}\right)$ is the Fresnel reflectivity of the ideal interface between the two media, $q_{z}$ is the scattering vector, and $\Delta \rho_{12}$ is the density contrast. In this formalism the $\rho(z)$ is the laterally averaged density profile. $R_{F}\left(q_{z}\right)$ can be written as $\left|\left(q_{z}-q_{z}^{\prime}\right) /\left(q_{z}+q_{z}^{\prime}\right)\right|^{2}$ with $q_{z}^{\prime 2}=q_{z}^{2}-q_{c}^{2}$. The critical momentum transfer is directly related to the density contrast by $q_{c}=4 \pi / \lambda \sin \left(\alpha_{c}\right) \simeq$ $4 \sqrt{\pi r_{0} \Delta \rho_{12}}$, with the classical electron radius $r_{0}$.

The lipid bilayer is separated from the solid support by a thin water layer [97], as illustrated in Figure 5.3.1. The electron density profile of a lipid bilayer on a Si substrate can be thus written as:

$$
\rho(z)=\left(\rho_{S i}-\rho_{\text {water }}\right) \cdot \operatorname{erf}\left(\frac{z+d_{0}}{\sqrt{2} \sigma}\right)+\rho_{0}(z)
$$

where $\left(\rho_{S i}-\rho_{\text {water }}\right)$ corresponds to the contrast of the interface between Si and the thin water layer, corrected by the error function with the rms substrate roughness $\sigma . \rho_{0}(z)$ represents the bilayer electron density.

\footnotetext{
${ }^{1}$ The semi-kinematic theory presented here combines the Fresnel-reflectivity, exact for an ideally flat interface between two media, with the kinematic master formula.
} 
(a)

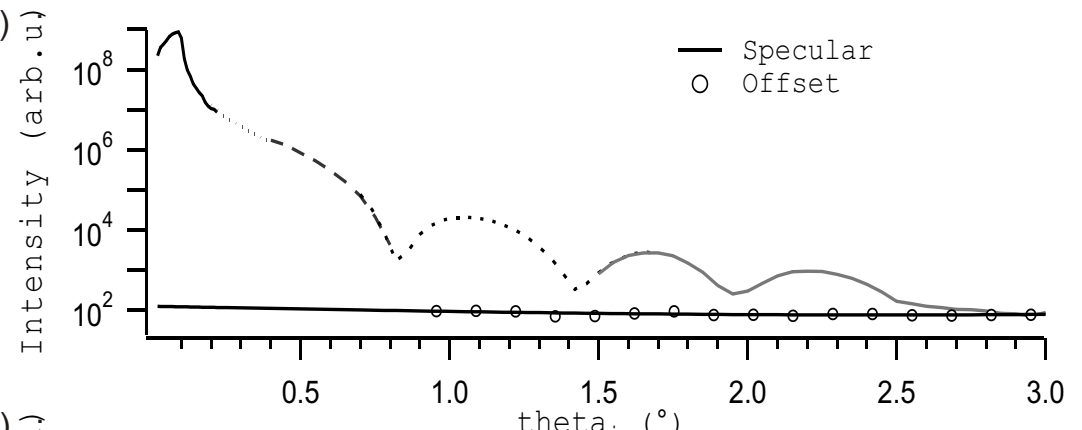

(b) $\bar{\jmath}$

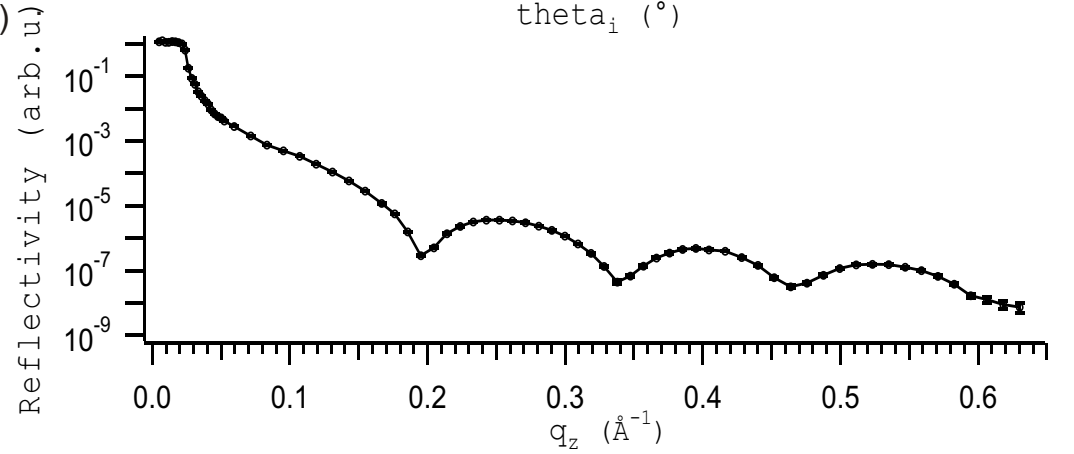

(c)

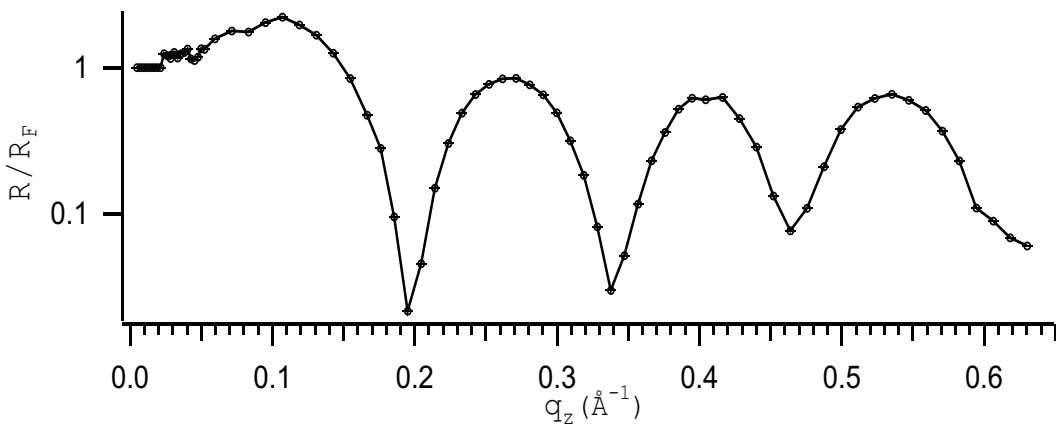

FIGURE 5.2.11. (a) X-ray reflectivity scan (before footprint correction) and offset-scan (non-specular background) of DOTAP/DOPC supported bilayer on silicon wafer, recorded at ID01. Different parts of the reflectivity scan (different symbols) were detected by using the different set of attenuators to prevent radiation damage. Obviously, for smaller $\theta_{i}$, more attenuators were used than for the higher $\theta_{i}$, until removing all attenuators for $q_{z} \simeq 1.6 \AA^{-1}$. Solid line corresponds to the polynomial fit to the measured background. (b) X-ray reflectivity scan after offset subtraction and illumination correction. The measured intensity is normalized to primary beam intensity. (c) Reflectivity scan divided by the Fresnel reflectivity of the silicon.

After inserting the electron density profile equation 5.3 .2 to equation (5.3.1) and taking the ensemble average, $\mathrm{X}$-ray reflectivity can be written as:

$$
\begin{array}{cc}
R\left(q_{z}\right)= & R_{F}\left(q_{z}\right) \mid \int \frac{1}{\sqrt{2 \pi \sigma^{2}}} e^{-\frac{1}{2}\left(\frac{z+d_{0}}{\sigma}\right)^{2}} e^{i q_{z} z} d z+ \\
+\left.\frac{1}{\Delta \rho_{12}} \int \frac{\partial \rho_{0}(z)}{\partial z} e^{i q_{z} z} d z\right|^{2}= \\
= & R_{F}\left(q_{z}\right)\left|e^{-i q_{z} d_{0}} e^{\frac{-q_{z}^{2} \sigma^{2}}{2}}+f\left(q_{z}\right)\right|^{2}= \\
= & R_{F}\left(q_{z}\right)\left[e^{-q_{z}^{2} \sigma^{2}}-2 i e^{-\frac{q_{z}^{2} \sigma^{2}}{2}} \sin \left(q_{z} d_{0}\right) f\left(q_{z}\right)+\left|f\left(q_{z}\right)\right|^{2}\right] .
\end{array}
$$


The first summand represents the reflectivity of the substrate. The second is a cross term and represents interference effects from the substrate with the bilayer. The third summand is the product of the form-factor $\left|f\left(q_{z}\right)\right|^{2}$, containing the structural information about the bilayer centered at $\mathrm{z}=0$ :

$$
f\left(q_{z}\right)=\int_{-\frac{d}{2}}^{\frac{d}{2}} \frac{1}{\Delta \rho_{12}} \frac{\partial \rho_{0}(z)}{\partial z} e^{i q_{z} z} d z .
$$

$\Delta \rho_{12}$ corresponds to the contrast of the water/Si interface, $d_{0}$ represents the thickness of a thin water layer with half membrane thickness (see Figure 5.3.1).

To describe the bilayer density profile $\rho_{0}(z)$ we chose the parametrization of the bilayer in terms of its first $N_{0}$ Fourier coefficients $f_{n}[\mathbf{8 9}]$,

$$
\rho_{0}(z)=\rho_{\text {water }}+\Delta_{\max } \Delta \rho_{12} \sum_{n=1}^{N_{0}} f_{n} v_{n} \cos \left(\frac{2 \pi n z}{d}\right) .
$$

The term $\left(\Delta_{\max } \Delta \rho_{12} \sum_{n=1}^{N_{0}} f_{n} v_{n} \cos \left(\frac{2 \pi n z}{d}\right)\right)$ can be understood as a deviation of the electron density of the bilayer from the electron density of surrounding water $\rho_{\text {water }}$ in terms of $f_{n} . v_{n}$ is the associated (complex) phase factor, which in our case can be shown to reduce to \pm 1 only, due to the mirror symmetry of the bilayer. $\Delta_{\max }$ is the amplitude of density deviation to the water density.

The integral of the form factor can be solved analytically after inserting equation (5.3.5) to equation 5.3.4, yielding

$$
f\left(q_{z}\right)=\sum_{n=1}^{N_{0}} f_{n} \Delta_{\max }\left[\frac{i 8 \pi^{2} n^{2} \sin \left(0.5 q_{z} d\right)}{q_{z}^{2} d^{2}-4 \pi^{2} n^{2}} \cos (n \pi)\right] .
$$

Finally, the expression (5.3.3) was used for the simulation (the computer program is listed in Appendix (C), where the parameters were optimized by a global search using a genetic optimization algorithm (Volker Türck, Optimize 5.02). The fitting procedure results in obtaining the electron density profile on an absolute scale. The fit parameters are reduced to the Fourier coefficients $f_{n}$, the associated phase factor $v_{n}$, the distance from the substrate to the middle of the bilayer $d_{0}$, the critical momentum transfer $q_{c}$, the amplitude of density deviation to the water density $\Delta_{\max }$ and the substrate roughness $\sigma$. The water electron density was fixed to $0.334 e^{-} / \AA^{3}$, and the silicon substrate electron density was fixed to $0.699 e^{-} / \AA^{3}$. The number $n$ of Fourier coefficients is adapted depending on the $q_{z}$ range, according to $n \simeq \frac{q_{\max }}{2 \pi D}$, where $D$ is the width of the film. Thus, the higher the range in momentum transfer $q_{\max }$, the higher the number of the Fourier coefficients (fit parameters) used. The fitting procedure often involves iterative changes in the number of Fourier coefficients.

The main purpose of the electron density profiles is to obtain structural information. The structural parameters can be derived from simple geometric relationships. The highest electrondensity peaks on both sides of the centrosymetric electron density profiles $\rho(z)$ of the bilayer coincide with the phospholipid head group $\rho_{h}$. The valuable quantity is the separation $d_{h h}$ of these maxima (head-to-head distance), which is a measure of the bilayer thickness. The two side minima correspond to the water layer and the central minimum is associated with the terminal

\footnotetext{
${ }^{2}$ http://sol.physik.tu-berlin.de/htm_trk/
} 


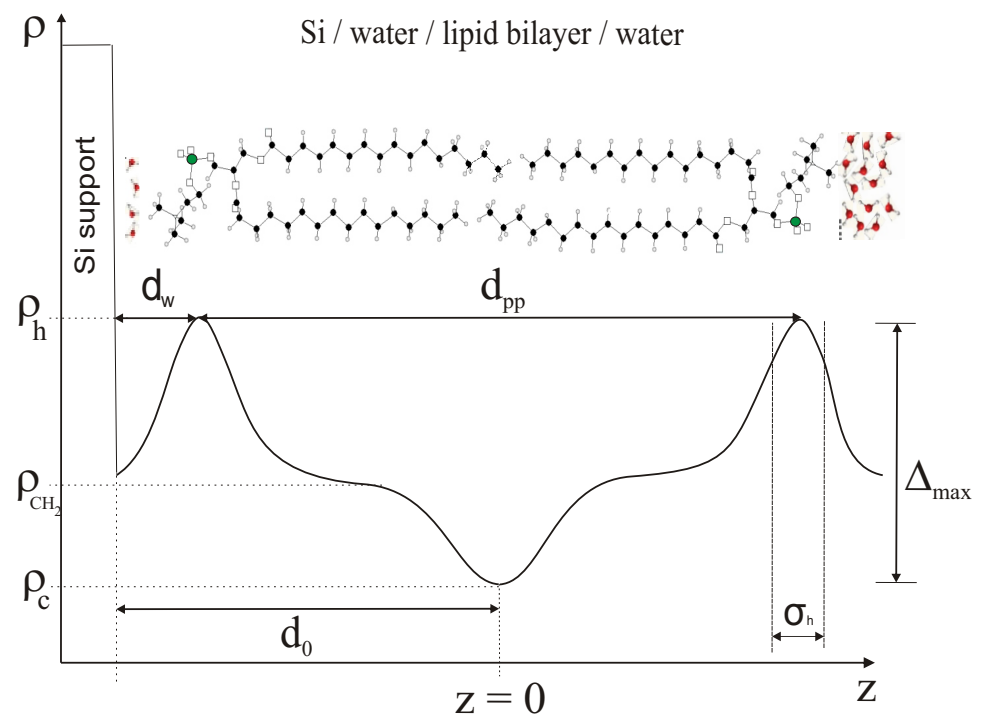

FIGURE 5.3.1. Model of the electron density profile $\rho(z)$ with the corresponding parameters used in the fitting procedure.

methyl groups of the hydrocarbon chains $\rho_{c}$. The thickness of the water layer between Si support and the bilayer, including the water molecules intercalated into the bilayer, can be determined as

$$
d_{\text {water }}=d_{0}-d_{h h} / 2
$$

The area per lipid $A$ is calculated from the relation given by Pabst et al. [ $\overline{\mathbf{9 8}}]$ :

$$
A=\frac{1}{\rho_{C H_{2}}\left(\frac{\bar{\rho}_{h}}{\bar{\rho}_{c}}-1\right)}\left(\frac{\frac{\bar{\rho}_{h}}{\bar{\rho}_{c}} n_{c}^{e}}{d_{c}}-\frac{n_{h}^{e}}{d_{h}}\right)
$$

where $n_{h}^{e}$ is the number the head-group electrons and $n_{c}^{e}$ number of hydrocarbon electrons. The electron densities of the head-group $\bar{\rho}_{h}$ and hydrocarbon tails $\bar{\rho}_{c}$ are defined relatively to the methylene electron density $\rho_{C H_{2}}\left(\bar{\rho}_{h}=\rho_{h}-\rho_{C H_{2}}, \bar{\rho}_{c}=\rho_{c}-\rho_{C H_{2}}\right)$. The methylene electron density, determined from wide-angle diffraction experiments, is $0.317 \pm 0.003 e^{-} / \AA^{3}$ [99]. The headgroup size $d_{h}$ is estimated from the full width at half maximum (FWHM) $\sigma_{h}$ of the Gaussian fitted to the head-group region, and the hydrocarbon chain length $d_{c}$ can be derived from

$$
d_{c}=\frac{d_{h h}-\sigma_{h}}{2} .
$$

The total number of water molecules per lipid molecule between the substrate and the bilayer, including the molecules intercalated into the bilayer, can be estimated from

$$
n_{w}=\frac{A d_{w}}{V_{w}},
$$

where $V_{w} \sim 30 \AA^{3}$ is the volume of one water molecule at $30^{\circ} \mathrm{C}$. We stress, however, that the values for $A, n_{w}, d_{c}$, and $d_{h}$ are derived from the density profile on the basis of a number of assumptions and definitions, as detailed in $[\overline{\mathbf{9 8}}]$. The primary structural results of the measurement is just the vertical density profile $\rho(z)$. 
TABLE 1.

\begin{tabular}{ccccc}
\hline Parameter & DOPC & $\begin{array}{c}\text { DOPC/DOPS } \\
(4: 1)\end{array}$ & $\begin{array}{c}\text { DOPC/DOTAP } \\
(9: 1)\end{array}$ & $\begin{array}{c}\text { DOPC/DOTAP } \\
(9: 1)^{(1)}\end{array}$ \\
\hline$\rho_{h}\left(e^{-} / \AA^{3}\right)$ & $0.45 \pm 0.13$ & $0.44 \pm 0.05$ & $0.43 \pm 0.03$ & $0.44 \pm 0.09$ \\
$\rho_{c}\left(e^{-} / \AA^{3}\right)$ & $0.23 \pm 0.07$ & $0.24 \pm 0.03$ & $0.24 \pm 0.02$ & $0.23 \pm 0.05$ \\
$d_{h h}(\AA)$ & $39.6 \pm 0.3$ & $39.4 \pm 0.2$ & $39.8 \pm 0.2$ & $40.0 \pm 1.1$ \\
$d_{w}(\AA)$ & $4.25 \pm 0.23$ & $3.9 \pm 0.2$ & $3.4 \pm 0.2$ & $3.9 \pm 1$ \\
$d_{h}(\AA)$ & $6.01 \pm 0.04$ & $6.30 \pm 0.15$ & $6.70 \pm 0.15$ & $5.60 \pm 0.10$ \\
$d_{c}(\AA)$ & $16.8 \pm 0.15$ & $16.6 \pm 0.1$ & $16.5 \pm 0.1$ & $17.2 \pm 0.55$ \\
$A\left(\AA^{2}\right)$ & $74.4 \pm 1.0$ & $71.1 \pm 1.3$ & $65.5 \pm 1.0$ & $72.9 \pm 1.35$ \\
$n_{w}$ & $10.6 \pm 0.2$ & $9.2 \pm 0.2$ & $7.4 \pm 0.1$ & $9.5 \pm 0.3$ \\
\hline
\end{tabular}

Derived values for electron density of the head-groups $\left(\rho_{h}\right)$ and of the terminal methyl groups of the hydrocarbon chains $\left(\rho_{c}\right)$, and derived structural parameters calculated by using Eqs. 55.3.7p- 5 5.3.10. ${ }^{(1)}$ are the values for D8 Advance measurements.

TABLE 2.

\begin{tabular}{cccc}
\hline Parameter & $\begin{array}{c}\text { DPPC/DPPS } \\
(4: 1)\end{array}$ & $\begin{array}{c}\text { DPPC/DPPS } \\
(1: 1)\end{array}$ & $\begin{array}{c}\text { DPPC/DPPS } \\
(1: 1)^{(1)}\end{array}$ \\
\hline$\rho_{h}\left(e^{-} / \AA^{3}\right)$ & $0.44 \pm 0.03$ & $0.44 \pm 0.01$ & $0.42 \pm 0.05$ \\
& & $\left(0.43^{*} \pm 0.01\right)$ & \\
$\rho_{c}\left(e^{-} / \AA^{3}\right)$ & $0.20 \pm 0.02$ & $0.19 \pm 0.02$ & $0.235 \pm 0.030$ \\
$d_{h h}(\AA)$ & $45.0 \pm 0.5$ & $49.8 \pm 0.1$ & $49.8 \pm 0.7$ \\
& & $\left(41.4^{*} \pm 0.08\right)$ & \\
$d_{w}(\AA)$ & $5.7 \pm 0.4$ & $4.9 \pm 0.2$ & $3.8 \pm 0.6$ \\
$d_{h}(\AA)$ & $13.7 \pm 1.6$ & $11.2 \pm 0.5$ & - \\
$d_{c}(\AA)$ & $15.6 \pm 0.8$ & $19.3 \pm 0.4$ & - \\
$A\left(\AA^{2}\right)$ & $43.3 \pm 2.6$ & $43.65 \pm 1.15$ & - \\
$n_{w}$ & $8.2 \pm 0.5$ & $5.5 \pm 0.7$ & - \\
\hline
\end{tabular}

Derived values for electron density of the head-groups $\left(\rho_{h}\right)$ and of the terminal methyl groups of the hydrocarbon chains $\left(\rho_{c}\right)$, and derived structural parameters calculated by using Eqs. 5.3.7 - 5.3.10. ${ }^{(1)}$ are the values for $\mathrm{D} 8$ Advance measurements. (*)corresponds to the values for the second maxima in the electron density profile of the DPPC/DPPS (1:1).

\subsection{Results and Discussion}

We measured specular X-ray reflectivity from the various lipid mixtures with low positive (DOPC/DOTAP (1:9)), neutral (DOPC) and negative charge (DOPC/DOPS (4:1), DPPC/DPPS (4:1), (1:1)). The lipid vesicles were deposited by vesicle fusion on a Si substrate as described in Chapter 3. Experiments were carried out at a constant temperature $23.8{ }^{\circ} \mathrm{C}$. We determined the best fit to the data for each sample by obtaining model intensity curves using the semi-kinematical approach described above. Reasonable starting parameter values were initially set for each fit. The resulting fit parameters are summarized in Tables 1 and 2 in Appendix A.

The intensity of specularly reflected X-rays for lipid mixtures was measured up to the momentum transfer $\sim 0.6 \AA$ for the bilayer in fluid phase and up to $\sim 0.7 \AA$ for the bilayer in gel phase, 
covering 8 orders of magnitude in intensity, before reaching the background level (see Fig. 5.4.1). For reflectivity measurements carried out at D8 Advance reflectometer, we were able to measure intensity of the reflected beam up to the momentum transfer $\sim 0.3 \AA$ for the bilayer in fluid phase and up to $\sim 0.35 \AA$ for the bilayer in gel phase (see Fig. 5.4.1).

An estimate of the spatial resolution $d_{\min }$ for the density profile obtained from a reflectivity measurement is given by the sampling theorem $d_{\min } \sim \frac{\pi}{q_{\max }}$, where $q_{\max }$ is the maximum momentum transfer of reflectivity measurement. This would imply $d_{\min } \sim 4.4 \AA$ (gel phase) and $\sim 5.2 \AA$ (fluid phase) for synchrotron measurements, and approximately two times higher values for inhouse reflectivity experiments. However, assuming the validity of a given model, for example the presence of the water layer between substrate and membrane, smaller distances influence the simulation also at smaller $q$ values. However, these results and the corresponding errors follow from the fitting procedure, and are quite model dependent.

5.4.1. Mixed bilayer in the fluid phase. Figure 5.4.1 shows the reflectivity curves for DOPC, DOPC/DOPS and DOPC/DOTAP lipid mixtures as a $\log _{10}$ (Reflectivity) vs. momentum transfer $q_{z}$. The solid curves represent the best fits to the reflectivity.

The main transition temperatures of DOPC, DOTAP and DOPS are below $0{ }^{\circ} \mathrm{C}$, therefore all compositions of these lipids, we used in our study, are expected to be in the fully hydrated fluid phase at $23.8^{\circ} \mathrm{C}$. The corresponding electron density profiles on the absolute scale $\left(e^{-} / \AA^{3}\right)$ are shown in Figure 5.4.2. Derived structural parameters are listed in Table1.

5.4.1.1. DOPC. The head-to-head distance of the DOPC bilayer was found to be $d_{h h}=$ $39.6 \AA$. This can be compared to the value of $37.1 \AA$ for multilamellar DOPC stacks on Si support at $30^{\circ} \mathrm{C}$, given by Liu et al. [100]. Leonenko et al. [101] measured by AFM the thickness of $40 \AA$ at $22{ }^{\circ} \mathrm{C}$ for supported DOPC bilayer deposited on mica.

The water layer thickness between Si substrate and the bilayer was found to be $d_{w}=4.3 \AA$. This is in a good agreement with the value $4 \AA$ for water layer between DOPC bilayer and quartz substrate, reported by Miller et al. [61]. The resulting electron density of the lipid heads is estimated to be $\rho_{h}=0.45 e^{-} / \AA^{3}$ and the electron density of lipid tails $\rho_{c}=0.23 e^{-} / \AA^{3}$. The values compare well to those obtained by diffuse X-ray scattering method [100] and X-ray reflectivity method [102]. The area per lipid has been calculated from Eq. 5.3.8. The number of head-group electrons is 164 , and the number of hydrocarbon chain electrons is 270 for DOPC. The obtained area per lipid was $A=74.4 \pm 1.0 \AA^{2}$. For comparison, Petrache et al. [103] found $A=72.5 \AA^{2}$ for DOPC in the fluid state at $30{ }^{\circ} \mathrm{C}$.

5.4.1.2. DOPC/DOPS(4:1). The value, we measured for the head-to-head distance of DOPC/DOPS (4:1) bilayer on silicon support, is $d_{h h}=39.4 \AA$, the electron density of lipid tails of $\rho_{c}=0.24 e^{-} / \AA$ and the electron density of lipid heads of $\rho_{h}=0.44 e^{-} / \AA^{3}$. We found the thickness of water layer between negatively charged substrate and negatively charged DOPC/DOPS(4:1) membrane to be lower $\left(d_{w}=3.9 \AA\right)$ than between the substrate and neutral DOPC membrane. The obtained area per lipid was $A=71.1 \pm 1.3 \AA^{2}$. For the calculation of the number of headgroup and hydrocarbon electrons of DOPC/DOPS lipid mixture, we took into the account the molar ratio of DOPC and DOPS in the mixture. The number of head-group electrons is 172, and 


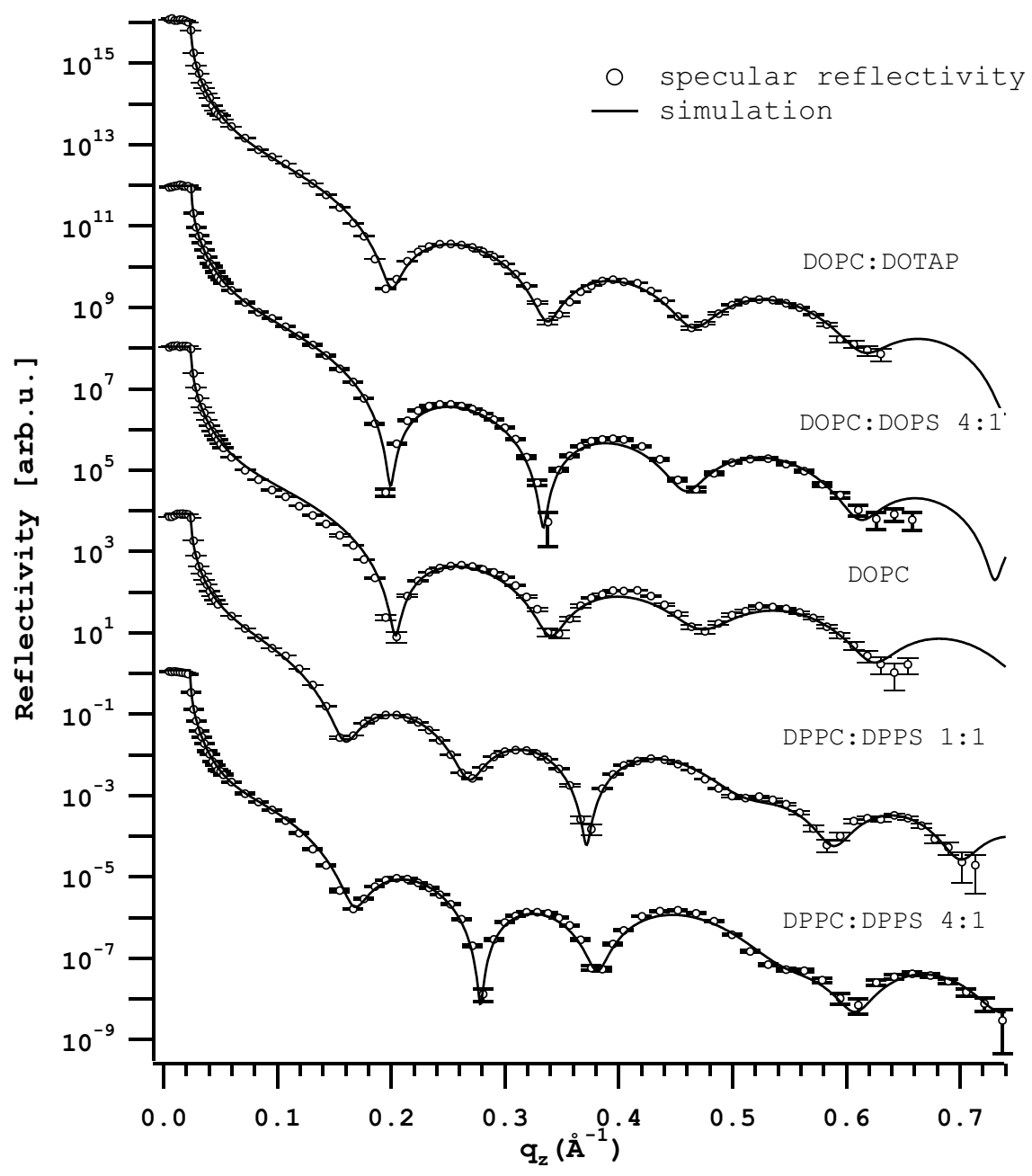

FIGURE 5.4.1. The best fit (solid lines) to the reflectivity curves of the different lipid mixtures in gel phase (DPPC/DPPS $(4: 1,1: 1)$ ) and in fluid phase (DOPC, DOPC/DOPS (4:1) and DOPC/DOTAP (9:1)) at $23.8^{\circ} \mathrm{C}$, recorded at the ID01 beamline.

number of hydrocarbon chain electrons is 270 for DOPS. The number of water molecules per lipid molecule between the bilayer and the silicon substrate was found to be approximately $n_{w} \sim 9.2$. The average area per lipid of DOPC/DOPS mixture was found to be smaller than the area of the DOPC and also $n_{w}$ is reduced by approximately 1.4 water molecules per lipid. Petrache at al. [103] studied structure of charged DOPS bilayer in the fluid state at $30^{\circ} \mathrm{C}$ and they reported the value $A=65.3 \AA^{2}$ for DOPS, what is remarkably smaller than their obtained value $A=72.2 \AA^{2}$ for DOPC.

To enhance bilayer formation from anionic lipids, we have added $2 \mathrm{mM} \mathrm{CaCl}_{2}$ to the buffer solution. It has been reported in the literature, that $\mathrm{Ca}^{2+}$ reduces the energy barrier that arises due to electrostatic repulsion between negatively charged substrate and lipids [49, 51]. It has been suggested that variations in $\mathrm{pH}$, salt concentration, and presence of divalent cations in the fluid 

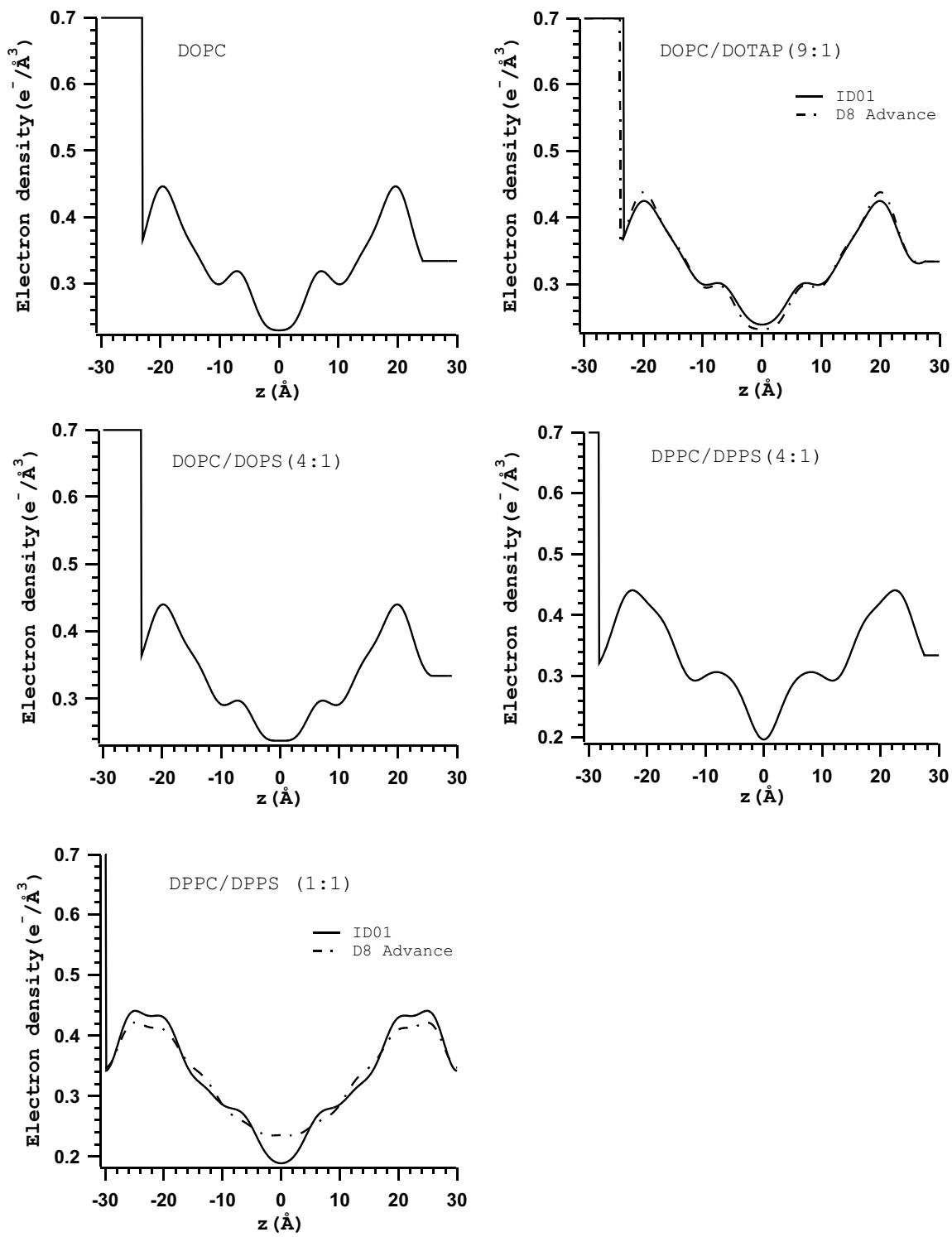

FIGURE 5.4.2. Electron density profiles corresponding to the simulations in Fig. 5.4.1 and 5.4.3.

phase may change the hydration properties of charged phospholipids [20]. We hypothesize that the effect of a thinner water layer can be due to lower area per lipid of DOPC/DOPS lipid mixture, in contrast to DOPC, or/and due to binding of the bivalent $\mathrm{Ca}^{2+}$ ions to negatively charged groups in DOPS.

5.4.1.3. DOPC/DOTAP(9:1). For a Si supported DOPC/DOTAP(9:1) membrane we obtained the head-group distance of $d_{h h}=39.8 \AA$, the electron density of the heads of $\rho_{h}=0.43 e^{-} / \AA^{3}$, and the electron density of the tails of $\rho_{c}=0.24 e^{-} / \AA^{3}$. This compares well to the literature values for DOPC/DOTAP(7:3), given by Yang et al. [104]. Molecular dynamics studies have demonstrated DOPC and DOTAP head groups to be located at a similar plane of the bilayer [105] and it was 
suggested that the quaternary amine of DOTAP and the phosphate group of DOPC may form a salt bridge. Here we find that the head-to-head distances $d_{h h}$ of DOPC/DOTAP mixture and DOPC are similar.

The obtained average area per lipid of DOPC/DOTAP was $A=65.5 \pm 1.0 \AA^{2}$. For a calculation of the number of head-group and hydrocarbon chain electrons of DOTAP/DOPC mixture, we took into the account the molar ratio of DOPC and DOTAP in the lipid mixture. The number of headgroup electrons is 126 , and the number of hydrocarbon chain electrons is 270 for DOTAP. The number of water molecules per lipid molecule was found to be $n_{w} \sim 7.4 \AA$. We found that the area of DOPC/DOTAP mixture is remarkably smaller than the area $74.4 \AA^{2}$ for DOPC, and $n_{w}$ is reduced approximately by 3 water molecules per lipid, comparing to DOPC supported bilayer. The thickness of head-group region $d_{h} \sim 6.7 \AA$ increased approximately by $0.7 \AA$, in contrast to DOPC.

Furthermore, we get $d_{w} \sim 3.4 \AA$ thin water layer between Si support and DOPC/DOTAP (9:1) membrane. This is $\sim 1 \AA$ less, respectively, than the value for DOPC membrane. This reduction of the water layer can be caused by lower area per lipid of DOTAP/DOPC mixture, in contrast to DOPC, or/and it could indicate electrostatic interactions between the low positively charged DOPC/DOTAP(9:1) membrane and the negatively charged silicon support.

5.4.2. Mixed bilayer in the gel phase and lateral demixing. Figure 5.4.1 shows the reflectivity curves for DPPC/DPPS $(4: 1,1: 1)$ lipid mixtures as a $\log _{10}$ (Reflectivity) vs. $q_{z}$. The solid curves represent the best fits to the reflectivity. The phase transitions of DPPC and DPPS from the order gel phase to the disordered liquid-crystalline phase are reported in the literature to occur at $41.4{ }^{\circ} \mathrm{C}$ and $54{ }^{\circ} \mathrm{C}[20]$. Therefore, we expect to have DPPC/DPPS mixtures at experimental temperature $23.8^{\circ} \mathrm{C}$ in gel phase. The corresponding electron density profiles are shown in Figure 5.4.2(d,e). The structural parameters are listed in Tab. 2

5.4.2.1. DPPC/DPPS(4:1). From the analysis of the simulation for reflectivity profile of DPPC/DPPS (4:1) bilayer, we obtained the electron density of lipid heads of $\rho_{h}=0.44 e^{-} / \AA^{3}$ and the electron density of lipid tails of $\rho_{c}=0.2 e^{-} / \AA^{3}$. The thickness of the bilayer was found to be $d_{h h} \sim 45 \AA$. The obtained average area per lipid was $A=43.3 \pm 2.6 \AA^{2}$. For the calculation of the number of head-group and hydrocarbon electrons for DPPC/DPPS lipid mixture, we took into the account the molar ratio of DPPC and DPPS in the mixture. The number of head-group electrons is 164 for DPPC and 172 for DPPS, and the number of hydrocarbon chain electrons is 242 for DPPC and DPPS. For comparison, Wiener et al. [99] measured the values: $\rho_{h}=0.47 e^{-} / \AA^{3}$, $\rho_{c}=0.21 e^{-} / \AA^{3}, d_{h h}=45 \AA$ and $A=45.9 \pm 2.0 \AA^{2}$ for gel phase DPPC bilayer at $\mathrm{T}=20{ }^{\circ} \mathrm{C}$ by $\mathrm{X}$-ray scattering methods.

The water layer between the silicon and DPPC/DPPS (4:1) was found to be $\sim 5.7 \AA$.

5.4.2.2. DPPC/DPPS(1:1). In the case of DPPC/DPPS (1:1) bilayer, we observe a splitting of the head-group region into two parts, with the corresponding electron densities of lipid heads $\rho_{h}=0.44 e^{-} / \AA^{3}$ and $\rho_{h}^{*}=0.43 e^{-} / \AA^{3}$, and with the head-group separations of $d_{h h}=49.8 \AA$ and $d_{h h}^{*}=41.4 \AA$, respectively. We hypothesize that this separation in the head-group region is likely 
to be caused by calcium-induced lipid segregation in the film. However, more experiments are needed to verify lateral segregation.

Onishi and Ito [106] observed calcium-induced clustering of PC molecules in PS/PC membrane. PS molecules, on the other hand, form solid aggregates bridged by intermolecular calcium chelation, causing motional freezing of lipids. They concluded, that the motional freezing results from closer packing of lipid molecules in the bilayer structure. The closer packing of lipid molecules could explain our observation of the larger distance between two outer head-group maxima in the electron density profile of the DPPC/DPPS(1:1) bilayer, $d_{h h}=49.8 \AA$, comparing with the head-group distance of DPPC/DPPS( $4: 1)$ bilayer of $d_{h h}=45 \AA$. We hypothesize, that the structure of $\rho(z)$ reflects the following components (after lateral average in the plane of the bilayer): the water layer, an outer head-group region of closely packed DPPS molecules, DPPS tails with DPPC head-groups and mixed tails of DPPC and DPPS molecules.

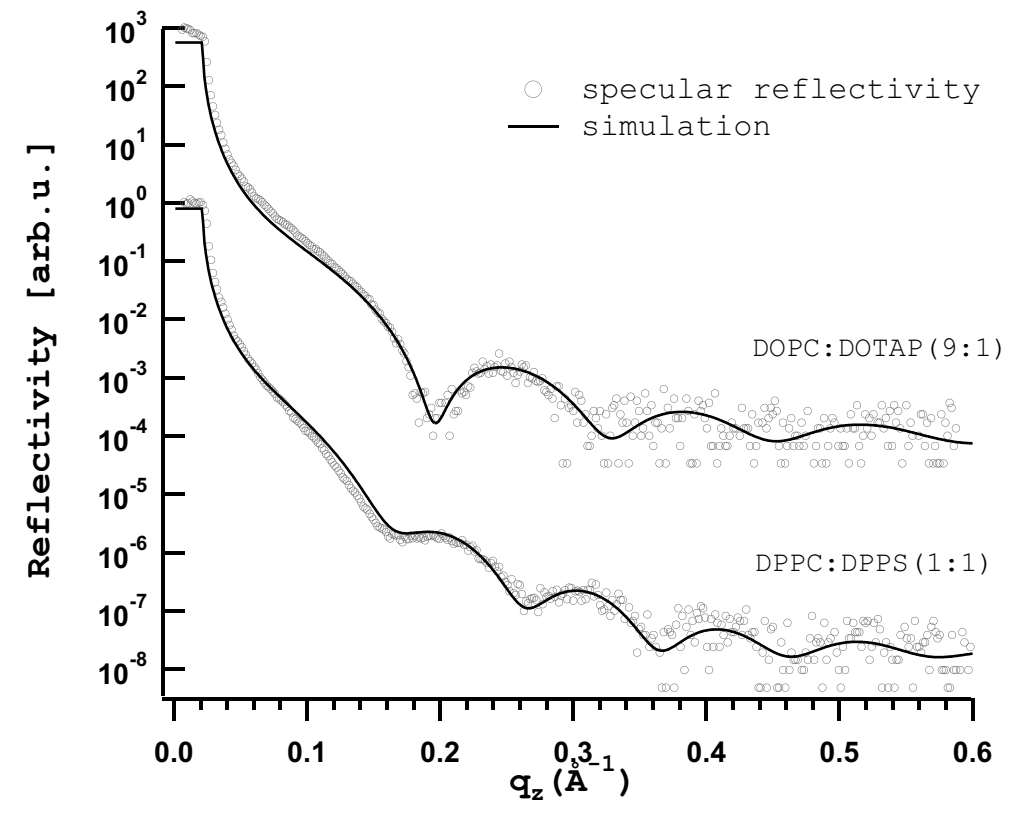

FIGURE 5.4.3. Experimental reflectivities recorded at D8 Advance reflectometer. Solid lines represent the best fit curves.

Onishi and Ito [106] furthermore noted, that the motive force for the phase separation is formation of Ca-PS aggregates in the bilayer. Jacobson at al. [107] found out, that $\mathrm{Ca}^{2+}$ ions added to mixed bilayers can induce lateral phase separation isothermally, with the neutral PC molecules segregating from acidic PS. They observed, that calcium ions do not induce phase separation below a critical concentration and, then only with mixtures containing more than $50 \%$ PS. This could explain, why we did not observe the segregation of lipids in DPPC/DPPS(4:1) supported bilayer. Another explanation would be a possibly insufficient resolution to detect the separation in DPPC/DPPS(4:1) mixture. Note that Ross et al. [108] have imaged calcium-induced domains in DPPC/DPPS(4:1) by AFM in Langmuir-Blodgett layers in the presence of $0.1 \mathrm{mM} \mathrm{CaCl}_{2}$. They 


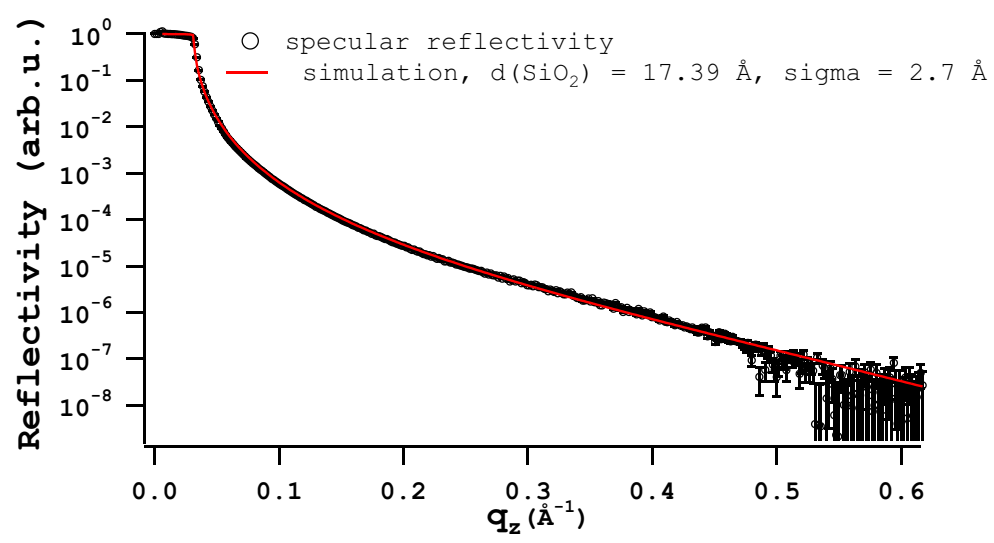

FIGURE 5.4.4. Reflectivity curve of the Si/native silicon oxide layer. The best fit (red solid line) is plotted.

observed, that even negligibly small traces of calcium ions present in the buffer solution lead to their accumulation at negatively charged lipid domains.

The thickness of the water layer between DPPC/DPPS (1:1) bilayer and the silicon support $\left(d_{w} \sim 4.9 \AA\right)$ was reduced, compared to DPPC/DPPS(4:1) bilayer. The thinning of the water layer could be explained by increased attractive electrostatic forces between negatively charged substrate and the DPPC/DPPS(1:1) bilayer, caused by the higher concentration of $\mathrm{Ca}^{2+}$ ions binding to the lipid head-groups. The average area per lipid obtained by in-house experiment was $A=43.65 \pm 1.15 \AA^{2}$ for DPPC/DPPS(1:1) lipid mixture.

5.4.3. Reflectivity measurements of the dry silicon substrate. Silicon wafers, exposed to air after their fabrication process, possess a surface layer of naturally grown silicon oxide, or silica. We measured by x-ray reflectivity the thickness of silicon oxide layer and the roughness of the surface. The substrate, a polished silicon (100) wafer, was cleaned by a standard procedure (Chapter 3). The empty wafer was characterized by in house x-ray reflectivity (measured up to $q_{z}=0.62 \AA^{-1}$ ) measurement . Reflectivity data of the dry substrate, after subtracting the offset and normalizing to the primary beam intensity are plotted (Fig. 5.4.4).

The Parratt32 software [109] was used to fit model parameters to the measured reflectivity data. The fit results indicate that the surface is covered with a native oxide layer of $d_{\mathrm{SiO}_{2}} \sim 17.39 \AA$ thickness. In the literature, the thickness of this silicon oxide layer is between 6 and $20 \AA$, its nominal value depending on oxidation conditions and the measurement techniques used [75]. A rms-roughness of the air-oxide interface was found to be $\sigma_{\mathrm{SiO}_{2}} \sim 2.7 \AA$. This is in agreement with our AFM measurements (see Fig. 3.2.3 (b)) of the roughness. The density of silicon-based oxides can vary from 2.17 to $2.66 \mathrm{~g} / \mathrm{cm}^{3}[\mathbf{1 1 0}]$. In our simulation the density of the silicon oxide was kept to be $\rho_{\mathrm{SiO}_{2}}=2.2 \mathrm{~g} / \mathrm{cm}^{3}$, which is the standard for amorphous silica. The electron densities of silicon and silicon dioxide are very similar $\left(\rho_{\mathrm{SiO}_{2}} / \rho_{\mathrm{Si}}=0.95\right)$ [111]. In this work, a separate silicon oxide layer was not taken into account to simulate the reflectivity curves of the different lipid mixtures. 


\subsection{Summary and Conclusions}

Let us briefly compare structural analysis using single supported bilayers, multilamellar thin films, and multilamellar liposome suspensions (probed by bulk SAXS). In all three cases, a wide $q_{z}$-range can be measured and analyzed by full $q_{z}$-fitting $[\mathbf{8 9},[112,[98]$. However, multilamellar bilayer suspension undergoes much stronger thermal fluctuations, in particular in bulk phases, while the presence of the flat substrate quenches the thermal fluctuations on long lateral length scales leading to an enhanced resolution in $\rho(z)$. This is an advantage in using solid supported membranes, either single bilayers or stacks. Furthermore the membranes can be investigated along both symmetry axis perpendicular or lateral independently [113, 114]. While thick stacks provide the large scattering volume needed for lateral structure analysis, specular reflectivity is not carried out easily on aligned stacks as on single bilayers. Stacks require a precise modeling of the structure factor including effects of thermal inter-bilayer positional fluctuations and coverage [112], while the analysis of single bilayers is more rapid and it is significantly easier to achieve high quality fits. Single bilayer reflectivity also allows for the highest resolution obtainable in the fluid state and at full hydration. Furthermore, we have shown that such analysis does not rely only on synchrotron radiation, but can also be carried out at sealed tube instruments with optimized equipment. We have shown that the resolution is high enough to detect a splitting in the head-group maximum, which we attribute to lateral demixing of the two-component system. Diffuse X-ray scattering can be used in future to support this conclusion and to determine the lateral size of the domains. On the other hand, the presence of the substrate may lead to distortions and small structural changes of the bilayer. The water layer $d_{w}$ between silicon and the head-group of the lower leaflet can be determined with high precision, and is quite small $d_{w} \leq 6 \AA$ for all samples, indicating that the bilayers are rather close to the solid surface. The system can thus be regarded as a 'pinned bilayer', in contrast to the free floating membranes, which can be achieved by preparation of a bilayer on top of a monolayer [115, 116]. 



\title{
CHAPTER 6
}

\section{X-ray spectro-microscopy study of solid supported two-component lipid membranes mixed with charged colloids}

\begin{abstract}
6.1. Introduction
A quantitative understanding of the interactions between nanoparticles and biological interfaces, in particular the cell membrane, is a prerequisite for the design of drug delivery systems based on synthetic nanoscale carrier systems, as well as for imaging agents such as fluorescent quantum dot markers. Functionally relevant issues are related to the binding affinity, the range of interaction, possible toxic effects due to membrane disruption or lysis [14, 15, 16], as well as the lateral reorganization of a multi-component membrane in response to nanoparticle binding [17]. The dominant interaction forces between nanoparticles and membranes are electrostatic in nature, since charged lipids are common and versatile constituents of biological membranes [23]. Charges in such lipids differ in magnitude and location within the interface and can locally demix in response to charged nanoparticles . If binding of nanoparticles to a multi-component membrane results in a re-distribution of charged and uncharged lipids, this altered membrane state will in return affect the bilayer-nanoparticle interaction, so that strong non-linear effects can be expected. Colloids are able induce bending deformations in charged membranes, giving rise to various phenomena such as partially, or fully wrapping of the membrane around the colloid [17, 69]. Electrostatic adsorption of colloids on a two-component membrane with ideal mixing properties can lead to compositional inhomogenities at the colloids adsorption sites [17]. From a fundamental point of view, the interaction between two classical model systems, the lipid bilayer on one side, and colloidal nanoparticles on the other, can serve as well-controlled testing ground for concepts in colloid science and soft condensed matter physics.

These important topics can be addressed using a solid-supported phospholipid membrane as a biomimetic model for nanoparticle-phospholipid interactions, since geometry, composition and environmental parameters can be better controlled in a biomimetic system than in a cell [87]. Moreover, planar bilayer systems are amenable to quantitative high-resolution analytical techniques, which can not (or not yet) be applied to real biological membranes. The biophysical techniques carried out on planar bilayers range from infrared spectroscopy, plasmon resonance, X-ray and neutron reflectivity, to fluorescence microscopy. Many of these involved charged species. The absorption of highly charged biomolecules such as DNA with the cationic and mixed bilayers on solid surfaces has been studied by fluorescence microscopy $[\mathbf{8 8}]$. The structure of two-component lipid mixtures, with varied surface charges, both in the fluid and gel phase, has been probed by using X-ray reflectivity [60].
\end{abstract}


A particular technique which may be useful to such systems, but which has to date not been fully exploited, not even for studies of pure lipid membranes, is synchrotron-based soft X-ray microscopy and spectro-microscopy. This techniques can provide the required combination of chemical sensitivity and high spatial resolution, in particular to probe the lateral organization of a multi-component membrane. In near-edge X-ray absorption (NEXAFS) spectro-microscopy, inner-shell electronic excitations are used as a chemically sensitive image-contrast mechanism [18]. Hitchcock et al. [117] have shown that while scanning transmission X-ray microscopy is essentially a "bulk" technique, protein monolayer detection is possible, although close to the current limit of sensitivity.

In this work we have used scanning transmission X-ray microscopy to image positively charged polystyrene latex microspheres which bind onto a supported two-component lipid membrane, composed of charged and neutral lipid species. We used protocols for the deposition of solid supported lipid bilayers by vesicle fusion [13], which have been adapted for deposition of charged bilayers [49]. Carbon $K$-edge NEXAFS spectroscopy is particularly favorable for studies of phospholipids since they have a strong $\pi^{*}(C=O)$ resonance at $\sim 288.5 \mathrm{eV}[\mathbf{1 1 8}]$. We have tested the ability of scanning transmission X-ray spectro-microscopy to examine samples under physiologically relevant conditions in the presence of excess water. In the energy range of the so called "water window" between oxygen and the carbon $K$ absorption edges ( $283 \mathrm{eV}$ and $543 \mathrm{eV})$, specimens in water show a natural absorption contrast, which permits imaging of unstained specimens in a hydrated state. The samples are then imaged both below and above the carbon $K$ absorption edge. The amount of the carbon can be determined quantitatively from the difference in the $\mathrm{X}$-ray absorption. The thickness of the lipid layer and the microspheres can then be determined. Spectro-microscopy data can be extended to a sequence of images over a series of spectroscopically interesting energies, providing spatially and spectrally resolved data of the sample simultaneously [119]. Such image stacks have been collected on dried colloids as well as wet lipid membrane/polystyrene microspheres.

A further, more technical motivation for the present study was to demonstrate the feasibility of lipid thin films carbon $K$-edge NEXAFS experiments with X-ray radiation generated from compact sources based on a laser-produced plasma. NEXAFS experiments have almost exclusively required synchrotron radiation before. Peth et al. [120] have recently presented NEXAFS results that were obtained by using a laboratory scale laser-driven plasma source. In this work, we show the NEXAFS spectra of selected lipids, which differ in the headgroup structure or/and in containing the $\mathrm{C}=\mathrm{C}$ double bond in hydrocarbon tails. $\pi^{*}(C=C), \pi^{*}(C-C), \sigma^{*}(C=C), \sigma^{*}(C-C)$ and Rydberg resonances, and $\mathrm{C} 1 s \rightarrow \sigma^{*}(C-N)$ transitions were resolved.

\subsection{Near-edge $X$-ray absorption fine structure}

The intensity of X-rays transmitted through a sample decreases due to their absorption. Depending on the energy, the absorbed X-rays can excite core electrons from different levels in an atom or a molecule. With the increase of photon energy, the X-ray absorption cross section that describes the probability of absorption decreases. However, when the photon energy corresponds to the binding energy of a core electron, the X-ray absorption cross section increases abruptly, and 
then decreases monotonically above the core edge. Thus an edge-like structure is observed in the absorption spectrum. The simplest method for X-ray absorption spectroscopy (XAS) is a transmission XAS experiment. The transmitted X-ray intensity $(I)$ through a material depends on the thickness, density, atomic number of the material and the energy of individual photons. According to the Beer-Lambert's law

$$
I(E)=I_{0}(E) \exp (-\mu(E) d)
$$

where $I_{0}$ is the incident intensity and $d$ is the material thickness. $\mu$ is the linear absorption coefficient in units of 1/length, which can be expressed for a single element as:

$$
\mu=\frac{4 \pi}{\beta}=2\left(N_{A} / M\right) r_{e} \rho \lambda f_{2}(\lambda)
$$

where $\beta$ is the imaginary part of the index of refraction $n$, derived in chapter $4 . N_{A}$ is Avogadro's number, $M$ is a molar mass, $r_{e}=2.818 \times 10^{-15} \mathrm{~m}$ is the classical radius of the electron, $\rho$ the element's density, $\lambda$ the X-ray wavelength and $f_{2}(\lambda)$ is the imaginary part of the complex scattering factor, tabulated for all relevant elements in the soft X-ray energy range [84]. Compounds and molecules can be modeled, away from the resonances, by calculating $f_{2} / M$ as a weighted sum over all elements. The intensity absorption is also commonly expressed by the mass attenuation coefficient $\mu_{m}=\mu / \rho$. In XAS, the dependence of the absorption coefficient $\mu$ on the wavelength of the incoming X-ray beam, so-called optical density $\left(O D=\mu d=-\ln \left(I / I_{0}\right)\right)$, is measured.

The absorption coefficient $\mu$ is related to the X-ray absorption cross-section per atom, $\sigma_{a}$, through

$$
\mu=\left(\frac{\rho_{m} N_{A}}{A}\right) \sigma_{a}
$$

where $N_{A}, \rho_{m}$ and $A$ are Avogadro's number, the mass density, and atomic mass number, respectively $[\mathbf{8 1}]$.

6.2.1. X-ray absorption cross-section. The absorption cross section of an atom or a molecule is defined as the number of electrons excited per unit time divided by the number of incident photons per unit time per unit area [18]. In a quantum mechanical treatment the scattering process is described by time dependent perturbation theory. The cross section can be calculated from Fermi's Golden Rule for the transition probability per unit time $P_{i f}$ from a state $|i\rangle$ into an unoccupied state $|f\rangle$ driven by a harmonic time-dependent perturbation $V(t)=\bar{V} e^{-i \omega t}$

$$
P_{i f}=\frac{2 \pi}{\hbar}|\langle f|\bar{V}| i\rangle|^{2} \rho_{f}(E)
$$

with $\rho_{f}(E)$ being the energy density of final states. In the case of $K$-shell excitation $P_{i f}$ is the number of electrons excited per unit time from the $1 s$ shell to a final state $|f\rangle$ which, in principle, can be a bound or continuum state and $\hbar=h / 2 \pi, h$ is the Planck constant. The inner shell excitation is produced by an electromagnetic wave of energy $\hbar \omega$, which is described as a plane wave in the Coulomb gauge $(\Phi=0, \operatorname{div} A=0)$ : 


$$
\vec{A}=\vec{e} \frac{A_{0}}{2}\left(e^{i(\vec{k} \vec{x}-\omega t)}+e^{-i(\vec{k} \vec{x}-\omega t)}\right)
$$

$\vec{A}$ is the vector potential in the form of a plane electromagnetic wave of wave vector $\vec{k}$, frequency $\omega$, and unit vector $\vec{e}$. The absorption cross section is then obtained as

$$
\sigma_{a}(E)=\frac{P_{i f}}{F_{p h}}
$$

where $F_{p h}=\frac{E^{2} c}{8 \pi \hbar \omega}$ is the photon flux associated with this plane wave [121].

The perturbative terms describing the interaction of spinless particles of charge $-e$ and mass $m$ with an electromagnetic field is given by [122].

$$
V(t)=\frac{e}{m c} \vec{A} \cdot \vec{p}
$$

where $\vec{p}=\sum \vec{p}_{i}$ is the sum of linear momentum operators of the electrons. Substituting Eq. 6.2.7 and Eq. 6.2.5 into Eq. 6.2.4, and realizing that only the time dependent term $e^{-i \omega t}$ in Eq. 6.2.5 causes transitions that absorb energy, we obtaine for the transition probability per unit time

$$
P_{i f}=\frac{\pi e^{2}}{2 \hbar m^{2} c^{2}} A_{0}^{2}\left|\left\langle f\left|e^{i \vec{k} \vec{x}} \vec{e} \cdot \vec{p}\right| i\right\rangle\right|^{2} \rho_{f}(E) .
$$

Following Stöhr [ [18], in the dipole approximation ${ }^{1}$ the $\mathrm{X}$-ray absorption cross section is given by

$$
\sigma_{a}(E)=\frac{4 \pi^{2} \hbar^{2}}{m^{2}} \frac{e^{2}}{\hbar c} \frac{1}{\hbar \omega}|\langle f|\vec{e} \cdot \vec{p}| i\rangle|^{2} \rho_{f}(E) .
$$

$\sigma_{a}(E)$ is usually expressed in barn $\left(1 \mathrm{~cm}^{2}=10^{24}\right.$ barn $)$.

6.2.2. Transition energies. The absorption of an X-ray photon can excite electrons of $1 s$ ( $K$ edge) or $2 s, 2 p$ ( $L$ edge) states to empty localized orbitals or, for high-energy X-ray photons, to the continuum. An important quantity in the discussion of $K$-shell excitation spectra is the $1 s$ ionization potential, defined as the minimum energy necessary to excite a $1 s$ electron to the continuum of states above the vacuum level. The ionization potential $I P(i)$ or binding energy $E_{b}(i)$, associated with a particular electron $i$ in an atom or a molecule is

$$
I P(i)=E_{b}(i)=\hbar \omega-E_{k i n}
$$

In $K$-shell X-ray absorption, the $1 s$ IP is the threshold energy (usually characterized by an absorption step) for transitions to continuum states. Near the K-shell absorption threshold, a series of fine structures are superimposed on the absorption edge. In organic molecules, these fine structures are dominated by resonances arising from $1 s \rightarrow \pi^{*}$ or $1 s \rightarrow \sigma^{*}$ transitions, depending on

\footnotetext{
${ }^{1}$ The dipole approximation assumes $k \cdot x \ll 1$ or $|x| \ll \lambda / 2 \pi$.
} 

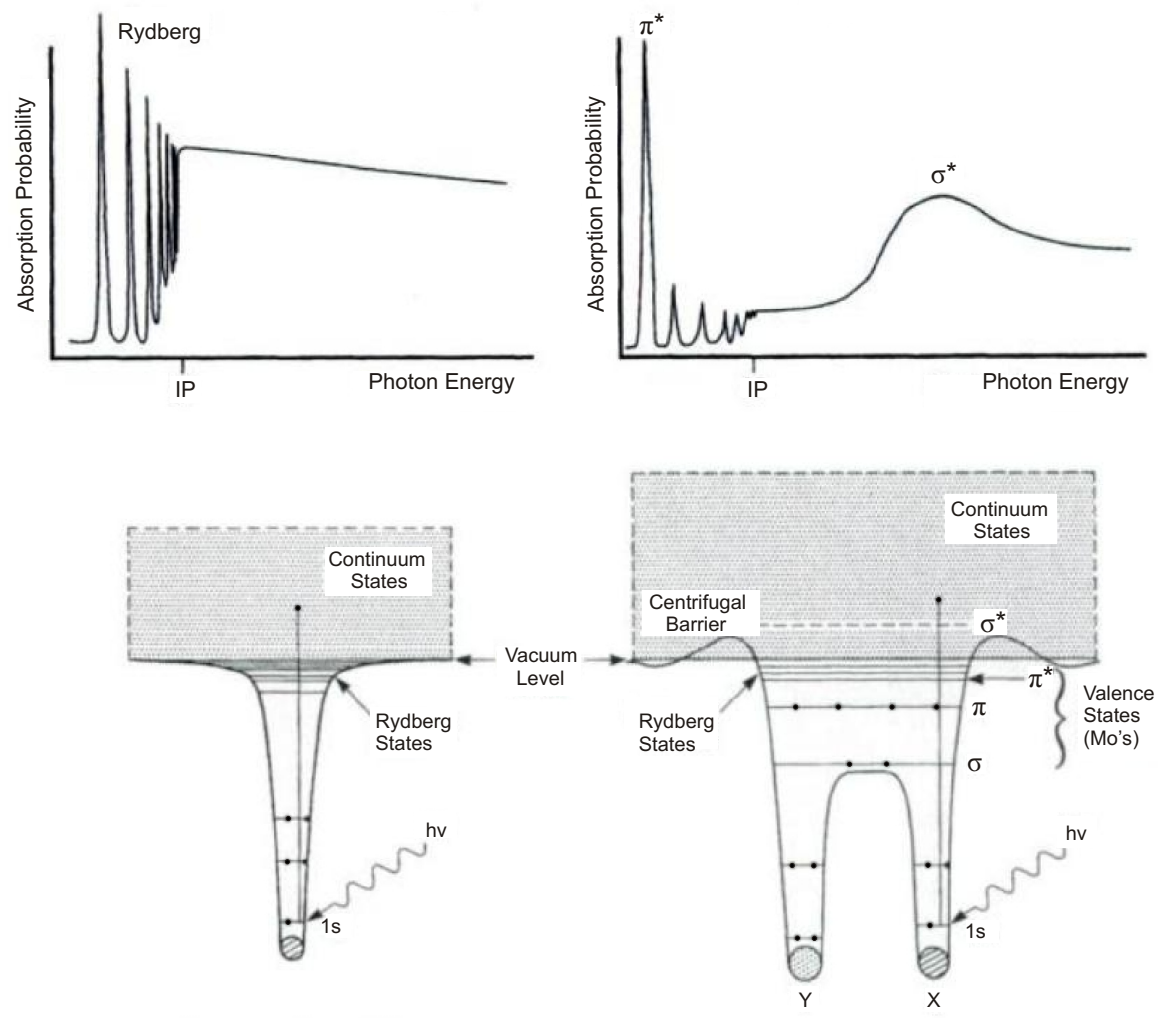

FIGURE 6.2.1. Schematic potentials (bottom) and $K$-shell spectra (top) of single atoms and diatomic molecules taken from [18].

the covalent bonding in the molecule, as well as to Rydberg orbitals. The absorption spectrum of an element near the X-ray absorption edge, so-called NEXAFS, is usually dominated by two types of resonances, with the ionization potential as a boundary. Resonances below the IP correspond to the excitation of a core electron to a bound orbital. These orbitals are usually of $\pi^{*}$ or Rydberg character and sometimes of $\sigma^{*}$ character for saturated species. Resonances above the IP usually correspond to the excitation of a core electron to an unbound orbital of $\sigma^{*}$ character, as well as double excitation. These resonances are usually broad. In Fig. 6.2.1, schematic of characteristic resonances in $K$-shell spectra and a step-like increase at the IP, are shown.

Generally, the X-ray absorption spectrum can be divided into two parts which differ in their physical origin. The X-ray absorption near-edge structure (XANES), also called Near Edge X-ray Absorption Fine Structure (NEXAFS), comprises the regime from $-10 \mathrm{eV}$ below, to about $+50 \mathrm{eV}$ above the edge energy. This regime is characterized by rather sharp variations of the absorption. It is subdivided further into the pre-edge region and the region directly above the edge. In the part from ca. $+50 \mathrm{eV}$ to ca. $+1000 \mathrm{eV}$ above the edge energy, weak oscillations in the absorption fine structure occur. These are designated as extended X-ray absorption fine structure (EXAFS). EXAFS spectroscopy is used mostly in the study of inorganic species for determining the numbers, types, and distances of the backscattering atoms surrounding the absorbing atoms [123]. However, 
core edges in low $\mathrm{Z}$ atoms are too low in energy to permit EXAFS analysis. For organic molecular species, the NEXAFS part of the spectrum is the most useful.

\subsection{NEXAFS contrast soft X-ray spectromicroscopy}

In spectromicroscopy the chemical mapping is obtained by utilizing the large absorption coefficient changes that occur at the absorption edges of elements. Chemical sensitivity is then obtained recording images with a scanning or a fullfield microscope at a number of different energies appropriately selected in order to differentiate the chemical components of the system. Each image pixel provides a certain absorption spectrum which is the weighted sum of the absorption spectra of the different components. The entire image sequence can then provide, after careful analysis, the chemical composition at each sample location, or conversely the spatial distribution of each element, with the accuracy of the optical system in use [124] which can be as good as few tenths of nanometers.

Fig. 6.3.1 shows the schematic setup of the STXM. The monochromatic beam is focused by the zone plate and forms a microprobe which is used to scan the sample by moving the zone plate. The transmitted beam is measured with a fast detector. A central stop on the zone plate and a so-called order sorting aperture (OSA) select the light of the first diffraction order to illuminate the sample and block the light from the zeroth and from higher orders.

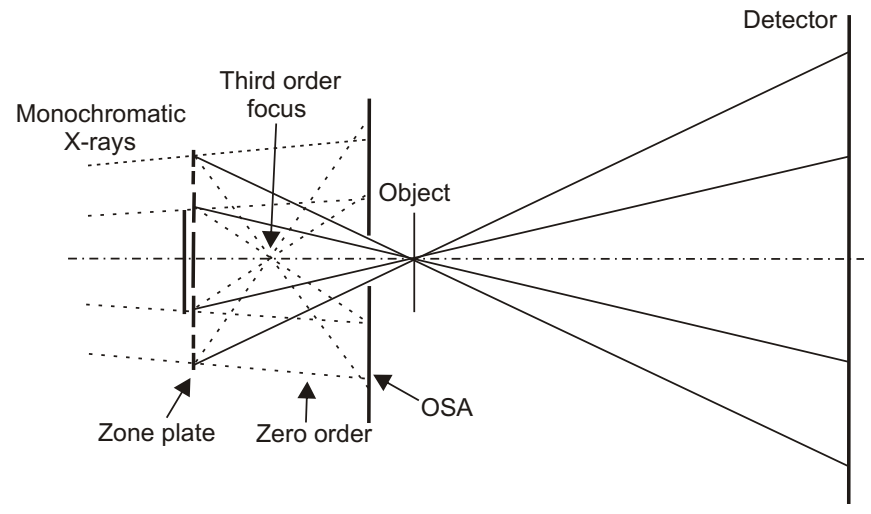

FIGURE 6.3.1. Schematic diagram of the STXM operating in the first diffraction order of the zone plate.

Zone plates were invented about 120 years ago by Rayleigh, and independently by Soret [125]. These are circular diffraction gratings with radially increasing line density. Following a proposal by Schmahl and Rudolph [126], the first successful scheme for fabricating zone plates for X-ray microscopy using holography was developed by Niemann et al. [127].

These diffractive focusing elements are made up of concentric circular zones, with Fresnel half-period zone radii given by the approximate relationship [125]

$$
r_{n}^{2}=m n \lambda f+m^{2} n^{2} \lambda^{2} / 4
$$


where $n$ is the zone number, $f$ the focal length, $\lambda$ is the wavelength, $m$ denotes the diffractive order ( $m=1$ is the order usually used for imaging), and the $m^{2} n^{2} \lambda^{2} / 4$ term is for correction of spherical aberration assuming a source or object at an infinite distance from the zone plate. They work like thin lenses, exhibiting a diffraction limit to their transverse resolution $\delta_{t}$ of

$$
\delta t=0.61 \frac{\lambda}{m \mathrm{NA}}
$$

as calculated by Rayleigh for a lens with numerical aperture NA. Two other especially useful relationships can be obtained from these expressions if the $m^{2} n^{2} \lambda^{2} / 4$ spherical aberration correction term is neglected [128]. One is the relationship between the width of the smallest zone $\delta_{r_{N}}$ of a zone plate with $N$ half-period zones and its Rayleigh resolution of

$$
\delta t=1.22 \delta_{r_{N}} / m
$$

Another is the relationship between the diameter $d=2 r_{N}$, outer zone width, focal length, diffractive order and illumination wavelength of

$$
d \delta r_{N}=m f \lambda
$$

To deliver optimum performance [129], a zone plate should be illuminated with radiation with a monochromaticity equal to the total number of half-period zones multiplied by the diffractive order, or

$$
\frac{\lambda}{\triangle \lambda} \gtrsim m N
$$

Spectral properties of sample constituents also form the physical basis for a contrast mechanism in a direct X-ray image formation. Combining this X-ray spectral fingerprint with direct high resolution X-ray imaging is termed NEXAFS spectro-microscopy. In biological imaging of organic materials, data are typically collected in the "water window" (284-543 eV). While the linear absorption coefficient of oxygen in this energy range is small, it is notably higher for other substances. In this range, carbon containing materials absorb strongly, whereas water molecules are mostly transparent. Different carbon functional groups can be detected and imaged when fine-tuning the energy around the carbon $K$-edge.

\subsection{Instrumentation and data acquisition}

6.4.1. Scanning transmission X-ray microscope (STXM) at BESSY II. The experiment was carried out both with the STXM at the undulator beamline U41-STXM (BESSY II, Berlin) [130], and at a laser driven soft X-ray plasma source in the water window based on a pulsed krypton gas target [120]. The design of the STXM (cf. Fig. 6.4.1) is described in detail in [131]. 


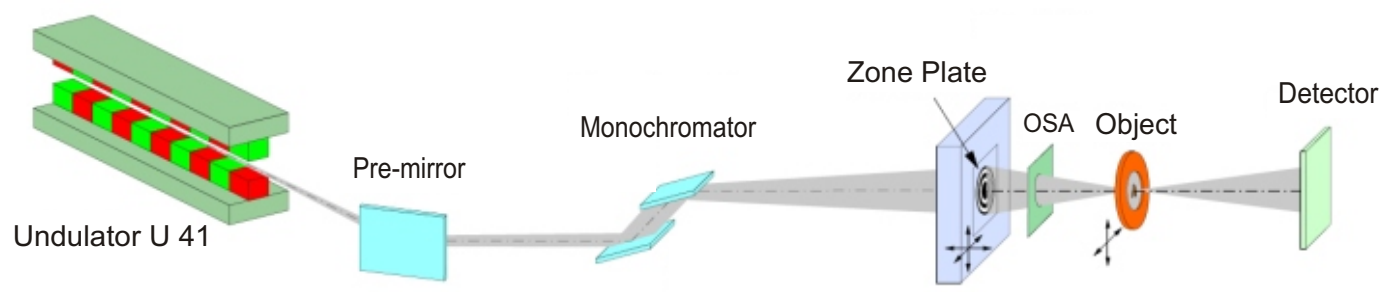

FIGURE 6.4.1. Schematic overview of the BESSY II STXM (see text) [131].

The undulator U41 provides high brilliance X-rays which pass through the plane grating monochromator with varied line density [130] to select a narrow photon energy range. The resulting maximum energy resolution with the STXM is about $0.18 \mathrm{eV}$ at $300 \mathrm{eV}$. A silicon Fresnel zone plate [132] was used as a diffractive lens to form a high resolution focused spot of soft X-rays. The Fresnel zone plate (FZP) used here has a diameter of $200 \mu \mathrm{m}$, the outer most width of $50 \mathrm{~nm}$, the number of zones is 1000 and a central stop of $65 \mu \mathrm{m}$, with a theoretical Rayleigh resolution of $61 \mathrm{~nm}$ in the first diffraction order. An order-sorting aperture of a $40 \mu$ m radius is placed between the sample stage and zone plate. The sample is scanned in the $\mathrm{x}, \mathrm{y}-$ plane by moving the FZP with a high resolution piezo scanner. The transmitted intensity through the sample is recorded on a single pn-CCD unit [133] of 200 pixels x 64 pixels with $150 \times 150 \mu \mathrm{m}^{2}$ pixel size. During experiments, the detector is kept at liquid-nitrogen temperature $(77 \mathrm{~K})$ and vacuum conditions of $3 \times 10^{-8} \mathrm{mbar}$ to reduce the dark current.

Samples were prepared as described in Chapter 3 and glued onto a metal shim. The shim was then fixed between the two specimen chamber rings (see Fig. 6.4.2). The specimen chamber was specifically designed to be used with the scanning transmission X-ray microscope at BESSY II [134].

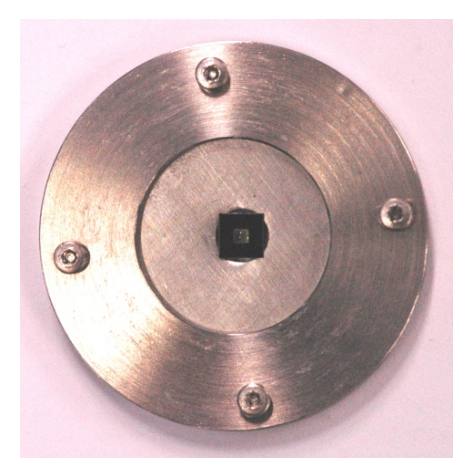

FIGURE 6.4.2. The STXM sample holder. The sample is placed between two silicon nitride windows and these are then glued onto a metal shim. The shim is fixed between the two specimen chamber rings.

6.4.2. Laboratory scale $X U V$ source. The experimental setup for soft $X$-ray radiation emitted from laser plasmas (cf. Fig 6.4.3) consists of a Nd:YAG laser beam (Innolas, $1064 \mathrm{~nm}, 1 \mathrm{~Hz}$, $800 \mathrm{~mJ}, 7 \mathrm{~ns}$ ) focused into a pulsed krypton gas jet centered in a vacuum chamber [135]. 


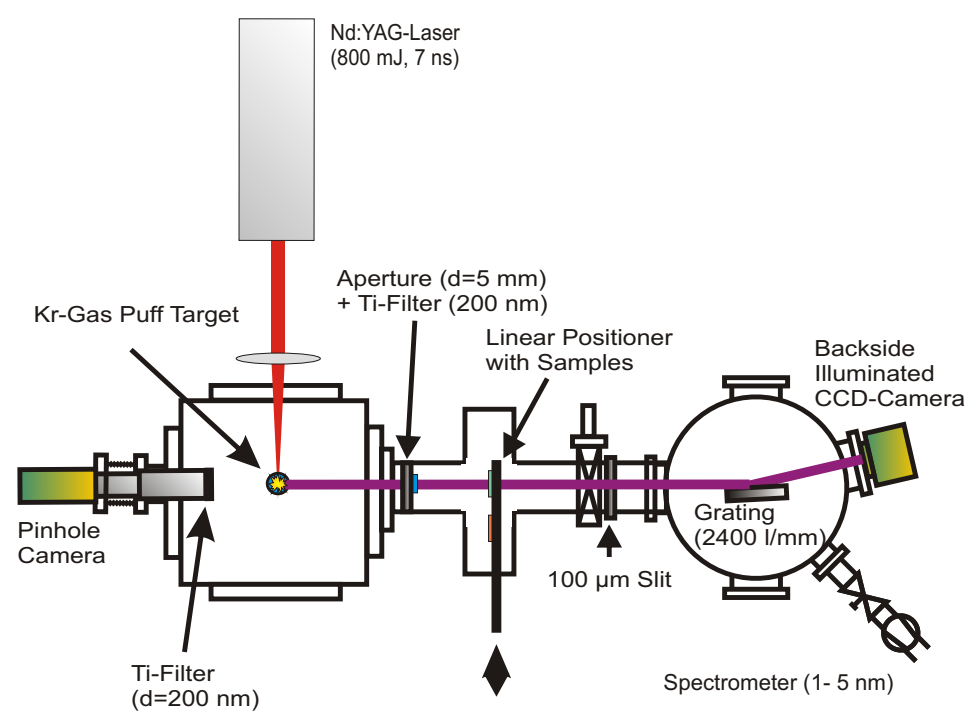

FIGURE 6.4.3. Experimental setup of the laser-plasma XUV source used for NEXAFS experiments.

The laser focus has a diameter of about $60 \mu \mathrm{m}$, yielding power densities of up to $4 \times 10^{12}$ $\mathrm{W} / \mathrm{cm}^{2}$. The size of the krypton plasma is about $250 \mu \mathrm{m}$ (FWHM) in the horizontal and $150 \mu \mathrm{m}$ in the vertical direction. An XUV spectrometer $(1-5 \mathrm{~nm})$ was mounted $90^{\circ}$ to the laser beam. The spectrometer consists of a $100 \mu \mathrm{m}$ entrance slit, an aberration corrected flat-field grating (Hitachi, 2400 lines/mm) and a back-side illuminated CCD camera (Roper Scientific, pixel size $13 \mu \mathrm{m})$. The resolution was $\lambda / \delta \lambda \approx 200$ at $2.87 \mathrm{~nm}$. To block visible radiation from the plasma and scattered laser light a titanium foil (200 $\mu \mathrm{m}$ thickness) was positioned between the plasma source and the sample. Due to the small mean free path of the soft X-ray radiation at atmospheric pressure the target vacuum chamber is evacuated to approx. $10^{-4}$ mbar. For adjustment in the XUV beam the samples were mounted on a rotary/linear motion stage. The distance between the plasma source and the sample is about $220 \mathrm{~mm}$ and the distance between sample and the entrance slit of the spectrometer is about $425 \mathrm{~mm}$.

To obtain NEXAFS-spectra with a high signal-to-noise ratio the data acquisition was accumulated over 1000 pulses (total acquisition time of about $16 \mathrm{~min}$ ). Since it was not possible to record the transmitted intensity through the sample and the reference spectra simultaneously, these were taken consecutively. In previous experiments the stability of the laser plasma source was tested for corresponding acquisition times and it was found that the deviation between the spectra was less then $5 \%$. Since the laser plasma source emits radiation into $4 \pi$ sr the sample was fully illuminated. Nevertheless, only radiation transmitted through an area of $100 \mu \mathrm{m} \times 2.3 \mathrm{~mm}$ of the sample is collected by the spectrometer, since the entrance slit in combination with the detector height is the limiting aperture.

\subsection{Results}

6.5.1. Elemental Contrast Imaging. Using the STXM for absorption difference imaging was performed by taking two images of the same area at either side of the carbon $K$ absorption 
edge, at $E_{1}$ and $E_{2}$, respectively. The carbon map is found by taking the log of the ratio of corresponding pixel counts. This results in an image, which when displayed, indicates increasing carbon concentration by an increasing brightness level [136].

Fig. 6.5.1 shows X-ray micrographs of the wet specimen composed of a supported DPPC/DPPS (4:1) lipid layer in the gel phase binding amidine functionalised polystyrene microspheres with radii of $195 \mathrm{~nm}$. An image of the region of interest below $\left(E_{1}=284 \mathrm{eV}\right.$, top) and above $\left(E_{1}=314\right.$ $\mathrm{eV}$, center) the carbon $K$-edge were taken. The darker the color, the less intense is the measured signal. The quotient image (Fig. 6.5.1, bottom) shows the distribution of carbon. The image of a different region of the sample and magnified images of the selected area taken at $284 \mathrm{eV}$ and $314 \mathrm{eV}$ with the corresponding carbon map image are shown in Fig. 6.5.2 Periodic (moiré) noise in all images is presumably due to aliasing (beating) of a systematic noise signal with the scan frequency of the STXM.
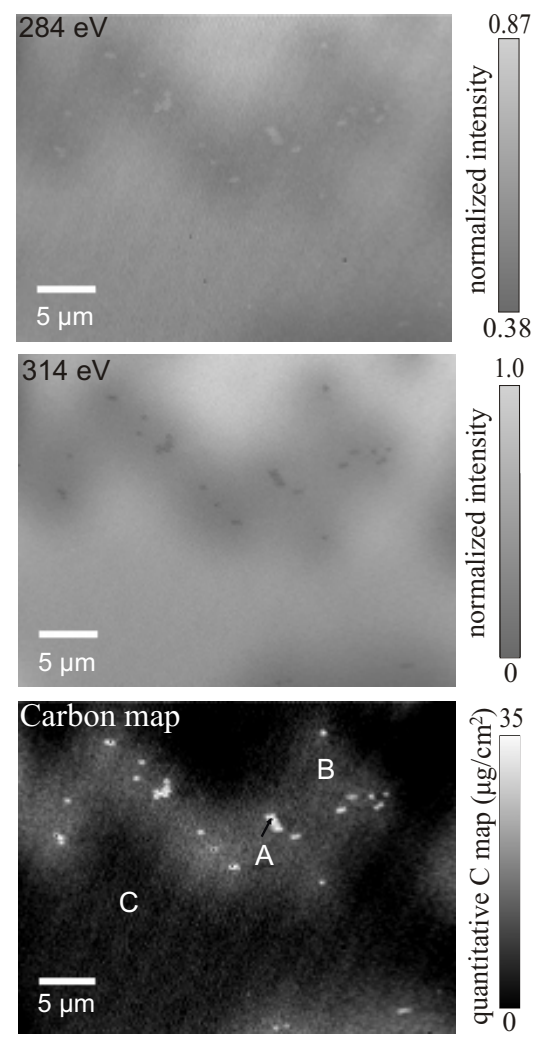

FIGURE 6.5.1. Elemental imaging at the carbon $K$ absorption edge from the lipid layer/PS microspheres sample in the wet state on a $\mathrm{Si}_{3} \mathrm{~N}_{4}$ membrane. Shown are absorption contrast images (a) below the absorption edge at $284 \mathrm{eV}\left(I_{1}\right.$, top) and above the absorption edge at $314 \mathrm{eV}\left(I_{2}\right.$, center). The carbon map (bottom) is calculated from the top and the center image. The gray scale bar indicates the amount of carbon. The arrow ('A') in the carbon map image indicates an amidine coated PS latex microspheres. 'B' indicates a lipid layer. The images have of 37 $\mu \mathrm{m} \times 29 \mu \mathrm{m}$ size, $200 \mathrm{~nm}$ pixel size and the pixel dwell time is $6 \mathrm{~ms}$. The image acquired at $284 \mathrm{eV}$ has a higher relative noise level because of the lower photon rate. 

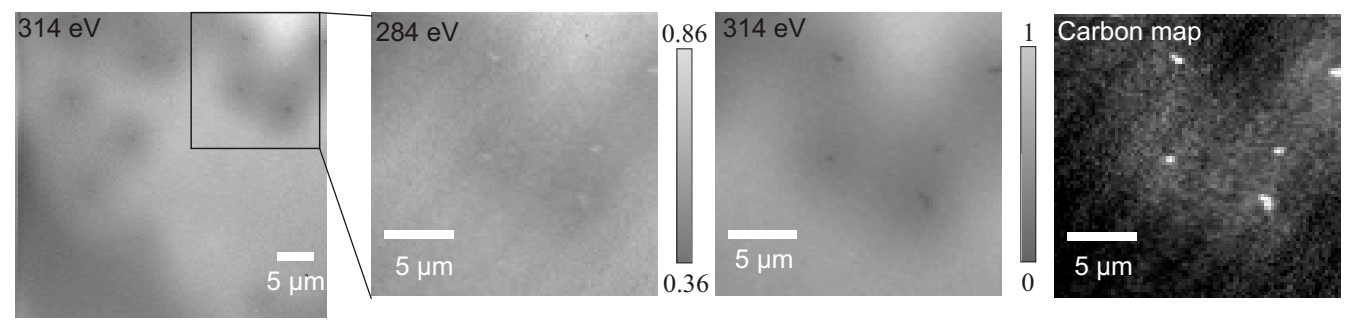

FIGURE 6.5.2. (left) Image of the lipid layer/PS microspheres sample in the wet state. Magnified images of the selected area were taken at $284 \mathrm{eV}$ and $314 \mathrm{eV}$, and the carbon map image was created (right). Images have $18 \mu \mathrm{m} \times 18 \mu \mathrm{m}$ size, $200 \mathrm{~nm}$ pixel size and the pixel dwell time is $6 \mathrm{~ms}$.

Away from an absorption edge, the the linear absorption coefficient has an $E^{-3}$ dependence. Therefore, we can estimate the average thickness of selected pixels in the image corresponding to a certain compound as:

$$
d_{\text {comp }}=\frac{\ln \left(I_{01} / I_{1}\right)-\left(\lambda_{1} / \lambda_{2}\right)^{3} \ln \left(I_{02} / I_{2}\right)}{\mu_{1}-\mu_{2}\left(\lambda_{1} / \lambda_{2}\right)^{3}},
$$

where $I_{01}$ and $I_{02}$ are the intensities of the incident X-ray beams, $I_{1}$ and $I_{2}$ are the intensities of the transmitted X-rays and $\mu_{1}$ and $\mu_{2}$ are the linear absorption coefficients at $E_{1}$ and $E_{2}$, respectively [137].

Pixels corresponding to polystyrene latex microspheres $\left(C_{8} H_{8}\right)$ with the density $1.055 \mathrm{~g} \mathrm{~cm}^{-3}$ were selected (region A in Fig. 6.5.1(c)). Linear absorption coefficients of the polystyrene were calculated from Eq. 6.2 .2 to be $\mu_{P 1}=0.18 \mu \mathrm{m}^{-1}$ and $\mu_{P 2}=4.0 \mu \mathrm{m}^{-1}$ at $E_{1}$ and $E_{2}$, respectively. The thickness of the selected region A was estimated to be $0.33 \pm 0.09 \mu \mathrm{m}$. The error in the measured thickness is $\sim 30 \%$ [136].

Selected region B in Fig. 6.5.1 (c) should corresponds to the DPPC/DPPS (4:1) lipid layer in the gel phase with the density $1.25 \pm 0.08 \mathrm{~g} \mathrm{~cm}^{-3}$. The density was estimated from values of area per lipid in the mixture $\mathrm{A}=43.3 \pm 0.25 \AA^{2}$, thickness of the membrane $\mathrm{d}=45 \pm 0.5 \AA$, and molecular weights $\mathrm{MW}(\mathrm{DPPC})=734.05$ and $\mathrm{MW}(\mathrm{DPPS})=757.96$. Linear absorption coefficients of the lipid mixture according to Eq. 6.2.2 are $\mu_{L 1}=0.49 \mu \mathrm{m}^{-1}$ and $\mu_{L 2}=3.7 \mu \mathrm{m}^{-1}$ at $E_{1}$ and $E_{2}$, respectively. The thickness of the region B was calculated to be $14 \pm 4.2 \mathrm{~nm}$.

The water thickness in the specimen was estimated from the region $\mathrm{C}$ in Fig. 6.5.1 (c). The flux $I_{0}$ behind two $\mathrm{Si}_{3} \mathrm{~N}_{4}$ foils without specimen, at the pre-edge energy $284 \mathrm{eV}$, was $5.4 \times 10^{5}$ photons $/ 6 \mathrm{~ms}$. The flux $I$ behind the wet specimen sandwiched between two $\mathrm{Si}_{3} \mathrm{~N}_{4}$ foils in the region $\mathrm{C}$, at $284 \mathrm{eV}$, was $4.3 \times 10^{5}$ photons $/ 6 \mathrm{~ms}$. The water thickness was calculated from $t_{\text {water }}=-\ln \left(I / I_{0}\right) / \mu_{\text {water }}$ to be $430 \mathrm{~nm}$.

Gray scale bar in Fig 6.5.1 (c) indicates the carbon mass density $m_{C}=\rho_{C} d_{C}$, where the carbon density $\rho_{C}$ is assumed to be $1.05 \mathrm{~g} \mathrm{~cm}^{-3}$ and $d_{C}$ is calculated using Eq. 6.5.1.

6.5.2. Spectromicroscopy of dried amidine functionalized polystyrene particles. A method to obtain spectromicroscopic data, using STXM, is to acquire an image sequence over a series of spectroscopically interesting energies [138]. The images in this sequence can than be 
correlated to each other and shifted to provide the proper alignment. A spectrum is obtained from each selected object point by plotting the optical density (OD) of all energies whose images have been taken. In this way, an image is obtained at each energy, and also a spectrum over the entire energy range can be extracted from each pixel or from larger regions. A beam shutter was used to block the beam during energy changes. Refocusing after a change in energy was necessary.

The measurement was performed on air-dried amidine functionalized PS microspheres with radii of $195 \mathrm{~nm}$ deposited on the $\mathrm{Si}_{3} \mathrm{~N}_{4}$ membrane. A sequence of $72 \mathrm{X}$-ray micrographs (10 $\mu \mathrm{m} \mathrm{x}$ $10 \mu \mathrm{m})$ at a spatial resolution of $100 \mathrm{~nm}$ per pixel ( $6 \mathrm{~ms}$ dwell time per pixel) with energy steps of $0.25 \mathrm{eV}$ over the energy range 278 to $296 \mathrm{eV}$ were taken. The images in the image sequence were aligned with each other and the PS spectrum from the region of interest was extracted by using a package of stack analyze routines, developed by the group of C. Jacobsen [138].

The presence of PS microspheres was revealed through a change in contrast, e.i. high transmission at $278 \mathrm{eV}$ and strong absorption at $285 \mathrm{eV}$ (Fig. 6.5.2 (a)) corresponding to the $\pi^{*}(C=C)$ resonance.

In the carbon map, the distribution of organic carbon (last image in Fig. 6.5.2 (a)) was obtained by taking the ratio of images acquired at different energies (278-283 vs. 290-296 eV) and mapping out the areas that exhibited the greatest change in absorbance just below the carbon absorption edge $(290 \mathrm{eV})$.

To extract the spectrum of PS microspheres from the image sequence, we selected pixels corresponding to the particles, $I$ region. The necessary incident flux $I_{0}$ was derived from the region in the images without object structure (see Fig. 6.5.2 (b)). The spectrum shown in Fig. 6.5.2 (c) was calculated as $O D=-\ln \left(I / I_{0}\right)$ and plotted as a function of energy. The Specfit package [139] was used to apply the Gaussian fitting for a more accurate determining of peak positions and an arctangent stepfunction at $290.1 \mathrm{eV}$ for the ionization continuum onset. The instrument was calibrated using the well known spectrum of $\mathrm{CO}_{2}$.

The spectrum of PS microspheres has a single, very intense peak at $285.1 \mathrm{eV}$, due to the $\pi^{*}(C=C)$ resonance. The feature near $287.3 \mathrm{eV}$ is typically assigned to a Rydberg transition and the $288.9 \mathrm{eV}$ feature to a $\mathrm{C} 1 s(C-H) \rightarrow 2 \pi^{*}(C=C)$ transition. Peak positions correspond well to NEXAFS data obtained from poly $(\alpha$-methyl styrene) by Dhez et al. [140]. The shoulder at 285.9 $\mathrm{eV}$ (2) might correspond to the $\mathrm{C} 1 s(C-N) \rightarrow 1 \pi^{*}(C=C)$ transition in amidine.

6.5.3. Spectromicroscopy of the lipid layer with polystyrene microspheres. Wet sample of a supported DPPC/DPPS (4:1) lipid layer in the gel phase binding amidine functionalized PS microspheres with radii of $195 \mathrm{~nm}$, prepared between two $\mathrm{Si}_{3} \mathrm{~N}_{4}$ membranes, was observed by STXM. An image sequence of $7 \mu \mathrm{m} \times 7 \mu \mathrm{m} X$-ray micrographs at a spatial resolution of $70 \mathrm{~nm}$ per pixel (6 ms dwell time per pixel) with energy steps of $0.25 \mathrm{eV}$ over the energy range 278 to $297 \mathrm{eV}$ was acquired.

Fig. 6.5.4 (a) shows selected images from the sequence. Lighter shades correspond to a higher transparency. The absorption contrast reversal is clearly revealed. For example, at the pre-edge energy $281.2 \mathrm{eV}$, water absorbs stronger than PS microspheres, hence they appear lighter at this energy. Strong absorption of PS spheres at $285 \mathrm{eV}$ corresponds to the $\pi^{*}(C=C)$ resonance in PS. 

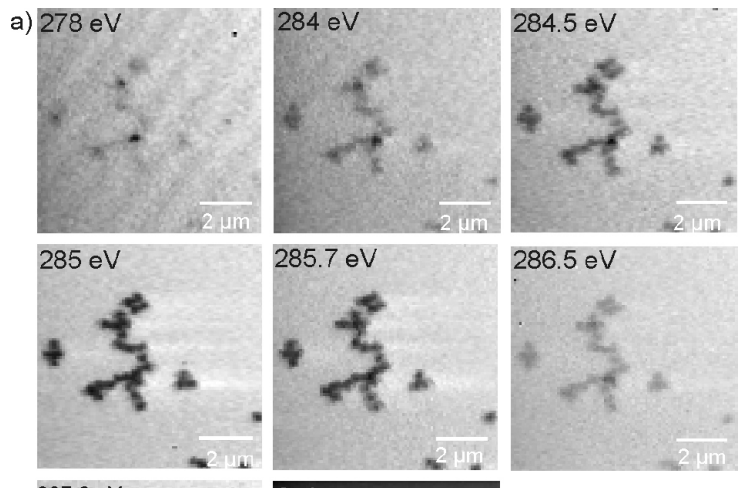

$285.7 \mathrm{eV}$

$286.5 \mathrm{eV}$
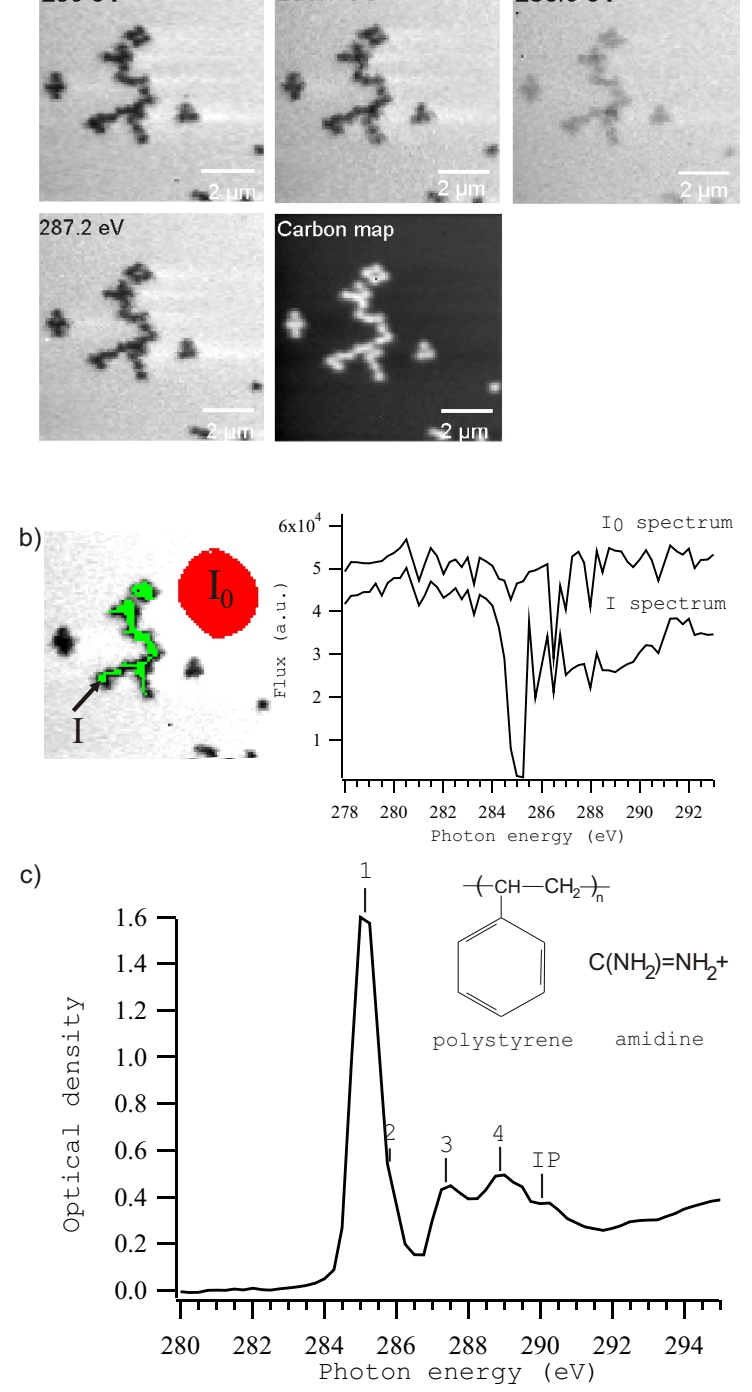

FIGURE 6.5.3. a) STXM images of the dried amidine PS particles sample at selected photon energies. b) (left) Selected regions for transmitted X-ray intensity $I$ (green), incident X-ray intensity $I_{0}$, and corresponding $I$ and $I_{0}$ intensities plotted as a function of photon energy (right). c) The derived spectrum $\left(O D=-\ln \left(I / I_{0}\right)\right)$ at the carbon $K$-edge. The features of the spectrum are explained in the text.

The carbon map (Fig. 6.5.4 (a), last image) obtained from the image sequence clearly shows lipid layer regions (region B). Lighter shades correspond to higher carbon concentrations.

Fig. 6.5.4 (b,c) present the spectra extracted from spatially selected regions 'A' (microspheres) and ' $\mathrm{B}$ ' (the lipid layer). The spectrum from the region A is similar to that of dried PS, except for an additional peak at $287.8 \mathrm{eV}$. This peak might be the $\pi^{*}(C=O)$ resonance in the underlying 


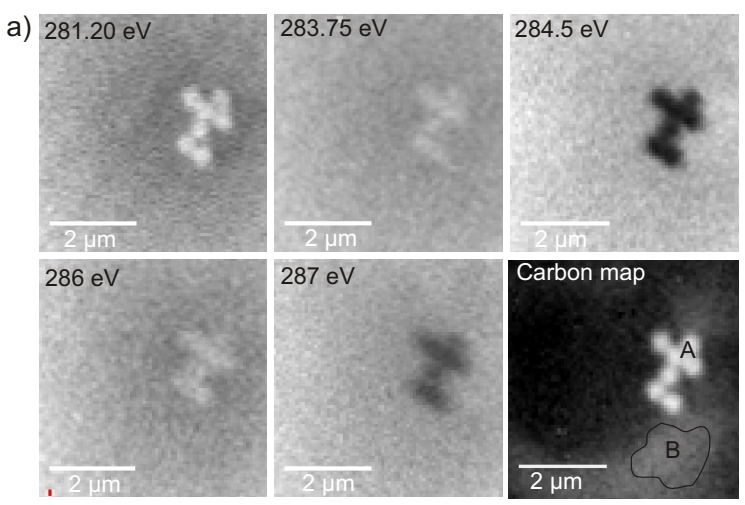

b)

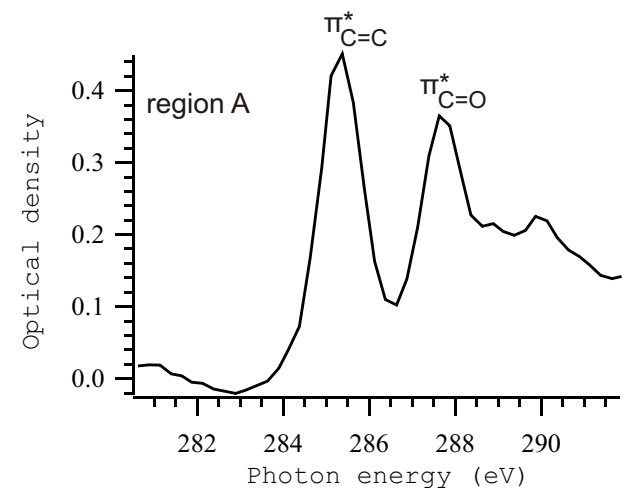

c)

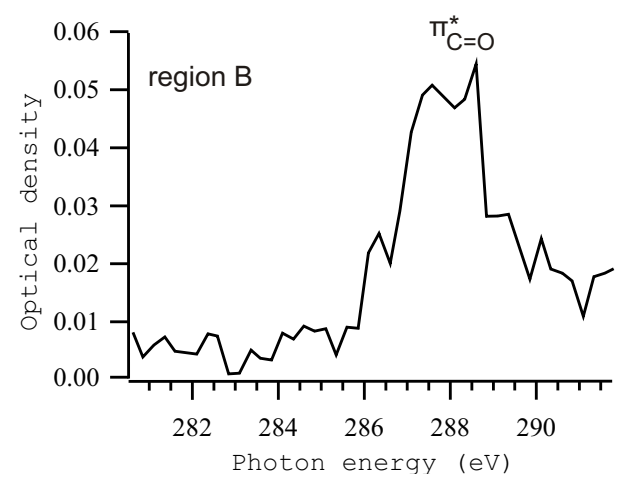

FIGURE 6.5.4. a) X-ray micrographs of the supported DPPC/DPPS (4:1) lipid layer in the gel phase binding amidine-PS microspheres at selected photon energies and the resulting carbon map image. b,c) Spectra from spatially selected regions, corresponding to particles and the lipid layer.

lipid layer. The spectrum from the region B shows a readily differentiated peak at $287.9 \mathrm{eV}$ (the $\mathrm{C} 1 s(C=O) \rightarrow \pi^{*}(C=O)$ transition) typical of lipids.

The overlayer of buffer is estimated from the pre-edge signal to be app. $520 \mathrm{~nm}$ thick. Optical density associated with the lipid signal in the region $\mathrm{B}$ is only $\sim 0.02$ in the continuum. This is equivalent to a lipid layer thickness of $5.5 \pm 2.7 \mathrm{~nm}$. The uncertainties in the quantification of the weak signal from the surface adsorbed organic layer is rather high - perhaps as much as $50 \%$ [124]. Together these results constitute clear evidence that scanning transmission X-ray microscopy can detect a supported lipid layer in the aqueous environment. 
In scanning transmission X-ray microscopy the dosage applied to the specimen and the associated radiation damage, especially of biological specimen, plays an important role. Irradiation is generating broken chemical bonds in biological structures by excitations and more likely by ionizations of molecules. According to [141] we can estimate the radiation dose in Gray as:

$$
\text { dose }=1.602 \times 10^{-4} \frac{E N t \mu}{A \rho \eta},
$$

where $N$ is number of absorbed photons per msec with the photon energy $E$ in $\mathrm{eV}$ and $t$ is the exposure time in msec. $A$ represents the size of radiated area in $\mu \mathrm{m}^{2}$ and $\rho$ is the density in $\mathrm{g} / \mathrm{cm}^{3}$. $\mu$ is the linear absorption coefficient in $\mu m^{-1}$ and $\eta$ is the detector efficiency (52\% in the $\mathrm{C} 1 \mathrm{~s}$ region [131]).

With a pixel size of $70 \mathrm{~nm}, 6 \mathrm{msec}$ exposure time and $1.25 \mathrm{~g} / \mathrm{cm}^{3}$ density of lipids we calculated the radiation dose per pixel, applied during a 56 energy image sequence, to be $\sim 1.2 \times$ $10^{7} \mathrm{~Gy}$. With a pixel size of $100 \mathrm{~nm}, 6 \mathrm{msec}$ exposure time, $1.055 \mathrm{~g} / \mathrm{cm}^{3}$ density of polystyrene particles the radiation dose applied during an image sequence (74 images) becomes $\sim 4.6 \times 10^{7}$ Gy. In a study of PMMA, an organic polymer used as a photoresist, $50 \%$ mass loss was measured at a dose of $10^{7} \mathrm{~Gy}[\mathbf{1 4 2}]$. At least partial radiation damage of specimen during an acquisition must therefore be considered when taking image sequences (image stacks).

6.5.4. NEXAFS spectra of phospholipid multilayers. Dried lipid multilayers on $\mathrm{Si}_{3} \mathrm{~N}_{4}$ membranes were prepared as described in the chap. 4 . Fig. 3.6 in the chap. 4 shows the image of a DMPC multilayer sample obtained by a optical imaging profiler. The thickness of multilayers in this sample was measured to be $\sim 120 \mathrm{~nm}$.

NEXAFS measurement were obtained by using a laboratory scale setup based on a laserdriven plasma source. The resolution of this spectrometer was experimentally determined to be $\lambda / \Delta \lambda \approx 200$ at $2.87 \mathrm{~nm}$. Nitrogen was used as target gas to calibrate the spectrometer. The emission spectra of the krypton plasma both with and without the sample were acquired, each obtained by an integration over 1000 pulses (total acquisition time about $16 \mathrm{~min}$ ). The optical density was evaluated according to $O D=-\ln \left(I / I_{0}\right)$ and plotted as a function of energy.

The NEXAFS spectra of DMPC, DOPS and DOPC lipid multilayers are displayed in Fig. 6.5.5 and 6.5.6. When approaching an edge by tuning the energy from the lower end to the edge, the $\pi^{*}$ resonances are typically show first, followed by Rydberg and/or hydrogen-derived resonances, and above the ionization potential, $\sigma^{*}$ resonances are present. The energies and proposed assignments are listed in Tab. 1 .

The spectrum of the saturated DMPC (Fig. 6.5.5) has three distinct spectral features: The $288.7 \mathrm{eV}$ peak might correspond to the $\pi^{*}(C=O)$ resonance of the ester carbonyl group. A broad maximum at $292.2 \mathrm{eV}$ might be attributed to $\sigma^{*}(C-C)$ resonance. At $294.5 \mathrm{eV}$ the $\sigma^{*}$ feature can be assigned to the $\mathrm{C}-\mathrm{O}$ bond of the carboxylate group $\left(-\mathrm{CO}_{2}-\right)$.

Spectra of unsaturated DOPS and DOPC (Fig. 6.5.6) exhibit relatively strong, sharp peak at $285.1 \mathrm{eV}$ and $285 \mathrm{eV}$ respectively, assigned to the $\pi^{*}(C=C)$ resonance. A shoulder at $288 \mathrm{eV}$ in the spectrum of the DOPS and at $287.7 \mathrm{eV}$ in the spectrum of the DOPC might correspond to the $\pi^{*}(C=O)$ resonance of the ester carbonyl group. The Rydberg/C- $\mathrm{H}^{*}$ resonances might 
TABLE 1. Energies and assignments of spectral features in the NEXAFS spectra of DMPC, DOPC and DOPS.

\begin{tabular}{ccccc}
\hline Feature & \multicolumn{2}{c}{ Energy $(\mathrm{eV})$} & Assignment \\
& DMPC & DOPC & DOPS & \\
\hline 1 & & 285.1 & 285.0 & $1 \pi^{*}(C=C)$ \\
2 & 288.7 & 287.7 & 288.0 & $\pi^{*}(C=O)$ \\
3 & & 289.4 & 289.5 & $\mathrm{Ryd} . / \mathrm{C}-\mathrm{H}^{*}$ \\
4 & 292.2 & 293.1 & 292.8 & $\sigma^{*}(C-C)$ \\
5 & 294.5 & 295.6 & 295.8 & $\sigma^{*}(C-O)$ \\
6 & & & 298.7 & $\sigma^{*}(C=O)$ \\
7 & & 302.8 & 302.9 & $\sigma^{*}(C=C)$ \\
& 290.8 & 291.7 & 291.5 & IP \\
\hline
\end{tabular}

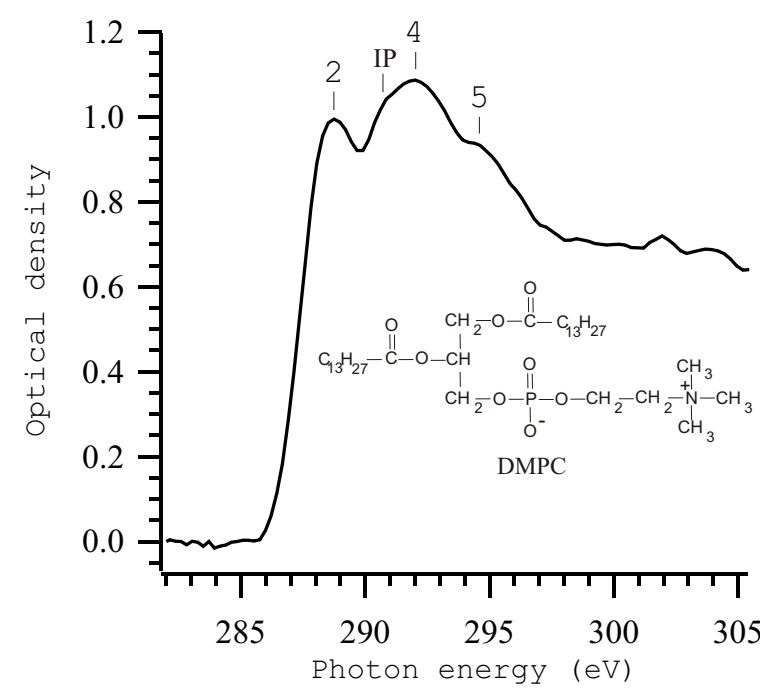

FIGURE 6.5.5. NEXAFS spectra of the saturated DMPC phospholipid layer. Lines indicate locations of peaks and C1s IPs as estimated.

be present at $289.4 \mathrm{eV}$ in the DOPC spectrum. The peak at $289.5 \mathrm{eV}$ in the DOPS spectrum is more pronounced and can be assigned to the overlap of the Rydberg/C- $\mathrm{H}^{*}$ resonances and $\mathrm{C} 1 s \rightarrow$ $\sigma^{*}(\mathrm{C}-\mathrm{NH})$ transition in the $-\mathrm{CNH}_{3}^{+}$group of the serine. The broad maxima (features 4 and $5)$ in the continuum of both spectra are attributed to the $\sigma^{*}(C-C)$ and $\sigma^{*}(C-O)$ resonances, respectively. The $302.8 \mathrm{eV}$ peak in the DOPC spectrum and the $302.9 \mathrm{eV}$ peak in the DOPS spectrum might be $\sigma^{*}(C=C)$ resonance. The DOPS spectrum contains the additional small peak at $298.7 \mathrm{eV}$ (feature 6), which is probably a $\sigma^{*}(C=O)$ resonance in the carbonyl (-COO${ }^{-}$) functional group. Features at $\sim 310 \mathrm{eV}$ cannot be attributed to the sample. We have checked that the features do not occur systematically in all spectra taken with this instrument by comparing our spectrum with spectra taken from other samples.

These assignments were made on the basis of inner-shell spectra for related compounds, as complex alcohols [18], carboxylic acids [143] and amino acids [144].

We have tested the sensitivity of the NEXAFS measurement depending on the lipid film thickness. Fig. 6.5.7 shows series of NEXAFS spectra in transmission of DMPC multilayers of different 


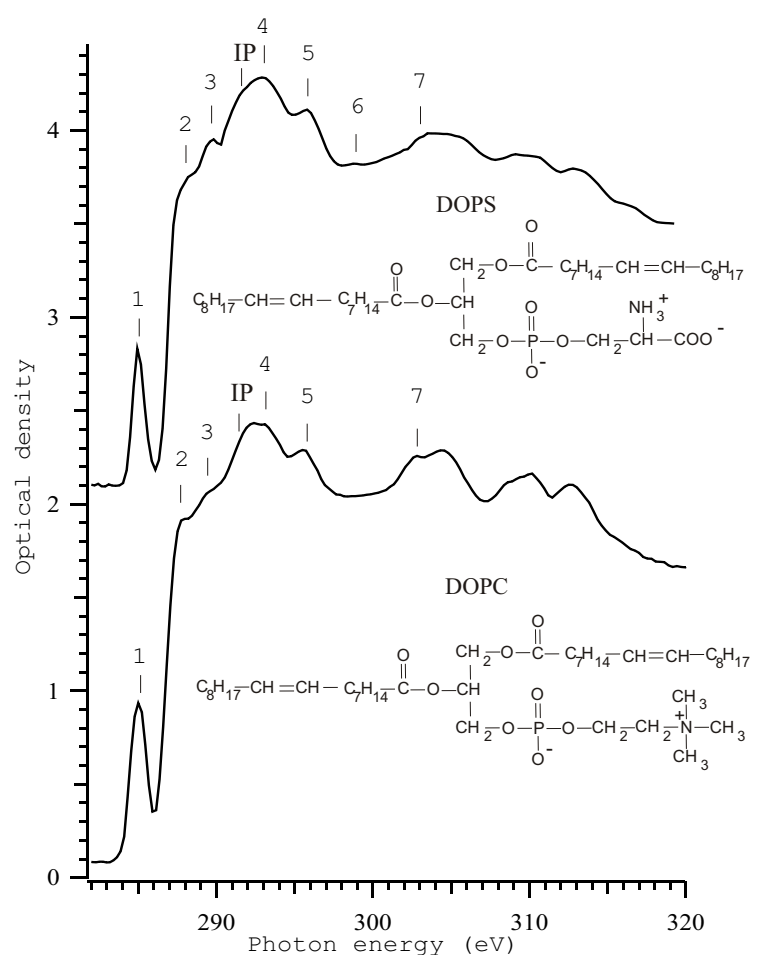

FIGURE 6.5.6. NEXAFS spectra of unsaturated DOPS and DOPC phospholipids. Lines indicate locations of peaks and the IP as estimated. The spectra are shifted vertically for clarity.

thickness. Thicknesses were estimated from optical densities. The $\pi^{*}(C=O)$ resonance from $\sim$ $37 \mathrm{~nm}$ thick layer can be still resolved.

\subsection{Summary and Conclusion}

In summary, we have successfully used scanning transmission X-ray spectro-microscopy to image positively charged polystyrene latex microspheres binding to a supported two-component lipid membrane, composed of charged and neutral lipid species. At the given parameters of vesicle fusion (i.e. in the absence of added calcium ions), the coverage was not complete, as expected. The presence of bilayer patches enabled us to investigate the sensitivity of scanning transmission $\mathrm{X}$-ray spectro-microscopy down to the level of a thin lipid layer. However, current experimental conditions are at the limit of a sufficient signal-to-noise ratio, resulting in rather diffuse patch boundaries in the image. Nevertheless, preferential binding of microspheres to the oppositely charged bilayer was observed. Surprisingly, the bound micropheres aggregated on the lipid bilayer patch, despite equal charges and inter-particle repulsion. This finding could be explained by partial neutralization of the charges on the amidinated positively-charged hydrophobic latex surfaces what makes the microspheres coagulated by van der Waals and hydrophobic interactions. This is a clear demonstration of the rich and complex effects which can be observed in these multi-component systems, where the degrees of freedom of the lipid composition as well as the lipid and colloid counterions has to be taken into account. For a clear-cut explanation, more data taken over an 


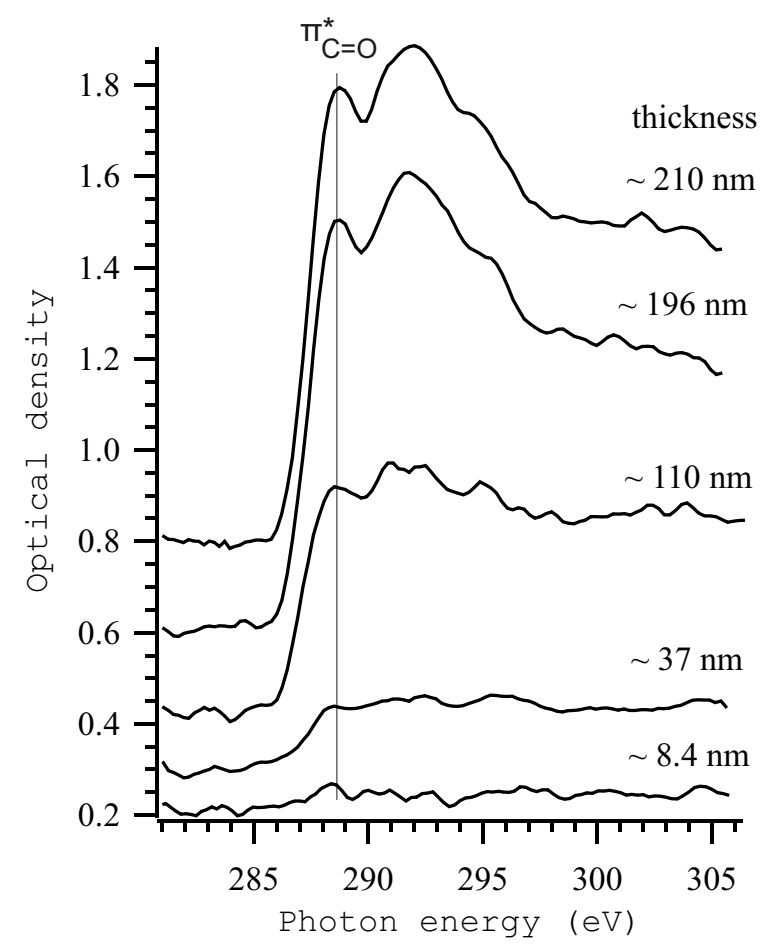

FIGURE 6.5.7. Series of NEXAFS spectra of DMPC multilayers of different thickness. The $\pi^{*}(C=O)$ resonance from $\sim 37 \mathrm{~nm}$ thick layer can be still resolved.

extended parameter range of ionic strength and surface charges needs to be collected. While large microspheres as well as smaller fluorescence markers can be used in fluorescence microscopy to monitor the two-dimensional distribution of bound colloids at the lipid bilayer, the state of the lipid bilayer itself is more difficult to probe. This concerns in particular the degrees of freedom associated with demixing of charged and uncharged species, on the relevant sub-micron length scales. Here synchrotron-based spectro-microscopy has the potential to chemically identify the individual species and to provide compositional maps. As a first step, we present a small data bank of the NEXAFS spectra of some of the most common lipids. Note that the spectroscopic data alone, without spatial resolution, can be collected on compact laser-driven plasma sources, as demonstrated here for the first time. Thereby, lipid NEXAFS spectroscopy is in principle amenable to a much larger number of laboratories, at least to the extent that such instrumentation becomes commercially available. However, it should be pointed out that high resolution data for organic molecules obtained with synchrotron sources contain valuable information, e.g. on vibrational fine structure [18]. At the same time spectro-microscopy is likely to remain the realm of studies using synchrotron radiation. More strongly stated, even today, high resolution compositional maps of single bilayer systems are at the technical limit, but can be expected to benefit significantly from improvements of sources and beamline instrumentation. Therefore, the resolution and sensitivity limits of spectro-microscopy can be expected to shift in the future from a signal-to-noise related issue to an issue of dose-dependent radiation damage. 
We have also presented NEXAFS results obtained by using a laboratory scale (compact) laser driven plasma source. The carbon $K$-edge NEXAFS spectra of selected lipids, which differ in the headgroup structure or/and in containing the $\mathrm{C}=\mathrm{C}$ double bond in hydrocarbon tails have been measured to provide a first step towards reference NEXAFS spectra of different lipids. As demonstrated here, soft X-ray NEXAFS can be carried out by compact instrumentation, owing to the high peak flux, the broad emission spectrum and the high dispersive power of the analyzing grating. 



\section{CHAPTER 7}

\section{Interaction of two-component anionic lipid membrane with gold particles}

\subsection{Introduction}

Since most biomembranes are negatively charged, electrostatics play an important role in interactions of lipid membranes and charged macromolecules. In model experiments, membrane inclusions can be mimicked by nanoscopic colloidal particles bound to lipid membranes [70, 145].

Electrostatic interactions between a charged nanoparticle and a charged low dielectric membrane surface in an electrolyte solution is a complex problem. The reason for this is that it accounts for three body mutual interaction of surface-nanoparticle-electrolyte triple. Furthermore, lipid membranes are not static, flat homogeneous structures, but present a large number of dynamic modes. Individual lipid molecules in a bilayer undergo lateral diffusion, wobbling, rotations, and vertical excursions (protrusions) out of the bilayer [1] . This naturally has an impact on the structure of the bilayer itself as a structured interface with specific elastic and mechanical properties.

When a membrane is in a fluid state, the lipids have a relatively fast lateral diffusion and are, in principle, responsive when a charged object approaches. Lipid domain formation (demixing) of the lipids has been observed experimentally upon adsorption of DNA molecules on cationic membranes [146] and adsorption of peptides on giant unilamellar vesicles [147]. The number of theoretical and computational studies on interactions of proteins [17, 148, 149], polyectrolytes [150] and charged colloids [151] with fluid lipid membranes has increased in the recent years. They have confirmed that the adsorption of macromolecules induces demixing of lipids in the membrane and that the demixing, in turn, increases the binding energy of macromolecules to the membrane. In addition, it is known that ion concentration and potential, which can directly influence colloid-membrane interaction, may differ drastically near the membrane compared to the bulk. In order to control this interaction, it is therefore necessary to well-define electrostatic conditions at the interfacial area [152].

Colloids are able to induce local deformations of a flat fluid membrane they adhere to, and this may give rise to various phenomena such as membrane-mediated elastic interactions between colloids [70] or wrapping of the membrane around the colloid [17, 69]. The theoretical study of Fleck and Netz [153] involves bending of the membrane in a presence of colloids and effects of salt, colloid size and charge and membrane charge density. Furthermore, wrapping of a colloid by a membrane is related to budding of vesicles, which was subject to many studies as well [154, 155, 71].

The aim of this work was to study how fluid and gel membranes carrying $80 \%$ of neutral and $20 \%$ negatively charged lipids, respond to interaction with positively charged gold nanoparticles 
in buffers with different $\mathrm{pH}$ and ionic strength. Supported DOPC/DOPC and DPPC/DPPS lipid bilayers and giant unilamellar vesicles are chosen as model membranes. First, polyelectrolytescoated gold nanoparticles are characterized by UV-vis spectroscopy and scanning transmission microscopy. To determine the contribution to the membrane charge from phosphatidylserine (PS), one must know its intrinsic $p K$, namely the surface $\mathrm{pH}$ at which one-half of PS molecules in the membrane are charged. We have used FT-IR spectroscopy to determine the apparent $p K_{a}$ of the carboxyl group of PS and this value was used to calculate the intrinsic $p K$.

$\mathrm{X}$-ray reflectivity and fluorescence microscopy was used to study supported lipid membranenanoparticle systems and phase contrast microscopy to image the giant unilamellar vesicles interacting with nanoparticles. Results are discussed and compared with made theoretical estimations.

\subsection{Characterization of PSS/PAH coated gold particles}

Preparation of polyelectrolytes-coated gold nanoparticles (AuNPs) involves initial coating of cetyltrimethylammonium bromide (CTAB) stabilized nanoparticles ( $\zeta$ potential, $\zeta \sim+21 \mathrm{mV}$ ) with a negatively charged poly(styrene sulfonate) polyelectrolyte (PSS), resulting in charge reversal ( $\zeta \sim-39 \mathrm{mV}$ ). Then, the adsorption of a second, positively charged poly(allylamine) hydrochloride (PAH) layer subsequently reverses surface charge $(\zeta \sim+40 \mathrm{mV})$. The thickness of each layer is between 1 and $2 \mathrm{~nm}[\mathbf{1 5 6}]$ (see Fig. 7.2.1].
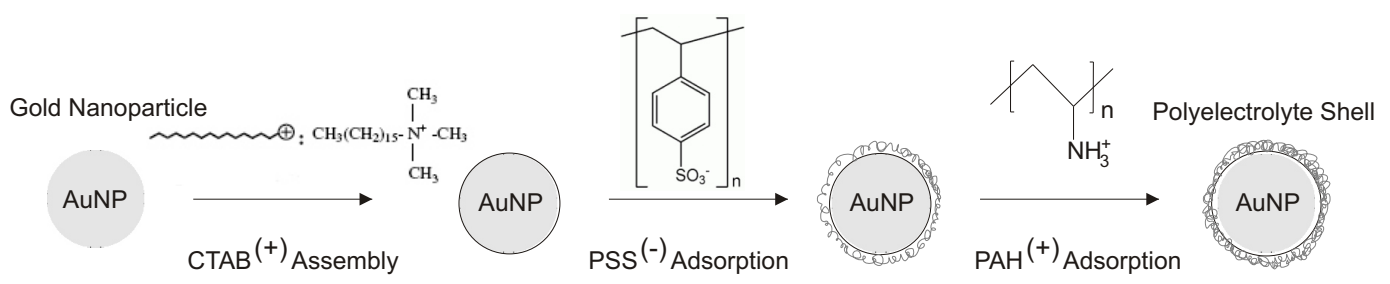

FIGURE 7.2.1. Schematic diagram illustrating the layer-by-layer polyelectrolyte deposition process applied to gold nanoparticles.

Most of the nanoparticle-membrane experiments were carried out in salt buffer solutions. In order to test stability of the particles in strong ionic solutions, UV-visible spectra of the AuNPs in $100 \mathrm{mM} \mathrm{NaCl}$ solution at $\mathrm{pH} 7.4$ and $\mathrm{pH} 4$ were measured. Strong absorption observed at $540 \mathrm{~nm}$ (see Fig. 7.2.2) is due to the surface plasmon resonance of $64 \mathrm{~nm}$ sized AuNPs [157]. The second broad peak in the region of $650-800 \mathrm{~nm}$ [157] which could corresponds to aggregated particles was not observed.

Scanning electron microscopy was carried out to visualize the AuNPs and determine the particle size. The scanning electron microscopy image of bare nanoparticles is shown in Fig. 7.2.2. The AuNPs are spherical in nature and they have size of $64 \pm 5 \mathrm{~nm}$.

\subsection{Dissociation constant of phosphatidylserine}

FT-IR spectroscopy was used to estimate the apparent $p K_{a}$ of carboxylate group of phosphatidylserine. The apparent $p K_{a}$ for a single site group is determined as the bulk $\mathrm{pH}$ at which 50 $\%$ of the group population is charged [158]. The multilamellar DOPS stacks were deposited onto 


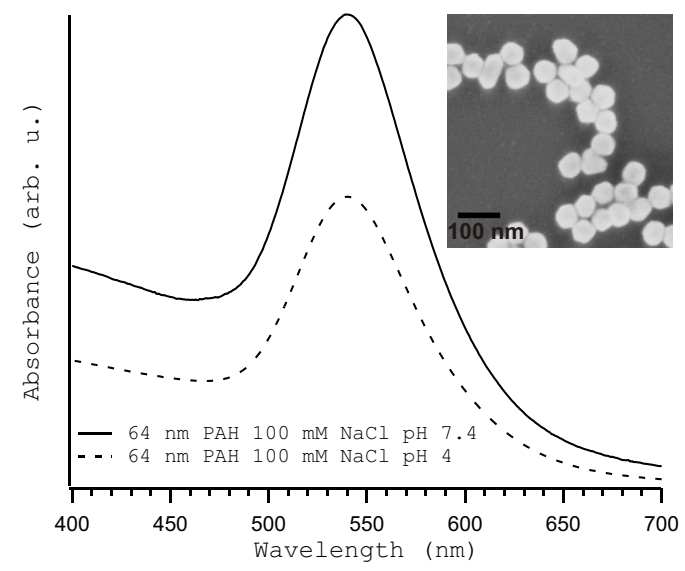

FIGURE 7.2.2. UV-visible absorbance spectra of $64 \mathrm{~nm}$-sized polyelectrolytescoated gold nanoparticles in HEPES buffer with $\mathrm{pH} 7.4$, containing $100 \mathrm{mM} \mathrm{NaCl}$ (solid line) and acetic acid buffer with $\mathrm{pH} 4$ containing $100 \mathrm{mM} \mathrm{NaCl}$ (dashed line). These spectra indicate no aggregation of the particles (see text for explanation). Inset shows the scanning transmission microscopy image of the dried nanoparticles.

well-leveled and cleaned $\mathrm{CaF}_{2}$ substrate as described in Chapter3. Stacks were hydrated with the respective buffer solution and capped by a second $\mathrm{CaF}_{2}$ substrate. Prepared samples were sealed with Parafilm to form a wet cell. A typical FT-IR spectrum of hydrated DOPS is shown in Fig. 7.3.1. Several important peaks were identified and summarized in Tab. 1 .

The spectrum shows typical symmetric and antisymmetric $\mathrm{CH}_{2}$ stretching modes of the oleoyl chains at about $2853 \mathrm{~cm}^{-1}$ and $2924 \mathrm{~cm}^{-1}$, respectively. The minor band that appears at around $2957 \mathrm{~cm}^{-1}$ is due to the asymmetric stretching vibrations of the terminal $\mathrm{CH}_{3}$ groups [159]. Other methylene vibrational modes, such as scissoring absorption bands $\delta\left(\mathrm{CH}_{2}\right)$ appear around 1415 $\mathrm{cm}^{-1}$ and $1466 \mathrm{~cm}^{-1}[\mathbf{1 6 0}] . \mathrm{CH}_{3}$ symmetric bend can be observed at about $1377 \mathrm{~cm}^{-1}$ [161]

The most intense and useful bands for probing the interfacial region of the membrane lipids are the $\mathrm{C}=\mathrm{O}$ stretching bands, which appear in the case of protonated carboxyl groups around 1729 $\mathrm{cm}^{-1}$, and in the case of unprotonated carboxyl groups at about $1642 \mathrm{~cm}^{-1}$ [159, 162]. Symmetric $\mathrm{PO}_{2}^{-}$stretching vibrations are present at $1050 \mathrm{~cm}^{-1}, 1063 \mathrm{~cm}^{-1}$ and $1090 \mathrm{~cm}^{-1}$, respectively, and the asymmetric $\mathrm{PO}_{2}^{-}$stretching band at around $1222 \mathrm{~cm}^{-1}$ [159]. Other bands pertaining to the phospholipid head-group of lipids are less intense or less characteristic.

In order to estimate the apparent $p K_{a}$ of the carboxyl group of DOPS, intensities of the antisymmetric stretching vibration bands of $\mathrm{COO}^{-}$and $\mathrm{COOH}$ were monitored as bulk $\mathrm{pH}$ was changed [162]. The $\mathrm{COO}^{-}$antisymmetric stretching vibration of DOPS appears at $\sim 1642 \mathrm{~cm}^{-1}$ whereas the $\mathrm{COOH}$ band appears at $\sim 1729 \mathrm{~cm}^{-1}$. As the $\mathrm{pH}$ was increased the band corresponding to $\mathrm{COOH}$ group of DOPS decreased in intensity, whereas at the same time the band corresponding to the $\mathrm{COO}^{-}$increased in intensity, as is shown in the Fig. 7.3.2 (a). The apparent $p K_{a}$ of the carboxyl group was obtained, according to the Henderson-Hasselbach equation:

$$
\log \left(\left[A^{-}\right] /[A H]\right)=-p K_{a}+p H
$$




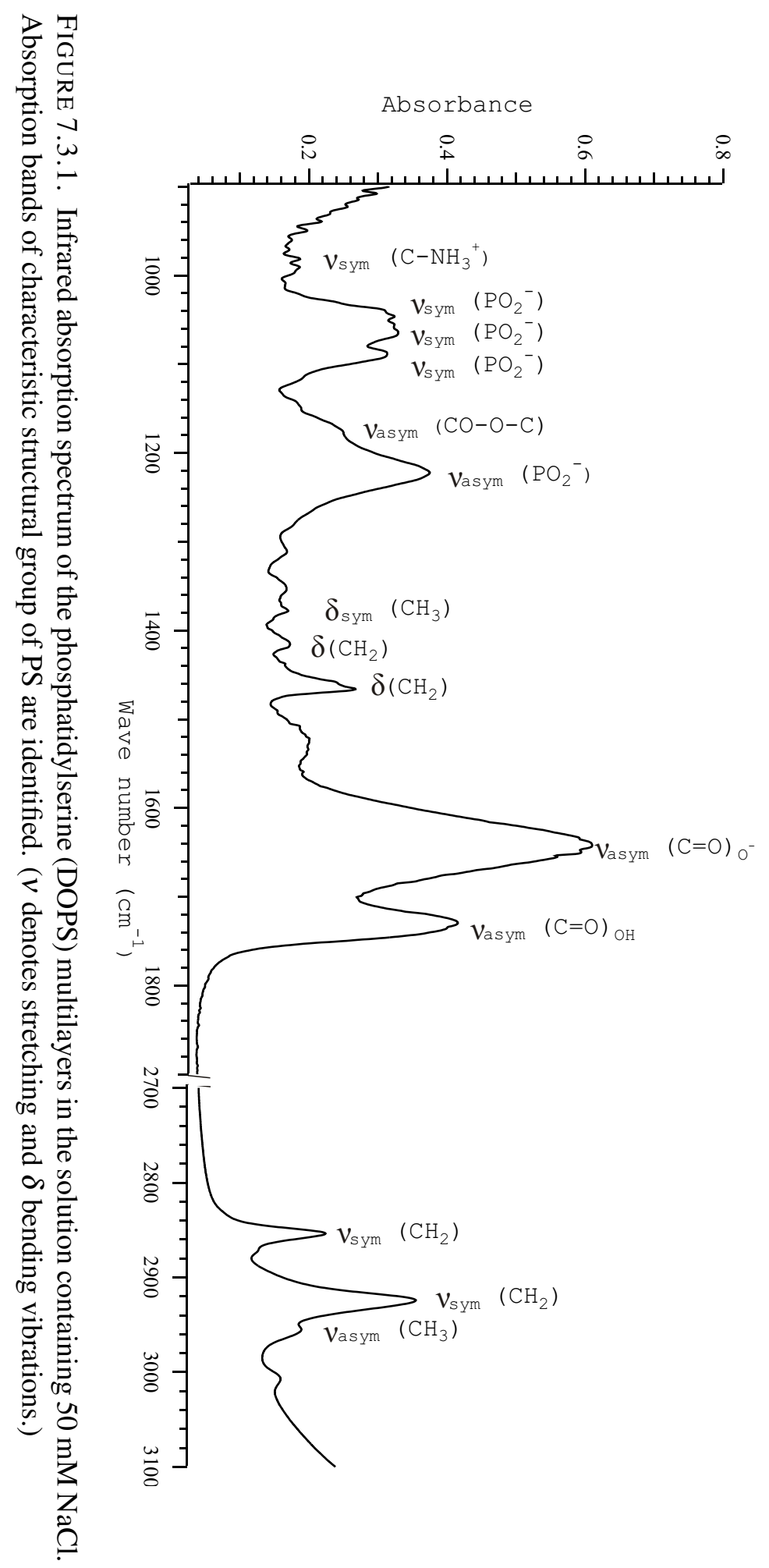


TABLE 1. Important infrared absorption bands of DOPS lipid. Assignments are based on [159, 161, 160].

\begin{tabular}{ccl}
\hline $\begin{array}{c}\text { Approximate frequency } \\
\left(\mathrm{cm}^{-1}\right)\end{array}$ & Assignment & \\
\hline 2957 & stretching & $v_{\text {asym }}\left(\mathrm{CH}_{3}\right)$ \\
2924 & stretching & $v_{\text {asym }}\left(\mathrm{CH}_{2}\right)$ \\
2853 & stretching & $v_{\text {sym }}\left(\mathrm{CH}_{2}\right)$ \\
1729 & stretching & $v_{\text {asym }}(\mathrm{C}=\mathrm{O})_{\mathrm{OH}}$ \\
1642 & stretching & $v_{\text {asym }}(\mathrm{C}=\mathrm{O})_{\mathrm{O}^{-}}$ \\
1466 & bending (scissoring) & $\delta\left(\mathrm{CH}_{2}\right)$ \\
1415 & bending (scissoring) & $\delta\left(\mathrm{CH}_{2}\right)$ \\
1377 & bending & $\delta_{\text {sym }}\left(\mathrm{CH}_{3}\right)$ \\
1222 & stretching & $v_{\text {asym }}\left(\mathrm{PO}_{2}^{-}\right)$ \\
1174 & stretching & $v_{\text {asym }}\left(\mathrm{CO}_{-} \mathrm{O}-\mathrm{C}\right)$ \\
1090 & stretching & $v_{\text {sym }}\left(\mathrm{PO}_{2}^{-}\right)$ \\
1063 & stretching & $v_{\text {sym }}\left(\mathrm{PO}_{2}^{-}\right)$ \\
1050 & stretching & $v_{\text {sym }}\left(\mathrm{PO}_{2}^{-}\right)$ \\
990 & stretching & $v_{\text {sym }}\left(\mathrm{NH}_{3}^{+}\right)$ \\
\hline
\end{tabular}

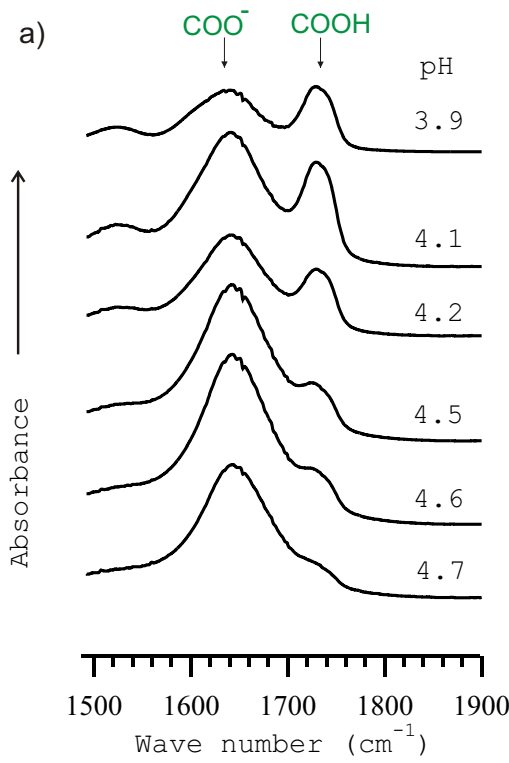

b)

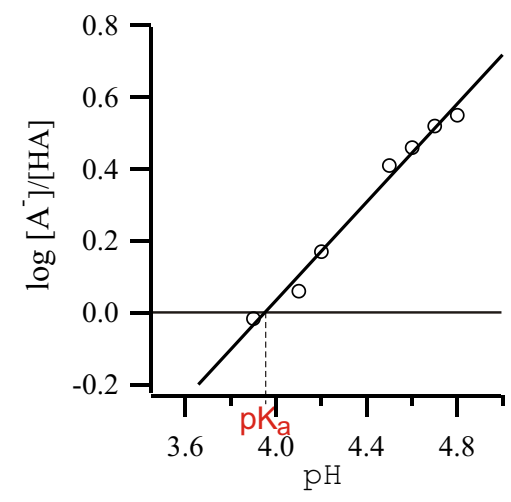

FIGURE 7.3.2. a) FT-IR spectra of the $1500-1900 \mathrm{~cm}^{-1}$ region of DOPS multilayers in the solution, containing $50 \mathrm{mM} \mathrm{NaCl}$, at different bulk $\mathrm{pH}$ values as indicated. b) Plot of $\log \left[\operatorname{int}\left(v(C=O)_{O^{-}}\right) / \operatorname{int}\left(v(C=O)_{O H}\right)\right]$ versus pH. Solid line represents the linear fit of the measured data.

Therefore from the plot of $\log \left[\right.$ intensity $\left(v(C=O)_{O^{-}}\right) /$intensity $\left.\left(v(C=O)_{O H}\right)\right]$ vs. pH (see Fig. 7.3.2 (b) ), the apparent $p K_{a}$ can be obtained.

The apparent $p K_{a}$ of the carboxyl group of hydrated DOPS was determined to be 3.97 \pm 0.20 . Gómez-Fernández and Villalaín [162] have obtained the apparent $p K_{a}=4.6$ for the carboxyl group of DPPS without the presence of salts by FT-IR method. 
The surface $\mathrm{pH}$ at which $50 \%$ of the group population is charged is defined as the intrinsic $p K$. The relation between the intrinsic and the apparent $p K_{a}$ is [20]:

$$
p K(\text { intrinsic })=p K_{a}(\text { apparent })+0.434 \frac{e \psi_{S}}{k_{B} T}
$$

where $e$ is the elementary charge, $\psi_{S}$ is the electrostatic surface potential, and $k_{B}$ and $T$ have their usual meanings. The surface potential $\psi_{S}$ of phosphatidylserine membrane was obtained from a relation between the surface charge density $\sigma$ and $\psi_{S}$, given by the Grahame equation ${ }^{1}$ for monovalent $1: 1$ electrolytes $[\mathbf{3 8}]$ :

$$
\sigma=\sqrt{8 \varepsilon_{w} \varepsilon_{0} k_{B} T[N a C l]} \sinh \left(e \psi_{S} / 2 k_{B} T\right)
$$

where $\sigma$ is in units of $\mathrm{Cm}^{-2}, \varepsilon_{w} \sim 80$ is water dielectric constant, $\varepsilon_{0}=8.854 \times 10^{-12} \mathrm{C}^{2} \mathrm{~J}^{-1} \mathrm{~m}^{-1}$ vacuum dielectric constant, $k_{B}=1.381 \times 10^{-23} \mathrm{JK}^{-1}$ Boltzmann's constant, $T$ absolute temperature, $\psi_{S}$ surface potential in $\mathrm{V}, e=-1.602 \times 10^{-19} \mathrm{C}$ electronic charge and $[\mathrm{NaCl}]$ is salt concentration in $\mathrm{M}\left(1 \mathrm{M}=1 \mathrm{moldm}^{-3}\right.$ and corresponds to a number density of $\rho=6.022 \times 10^{26} \mathrm{~m}^{-3}$ $[\mathbf{3 8}])$.

The headgroup of charged DOPS bilayer in the fluid state at $30^{\circ} \mathrm{C}$ with an area of $A=65.3 \AA^{2}$ [103] carries a single negative charge. This would correspond to the $\sigma=-0.23 \mathrm{Cm}^{-2}$. Then, the surface potential of phosphatidylserine membrane in an aqueous $50 \mathrm{mM} \mathrm{NaCl}$ solution, deduced from the Graham equation, is about $-147 \mathrm{mV}$. Finally, the intrinsic $p K$ for $-\mathrm{COOH}$ groups of DOPS in $50 \mathrm{mM} \mathrm{NaCl}$ solution at $300 \mathrm{~K}$ (DOPS is in fluid phase) is calculated according to Eq. 7.3 .2 to be $1.6 \pm 0.1$.

The intrinsic $p K$ value herein obtained under the present experimental conditions is at variance with those reported in the literature. Literature measurements for PS were carried out on black lipid membranes [164], dispersions [165], or monolayers [166]. The intrinsic $p K$ values for -COOH groups in PS were estimated by transmembrane potential measurements by the potentiometric technique to be 2.1 [164], by $\zeta$ potential measurements by electrophoresis to be $2.7[\mathbf{1 6 5}]$ and by measurement of the differential capacity of a lipid-coated electrode to be 3.3 [166]. This variability in estimated values could arise from the influence of physical factors such as ionic strength and temperature, which were not the same in the different estimations listed above. The length of the fatty acid chains, the type of phospholipid used, the size and organization of the mixtures and the different mixing ratios of fatty acid to phospholipid could also be sources of variability [167]. Vaz et al. [168] have evaluated the interfacial $\mathrm{pH}$ in lipid-water lamellar systems prepared from negatively charged lipids by use of a fluorescent $\mathrm{pH}$ indicator (chromophore attached to the polar head groups of lipids). At a fixed electrolyte concentration in the aqueous phase, they observed that the interfacial $\mathrm{pH}$ is 0.6 to $0.7 \mathrm{pH}$ units lower above the thermal phase transition of the lipid than

\footnotetext{
${ }^{1}$ In the Gouy-Chapman model (see Chapter 2 a uniform charge density on a planar interface and counterions as point charges are assumed. Neglecting structural changes of the membrane which might be induced via the ionic environment, the Grahame equation is obtained [163].
} 
it is below this temperature. This could explain relatively low value of the intrinsic $p K$ estimated in our experiment.

\subsection{Supported DOPC:DOPC(4:1) in fluid phase interacting with gold particles}

Supported DOPC/DOPS (4:1) bilayers were prepared on silicon support as described in Chapter 3 Experiments described below were performed at neutral $\mathrm{pH} 7.4$ and at acidic $\mathrm{pH}$ 4.6. The weak polyelectrolyte $\mathrm{PAH}$ is not fully protonated at neutral $\mathrm{pH}$. The degree of protonation of PAH at neutral $\mathrm{pH}$ was estimated from potentiometric titration to be $\sim 80 \%[\mathbf{1 6 9}, \mathbf{1 7 0}]$. At pH 4.6, PAH is fully dissociated $[\mathbf{1 6 9}, 1 \mathbf{1 7 0}]$.

The surface potential $\zeta$ of PAH-coated AuNPs was measured by the producer to be $48.5 \mathrm{mV}$ at neutral $\mathrm{pH}[\mathbf{1 5 6}]$. We estimated the surface potential of about $60 \mathrm{mV}$ for PAH-coated AuNPs at $\mathrm{pH} 4.6$.

The phosphatidylserine membrane is fully ionized at neutral $\mathrm{pH}$ and the degree of ionization at $\mathrm{pH} 4.6$ is calculated to be 95 to $100 \%$, according:

$$
\text { \%ionization }=\frac{100}{1+10^{(p K-p H)}}
$$

where the intrinsic dissociation constant $p K$ ranges from 3.3 to 1.6. Later we will assume that the PS membrane is fully ionized at $\mathrm{pH}$ 4.6.

Experimental pH 4.6 was attained with $10 \mathrm{mM}$ acetic acid buffer and $\mathrm{pH} 7.4$ with $10 \mathrm{mM}$ HEPES/NaOH buffer. For the experiments performed at different ionic straights, $0 \mathrm{mM} \mathrm{NaCl}, 50$ $\mathrm{mM} \mathrm{NaCl}, 100 \mathrm{mM} \mathrm{NaCl}$ were added respectively. SUVs were prepared in respective buffers, as described in Chapter 3 , and fused onto a clean $\mathrm{Si}$ wafer (for X-ray reflectivity measurement) or a clean Si foil (for fluorescence microscopy). PAH-coated gold nanoparticles (size of $64 \pm$ $5 \mathrm{~nm})$ were added so that their final concentration was $0.33 \mu \mathrm{M}\left(\sim 4.5 \times 10^{7}\right.$ particles $/ 2 \mathrm{ml} \mathrm{solu}$ tion), or $3.3 \mathrm{nM}\left(\sim 4.5 \times 10^{5}\right.$ particles $/ 2 \mathrm{ml}$ solution). After $30 \mathrm{~min}$ incubation time, unattached nanoparticles were gently washed and samples were observed by fluorescence microscopy and $\mathrm{X}$-ray reflectivity methods.

Depending on the experimental conditions (different $\mathrm{pH}$, buffer ionic strength) different responses of the bilayer to the AuNPs were observed: they range from no change to the bilayer to complete disruption and removal of the bilayer from the surface. Fig. 7.4.1 shows gray scale fluorescence images of supported DOPC/DOPS (4:1) bilayers before and after injecting $0.3 \mu \mathrm{M}$ of PAH-coated AuNPs.

At $\mathrm{pH} 7.4$, PS is fully dissociated and particles are $\sim 80 \%$ charged. In the $10 \mathrm{mM} \mathrm{NaCl}$ buffer solution (see Fig. 7.4.1 (b)) we observed, that in several places the silicon substrate can be seen through defects, holes $(\sim 1 \mu \mathrm{m}$ in diameter) in the bilayer. Membrane seems to be laterally disturbed. Areas with apparently higher fluorescence intensity could be particles partially coated by the membrane. We did not observe floating lipid aggregates.

At pH 4.6, PS and particles are assumed to be fully charged. In fluorescence images shown in Fig. 7.4.1 (c) we observed that the bilayer exhibits defects (in a form of holes, floating and attached vesicles, or aggregates), or is damaged. Extended parts of the membrane are removed from the substrate. Aggregated lipid-particle complexes with high fluorescence intensity and lipid vesicles, 

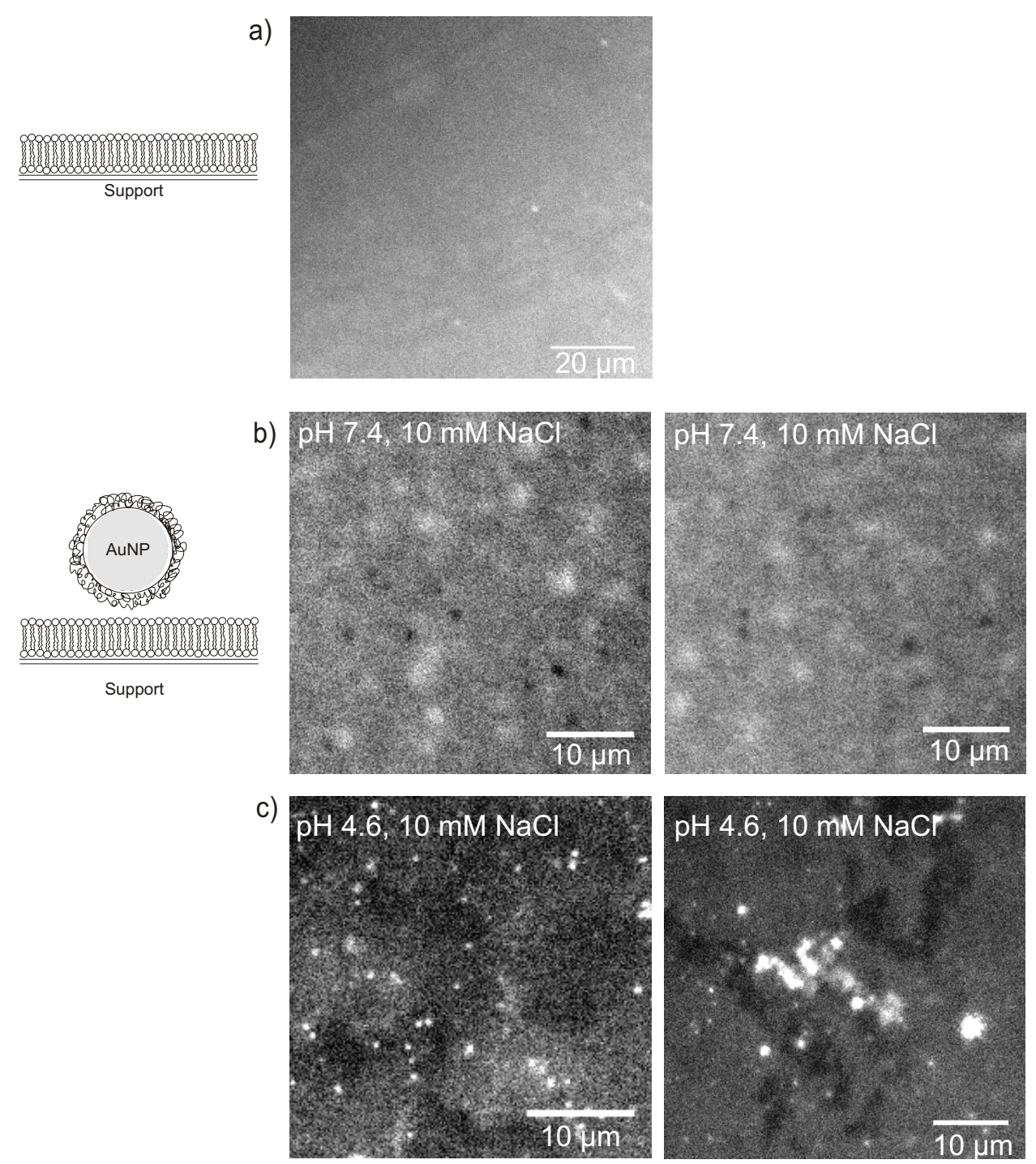

FIGURE 7.4.1. Gray-scale fluorescence images of DOPC:DOPS (4:1) supported lipid bilayers containing $0.1 \%$ Texas Red (a) before adding PAH-coated AuNPs; (b) after adding of $0.33 \mu \mathrm{M}$ PAH-coated AuNPs in $10 \mathrm{mM} \mathrm{NaCl}$ buffer solution, pH 7.4 and (c) after adding of $0.33 \mu \mathrm{M}$ PAH-coated AuNPs in $10 \mathrm{mM} \mathrm{NaCl}$ buffer solution, $\mathrm{pH} 4$.6. Radius of particle is $\sim 34 \mathrm{~nm}$, thickness of lipid bilayer is $\sim 4$ $\mathrm{nm}$. In several places the silicon substrate (dark) can be seen through defects in the bilayer (lighter colored areas) caused by AuNPs.

probably filled with particles, were floating in the solution. This indicates strong interaction of membrane with particles. The removal of the SPB from the surface was not complete: some of the lipids remained at the surface after disruption.

$\mathrm{X}$-ray reflectivity measurements were carried out on an in-house D8 setup as described in Chapter 5. Reflectivity curves from single lipid membranes at pH 7.4 and 4.6 respectively, were measured before and after adding of particles (see Fig. 7.4.2). The particles diffused towards the substrate within a few minutes. Fig. 7.4.2 (a) shows first two minima of the reflectivity curves (see an example of complete DOPC/DOPS (4:1) reflectivity curve in Fig. 1 in Chapter 5) of a 

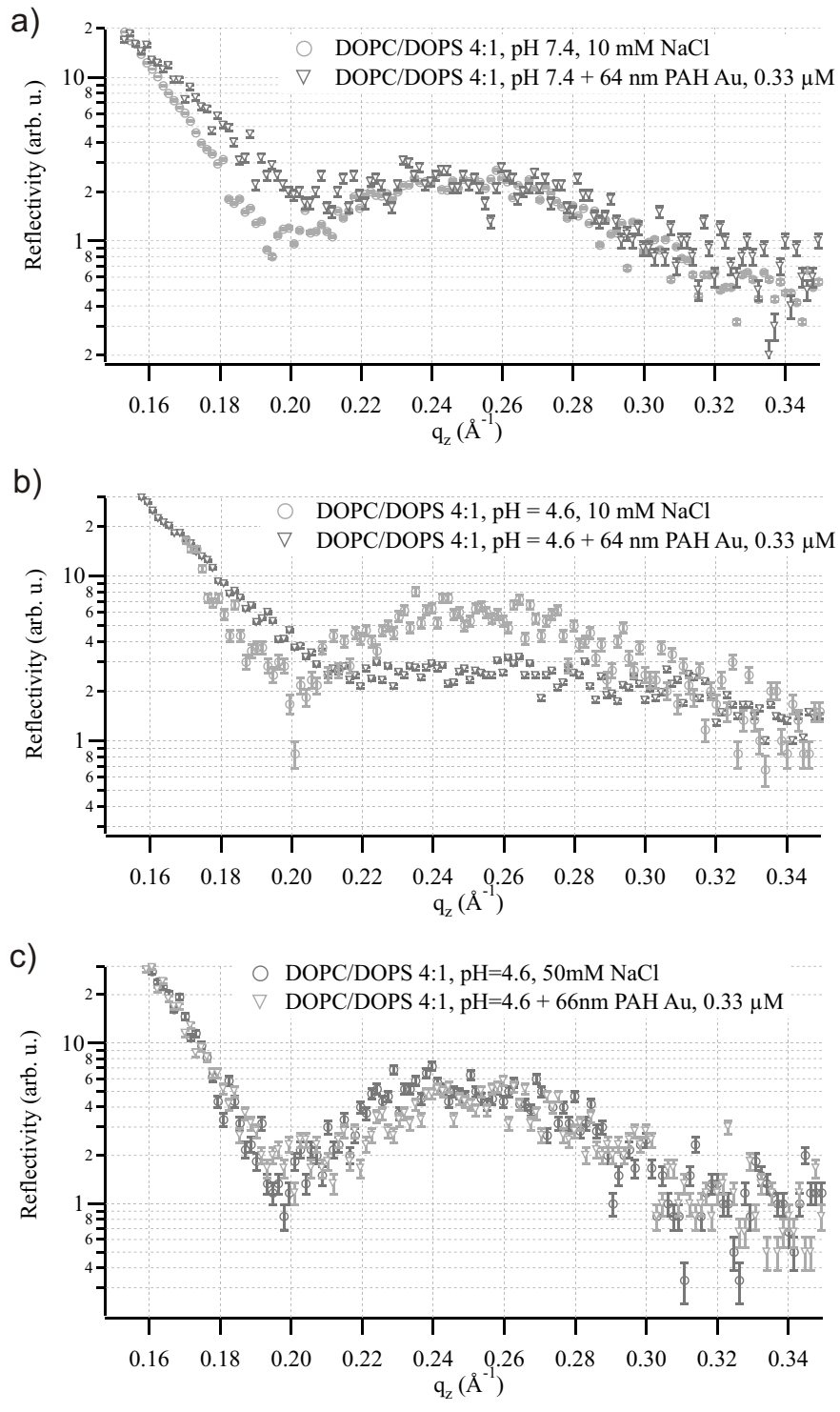

FIGURE 7.4.2. X-ray reflectivity curves in the $\mathrm{q}_{z}$ range of $0.15-0.34$ of DOPC:DOPC (4:1) supported lipid bilayers before and after adding PAH-coated AuNPs (a) in the $10 \mathrm{mM} \mathrm{NaCl}$ buffer solution, $\mathrm{pH} \mathrm{7.4;} \mathrm{(b)} \mathrm{in} \mathrm{the} 10 \mathrm{mM} \mathrm{NaCl}$ buffer solution, $\mathrm{pH} 4.6$ and (c) in the $50 \mathrm{mM} \mathrm{NaCl}$ buffer solution, $\mathrm{pH} 4.6$.

supported DOPC/DOPS (4:1) bilayer at $\mathrm{pH} 7.4$ and in low ionic strength buffer before and after injecting $0.33 \mu \mathrm{M}$ of PAH-coated AuNPs. Fig. 7.4.2 (b, c) shows the reflectivity curves at $\mathrm{pH} 4.6$ in $10 \mathrm{mM} \mathrm{NaCl}$ and $50 \mathrm{mM} \mathrm{NaCl}$ acetic acid buffer, respectively, before and after injecting PAH AuNPs.

X-ray reflectivity was used to probe change in thickness, or coverage of a single lipid bilayer after interaction with AuNPs in comparison with the typical reflectivity profile from a bare single lipid bilayer. Reflectivity was measured in the $\mathrm{q}_{z}$ range of $0.15-0.34$. Without further fitting procedure, we may estimate information as: a relative bilayer thickness changes from the shift of the minima; increase of a reflected intensity might indicate an increased electron density contrast 
between a probe and a buffer; decreasing of a reflected intensity might be caused by decreasing of a membrane surface coverage. Disappearing of minima indicates membrane damage.

Change in a surface coverage, or a thickness of the bilayer is observed in the case of membraneparticles interaction in the low ionic strength solution at $\mathrm{pH}$ 7.4.

In the case of interactions in the low ionic strength solution ( $\mathrm{pH} 4.6)$, when particles are fully charged, disappearing of typical reflectivity signal from a supported membrane was observed. From this we might conclude that the membrane coverage decreases, what indicates the membrane damage. This observation is consistent with fluorescence results.

When AuNPs were added to supported bilayers in a higher ionic strength buffer $(50 \mathrm{mM} \mathrm{NaCl}$, $\mathrm{pH}=4.6$ ), the SPB was observed to remain intact (see Fig. 7.4.2 (c)). Attachment of nanoparticles might be concluded from increased reflected intensity.

\subsection{Supported DPPC:DPPC(4:1) in gel phase interacting with gold particles}

DPPC:DPPS (4:1) supported lipid bilayers were formed above the transition temperature of used lipids $\left(\sim 45^{\circ} \mathrm{C}\right)$. Subsequently, samples were cooled down to room temperature and AuNPs were added. X-ray reflectivity curves at two different $\mathrm{pH}$ (pH 7.4 and 4.6) and low ionic strength $(10 \mathrm{mM} \mathrm{NaCl})$ conditions are shown in Fig. 7.5.1

In the gel phase at $\mathrm{pH} 7.4$ we did not observe a significant change in reflectivity comparing to the bare membrane. At $\mathrm{pH} 4.6$, subsequent increase of concentration of added particles (from 3.3 $\mathrm{nM}$ to $0.33 \mu \mathrm{M}$ ) corresponds to increasing in reflected intensity from lipid bilayer-AuNPs sample.

\subsection{DOPC:DOPC(4:1) GUV interacting with gold particles}

Because membrane curvature is constrained in supported lipid bilayers, we used as well GUVs as a model system for lipid membranes. Preparation of GUVs is described in Chapter 3 . Solution of GUVs was mixed with PAH-coated gold nanoparticles of diameter $64 \pm 5 \mathrm{~nm}$ so that their final concentration was $0.33 \mu \mathrm{M}\left(\sim 4.5 \times 10^{5}\right.$ particles/20 $\mu 1$ solution). Time sequence series $(3 \mathrm{~min})$ of phase contrast images of giant lipid vesicles after adding of PAH-coated AuNPs at pH 7.4 were taken (see Figs. 7.6.1, 7.6.2). We observed morphological changes of giant unilamellar vesicle upon adhesion of nanoparticles. First, tiny tubes started to grow from the vesicle, followed by growing of larger tubes and budding of vesicle.

These interesting phenomena were already described by Noguchi et al. [71]. They have studied the adhesion of a nanoparticle from the outside of a vesicle by Monte Carlo simulation method and have observed that the nanoparticle started to bud to the inside of the vesicle first. The membrane then encapsulated the nanoparticle. To reduce the connection region, the encapsulated nanoparticle gradually moved to the outside of the vesicle and finally, the vesicle changes to the stalk state.

Experiments on giant phase separated vesicles have demonstrated that phase separation can drive membrane curvature. Different curvature between the phases caused one phase "bulging" from the other [171]. According to this study, two distinct physical characteristics contribute to morphology: differences in bending rigidity between the phases and line tension associated with the phase boundary. The importance of rigidity (bending modulus) in determining response to 

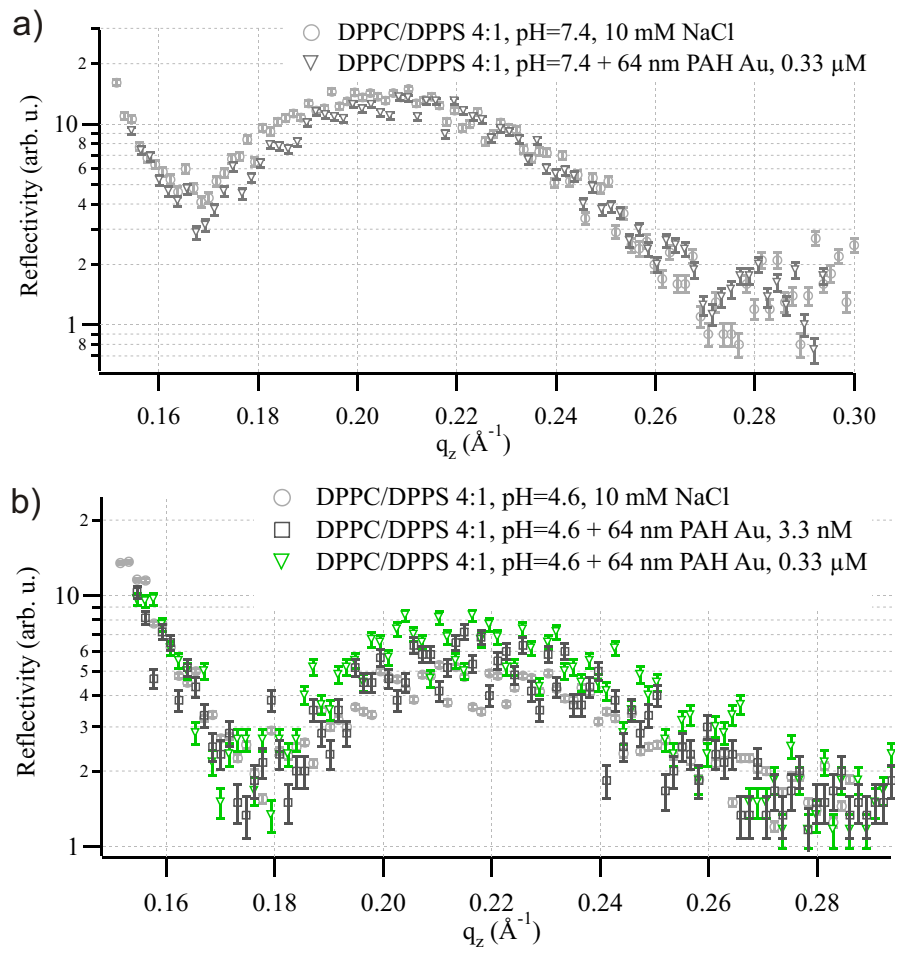

FIGURE 7.5.1. X-ray reflectivity curves in the $\mathrm{q}_{z}$ range of $0.15-0.30$ of DPPC:DPPC (4:1) supported lipid bilayers before and after adding PAH-coated gold AuNPs (a) in the $10 \mathrm{mM} \mathrm{NaCl}$ buffer solution, $\mathrm{pH} 7.4$; (b) in the $10 \mathrm{mM}$ $\mathrm{NaCl}$ buffer solution, $\mathrm{pH}$ 4.6. Supported lipid bilayers were formed above the transition temperature of used lipids $\left(\sim 45^{\circ} \mathrm{C}\right)$. Then, the samples were cooled down to room temperature and AuNPs were added.

curvature is clear: more rigid membranes require more energy to bend. Line tension generates curvature independent of rigidity. The energy associated with a phase boundary is proportional to the product of the line tension and the length of the boundary. A two-dimensional phase separated membrane can therefore lower this energy by causing one phase to bulge, keeping the area of the phases fixed, but lowering the length of the phase boundary [172]. Here, we can speculate, that one possible reason for budding events is demixing of lipids and formation of domains rich in PS, caused by particles attachment. Jülicher and Lipowski [154] have shown that vesicles composed of two fluid domains undergo domain-induced budding. These budded states should be observable by optical microscopy.

Under the same experimental conditions ( $\mathrm{pH} 7.4,10 \mathrm{mM} \mathrm{NaCl}$ ), we observed hole formation in supported lipid membranes was observed. Rossetti et al. [173] have investigated interactions between the graft copolymer poly(L-lysine)-g-poly(ethylene glycol) and solid-supported lipid system by a combination of microscopic and spectroscopic techniques. Their experiments indicate that defects in SPBs play a role in disruption process. Formation of holes in a lipid bilayer might be caused by the fact that particles adsorb stronger to the silicon than to DOPC/DOPS membrane and thereby displace the lipid molecules around them. Some authors have argued that preparing a defect-free SPB by a vesicle fusion procedure is virtually impossible [54, 87, 174]. 

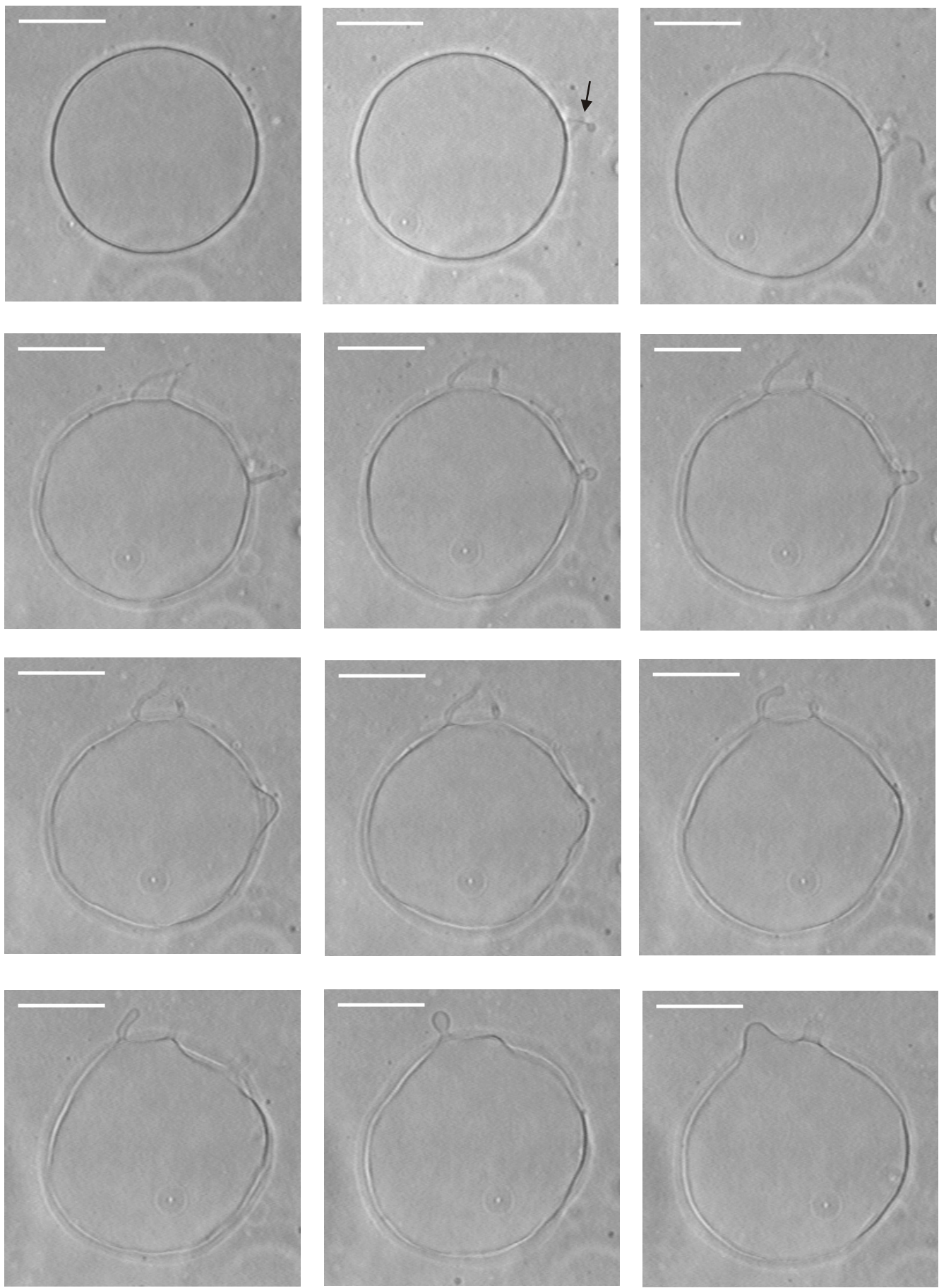

FIGURE 7.6.1. First part of time sequence ( $3 \mathrm{~min}$ ) of phase contrast images of giant DOPC/DOPS (4:1) vesicles mixed with PAH-coated AuNPs at $0.33 \mu \mathrm{M}$ in the $10 \mathrm{mM}$ buffer solution, $\mathrm{pH}$ 7.4. We observed the morphological changes of lipid vesicle, as budding events and a grow of tubes. Bar, $10 \mu \mathrm{m}$. Objective, 40x. 

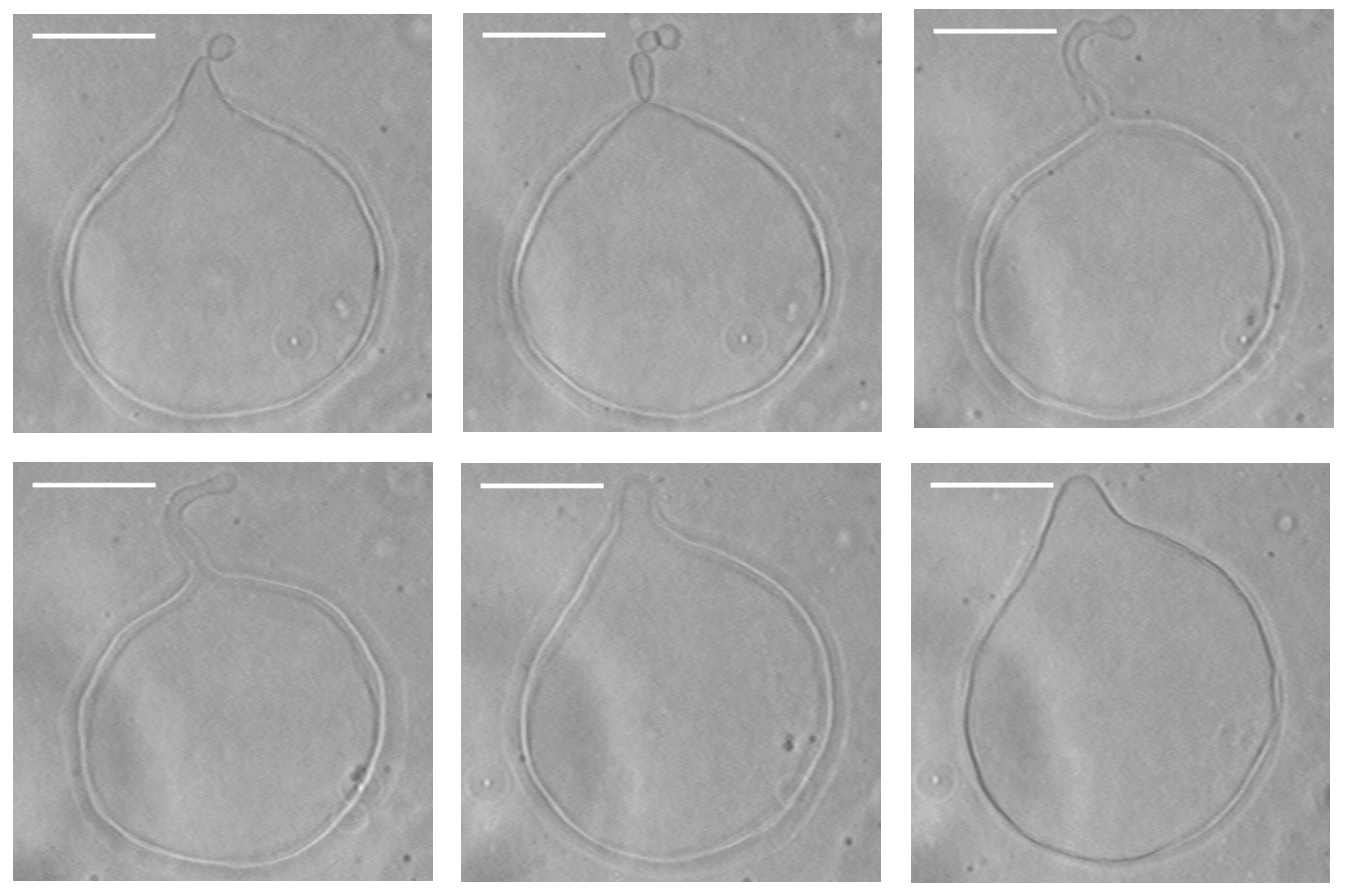

FIGURE 7.6.2. Second part of time sequence (3 min) of phase contrast images of giant DOPC/DOPS (4:1) vesicles mixed with PAH-coated AuNPs at $0.33 \mu \mathrm{M}$ in the $10 \mathrm{mM}$ buffer solution, $\mathrm{pH}=7.4$. We observed morphological changes of giant lipid vesicle, as budding events and a grow of tubes. Bar, $10 \mu \mathrm{m}$. Objective, 40x.

Time sequence series ( $2 \mathrm{~min} 44 \mathrm{sec}$ ) of phase contrast images of giant lipid vesicles after adding $0.3 \mu \mathrm{M}$ of particles at $\mathrm{pH} 4.6$ are shown in Fig. 7.6.3. We observed that attachment of particles to the membrane terminated in membrane rupture (probably a critical membrane tension was overcome by particle attachment). Strong electrostatic interactions between fully charged AuNPs and the $20 \%$ anionic lipid membrane could induce latteral reorganization of lipids and their diffusion toward particle absorption sites. Yaroslavov et al. [175] have shown that the adsorption of a polyelectrolyte to oppositely charged vesicles induces flip-flop in the membrane. Extensive lateral diffusion and flip-flop events might destabilize the membrane as well.

\subsection{Electrostatic interactions between membrane and gold particles}

The electrostatic interaction energy between a charged PAH-coated gold nanoparticle at a separation of $D$ from a membrane surface is calculated in the Derjaguin and superposition approximation [176] as:

$$
E_{e l}=4 \pi \varepsilon \varepsilon_{0} R \psi_{\text {membrane }}^{\text {eff }} \psi_{\text {particle }}^{\text {eff }} \exp (-\kappa D),
$$

where $\varepsilon \varepsilon_{0}$ is the total electric permittivity, $\kappa^{-1}$ Debye screening length, $R$ the particle radius, and $D$ is the distance between the membrane and the particle. 

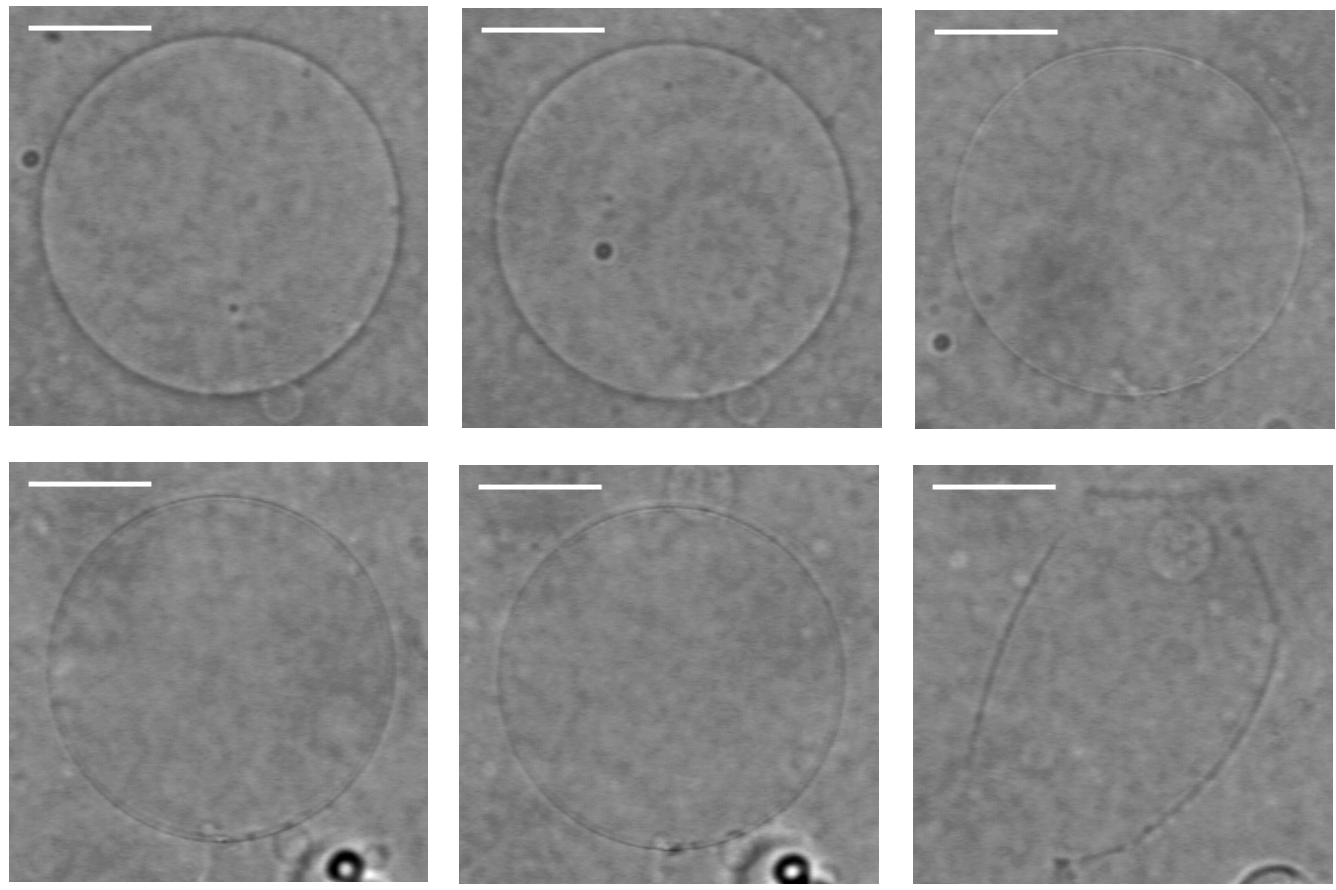

FIGURE 7.6.3. Time sequence ( $2 \mathrm{~min} 44 \mathrm{sec}$ ) of phase contrast images of giant DOPC:DOPS (4:1) vesicles mixed with PAH-coated AuNPs at $0.33 \mu \mathrm{M}$ in the $10 \mathrm{mM}$ buffer solution, $\mathrm{pH}$ 4.6. We observed disruption of vesicle. Bar, $10 \mu \mathrm{m}$. Objective, 40x.

$\psi_{\text {membrane,particle }}^{\text {eff }}=\left(4 k_{B} T / e\right) \tanh \left(e \psi_{\text {membrane, particle }} / 4 k_{B} T\right)$ denotes the effective electrostatic potential of either the membrane or the particle surface ( $e$ is discrete anionic or protonic charge $)^{2}$, where $\psi_{\text {membrane,particle }}$ is a surface potential of either the membrane or the particle surface.

The Debye length falls with increasing ionic strength and valence of the ions in the solution. For aqueous electrolyte (salt) solution at $298 \mathrm{~K}$ the Debye length (in $\mathrm{nm}$ ) is

$$
1 / \kappa=0.304 /[\mathrm{NaCl}]^{\frac{1}{2}}
$$

for 1:1 electrolytes such as $\mathrm{NaCl}[\mathbf{3 8}]$, where the concentration unit $[\mathrm{NaCl}]$ is in moles/liter $(\mathrm{M})$. For example, for $\mathrm{NaCl}$ solutions, $1 / \kappa \approx 0.96 \mathrm{~nm}$ at $100 \mathrm{mM}, \approx 1.35 \mathrm{~nm}$ at $50 \mathrm{mM}$ and $\approx 3.04 \mathrm{~nm}$ at $10 \mathrm{mM}$.

The surface charge density $\sigma$ of the membrane surface composed of acidic and neutral lipids of the headgroup area $A=71 \AA^{2}[\overline{60}]$ is $\sigma=-0.22 \mathrm{Cm}^{-2}$. The electric surface potential of the DOPC:DOPS (4:1) lipid mixture $\left(\sigma=-0.044 \mathrm{Cm}^{-2}\right)$, calculated using Eq 7.3.3, for different $\mathrm{pH}$ and salt concentrations are listed in Tab. 2 .

Finally, electrostatic interaction energies calculated using Eq7.7.1 for different $\mathrm{pH}$ and salt concentrations, at a separation of $\mathrm{D}=0.4 \mathrm{~nm}$ are listed in Tab. 2. $\mathrm{D}=0.4 \mathrm{~nm}$ allows for one layer of strongly bound water molecules (spherical solvent molecules of diameter $\sim 0.25 \mathrm{~nm}[\mathbf{3 8}]$ ) on each surface - for hydrophilic PAH-coated AuNPs and hydrophilic membrane surface.

\footnotetext{
${ }^{2}$ Electronic charge $e=-1.60 \times 10^{-19} \mathrm{C}$ by definition. Permittivity of free space $\varepsilon_{0}=8.854 \times 10^{-12} \mathrm{C}^{2} \mathrm{~J}^{-1} \mathrm{~m}^{-1}$. Dielectric constant of water $\varepsilon_{\text {water }}=80.1$ at $293 \mathrm{~K}$ by definition. $1 k_{B} T=4.114 \times 10^{-21} \mathrm{~J}$ at $298 \mathrm{~K}$.
} 
TABLE 2. Surface potentials of the PC/PS 4:1 membrane at different ionic strength conditions and electrostatic interaction energies $\left(E_{e l} / k_{B} T\right)$ with PAH-coated AuNPs. Radius of the particle, including polyelectrolyte layer, is $\sim 34 \mathrm{~nm}$.

\begin{tabular}{cccccc}
\hline $\mathrm{pH}$ & {$[\mathrm{NaCl}] / \mathrm{mM}$} & $1 / \kappa / \mathrm{nm}$ & $\psi_{\text {membrane }} / \mathrm{mV}$ & $\psi_{\text {particle }} / \mathrm{mV}$ & $\mathrm{E}_{e l} / k_{B} T$ \\
\hline 7.4 & 10 & 3.04 & -106.8 & 48.5 & -233.8 \\
4.6 & 10 & 3.04 & -106.8 & 60 & -281.7 \\
7.4 & 50 & 1.35 & -68.4 & 48.5 & -148.2 \\
4.6 & 50 & 1.35 & -68.4 & 60 & -178.6 \\
7.4 & 100 & 0.96 & -53.6 & 48.5 & -108.2 \\
4.6 & 100 & 0.96 & -53.6 & 60 & -130.2 \\
\hline
\end{tabular}

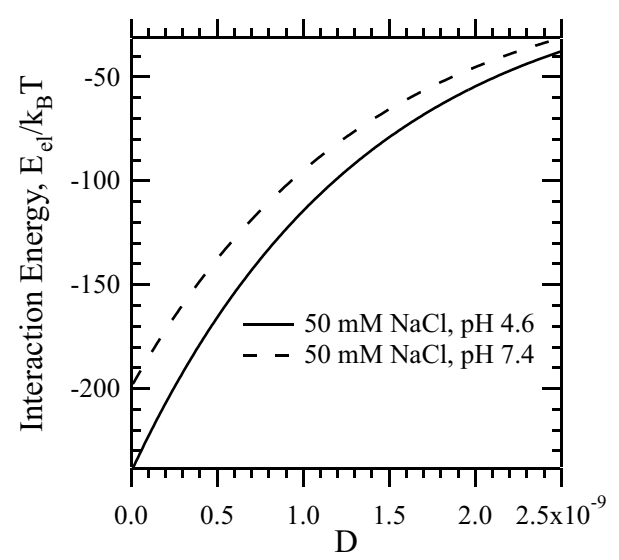

FIGURE 7.7.1. Interaction energies between PC/PS (4:1) membrane and PAHcoated gold nanoparticle (radius $\sim 34 \mathrm{~nm}$ ) plotted as a function of separation $D$, for $\mathrm{pH} 7.4$ and $\mathrm{pH} 4.6$.

Electrostatic interaction energies between PC/PS (4:1) membrane and PAH-coated gold nanoparticle plotted as a function of separation $D$, for $\mathrm{pH} 7.4$ and $\mathrm{pH} 4.6(50 \mathrm{mM} \mathrm{NaCl})$ are shown in Fig. 7.7.1.

Wrapping of a colloids by a membrane: The elastic membrane behavior is described by the local bending energy per area, $E_{\text {bend }}=\frac{1}{2} k\left(c_{1}+c_{2}\right)^{2}$, where $c_{1}$ and $c_{2}$ are local principal curvatures of the two-dimensional membrane surface and $k$ is a bending modulus (with units of energy) [39]. The particle provides a new source of adhesion energy for the membrane. Adhesion is driven by a contact energy per unit area $w$ and is opposed by the requirement to bend the membrane, as well as the work of pulling excess membrane towards the wrapping site against a prescribed lateral tension $\sigma$ [177]. The adhesion energy equals $-2 \pi R^{2} z w$, while bending and tension contributions are found to be $4 \pi z k$ and $\pi R^{2} z^{2} \sigma$, respectively. $z$ denotes the degree of wrapping $(z=1-\cos \alpha$ ) [177]. From tension and bending modulus one can construct a length $\lambda$, specific to the membrane, according to:

$$
\lambda=\sqrt{\frac{k}{\sigma}}
$$

Membrane deformations on a length scale smaller than $\lambda$ are mainly controlled by the bending energy, while the tension is predominant on larger scales. 
TABLE 3. Ratios of adhesion and critical adhesion energy $\left(w / w_{c}\right)$ for the PC/PS 4:1 membrane and PAH-coated AuNPs in different salt concentration and $\mathrm{pH}$ buffers.

\begin{tabular}{cccc}
\hline $\mathrm{pH}$ & {$[\mathrm{NaCl}] / \mathrm{mM}$} & $\left(\mathrm{gel}, k=250 k_{B} T\right) w_{a} / w_{c}$ & $\left(\right.$ fluid,$\left.k=21 k_{B} T\right) w_{a} / w_{c}$ \\
\hline 7.4 & 10 & 0.15 & 1.7 \\
4.6 & 10 & 0.18 & 2.1 \\
7.4 & 50 & 0.1 & 1.1 \\
4.6 & 50 & 0.12 & 1.4 \\
7.4 & 100 & 0.08 & 0.8 \\
4.6 & 100 & 0.09 & 0.9 \\
\hline
\end{tabular}

Bending modulus of the DPPC bilayer at $300 \mathrm{~K}$ (gel phase) has been measured by Lee et al. to be $\sim 250 k_{B} T[\mathbf{1 7 8}]$. For unsaturated DOPC bilayers in the liquid phase $k$ is typically $\sim 21 k_{B} T$ [103, 179].

Tensions of cellular membranes reported in literature vary between $0.003 \mathrm{dyne} / \mathrm{cm}$ and 1 dyne/cm [180]. Giant vesicles with diameters above a few microns, can be prepared with low membrane tension. The tension of GUVs is typically about $\sigma \sim 2 \times 10^{-5} \mathrm{dyne} / \mathrm{cm}[\mathbf{1 8 1}]$. A planar bilayer has a tension on the order of several dyne/cm [182]. In this study, we will consider the surface tension $\sim 2$ dyne/cm for planar lipid membranes [183].

For instance, with a GUV's tension $\sigma \sim 2 \times 10^{-5}$ dyne/cm and a bending modulus $\sim 21 k_{B} T$, we obtain $\lambda \simeq 2 \mu \mathrm{m}$. In this case, $R \ll \lambda$ (radius of the particle is $\sim 34 \mathrm{~nm}$ ), we will restrict ourselves to the bending-dominated low-tension regime $(\sigma \rightarrow 0)$.

For $\sigma=0$ colloids do not adhere at low adhesion energy $w<w_{c}=2 k / R^{2}$, whereas full wrapping occurs above $w_{c}$ with no energy barrier to be overcome [69]. Then, critical adhesion energy, $w_{c}=2 k / R^{2}$, is defined as the energy per unit area required to bend a bilayer with zero spontaneous curvature to a spherical cup.

Ratios of adhesion and critical adhesion energy $\left(w_{a} / w_{c}\right)$ for the PC/PS 4:1 membrane, both in gel and fluid phases, and PAH-coated AuNPs in different salt concentrations and $\mathrm{pH}$ buffers are listed in Tab. 3. For the gel phase membrane, we estimated $w / w_{c}<0.18$ in all experimental conditions. That means, that the adhesion energy is not high enough to cause bending of the gel phase bilayer. This is in agreement with our experimental observations.

For the fluid phase PC/PS 4:1 bilayer we estimated full wrapping $\left(w / w_{c}>1\right)$ of the particle by the GUV's bilayer in buffer solutions containing $10 \mathrm{mM} \mathrm{NaCl}$ and $50 \mathrm{mM} \mathrm{NaCl}$, with both $\mathrm{pH}$ 7.4 and 4.6. We predict that $100 \mathrm{mM} \mathrm{NaCl}$ might be sufficient to screen the electrostatic vesicle membrane-colloid attraction to avoid wrapping of a particle by a vesicle membrane.

In our experiments with supported lipid membranes we observed that already $50 \mathrm{mM} \mathrm{NaCl}$ was sufficient to avoid full wrapping of colloids by the membrane, followed by the membrane damage. From experimental results under the $10 \mathrm{mM} \mathrm{NaCl}, \mathrm{pH} 7.4$ condition we deduce only a partial wrapping of colloids by a membrane. 


\subsection{Conclusion}

The results regarding the interactions between polyelectrolyte coated AuNPs and both supported lipid membranes and giant unilamellar vesicles demonstrate the importance of the $\mathrm{pH}$ and ionic strength as a modulating parameter of the interaction. We have shown that depending on the experimental conditions (different $\mathrm{pH}$, buffer ionic strength) different responses of the bilayer to the PAH-coated AuNPs were observed: they range from no change to the bilayer to complete disruption and removal of the bilayer from the surface. The salt concentration, $\mathrm{pH}$, phase state and lateral tension of the membrane was found to play a significant role in the interaction of the bilayer with particles. PAH-coated AuNPs disrupt fluid supported phospholipid bilayers in a low ionic strength buffer $(10 \mathrm{mM} \mathrm{NaCl})$, but not in a high ionic strength one $(50 \mathrm{mM} \mathrm{NaCl}) . \mathrm{Gel}$ supported bilayers were observed to remain intact. We have shown that AuNPs induce structural changes of vesicles: budding and tube formation at neutral $\mathrm{pH}$, but vesicles were disrupted at acidic $\mathrm{pH}$, when particles are fully dissociated.

Polyelectrolytes were mostly used a decade ago as a protective layer against coagulation and adsorption. Other important polyelectrolyte-specific application in solution include the control of visco-elastic properties and responsiveness to small variations of solvent conditions [184]. These properties are now promising tools to tune and control lipid membranes and to trigger biomimetic responses, including budding, or formation of lateral domains. Critical sensitivity of weak polyelectrolyte chains to solvent quality, $\mathrm{pH}$, or temperature has opened new ways to specifically destabilize and break membranes, artificial ones as well as living cells. Nevertheless, the lateral pressure developed by polyelectrolyte charged hydrophilic chains can markedly modify the bending and rigidity.

From a practical point of view, the response to $\mathrm{pH}$ of polyelectrolyte coated gold nanoparticles suggest new opportunities to develop sensitive sensors or triggered release of probe particles in biological media. 



\section{CHAPTER 8}

\section{Summary}

The aim of this thesis was to study the structure of charged lipid membranes and their interaction with oppositely charged colloidal particles. Adhesion of colloidal particles onto oppositely charged, mixed lipid membranes is a ubiquitous phenomenon encountered in biotechnology, drug delivery, and cellular biology.

In the first part, we focused on the preparation of supported one and two-component single lipid bilayers with positive, neutral and negative charge. X-ray reflectivity was used to investigate detailed structural characteristics of these mixed bilayers, as well as the effect of the bilayer charge on the water spacing between bilayer and silicon substrate. Structural parameters, such as membrane thickness, area per lipid, water thickness, and the number of water molecules per lipid were calculated directly from an electron density profile of the model bilayers. A splitting of the density profile in the headgroup region of mixed bilayers was observed, which we associate with calcium induced lateral demixing of the two lipid components. Furthermore, we have shown that such analysis does not rely only on synchrotron radiation, but can also be carried out at sealed tube instruments with optimized equipment.

In the second part, we used scanning transmission X-ray microscopy to image positively charged polystyrene latex microspheres which bind onto previously characterized supported twocomponent lipid membranes, composed of charged and neutral lipid species. Data have been collected on dried colloids as well as wet lipid membrane/polystyrene microspheres. The amount of the carbon was determined quantitatively from the difference in the X-ray absorption. Preferential binding of microspheres to the oppositely charged bilayer was observed. Surprisingly, the bound microspheres aggregated on the lipid bilayer patch, despite equal charges and inter-particle repulsion. The feasibility of lipid thin films carbon $K$-edge NEXAFS experiments with X-ray radiation generated from compact sources based on a laser-produced plasma was successfully demonstrated and we presented a small data bank of the NEXAFS spectra of some of the most common lipids.

The aim of the third part was to study how charged membranes response to oppositely charged polyelectrolyte-coated gold nanoparticles. We have used fluorescence microscopy and X-ray reflectivity methods to probe the interactions between polyelectrolyte coated gold nanoparticles and supported lipid membranes as well as giant unilamellar vesicles. The results demonstrate the importance of the $\mathrm{pH}$ and ionic strength as a modulating parameter of the interaction. We have shown that depending on the experimental conditions (different $\mathrm{pH}$, buffer ionic strength, membrane phase state and elastic properties) different responses of the bilayer to the PAH-coated AuNPs can be observed: they ranged from no change to the bilayer to complete disruption and removal of the 
bilayer from the surface. Furthermore, we have shown that AuNPs induce structural changes of vesicles depending on $\mathrm{pH}$ conditions: budding, tube formation, or disruption of vesicles.

In the presented work we have attempted to improve our understanding of the structure of charged two-component lipid membranes and to give insights into the mechanisms behind colloidmembrane interactions. 


\section{APPENDIX A}

\section{Fitting parameters}

TABLE 1. Fit results for various lipid mixtures in fluid phase at $23.8{ }^{\circ} \mathrm{C}$.

\begin{tabular}{|c|c|c|c|c|}
\hline Fit parameter & $\begin{array}{c}\text { DOTAP/DOPC } \\
(1: 9)\end{array}$ & $\begin{array}{c}\text { DOPC/DOPS } \\
(4: 1)\end{array}$ & DOPC & $\begin{array}{c}\text { DOTAP/DOPC }^{(1)} \\
(1: 9)\end{array}$ \\
\hline f1 & -0.92 & -0.93 & -0.70 & -0.93 \\
\hline f2 & -0.70 & -0.71 & -0.67 & -0.70 \\
\hline f3 & 0.36 & 0.40 & 0.33 & 0.36 \\
\hline f4 & -0.15 & -0.12 & -0.20 & -0.16 \\
\hline f5 & -0.28 & -0.28 & -0.36 & -0.25 \\
\hline f6 & 0.08 & 0.1 & 0.1 & 0.10 \\
\hline $\mathrm{f7}$ & -0.1 & -0.09 & -0.06 & -0.1 \\
\hline f8 & 0.05 & 0.04 & 0.02 & 0.05 \\
\hline f9 & 0.05 & 0.1 & 0.11 & 0.09 \\
\hline $\mathrm{d} 0$ & $23.32 \pm 0.12$ & $23.56 \pm 0.10$ & $23.05 \pm 0.18$ & $23.98 \pm 0.63$ \\
\hline $\mathrm{qc}$ & $0.0245 \pm 0.0003$ & $0.0242 \pm 0.0006$ & $0.0238 \pm 0.0001$ & $0.0217 \pm 0.0001$ \\
\hline$\Delta_{\max }$ & $0.16 \pm 0.01$ & $0.18 \pm 0.02$ & $0.20 \pm 0.06$ & $0.18 \pm 0.04$ \\
\hline$\sigma$ & $2.04 \pm 0.08$ & $2.05 \pm 0.18$ & $1.40 \pm 0.66$ & $2.04 \pm 0.47$ \\
\hline
\end{tabular}

Fit results for various lipid mixtures at $23.8^{\circ} \mathrm{C}$ (also see Fig 5.3.1). $\left({ }^{1}\right.$ ) are the values for D8 Advance reflectometer measurements.

TABLE 2. Fit results for various lipid mixtures in gel phase at $23.8^{\circ} \mathrm{C}$.

\begin{tabular}{cccc}
\hline Fit parameter & $\begin{array}{c}\text { DPPC/DPPS } \\
(4: 1)\end{array}$ & $\begin{array}{c}\text { DPPC/DPPS } \\
(1: 1)\end{array}$ & $\begin{array}{c}\text { DPPC/DPPS } \\
(1)\end{array}$ \\
\hline f1 & -0.75 & -0.75 & -0.7 \\
f2 & -0.36 & -0.19 & -0.18 \\
f3 & 0.28 & 0.11 & 0.14 \\
f4 & -0.4 & -0.2 & -0.08 \\
f5 & -0.07 & 0.01 & 0.06 \\
f6 & -0.1 & -0.05 & -0.04 \\
f7 & 0.02 & 0.03 & 0.02 \\
f8 & 0.01 & -0.05 & -0.04 \\
f9 & -0.05 & 0.05 & 0.04 \\
d0 & $28.26 \pm 0.34$ & $29.8 \pm 0.06$ & $28.74 \pm 0.40$ \\
qc & $0.0233 \pm 0.0007$ & $0.0237 \pm 0.0001$ & $0.0216 \pm 0.0004$ \\
$\Delta_{\max }$ & $0.27 \pm 0.02$ & $0.38 \pm 0.01$ & $0.35 \pm 0.04$ \\
$\sigma$ & $2.46 \pm 0.10$ & $2.09 \pm 0.14$ & $5.42 \pm 0.28$ \\
\hline
\end{tabular}

Fit results for various lipid mixtures at $23.8^{\circ} \mathrm{C}$ (also see Fig 5.3.1). $\left({ }^{1}\right)$ are the values for $\mathrm{D} 8$ Advance reflectometer measurements. 
A. FITTING PARAMETERS 


\section{APPENDIX B}

\section{Igor Pro procedures}

\#pragma rtGlobals=1 // Use modern global access method.

Macro data()

Rename wave0,hgl;

Rename wave 1, cyb0;

Rename wave2,filter:

Rename wave 3, mon3;

Make/N=98/D wave4, wave5, wave6, wave7

AppendToTable wave4, wave5, wave6, wave7

Rename wave4,qz;

qz $:=4 * \mathrm{pi} / 0.922 *(\sin (\mathrm{pi} * \mathrm{hgl} /(180)))$

Rename wave5, DetError;

DetError : $=\operatorname{sqrt}($ cyb0)

Rename wave6,DetFilter;

DetFilter $:=c y b 0 /\left(0.43612^{\sim}\right.$ filter $)$

Rename wave7,DetFilterError;

DetFilterError=DetError/(0.43612^filter)

Display DetFilter vs qz

ErrorBars DetFilter Y, wave=(DetFilterError,DetFilterError)

ModifyGraph $\log ($ left $)=1$

Label left "R"; DelayUpdate

Label bottom "Qz $[\mathrm{A} \backslash \backslash \mathrm{S}-1 \backslash \backslash \mathrm{M}]$ "

ModifyGraph mode=3, marker $=8, \operatorname{msize}=2, r g b=(0,0,0)$

ModifyGraph nticks (bottom) $=8$

END

\#pragma rtGlobals $=1$

// Use modern global access method.

macro offset()

Make/N=98/D wave20, wave21 
AppendToTable wave20, wave21

Rename wave20, Fit0ffset

Rename wave21, OffsetError

Make/N=26/D wave22, wave23

AppendToTable wave22, wave23

CurveFit poly 3 , wave1/X=wave0 / $\mathrm{D}=$ wave22 $/ \mathrm{R}=$ wave23

fitoffset $=W_{\text {_coef }}[0]+W_{-} \operatorname{coef}[1] * h g l+W_{\text {_coef }}[2] *$ hgl 2

OffsetError=sqrt (fitoffset)

\#0ffsetError=sqrt(W_sigma[0]^2 + (hgl*W_sigma[1]) 2

$+($ hgl^2*W_sigma $[2]) \sim 2)$

AppendToGraph Fit0ffset vs qz

ErrorBars Fit0ffset $Y$, wave=(0ffsetError, OffsetError)

Display wave22 vs wave0

ModifyGraph $\log (\operatorname{left})=1$

AppendToGraph wave 1 vs wave 0

END

\#pragma rtGlobals=1 // Use modern global access method.

macro illum()

Make/N=98/D wave8, wave9, wave10, wave11, wave12

AppendToTable wave8, wave9, wave10, wave11, wave12

Rename wave8, IllCorr

illCorr := DetFilter*0.115/(10*sin $(\mathrm{pi} * \mathrm{hgl} / 180)) *(\mathrm{hgl}[\mathrm{x}]<=180 * \operatorname{asin}(0.115 / 10) / \mathrm{pi})$

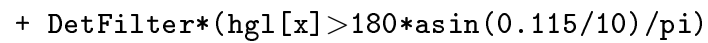

Rename wave9, Det0ffset

DetOffset $=$ IllCorr -Fit0ffset

Rename wave10, Det0ffsetError

Det0ffsetError=sqrt (DetFilterError^2 + OffsetError^2)

Display Det0ffset vs qz

ErrorBars Det0ffset Y, wave=(Det0ffsetError, Det0ffsetError)

ModifyGraph $\log ($ lef $t)=1$

Label left "R";DelayUpdate

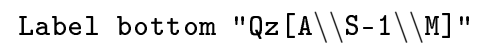

ModifyGraph mode $=3$, marker $=8, \mathrm{msize}=2, \mathrm{rgb}=(0,0,0)$ 
ModifyGraph nticks (bottom) $=8$

Rename wave11, NormIntensity

NormIntensity=Det0ffset/4.27e9

Rename wave12, NormIntensityError

NormIntensityError=Det0ff setError/4.27e9

Display NormIntensity vs qz

ErrorBars NormIntensity $\mathrm{Y}$, wave=(NormIntensityError, NormIntensityError)

ModifyGraph $\log ($ left $)=1$

Label left "R";DelayUpdate

Label bottom "Qz $[\mathrm{A} \backslash \backslash \mathrm{S}-1 \backslash \backslash \mathrm{M}]$ "

ModifyGraph mode $=3$, marker $=8, \mathrm{msize}=2, \mathrm{rgb}=(0,0,0)$

ModifyGraph nticks (bottom) $=8$

Save/J/M=" $\backslash r \backslash n$ " $/ \mathrm{P}=$ IgorPro $q z$, NormIntensity, NormIntensityError as "DPPCDPPS8020_norm.dat"

END 



\section{APPENDIX C}

\section{Program listing}

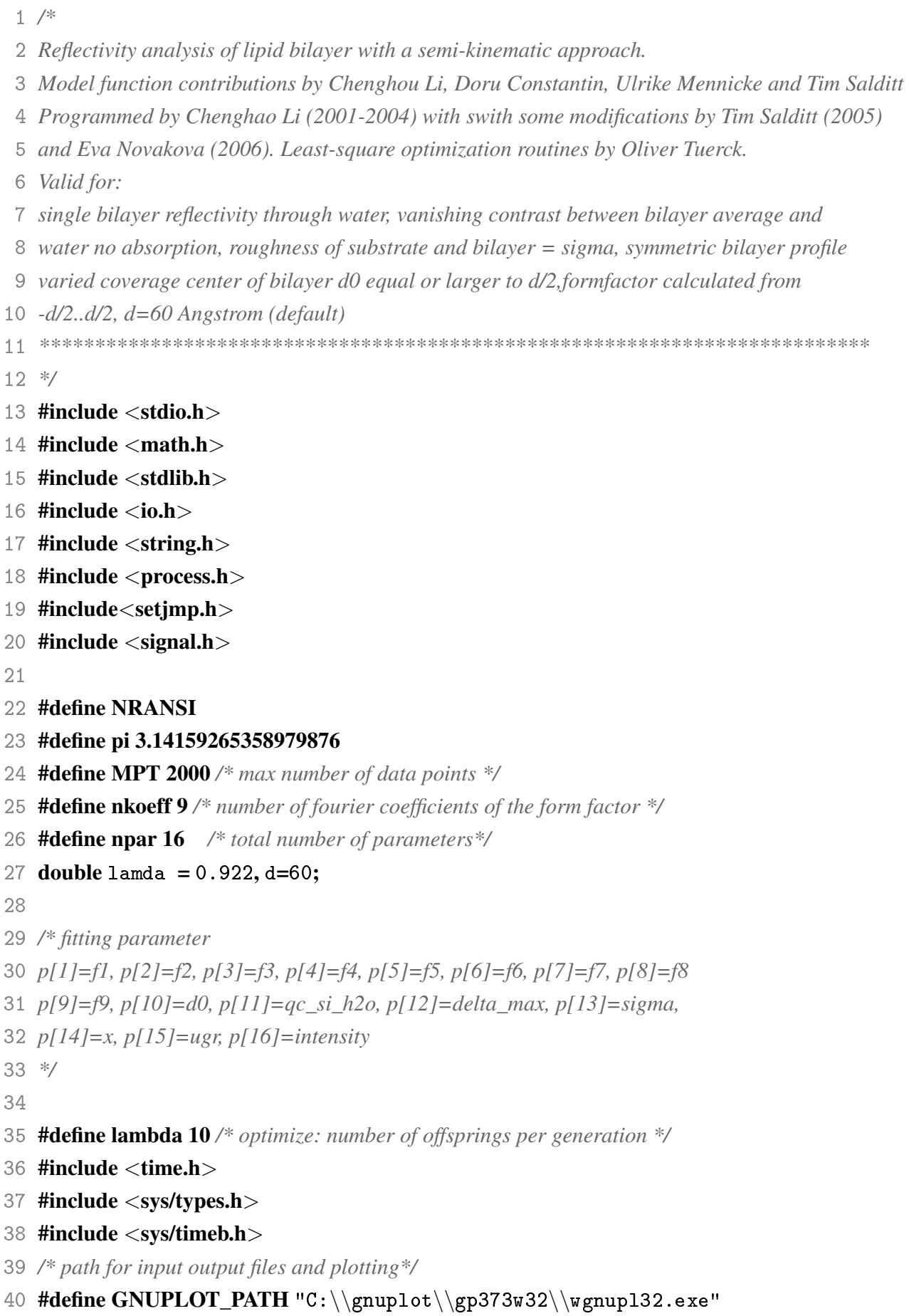




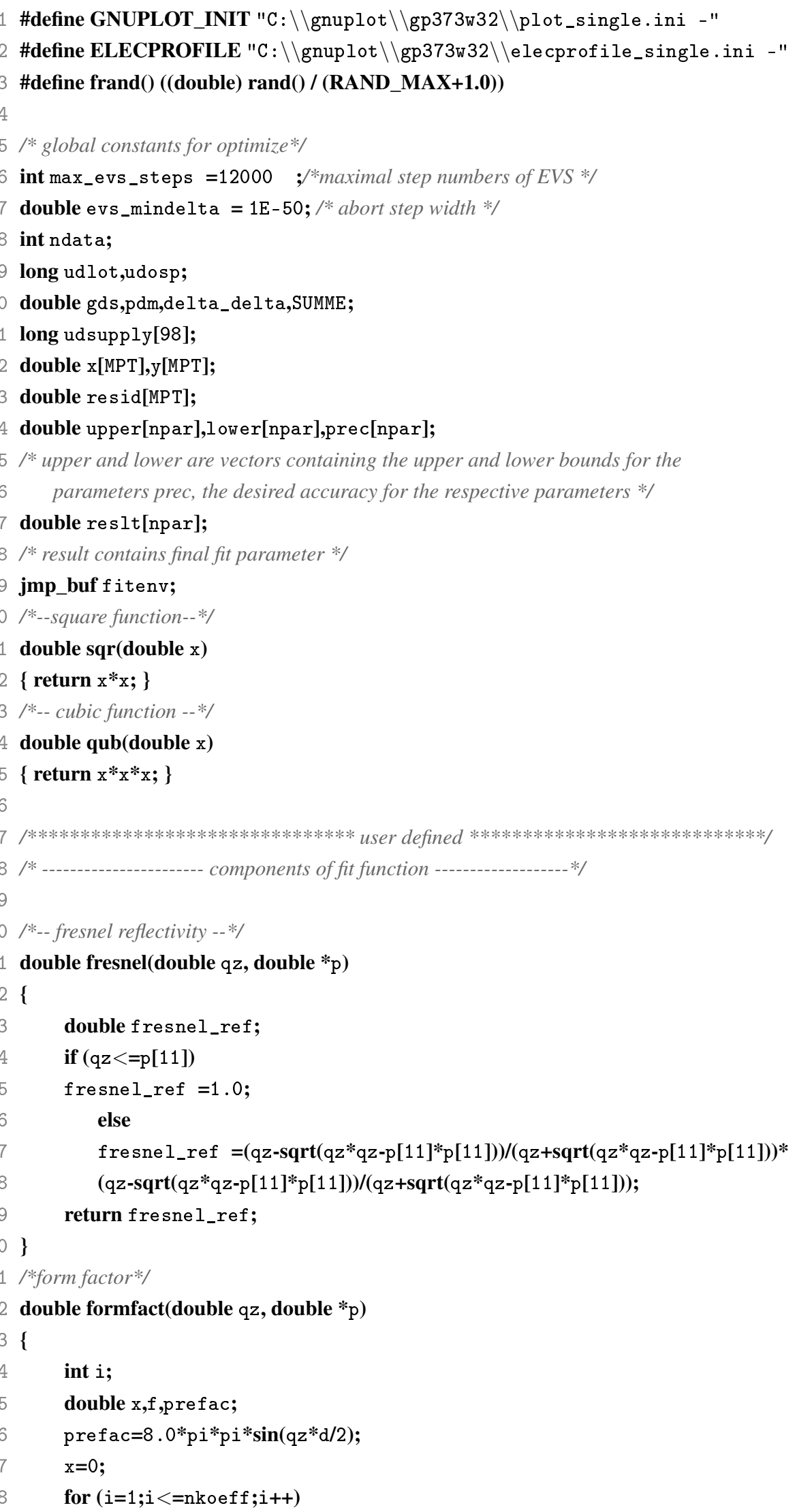




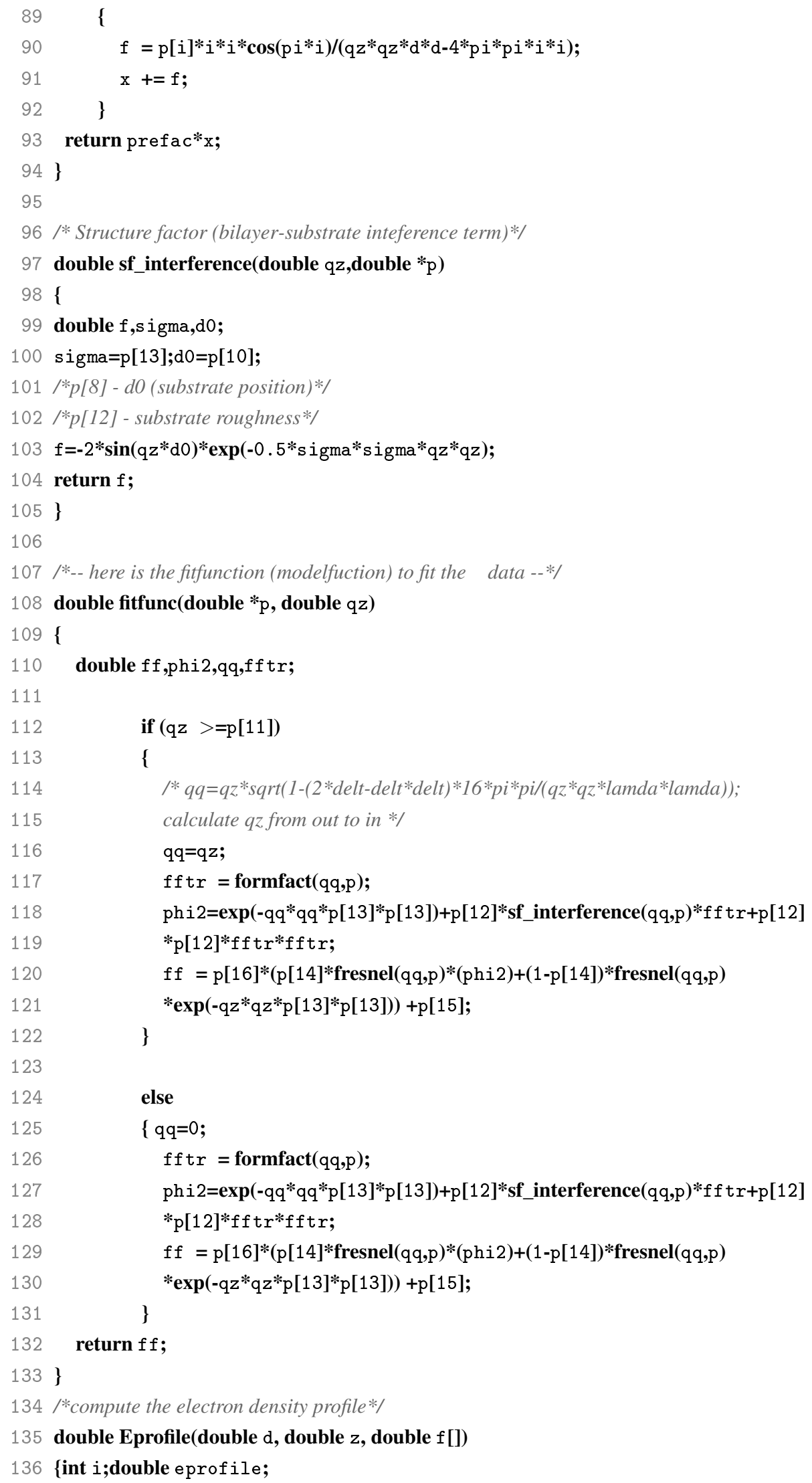




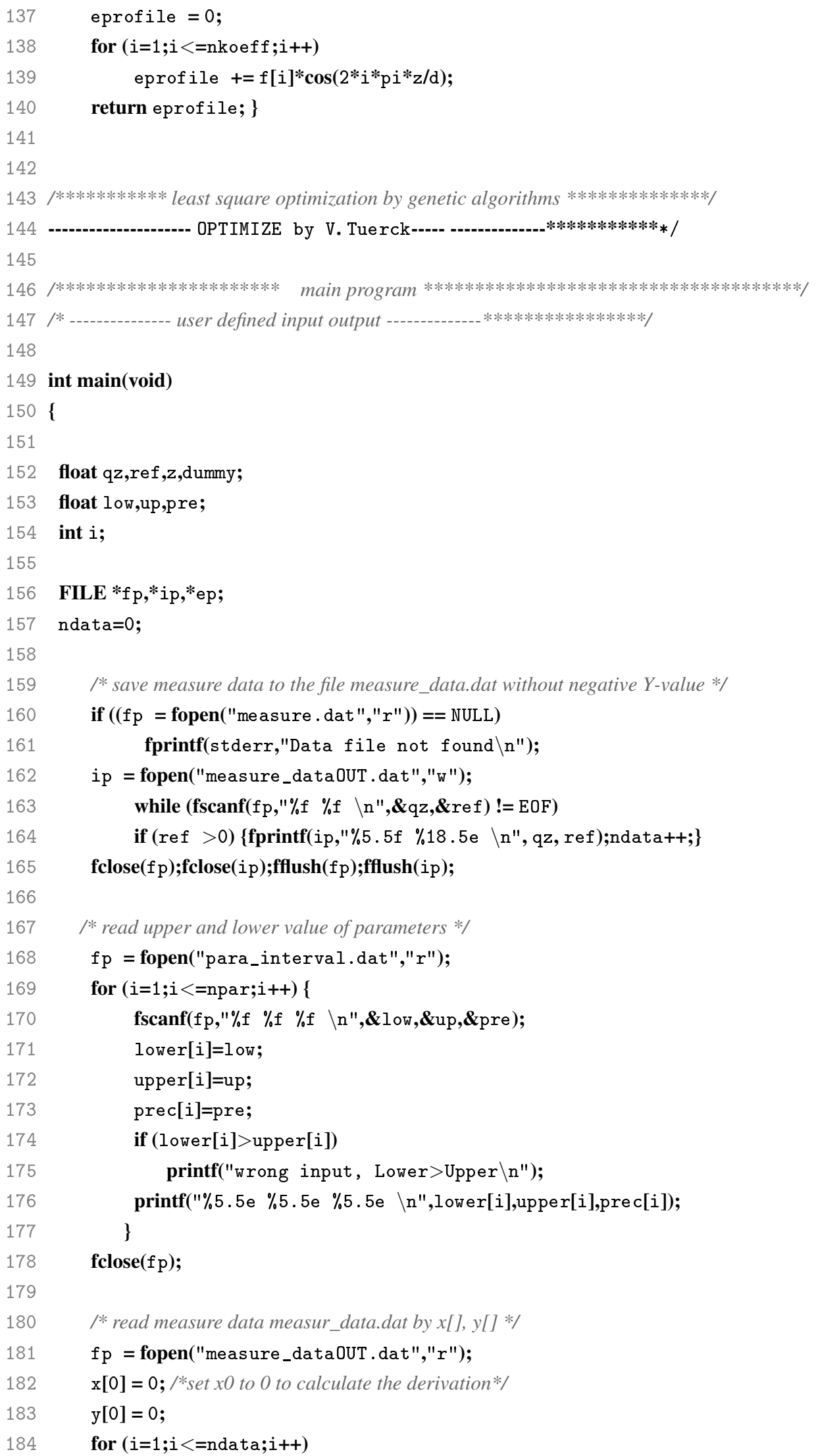




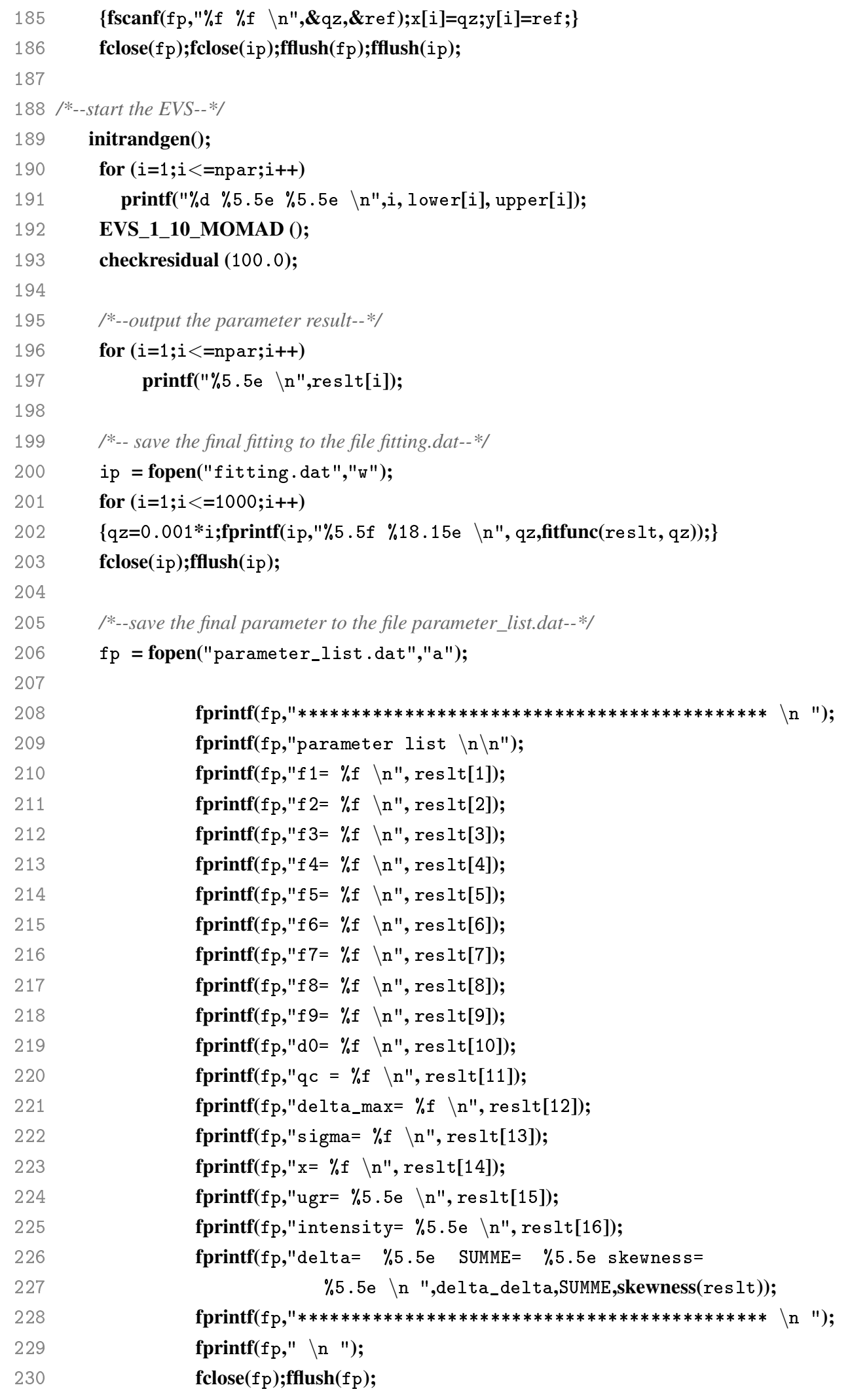


$233 /$ save the e-profile to the file eprofile.dat*/

234 ep = fopen("eprofile.dat","w");

235 for $(i=1 ; i<=1500 ; i++)$

$236\left\{z=-d+0.1 *_{i} ;\right.$ fprintf(ep," $\% 5.5 f \% 18.15 e \backslash n ", z$,

237 reslt $[12] * 0.364 * \operatorname{Eprofile(d,z,reslt)+0.334);\} }$

238 fclose(ep);fflush(ep);

239

240

241

242

\#undef NRANSI

$243\}$

return 0 ; 


\section{List of abbreviations}

$\mathrm{T}_{m}$ - phase transition temperature

$L_{c}$ - ordered sub-gel phase

$L_{\beta}$ - phase gel phase

$P_{\beta^{\prime}}$ - phase ripple phase

$L_{\alpha}$ - phase liquid-crystalline phase

PC - phosphatidylcholine

SM - sphingomyelin

PE - phosphatidylethanolamine

PS - phosphatidylserine

PA - phosphatidic acid

PG - phosphatidylglycerol

PI - phosphatidylinositol

SLB - supported lipid bilayer

DPPS - 1,2-Dipalmitoyl-sn-Glycero-3-Phosphatidylserine

DOTAP - 1,2-Dioleoyl-3-Trimethylammonium-Propane

DOPC - 1,2-Dioleoyl-sn-Glycero-3-Phosphatidylcholine

DOPS - 1,2-Dioleoyl-sn-Glycero-3-Phosphatidylserine

DPPC - 1,2-Dipalmitoyl-sn-Glycero-3-Phosphatidylcholine

DMPC - 1,2-Dimyristoyl-3-Phosphatidylcholin

Texas red-DHPE - N-(Texas Red sulfonyl)-1,2-dihexadecanoyl-snglycero-3-phosphoethanolamine, triethylammonium salt

AFM - Atomic force microscopy

PSS - poly(sodium-4-styrene sulfonate)

PAH - poly(allylamine) hydrochloride

FT-IR - Fourier transform infrared

GUV - giant unilamellar vesicle

DLVO - Derjaguin-Landau-Verwey-Overbeek theory

FWHM - full width at half maximum

NEXAFS - near-edge X-ray absorption fine structure

XAS - X-ray absorption spectroscopy

OD - optical density

MO - molecular orbital theory

XANES - X-ray absorption near-edge structure

OSA - order sorting aperture 
STXM - scanning transmission X-ray microscope

FZP - Fresnel zone plate

PS - polystyrene

AuNP - gold nanoparticle 


\section{Bibliography}

[1] Genis, R. B., Biomembranes - molecular structure and function, Springer - Verlag, Heidelberg, 1989.

[2] Fielding, C. J., editor, Lipid Rafts and Caveolae: From Membrane Biophysics to Cell, Wiley-VCH, 2007.

[3] Lucke, M., Membrane Structural Biology: With Biochemical and Biophysical Foundations, Cambridge University Press, 2008.

[4] Cevc, G., Strohmaier, L., Berkholz, J., and Blume, G., Molecular mechanism of protein interactions with the lipid bilayer membrane, Stud. Biophys. 138 (1990) 57.

[5] Heimburg, T. and Marsh, D., Protein surface-distribution and protein-protein interactions in the binding of peripherial proteins to charged lipid membranes, Biophys. J. 68 (1995) 5436.

[6] von Heijne, G., Membrane protein assembly: the rules of the game, Bioessays 17 (1995) 25.

[7] Ghosh, A., Rukmini, R., and Chattopadhyay, A., Modulation of tryptophan environment in membrane-bound melittin by negatively charged phospholipids: implications in membrane organization and function, Biochemistry 36 (1997) 14291.

[8] Bernardes, C., Valdeira, M., and Ramalho-Santos, J., Fusion activity of African swine fever virus towards target membranes: lipid dependence and effect of dehydrating agents, Biochem. Mol. Biol. Inter. 37 (1995) 481.

[9] Tuma, P., Stachniak, M., and Collins, C., Activation of dynamin GTPase by acidic phospholipids and endogenous rat brain vesicles, J. Biol. Chem. 268 (1993) 17240.

[10] Barylko, B. et al., Synergistic activation of dynamin GTPase by Grb2 and phosphoinositides, J. Biol. Chem. 273 (1998) 3791.

[11] Green, W. and Andersen, O., Surface charges and ion channel function, Annu. Rev. Physiol. 53 (1991) 341.

[12] Van der Goot, F., Didat, N., Pattus, F., Dowhan, W., and Letellier, L., Role of acidic lipids in the translocation and channel activity of colicin-A and colicin-N in Escherichia coli cells, Eur. J. Biochem. 213 (1993) 217.

[13] Tamm, L. K. and McConnell, H. M., Supported phospholipid bilayers., Biophys. J. 47 (1985) 105.

[14] Dimitrov, D. S., Interactions of antibody-conjugated nanoparticles with biological surfaces, Colloids and Surfaces A: Physicochem. Eng. Aspects 282283 (2006) 8.

[15] Ramos, L., Lubensky, T. C., Dan, N., Nelson, P., and Weitz, D. A., Surfactant-Mediated Two-Dimensional Crystallization of Colloidal Crystals, Science 286 (1999) 2325.

[16] Hong, S. et al., Physical interactions of nanoparticles with biological membranes: The observation of nanoscale hole formation, Journal of Chemical Health and Safety 13 (2006) 16.

[17] May, S., Stability of macroion-decorated lipid membranes, J. Phys.: Condens. Matter 17 (2005) R833.

[18] Stöhr, J., NEXAFS Spectroscopy, Springer-Verlag, Berlin, 1992.

[19] Singer, S. and Nicholson, G., Fluid mosaic model of structure of cell-membrane, Science 175 (1972) 720.

[20] Cevc, G., editor, Phospholipids Handbook, Marcel Dekker, INC., New York, 2 edition, 1993.

[21] Sackmann, E., Biological Membranes Architecture and Function in Handbook of Biological Physics, Volume 1, Elsevier Science B.V., 1995.

[22] Axelrod, D. and Wang, M. D., Reduction-of-dimensionality kinetics at reaction-limited cell surface receptors, Biophys. J. 66 (1994) 588.

[23] Langner, M. and Kubica, K., The electrostatics of lipid surfaces, Chemistry and Physics of Lipids 101(1) (1999) 3.

[24] Koynova, R. and Caffrey, M., Phases and phase transitions of the phosphatidylcholines, Biochim. Biophys. Acta 1376 (1998) 91. 
[25] Sackmann, E., Supported Membranes: Scientific and Practical Applications, Science 271 (1996) 43.

[26] Rädler, J. O., Koltover, I., Salditt, T., and Safinya, C. R., Structure of DNA-cationic liposome complexes: DNA intercalation in multilamellar membranes in distinct interhelical packing regimes, Science 275 (1997) 810.

[27] Salditt, T., Koltover, I., Rädler, J. O., and Safinya, C. R., Two-dimensional smectic ordering of linear DNA chains in self-assembled DNA-cationic liposome mixtures, Physical Review Letters 79 (1997) 2582.

[28] McIntosh, T. J. and Simon, S. A., Contributions of hydration and steric (entropic) pressures to the interactions between phosphatidylcholine bilayers: Experiments with the subgel phase, Biochemistry 32(32) (1993) 8374.

[29] Lopez Cascales, J., Berendsen, H., and de la Torre, G., Molecular dynamics simulation of water between two charged layers of dipalmitoylphosphatidylserine, J. Phys. Chem. 100 (1996) 8621.

[30] Marrink, S. and Berendsen, H., Simulation of water transport through a lipid membrane, J. Phys. Chem. 98 (1994) 4155.

[31] Bennett, M., Gibson, D., Schwartz, S., and Tait, J., Binding and phagocytosis of apoptotic vascular smooth muscle cells is mediated in part by exposure of phosphatidylserine, Circ. Res. 77 (1995) 1136.

[32] Gouy, G., Sur la constitution de la charge electrique a la surface d'un electrolyte, Ann. Phys. 4 (1910) 457.

[33] Chapman, D., A contribution to the theory of electrocapillarity, Philos. Mag. (1913) 475.

[34] Debye, P. and Hückel, E., Zur Theorie der Elektrolyte. I. Gefrierpunktserniedrigung und verwandte Erscheinungen, Phyzik Z. 24 (1923) 185.

[35] Andelman, D., Electrostatic Properties of Membranes: The Poisson-Boltzmann Theory in Handbook of Biological Physics Volume 1, Elsevier Science B.V., 1995.

[36] Winterhalter, M. and Helfrich, W., Bending elasticity of electrical charged bilayers: coupled monolayers, neutral surfaces, and balancing stresses, J. Phys. Chem. 96 (1992) 327.

[37] Kékicheff, P., Marcelja, S., Senden, T. J., and Shubin, V. E., Charge reversal seen in electrical double layer interaction of surfaces immersed in 2:1 calcium electrolyte, J. Chem. Phys. 99 (1993) 6098.

[38] Israelashvili, J., Intermolecular and Surface Forces, Academic Press, San Diego, CA, 1985.

[39] Helfrich, W., Elastic Properties of Lipid Bilayers-Theory and Possible Experiments, Z. Naturforsch. C 28 (1973) 693.

[40] Tien, H. and Ottova-Leitmannova, A., editors, Planar Lipid Bilayers (BLM's) and Their Applications (Membrane Science and Technology), Elsevier Science, 2003.

[41] Watts, T. H., Brian, A. A., Kappler, J. W., Marrack, P., and McConnell, H. M., Antigen Presentation by Supported Planar Membranes Containing Affinity-Purified I-Ad, Proceedings of the National Academy of Sciences 81 (1984) 7564.

[42] Kalb, E., Frey, S., and Tamm, L., Formation of supported planar bilayers by fusion of vesicles to supported phospholipid monolayers, Biochim. Biophys. Acta. 1103 (1992) 307.

[43] Seifert, U., Configurations of fluid membranes and vesicles, Adv. Phys. 46 (1997) 13.

[44] Lipowsky, R. and Seifert, U., Adhesion of Vesicles and Membranes, Mol. Cryst. Liq. Cryst. 202 (1991) 17.

[45] Reviakine, I. and Brisson, A., Formation of Supported Phospholipid Bilayers from Unilamellar Vesicles Investigated by Atomic Force Microscopy, Langmuir 16 (2000) 1806.

[46] Johnson, J. M., Ha, T., Chu, S., and Boxer, S. G., Early Steps of Supported Bilayer Formation Probed by Single Vesicle Fluorescence Assays, Biophys. J. 83 (2002) 3371.

[47] Richter, R. and Brisson, A., Characterization of Lipid Bilayers and Protein Assemblies Supported on Rough Surfaces by Atomic Force Microscopy, Langmuir 19 (2003) 1632.

[48] Keller, C. A., Glasmästar, K., Zhdanov, V. P., and Kasemo, B., Formation of Supported Membranes from Vesicles, Phys. Rev. Lett. 84 (2000) 5443.

[49] Richter, R., Mukhopadhyay, A., and Brisson, A., Pathways of Lipid Vesicle Deposition on Solid Surfaces: A Combined QCM-D and AFM Study, Biophys. J. 85 (2003) 3035.

[50] Richter, R., Berat, R., and Brisson, A., Formation of Solid-Supported Lipid Bilayers: An Integrated View, Langmuir 22 (2006) 3497. 
[51] Rossetti, F., Bally, M., Michel, R., Textor, M., and Reviakine, I., Interactions between Titanium Dioxide and Phosphatidyl Serine-Containing Liposomes: Formation and Patterning of Supported Phospholipid Bilayers on the Surface of a Medically Relevant Material, Langmuir 21 (2005) 6443.

[52] Castellana, E. T. and Cremer, P. S., Solid supported lipid bilayers: From biophysical studies to sensor design, Surface Science Reports 61(10) (2006) 429.

[53] Groves, J., Ulman, N., Cremer, P., and Boxer, S., Substrate-Membrane Interactions: Mechanisms for Imposing Patterns on a Fluid Bilayer Membrane, Langmuir 14 (1998) 3347.

[54] Rädler, J., Strey, H., and Sackmann, E., Phenomenology and Kinetics of Lipid Bilayer Spreading on Hydrophilic Surfaces, Langmuir 11(11) (1995) 4539.

[55] Cremer, P. and Boxer, S., Formation and Spreading of Lipid Bilayers on Planar Glass Supports, Journal of Physical Chemistry B 103 (1999) 2554.

[56] Garcia-Manyes, S., Oncins, G., and Sanz, F., Pages Effect of $p H$ and ionic strength on phospholipid nanomechanics and on deposition process onto hydrophilic surfaces measured by AFM, Electrochimica Acta 51(24) (2006) 5029.

[57] Ong, S., Zhao, X., and Eisenthal, K. B., Polarization of water molecules at a charged interface: second harmonic studies of the silica/water interface, Chemical Physics Letters 191(3-4) (1992) 327.

[58] Reviakine, I., Simon, A., and Brisson, A., Effect of Ca2+ on the Morphology of Mixed DPPC-DOPS Supported Phospholipid Bilayers, Langmuir 16 (2000) 1473.

[59] Reich, C., Structure, Fluidity and Phase Behavior of Supported Lipid Membranes: An Investigation by X-ray Reflectivity and Fluorescence Microscopy, $\mathrm{PhD}$ thesis, Ludwig-Maximilians-Universitaet Muenchen, 2007.

[60] Nováková, E., Giewekemeyer, K., and Salditt, T., Structure of two-component lipid membranes on solid support: An x-ray reflectivity study, Physical Review E (Statistical, Nonlinear, and Soft Matter Physics) 74 (2006) 051911.

[61] Miller, C. E., Majewski, J., Gog, T., and Kuhl, T. L., Characterization of Biological Thin Films at the SolidLiquid Interface by X-Ray Reflectivity, Physical Review Letters 94 (2005) 238104.

[62] Rossetti, F., Textor, M., and Reviakine, I., Asymmetric Distribution of Phosphatidyl Serine in Supported Phospholipid Bilayers on Titanium Dioxide, Langmuir 22 (2006) 3467.

[63] Tanaka, M. and Sackmann, E., Polymer-supported membranes as models of the cell surface, Nature 437 (2005) 656.

[64] Reich, C., Neff, P. A., Bausch, A. R., Rädler, J. O., and Nickel, B., Supported Membranes on Polyelectrolyte Layers Studied by X-ray Reflectometry, physica status solidi (a) 203 (2006) 3463.

[65] Hermanson, G. T., Bioconjugate Techniques, Academic press, INC, 1996.

[66] Derjaguin, B. V. and Landau, L. D., Acta Phys. Chim. USSR 14 (1941) 633.

[67] Verwey, E. J. W. and Overbeek, J. T. G., Theory of the Stability of Lyophobic Colloids, Elsevier, 1948.

[68] Schiller, P., Wahab, M., Bagusat, F., Reimer, U., and Mögel, H. J., Adsorption of colloidal particles on mixed membranes in Progr. Colloid Polym. Sci., volume 128, 2004.

[69] Deserno, M. and Bickel, T., Wrapping of a spherical colloid by a fluid membrane, Europhys. Lett. 62 (2003) 767.

[70] Koltover, I., Rädler, J. O., and Safinya, C. R., Membrane Mediated Attraction and Ordered Aggregation of Colloidal Particles Bound to Giant Phospholipid Vesicles, Phys. Rev. Lett. 82 (1999) 1991.

[71] Noguchi, H. and Takasu, M., Adhesion of Nanoparticles to Vesicles: A Brownian Dynamics Simulation, Biophys. J. 83 (2002) 299.

[72] Goodman, C. M., McCusker, C. D., Yilmaz, T., and Rotello, V. M., Toxicity of gold nanoparticles functionalized with cationic and anionic side chains, Bioconjug Chem. 15 (2004) 897.

[73] Silvius, J. R., Thermotropic Phase Transitions of Pure Lipids in Model Membranes and Their Modifications by Membrane ProteinsLipid-Protein Interactions, John Wiley \& Sons, Inc.New York, 1982.

[74] Käsbauer, M., Junglas, M., and Bayerl, T. M., Effect of Cationic Lipids in the Formation of Asymmetries in Supported Bilayers, Biophys J 76 (1999) 2600. 
[75] Schmidt, H. O., Characterization of silicon surface preparation processes for advanced gate dielectricsced gate dielectrics, IBM Journal of Research and Development 43 (2000) 351.

[76] Xie, A. F., Yamada, R., Gewirth, A. A., and Granick, S., Materials Science of the Gel to Fluid Phase Transition in a Supported Phospholipid Bilayer, Phys. Rev. Lett. 89 (2002) 246103.

[77] Charrier, A. and Thibaudau, F., Main Phase Transitions in Supported Lipid Single-Bilayer, Biophys. J. 89 (2005) 1094.

[78] Leckband, D. E., Helm, C. A., and Israelachvili, J., Role of Calcium in the Adhesion and Fusion of Bilayers, Biochemistry 32 (1993) 127.

[79] Jass, J., Tjärnhage, T., and Puu, G., From Liposomes to Supported, Planar Bilayer Structures on Hydrophilic and Hydrophobic Surfaces: An Atomic Force Microscopy Study, Biophys J. 79 (2000) 3153.

[80] Angelova, M. I., Liposome electroformation. In Giant Vesicles, Number 27-36, John Wiley and Sons, Chichester, UK., 2000.

[81] Als-Nielsen, J. and McMorrow, D., Elements of Modern X-Ray Physics, 2001.

[82] Born, M. and Wolf, E., Principles of Optics, Pergamon Press, Bath, 1964.

[83] Attwood, D., Soft X-Rays and Extreme Ultraviolet Radiation: Principles and Applications, Cambridge University Press, 1999.

[84] Henke, B., Gullikson, E., and Davis, J., X-ray interactions: photoabsorption, scattering, transmission, and reflection at E=50-30000 eV, Z=1-92, Atomic Data and Nuclear Data Tables 54 (2) (1993) 181.

[85] Kirz, J., Jacobsen, C., and Howells, M., X-ray microscopy and its biological applications, Quarterly Reviews of Biophysics 28(1) (1995) 33.

[86] Reich, C., Hochrein, M. B., Krause, B., and Nickel, B., A microfluidic setup for studies of solid-liquid interfaces using $x$-ray reflectivity and fluorescence microscopy, Review of Scientific Instruments 76 (2005) 095103.

[87] Sackmann, E., Supported Membranes: Scientific and Practical Applications, Science 271 (5245) (1996) 43.

[88] Maier, B. and Rädler, J. O., Conformation and Self-Diffusion of Single DNA Molecules Confined to Two Dimensions, Phys. Rev. Lett. 82 (1999) 1911.

[89] Salditt, T., Li, C., Spaar, A., and Mennicke, U., X-ray reflectivity of solid-supported, multilamellar membranes, The European Physical Journal E - Soft Matter 7(2) (2002) 105.

[90] Tolan, M., X-ray Scattering from Soft-Matter Thin Films, Springer Tracts in Modern Physics Vol. 148, Springer, Berlin, 1999.

[91] Kiessig, H., Interferenz von Röntgenstrahlen an dünnen Schichten, Ann. Phys. 10 (1931) 769.

[92] Michaelsen, C. et al., Improved graded multilayer mirrors for XRD applications, Adv. X-ray Anal. 42 (2000) 308.

[93] Brown, G. S. and Mocton, D. E., editors, Handbook on synchrotron radiation, Volume 3, North-Holland, 1991.

[94] Lequien, S., Goirand, L., and Lesimple, F., Design of the anomalous scattering beamline at the European Synchrotron Radiation Facility, Review of Scientific Instruments-5. International Conference on Synchrotron Radiation Instrumentation - New York, USA 66 (1995) 1725.

[95] Cabailh, G. et al., Synchrotron radiation studies of the growth and beam damage of tin-phthalocyanine on GaAs $(0$ 0 1)-1 x 6 substrates, Applied Surface Science 234(1-4) (2004) 144.

[96] Sivia, D. S., Data Analysis: A Bayesian Tutorial (Oxford Science Publications), Oxford University Press, 1996.

[97] Koenig, B. et al., Neutron Reflectivity and Atomic Force Microscopy Studies of a Lipid Bilayer in Water Adsorbed to the Surface of a Silicon Single Crystal, Langmuir 12 (1996) 1343.

[98] Pabst, G., Rappolt, M., Amenitsch, H., and Laggner, P., Structural information from multilamellar liposomes at full hydration: Full q-range fitting with high quality x-ray data, Phys. Rev. E 62 (2000) 4000.

[99] Wiener, M. C., Suter, R. M., and Nagle, J. F., Structure of the fully hydrated gel phase of dipalmitoylphosphatidylcholine., Biophys. J. 55 (1989) 315.

[100] Liu, Y. and Nagle, J. F., Diffuse scattering provides material parameters and electron density profiles of biomembranes, Phys. Rev. E 69 (2004) 040901. 
[101] Leonenko, Z. V., Finot, E., Ma, H., Dahms, T. E. S., and Cramb, D. T., Investigation of Temperature-Induced Phase Transitions in DOPC and DPPC Phospholipid Bilayers Using Temperature-Controlled Scanning Force Microscopy, Biophys. J. 86 (2004) 3783.

[102] Hochrein, M., Reich, C., Krause, B., Rädler, J., and Nickel, B., Structure and Mobility of Lipid Membranes on a Thermoplastic Substrate, Langmuir 22 (2006) 538.

[103] Petrache, H. I. et al., Structure and Fluctuations of Charged Phosphatidylserine Bilayers in the Absence of Salt, Biophys. J. 86 (2004) 1574.

[104] Yang, L. et al., Self-assembled virus-membrane complexes, Nature Materials 3 (2004) 615 .

[105] Zuidam, N. J. and Barenholz, Y., Electrostatic parameters of cationic liposomes commonly used for gene delivery as determined by 4-heptadecyl-7-hydroxycoumarin, Biochim. Biophys. Acta 1329 (1997) 211.

[106] Ohnishi, S. and Ito, T., Calcium-induced phase separations in phosphatidylserine-phosphatidylcholine membranes, Biochemistry 13(5) (1974) 881.

[107] Jacobson, K. and Papahadjopoulos, D., Phase transitions and phase separations in phospholipid membranes induced by changes in temperature, $p H$, and concentration of bivalent cations, Biochemistry 14(1) (1975) 152.

[108] Ross, M., Steinem, C., Galla, H. J., and Janshoff, A., Visualization of Chemical and Physical Properties of Calcium-Induced Domains in DPPC/DPPS Langmuir-Blodgett Layers, Langmuir 17 (2001) 2437.

[109] Braun, C., The Reflectivity Tool. Parratt 32, HMI, Berlin, Germany (1999).

[110] Lide, D., editor, Handbook of Chemistry and Physics-Ed. vol. 74, CRC Press, London, 1993-1994.

[111] Sohling, U. and Schouten, A. J., Investigation of the Adsorption of Dioleoyl-L-r-phosphatidic Acid Mono- and Bilayers from Vesicle Solution onto Polyethylenimine-Covered Substrates, Langmuir 12 (1996) 3912.

[112] Constantin, D., Mennicke, U., Li, C., and Salditt, T., Solid supported lipid multilayers: Structure factor and fluctuations, Eur. Phys. J. E 12 (2003) 283.

[113] Salditt, T., Lipid-peptide interaction in oriented bilayers probed by interface-sensitive scattering methods, Current Opinion in Structural Biology 13(4) (2003) 467.

[114] Lyatskaya, Y., Liu, Y., Tristram-Nagle, S., Katsaras, J., and Nagle, J. F., Method for obtaining structure and interactions from oriented lipid bilayers, Phys. Rev. E 63 (2000) 011907.

[115] Fragneto, G. et al., A fluid floating bilayer, EPL (Europhysics Letters) 53 (2001) 100.

[116] Daillant, J. et al., Structure and fluctuations of a single floating lipid bilayer, Proceedings of the National Academy of Sciences 102 (2005) 11639.

[117] Hitchcock, A. P., Morin, C., Heng, Y. M., Cornelius, R. M., and Brash, J. L., Towards practical soft X-ray spectromicroscopy of biomaterials, Journal of Biomaterials Science, Polymer Edition 13 (2002) 919.

[118] Urquhart, S. and Ade, H., Trends in the Carbonyl Core (C 1S, O IS) $\rightarrow \pi^{*}(C=O)$ Transition in the Near-Edge $X$-ray Absorption Fine Structure Spectra of Organic Molecules, Journal of Physical Chemistry B 106 (2002) 8531.

[119] Mitrea, G., Thieme, J., Guttmann, P., Heim, S., and Gleber, S., X-ray spectromicroscopy with the scanning transmission X-ray microscope at BESSY II, J. Synchrotron Rad. 15 (2008).

[120] Peth, C., Barkusky, F., and Mann, K., Near-edge x-ray absorption fine structure measurements using a laboratory-scale XUV source, J. Phys. D: Appl. Phys. 41 (2008) 105202.

[121] Jackson, J. D., Classical Electrodynamics, Wiley, New York, 1962.

[122] Levine, I., Molecular Spectroscopy, MTI Press, Boston, 1986.

[123] Lee, P. A., Citrin, P. H., Eisenberger, P., and Kincaid, B. M., Extended x-ray absorption fine structure-its strengths and limitations as a structural tool, Rev. Mod. Phys. 53 (1981) 769.

[124] Hitchcock, A. P., Soft X-ray spectromicroscopy of polymers and biopolymer interfaces, J. Synchrotron Rad. 8 (2001) 66.

[125] Kirz, J., Jacobsen, C., and Howells, M., Soft X-ray microscopes and their biological applications, Q Rev Biophys. 28(1) (1995) 33.

[126] Schmahl, G. and Rudolph, D., Lichtstarke Zonenplatten als abbildende Systeme für weiche Röontgenstrahlung, Optik 29 (1969) 577. 
[127] Niemann, B., Rudolph, D., and Schmahl, G., Soft X-ray imaging zone plates with large zone numbers for microscopic and spectroscopic applications, Optics Communications 12 (1974) 160.

[128] Michette, A. G., Optical Systems for Soft X Rays, Plenum, New York, 1986.

[129] Thieme, J., Theoretical investigations of imaging properties of zone plates and zone plate systems using diffraction theory, D. Sayre, M. R. Howells, J. Kirz, and H. Rarback, editors. X-ray Microscopy II, volume 56 of Springer Series in Optical Sciences, Berlin. Springer-Verlag. (1988) 70.

[130] Wiesemann, U. et al., The New Scanning Transmission X-Ray Microscope at BESSY II, W. Meyer-Ilse, T. Warwick, D. Attwood (Hrsg.): X-ray Microscopy, Proceedings of the Sixth International Conference, Berkeley, CA 1999, AIP Conference Proceedings 507 (2000) 430.

[131] Wiesemann, U., The Scanning Transmission X-Ray Microscope at BESSY II, PhD thesis, Georg-August University Gättingen, Germany, 2003.

[132] Rehbein, S., Nanofabrication of diffractive optics for soft X-ray and atom beam focusing, J. Phys. IV France 104 (2003) 207.

[133] Strüder, L. et al., The MPI/AIT X-ray imager (MAXI)-High speed pn CCDs for X-ray detection, Nuclear Instruments and Methods A 288 (1990) 227.

[134] Niemann, B., Schneider, G., Guttmann, P., and Schmahl, G., The new Göttingen X-ray microscope with object. holder in air for wet specimens, X-ray Microscopy IV, edited by V. V. Aristov and A. I. Erko, Moscow: Bogorodskii Pechatnik (1994) p. 3035.

[135] Kranzusch, S., Peth, C., and Mann, K., Spatial characterization of extreme ultraviolet plasmas generated by laser excitation of xenon gas targets, Rev. Sci. Instr. 74 (2003) 969.

[136] Buckley, C. J., The measuring and mapping of calcium in mineralized tissues by absoption difference mapping, Rev Sci. Instrum. 66 (1994) 1318.

[137] Neuhäusler, U., Abend, S., Jacobsen, C., and Lagaly, G., Soft x-ray spectromicroscopy on solid stabilized emulsions, Colloid Polym Sci 277 (1999) 719.

[138] Jacobsen, C. et al., Diffraction-limited imaging in a scanning transmission x-ray microscope, Optics Communications 86 (1991) 351.

[139] Gleber, G., Thieme, J., Niemeyer, J., and Feser, M., Interaction of organic substances with iron studied by O1s spectroscopy -Development of an analysis program, Journal de Physique IV 104 (2003) 429.

[140] Dhez, O., H.Ade, and Urquhart, S., Calibrated NEXAFS Spectra of Some Common Polymers, Electron Spectrosc. Relat. Phenom. 128 (2003) 85.

[141] Beetz, T. and Jacobsen, C., Soft X-ray radiation-damage studies in PMMA using a cryo-STXM, J. Synchrotron Rad. 10 (2002) 280.

[142] Zhang, X., Jacobsen, C., Lindaas, S., and Williams, S., Exposure strategies for PMMA in situ x-ray absorption near edge structure spectroscopy, J. Vac. Sci. Technol. B 13 (1995) 1477.

[143] Ishii, I. and Hitchcock, A., The oscillator strengths for carbon-1s and oxygen-1s excitation of some saturated and unsaturated organic alcohols, acids and esters, J. Electr. Spectrosc. Relat. Phenom. 46 (1988) 55.

[144] Kaznacheyev, K. et al., Innershell Absorption Spectroscopy of Amino Acids, J. Phys. Chem. A 106 (2002) 3153.

[145] Ramos, L., Lubensky, T. C., Dan, N., Nelson, P., and Weitz, D. A., Surfactant-mediated two-dimensional crystallization of colloidal crystals, Science 286 (1999) 2325.

[146] Leal, C., D. S. P. N. and Topgaard, D., Probing local and translational dynamics in DNA-lipid assemblies monitored by solid-state and diffusion NMR, Biochim. Biophys. Acta. 1778 (2008) 214.

[147] Denisov, G., Wanaski, S., Luan, P., Glaser, M., and McLaughlin, S., Binding of Basic Peptides to Membranes Produces Lateral Domains Enriched in the Acidic Lipids Phosphatidylserine and Phosphatidylinositol 4,5Bisphosphate: An Electrostatic Model and Experimental Results, Biophys. J. 74 (1998) 731.

[148] Harries, D., May, S., and Ben-Shaul., A., Adsorption of charged macromolecules on mixed fluid membranes, Colloid Surface A. 208 (2002) 41.

[149] Mbamala, E. C., Ben-Shaul, A., and May, S., Domain formation induced by the adsorption of charged proteins on mixed lipid membranes, Biophys. J. 88 (2005) 1702. 
[150] Tzlil, S. and Ben-Shaul, A., Flexible charged macromolecules on mixed fluid lipid membranes: theory and Monte Carlo simulations, Biophys. J. 89 (2005) 2972.

[151] Dias, R. S. and Linse, P., Colloid Adsorption onto Responsive Membranes, Biophys. J. 94 (2008) 3760.

[152] Helm, C. A., Laxhuber, L., Lösche1, M., and Möhwald1, H., Electrostatic interactions in phospholipid membranes I: Influence of monovalent ions, Colloid and Polymer Science 264 (1986).

[153] Fleck, C. C. and Netz, R. R., Electrostatic colloid-membrane binding, Europhys. Lett. 67 (314-320) 314.

[154] Jülicher, F. and Lipowsky, R., Domain-induced budding of vesicles, Phys. Rev. Lett. 70 (1993) 2964.

[155] Gozdz, W., Deformations of Lipid Vesicles Induced by Attached Spherical Particles, Langmuir 23 (2007) 5665.

[156] Rodriguez-Fernandez, J., Pastoriza-Santos, I., Perez-Juste, J., Garcia de Abajo, F., and Liz-Marzan, L., The Effect of Silica Coating on the Optical Response of Sub-micrometer Gold Spheres, Journal of Physical Chemistry C 111 (2007) 13361.

[157] Shipway, A. N., Katz, E., and Willner, I., Nanoparticle Arrays on Surfaces for Electronic, Optical, and Sensor Applications, ChemPhysChem 1 (2000) 18.

[158] Tsui, F. C., Ojcius, D. M., and Hubbell, W. L., The intrinsic pKa values for phosphatidylserine and phosphatidylethanolamine in phosphatidylcholine host bilayers, Biophys J. 49 (1986) 459.

[159] Dluhy, R. A., Cameron, D. G., Mantsch, H., and Mendelsohn, R., Fourier transform infrared spectroscopic studies of the effect of calcium ions on phosphatidylserine, Biochemistry 22 (1983) 6318.

[160] Lewis, R. N. A. H. and McElhaney, R. N., Behavior of Lipid Bilayer Model Membranes Composed of a Homologous Series of Linear Saturated Phosphatidylserines, Biophysical Journal 79 (2000) 2043.

[161] Tamm, L. K. and Tatulian, S. A., Infrared spectroscopy of proteins and peptides in lipid bilayers, Quarterly Reviews of Biophysics 30 (1997) 365.

[162] Gomez-Fernandez, J. and Villalan, J., The use of FT-IR for quantitative studies of the apparent pKa of lipid carboxyl groups and the dehydration degree of the phosphate group of phospholipids, Chemistry and Physics of Lipids 96 (1998) 41.

[163] Grahame, D. C., The electrical double layer and the theory of electrocapillarity, Chem. Rev. 41 (1947) 441.

[164] Matinyan, N. S., Ershler, I. A., and Abidor, I. G., Proton equilibrium on the surfaces of bilayer lipid membranes, Biol. Memr. (Russian) 1 (1985) 451.

[165] Mac Donald, R. C., Simon, S. A., and Baer, E., Ionic influences of the phase transition of dipalmitoylphosphatidylserine, Biochemistry 15 (1976) 885.

[166] Moncelli, M. R., Becucci, L., and Guidelli, R., The intrinsic pKa values for phosphatidylcholine, phosphatidylethanolamine, and phosphatidylserine in monolayers deposited on mercury electrodes, Biophys. J. 66 (1994) 1969.

[167] Egorova, E. M., Dissociation constants of lipid ionizable groups I. Corrected values for two anionic lipids, Colloids and Surfaces A: Physicochemical and Engineering Aspects 131 (1998) 7.

[168] Vaz, W. L. C., Nicksch, A., and Jahnig, F., Electrostatic Interactions at Charged Lipid Membranes. Measurement of Surface pH with Fluorescent Lipoid pH Indicators, European Journal of Biochemistry 83 (1978) 299.

[169] Petrov, A., Antipov, A., and Sukhorukov, G., Base-Acid Equilibria in Polyelectrolyte Systems: From Weak Polyelectrolytes to Interpolyelectrolyte Complexes and Multilayered Polyelectrolyte Shells, Macromolecules 36 (2003) 10079.

[170] Itano, K., Choi, J., and Rubner, M. F., Mechanism of the pH-Induced Discontinuous Swelling/Deswelling Transitions of Poly(allylamine hydrochloride)-Containing Polyelectrolyte Multilayer Films, Macromolecules 38 (2005) 3450 .

[171] Baumgart, T., Hess, S. T., and Webb, W. W., Imaging coexisting fluid domains in biomembrane models coupling curvature and line tension, Nature 425 (2003) 821.

[172] Parthasarathy, R. and Groves, J. T., Curvature and spatial organization in biological membranes, Soft Matter 3 (2007) 24.

[173] Rossetti, F. F. et al., Interaction of Poly(L-Lysine)-g-Poly(Ethylene Glycol) with Supported Phospholipid Bilayers, Biophysical Journal 87 (2004) 1711. 
[174] Steinem, C., Janshoff, A., Ulrich, W. P., Sieber, M., and Galla, H. J., Impedance analysis of supported lipid bilayer membranes: a scrutiny of different preparation techniques, Biochim. Biophys. Acta 1279 (1996) 169.

[175] Yaroslavov, A. A. et al., Effect of polylysine on transformations and permeability of negative vesicular membranes, Biochimica et Biophysica Acta (BBA) - Biomembranes 1611 (2003) 44.

[176] Russel, W., Saville, D., and Schowalter, W., Colloidal Dispersions, Cambridge, University Press, 1989.

[177] Deserno, M., Elastic deformation of a fluid membrane upon colloid binding, Phys. Rev. E 69 (2004) 031903.

[178] Lee, C.-H., Lin, W.-C., and Wang, J., All-optical measurements of the bending rigidity of lipid-vesicle membranes across structural phase transitions, Phys. Rev. E 64 (2001) 020901.

[179] Kucerka, N., Tristram-Nagle, S., and Nagle, J. F., Structure of Fully Hydrated Fluid Phase Lipid Bilayers with Monounsaturated Chains, J. Membrane Biol. 208 (2005) 193.

[180] Morris, C. E. and Homann, U., Cell surface area regulation and membrane tension, J. Membr. Biol. 179 (2001) 79.

[181] Pécréaux, J., Döbereiner, H. G., Prost, J., Joanny, J., and Bassereau, P., Refined contour analysis of giant unilamellar vesicles, The European Physical Journal E 13 (2004).

[182] Needham, D. and Haydon, D. A., Tensions and free energies of formation of solventless lipid bilayers, Biophysical Journal 41 (1983) 251.

[183] Tien, H. T., Bilayer Lipid Membranes (BLM). Theory and Practice, Marcel Dekker, Inc. New York. 40, 1974.

[184] Tribet, C. and Vial, F., Flexible macromolecules attached to lipid bilayers: impact on fluidity, curvature, permeability and stability of the membranes, Soft Matter 4 (2008) 68. 


\section{Acknowledgements}

It is my pleasure to express my sincere gratitude to many people who helped to make this thesis successful:

Firstly, I with express my thanks to Prof. Dr. Tim Salditt, who made this work possible by giving me the opportunity to pursue a $\mathrm{PhD}$ thesis at the Institute für Röntgenphysik. I thank him for the freedom and support he gave me in this project and for sharing his profound knowledge of $\mathrm{X}$-ray techniques.

I also wish to thank all the co-autors of the publications related to this thesis. Many thanks to belong to Klaus Giewekemayer, Genoveva Mitrea, Christian Peth, Jürgen Thieme, Klaus Mann for fruitful discussions and effective collaborations.

I would like to thank Isabel Pastoriza Santos, Daniela Weiskopf, Ruth Szilluweit, Tanja Ducic, Frank Barkusky, André Beerlink, Jean Susini, Till Hartmut Metzger, Peter Nieschalk, Thorsten Gronemann and Jochen Herbst for their experimental help and support.

I also thank Peter, Tanja, Simon, Sajal, Sophie-Charlotte and Sebastian for proof-reading parts of the manuscript.

I gratefully acknowledge financial support by European Network of Excellence on Soft Composite Materials (SOFTCOMP) and SFB755 "Nanoscale Photonic Imaging".

Last but not the least, I would like to thank my husband Peter and my parents for their support, patience, and love all through these years. 



\section{List of publications}

E. Nováková, G. Mitrea, C. Peth, J. Thieme, K. Mann, and T. Salditt, Solid supported multicomponent lipid membranes studied by x-ray spectromicroscopy, Biointerphases 3(2) (2008) FB44-FB54.

E. Nováková, K. Giewekemeyer, and T. Salditt, Structure of two-component lipid membranes on solid support: An x-ray reflectivity study, Physical Review E 74 (2006) 051911.

Y. Hwu, W. L. Tsai, H. M. Chang, H. I. Yeh, P. C. Hsu, Y. C. Yang, Y. T. Su, H. L. Tsai, G. M. Chow, P. C. Ho, S. C. Li, H. O. Moser, P. Yang, S. K. Seol, C. C. Kim, J. H. Je, E. Stefanekova, A. Groso, and G. Margaritondo, Imaging Cells and Tissues with Refractive Index Radiology, Biophys. J. 87 (2004) 4180-4187. 



\section{Lebenslauf}

Eva Nováková, geb. Štefáneková

Geboren am 1. September 1980 in Ružomberok, Slowakei

Nationalität: Slowakisch

\section{Ausbildung}

02/2005 - 07/2008 Röntgenphysik/Biophysik (Promotionsstudium)

Georg-August Universität, Göttingen, Deutschland

Leiter: Prof. Dr. Tim Salditt

Thema: $\quad$ Structure of charged two-component lipid membranes and their interaction with colloids studied by different $X$-ray and microscopy techniques

1998 - 2003 Physik/Biomedizinische Physik (Mgr./MSc. äquivalent)

Comenius University, Bratislava, Slowakei

Leiter: Doc. RNDr. Ján Urban, CSc.

Thema: $\quad$ Computer modelling of interactions of $P_{24}$ peptid with selected membranes

1994 - 1998 Abitur, Gymnasium in Ružomberok, Slowakei 